\title{
Dual Solution/Solid-State Emissive Excited-State Intramolecular Proton Transfer (ESIPT) Dyes: A Combined Experimental and Theoretical Approach
}

Thibault Pariat, ${ }^{\dagger \mid}$ Timothée Stoerkler, ${ }^{\dagger \mid}$ Clément Diguet, ${ }^{\ddagger}$ Adèle D. Laurent, ${ }^{*}+$ Denis Jacquemin, ${ }^{*}+$ Gilles Ulrich ${ }^{* \dagger}$ and Julien Massue ${ }^{* \dagger}$

" These authors contributed equally to the work

† Institut de Chimie et Procédés pour l'Energie, l'Environnement et la Santé (ICPEES), Equipe Chimie Organique pour la Biologie, les Matériaux et l'Optique (COMBO), UMR CNRS 7515, Ecole Européenne de Chimie, Polymères et Matériaux (ECPM), Université de Strasbourg, 25 Rue Becquerel, 67087 Strasbourg Cedex 02, France

\$aboratoire CEISAM UMR UN-CNRS 6230, Université de Nantes, Nantes F-44000, France Email: Adele.Laurent@,univ-nantes.fr, Denis.Jacquemin@univ-nantes.fr, gulrich@unistra.fr, massue@,unistra.fr 


\section{Table of contents}

S1 Materials and Methods, p.S3

S2 ${ }^{1} \mathrm{H}$ and ${ }^{13} \mathrm{C}$ NMR spectra of HBO dyes 1-16, p.S5

S3 Spectroscopic data, p.S22

S4 Additional theoretical data, p.S61

S5 References, p.S137 


\section{S1. Materials and methods: Experiment}

All commercially available chemicals were received from Sigma Aldrich or Fluorochem. Tetrahydrofuran (THF) was distilled over sodium/benzophenone under argon. Dichloromethane was distilled over $\mathrm{P}_{2} \mathrm{O}_{5}$ under argon. Triethylamine $\left(\mathrm{Et}_{3} \mathrm{~N}\right)$ was distilled over potassium hydroxide under argon. Thin layer chromatographies (TLC) were performed on silica gel coated with a fluorescent indicator. Purifications by column chromatography were conducted using 40-63 $\mu \mathrm{m}$ silica gel.

${ }^{1} \mathrm{H}$ NMR $(500 \mathrm{MHz})$ and ${ }^{13} \mathrm{C}$ NMR $(126 \mathrm{MHz})$ spectra were recorded on a Bruker Advance spectrometer with deuterated solvents. Mass spectrometry analysis was performed by direct sample injection on a electrospray ionization time-of-flight (ESI-TOF) micrOTOF II mass spectrometer. Absorption spectra were recorded using a dual-beam grating Schimadzu UV3000 absorption spectrometer with a quartz cuvette of $1 \mathrm{~cm}$ of optical path length. The steadystate fluorescence emission and excitation spectra were recorded by using a Horiba S2 Jobin Yvon Fluoromax 4. All fluorescence and excitation spectra were corrected. Solvents for spectroscopy were spectroscopic grade.

The fluorescence quantum yields $\left(\Phi_{\text {exp }}\right)$ were measured in diluted solution with an absorption value below 0.1 at the excitation wavelength using the following equation:

$$
\Phi_{\exp }=\Phi_{r e f} \frac{\mathrm{I}}{\mathrm{I}_{r e f}} \frac{\mathrm{OD}_{r e f}}{\mathrm{OD}} \frac{\eta^{2}}{\eta^{2}{ }_{r e f}} \quad \text { Equation } \mathrm{S}_{1}
$$

I is the integral of the corrected emission spectrum, OD is the optical density at the excitation wavelength, and $\eta$ is the refractive index of the medium. The reference system used was Rhodamine $6 \mathrm{G}, \Phi=88 \%$ in ethanol $\left(\lambda_{\mathrm{exc}}=488 \mathrm{~nm}\right)$.

In the case of dual $\mathrm{E}^{*} / \mathrm{K}^{*}$ emission, global quantum yields are provided, i.e. taking the area of both bands into account in the calculation.

For solid-state measurements, $\mathrm{KBr}(200 \mathrm{mg})$ and a given $\mathrm{HBO}$ dye $(2 \mathrm{mg}$, i.e. $1 \% \mathrm{wt})$ are ground together in a mortar. A pellet is subsequently made and the photophysical data recorded (absolute quantum yield using an integration sphere and emission/excitation spectra).

Luminescence lifetimes were measured on a Horiba Scientific TCSPC system equipped with a nanoLED 370. Lifetimes were deconvoluted with FS-900 software using a light-scattering 
solution $\left(\right.$ LUDOX) for instrument response. The excitation source was a laser diode $\left(\lambda_{\text {exc }}=\right.$ $320 \mathrm{~nm})$.

\section{Materials and methods: Theory}

To model ESIPT we have relied on Time-Dependent Density Functional Theory (TD-DFT). More precisely, we have optimized and computed the vibrational frequencies of the enol, keto, and proton transition state in their first excited state, the latter showing an imaginary frequency corresponding to the ESIPT phenomena. This allowed computing the relative free energies of the three species, and hence to determine if a driving force and a small barrier allow for ESIPT to take place. These calculations were performed with the M06-2X functional $^{1}$ and the $6-31 \mathrm{G}(\mathrm{d})$ atomic basis set, including solvent effects (toluene or dicholoromethane) using the standard linear-response (LR) Polarizable Continuum Model $(\mathrm{PCM}){ }^{2}$ These calculations, like all TD-DFT calculations herein were performed with Gaussian $16 .{ }^{3}$ For the fluorescence wavelengths, we used a more refined composite protocols, inspired by some of our previous works. ${ }^{4}$ More in details, considering the above geometries, we first computed the transition energies with TD-M06-2X/6-311+G(2d,p) in both gas and solution, using the LR+cLR model for the latter, ${ }^{5}$ that accounts for both linear-response and state-specific solvent effects. Secondly we computed the transition energies in gas-phase at the second-order CC level (CC2), ${ }^{6}$ with the aug-cc-pVTZ atomic basis set. These latter calculations were performed with the Turbomole package, ${ }^{7}$ applying the RI approximation. It was then possible to obtain corrected transition energies, following a simple protocol described elsewhere. ${ }^{4}$ For both Gaussian and Turbomole calculations, we used at least the default convergence thresholds, generally improving them. 


\section{S2 ${ }^{1} \mathrm{H}$ and ${ }^{13} \mathrm{C}$ NMR spectra}

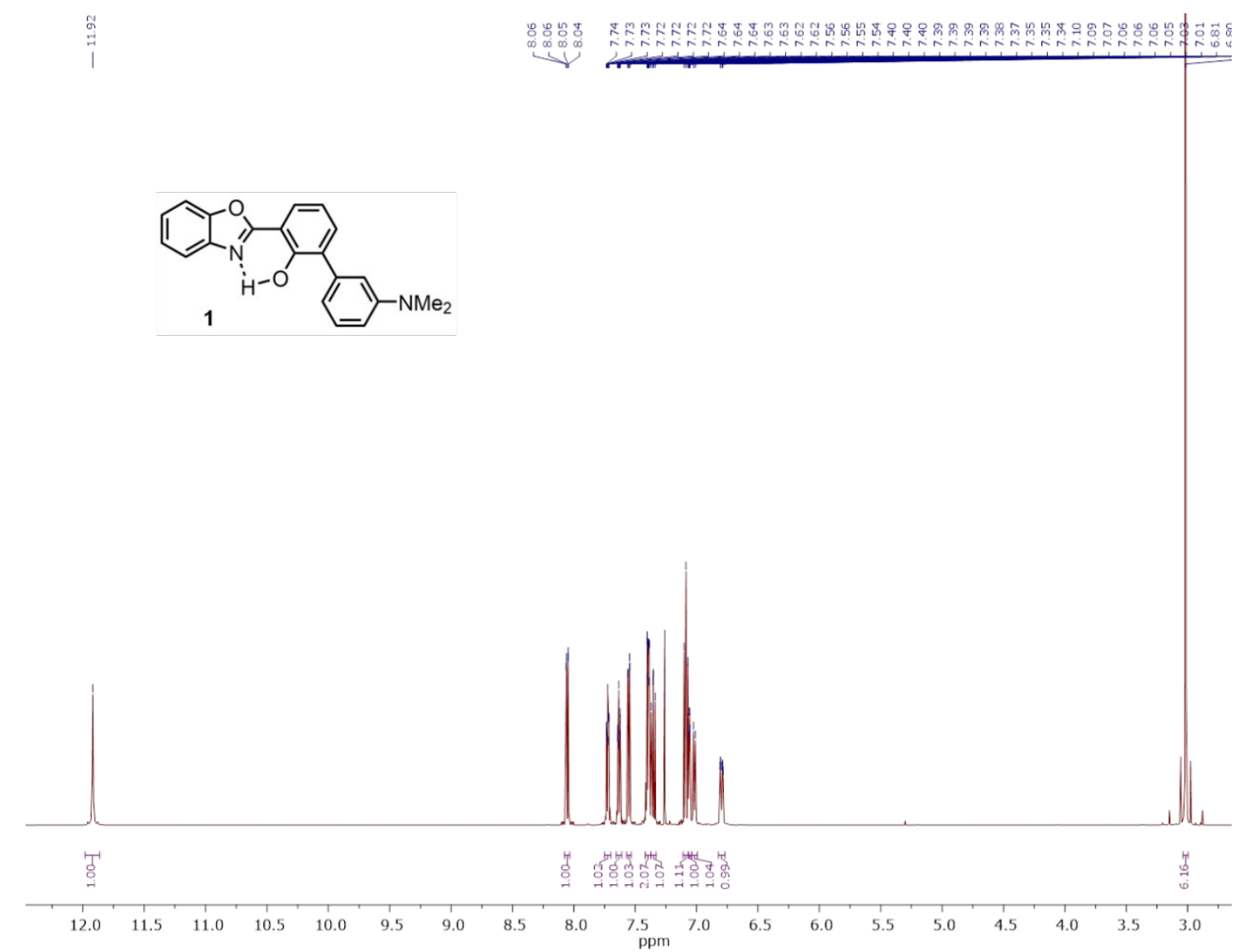

Figure S1. ${ }^{1} \mathrm{H} \mathrm{NMR}\left(\mathrm{CDCl}_{3}, 500 \mathrm{MHz}\right)$, spectrum of $\mathrm{HBO} 1$
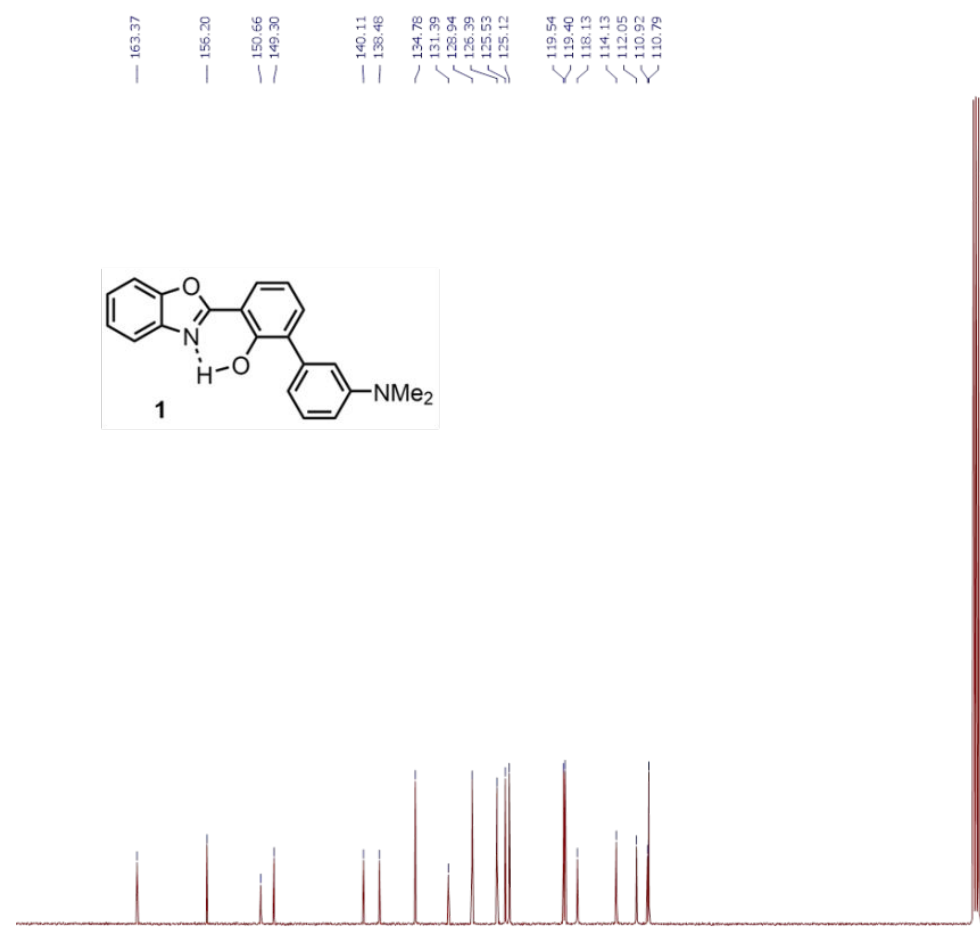


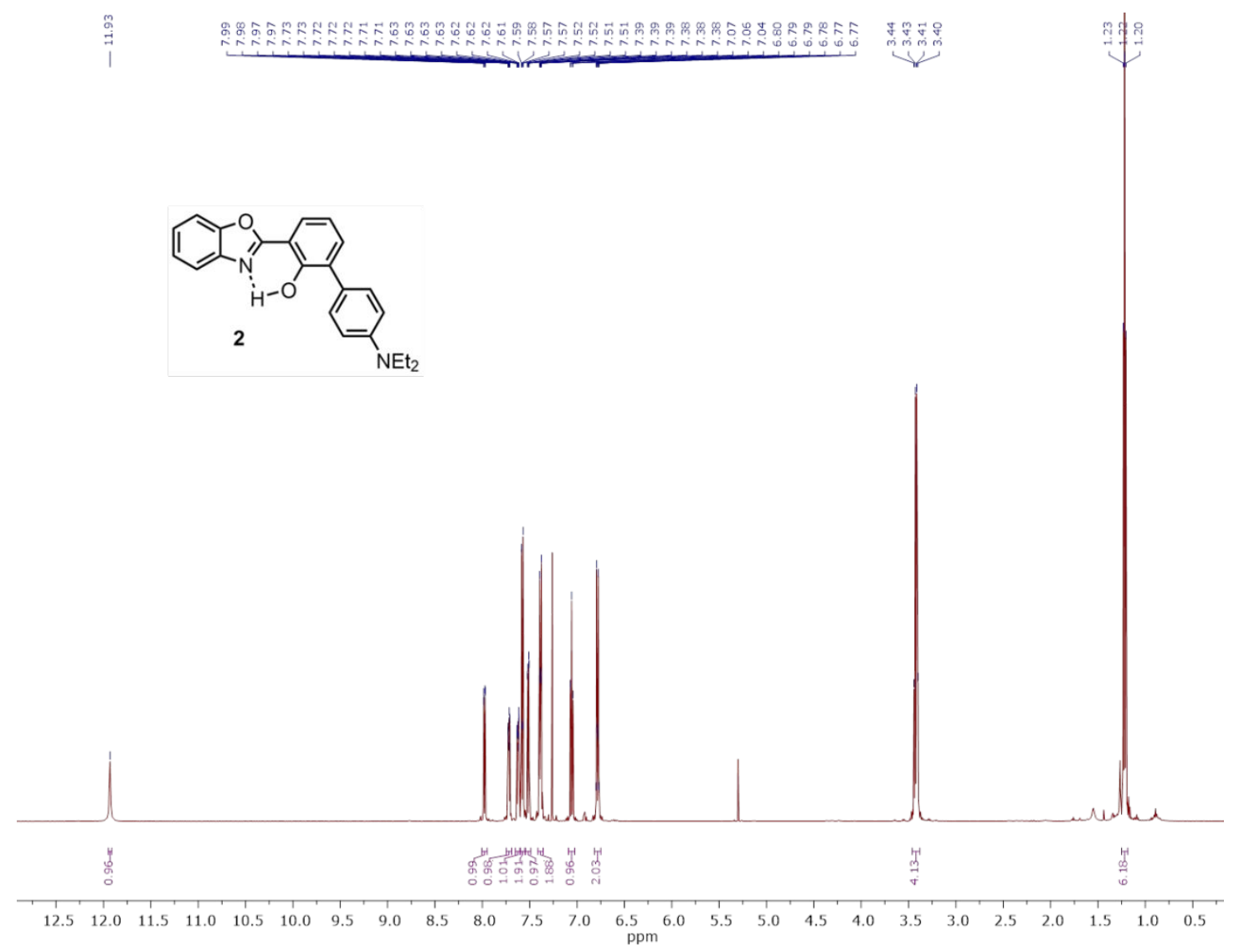

Figure S3. ${ }^{1} \mathrm{H} \mathrm{NMR}\left(\mathrm{CDCl}_{3}, 500 \mathrm{MHz}\right)$, spectrum of $\mathrm{HBO} 2$
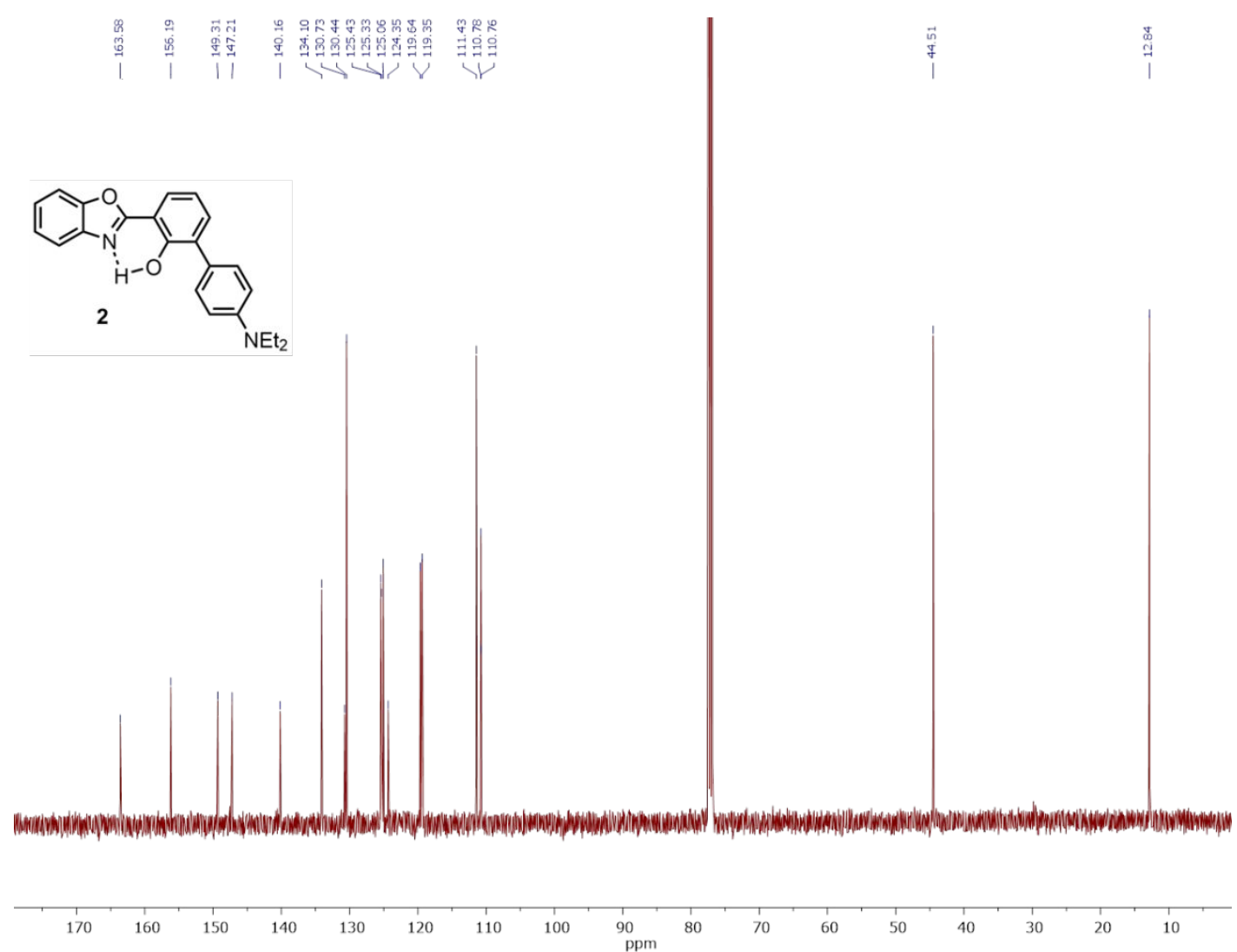

Figure S4. ${ }^{13} \mathrm{C} \mathrm{NMR}\left(\mathrm{CDCl}_{3}, 126 \mathrm{MHz}\right)$, spectrum of $\mathrm{HBO} 2$ 

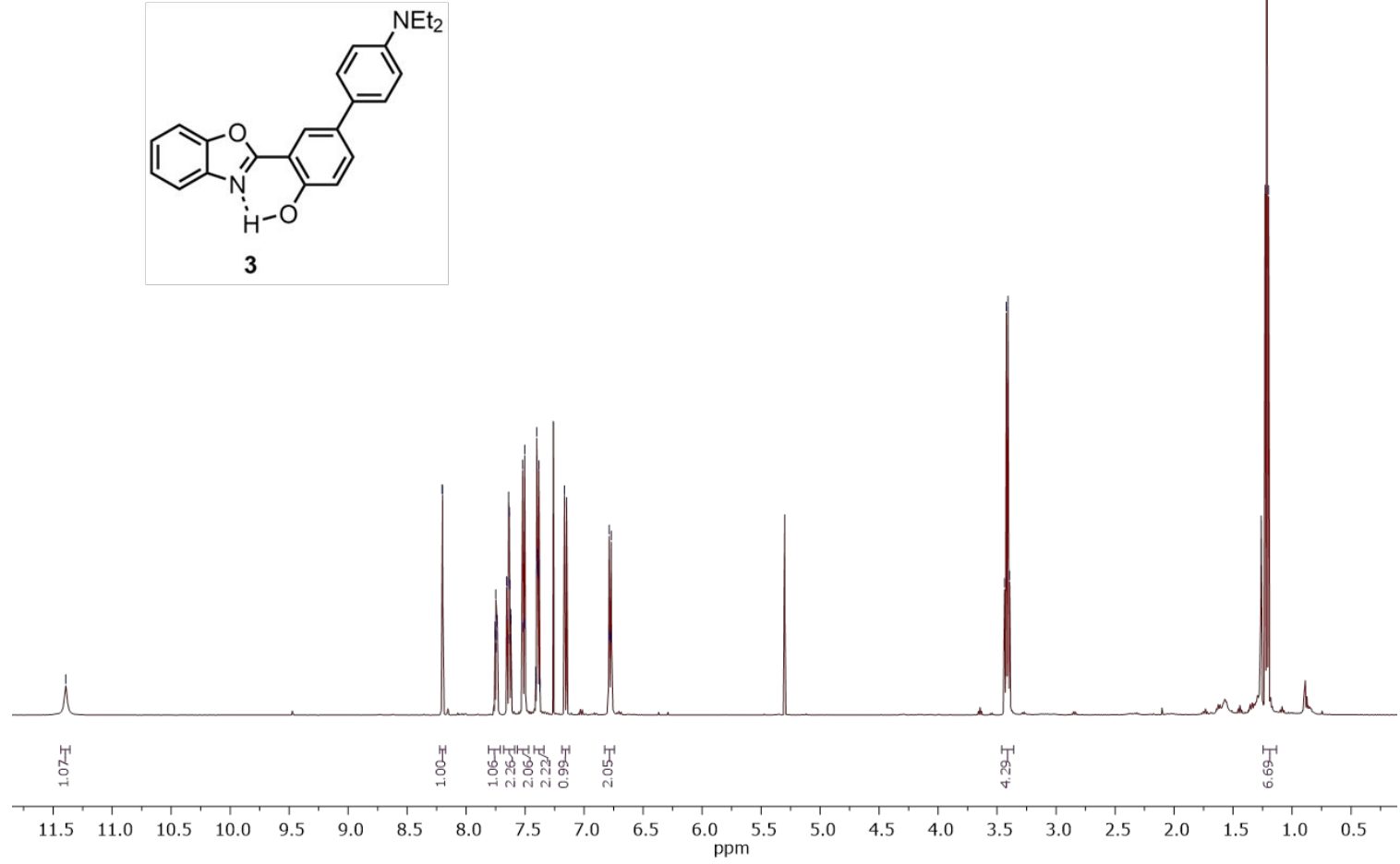

Figure S5. ${ }^{1} \mathrm{H} \mathrm{NMR}\left(\mathrm{CDCl}_{3}, 500 \mathrm{MHz}\right)$, spectrum of $\mathrm{HBO} 3$
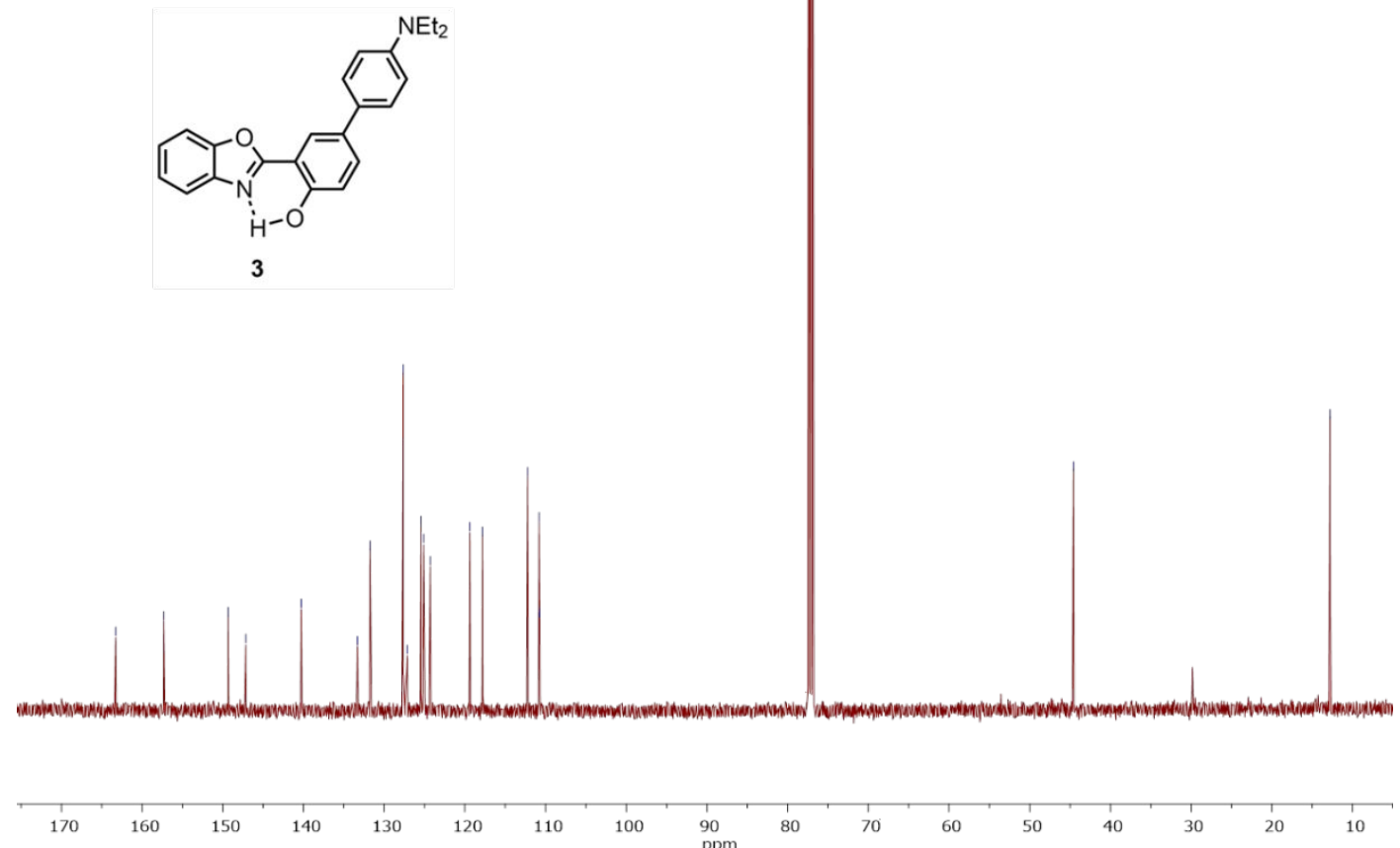

Figure S6. ${ }^{13} \mathrm{C} \mathrm{NMR}\left(\mathrm{CDCl}_{3}, 126 \mathrm{MHz}\right)$, spectrum of $\mathrm{HBO} 3$ 


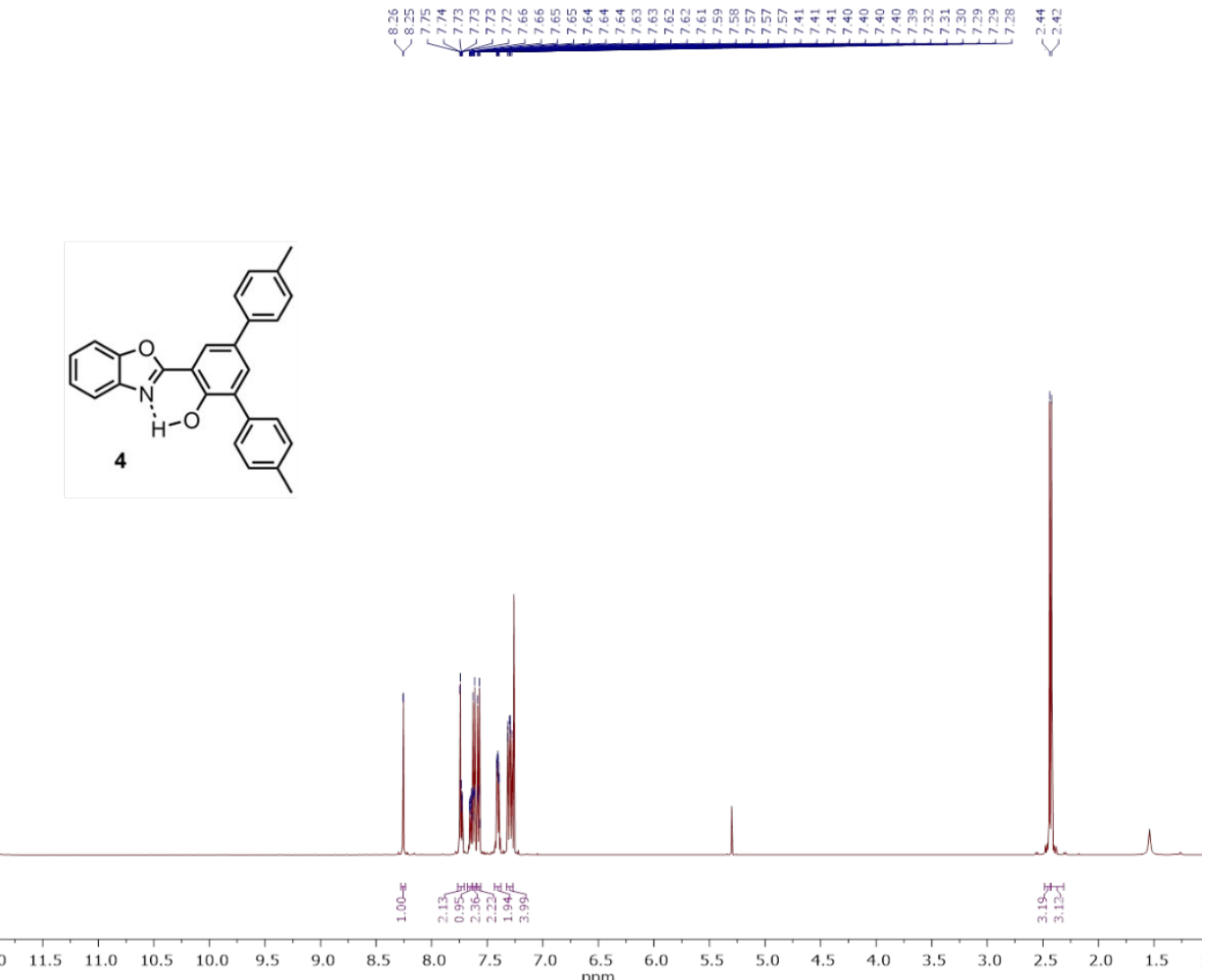

Figure S7. ${ }^{1} \mathrm{H} \mathrm{NMR}\left(\mathrm{CDCl}_{3}, 500 \mathrm{MHz}\right)$, spectrum of $\mathrm{HBO} 4$

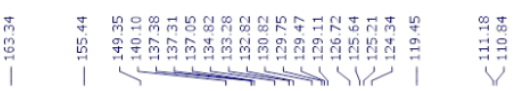
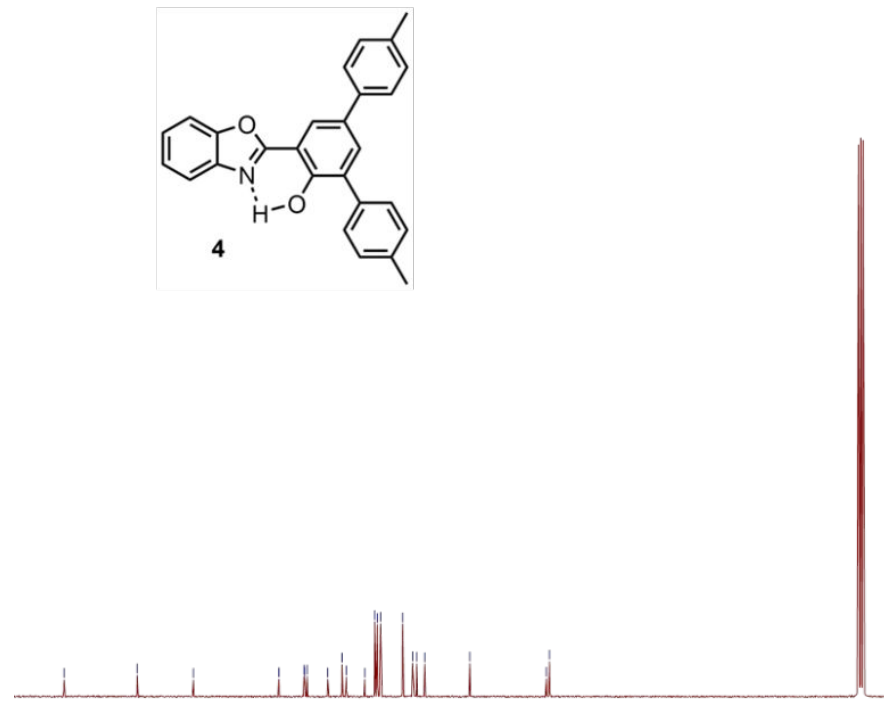

\begin{tabular}{|c|c|c|c|c|c|c|c|c|c|c|c|c|c|}
\hline 160 & 150 & 140 & 130 & 120 & 110 & 100 & 90 & 80 & 70 & 60 & 50 & 40 & 30 \\
\hline
\end{tabular}

Figure S8. ${ }^{13} \mathrm{C} \mathrm{NMR}\left(\mathrm{CDCl}_{3}, 126 \mathrm{MHz}\right)$, spectrum of $\mathrm{HBO} 4$ 

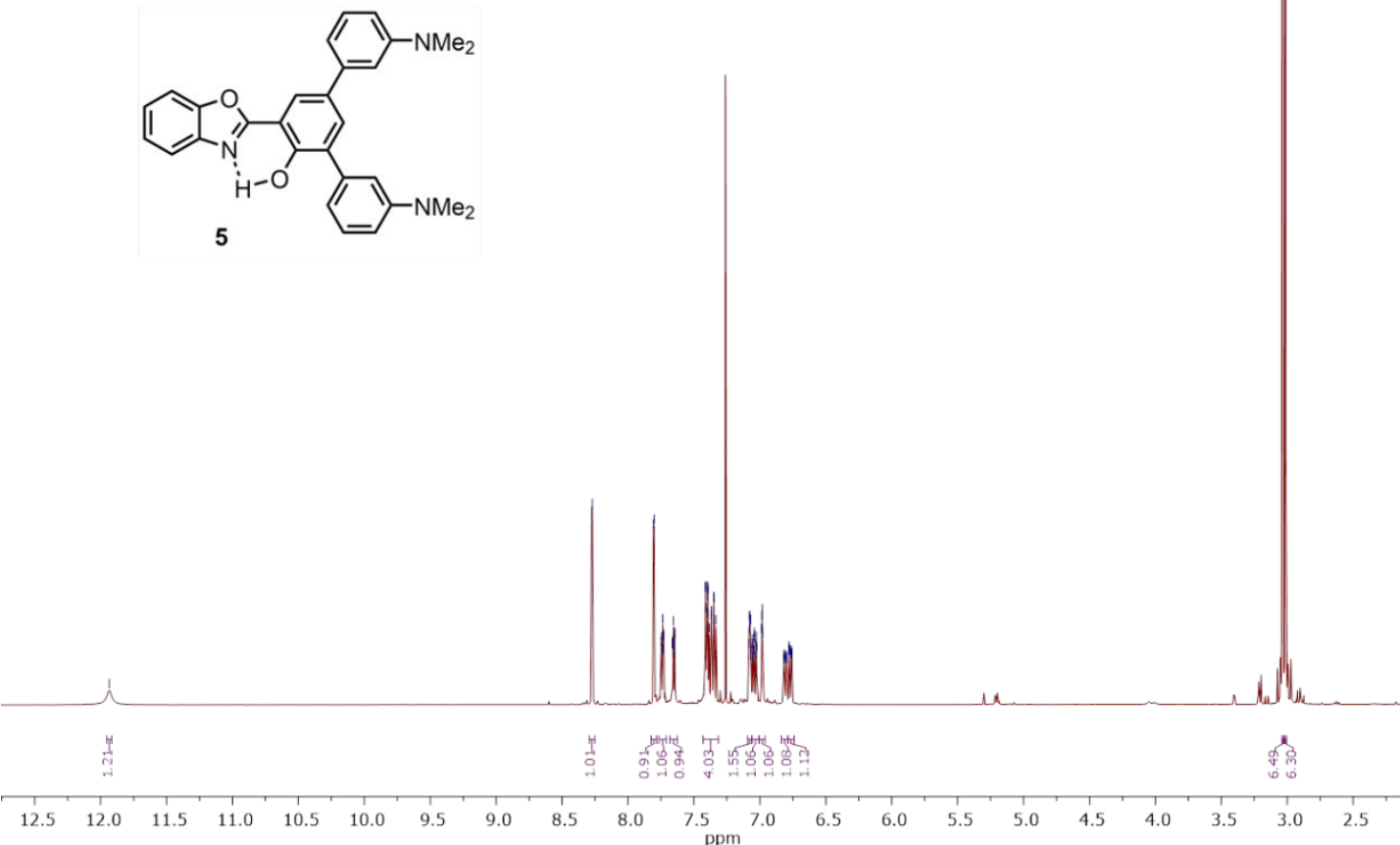

Figure S9. ${ }^{1} \mathrm{H} \mathrm{NMR}\left(\mathrm{CDCl}_{3}, 500 \mathrm{MHz}\right)$, spectrum of $\mathrm{HBO} 5$
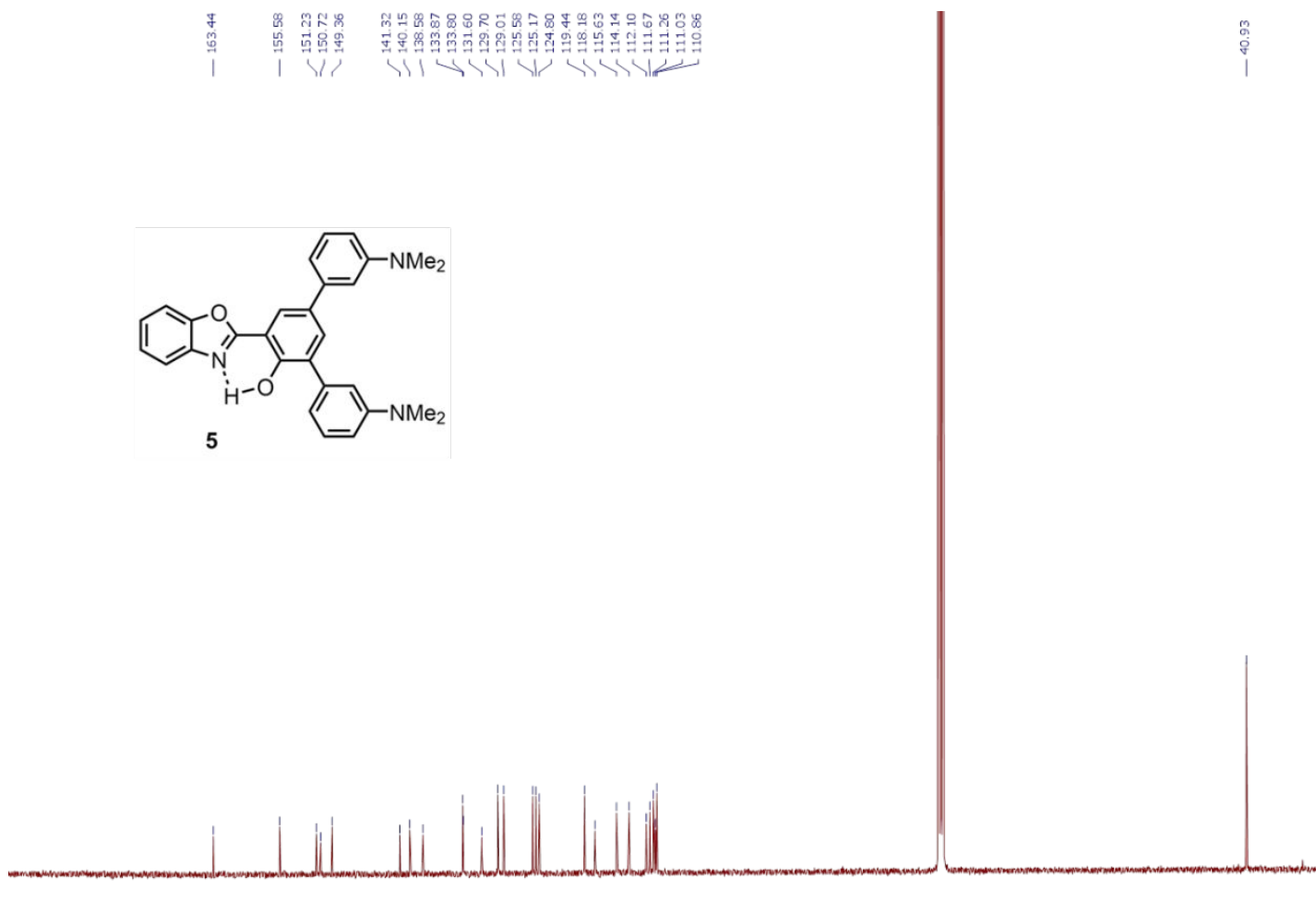

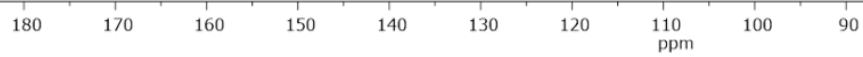

Figure S10. ${ }^{13} \mathrm{C} \mathrm{NMR}\left(\mathrm{CDCl}_{3}, 126 \mathrm{MHz}\right)$, spectrum of $\mathrm{HBO} 5$ 


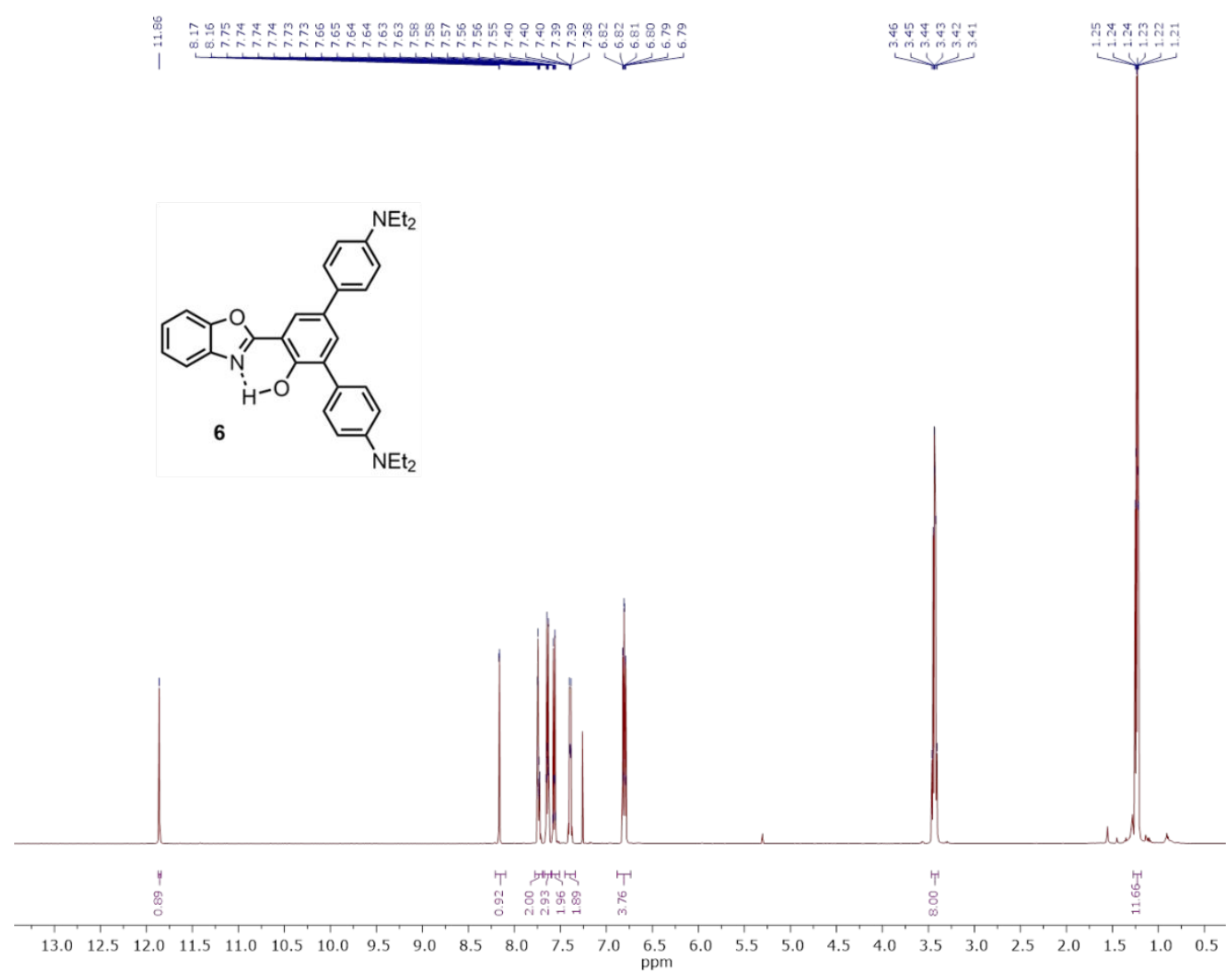

Figure S11. ${ }^{1} \mathrm{H} \mathrm{NMR}\left(\mathrm{CDCl}_{3}, 500 \mathrm{MHz}\right)$, spectrum of $\mathrm{HBO} 6$

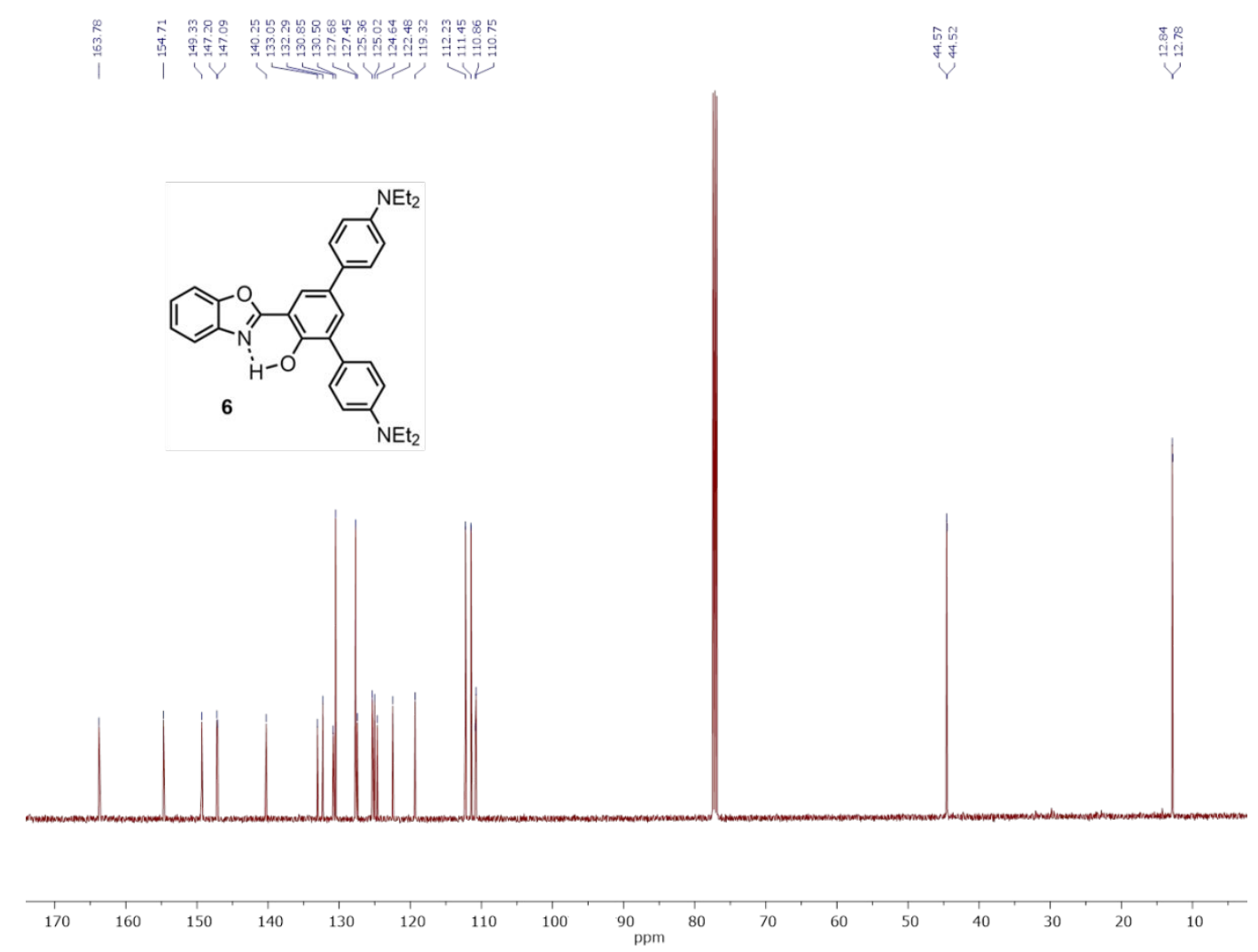


I

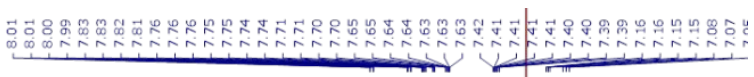
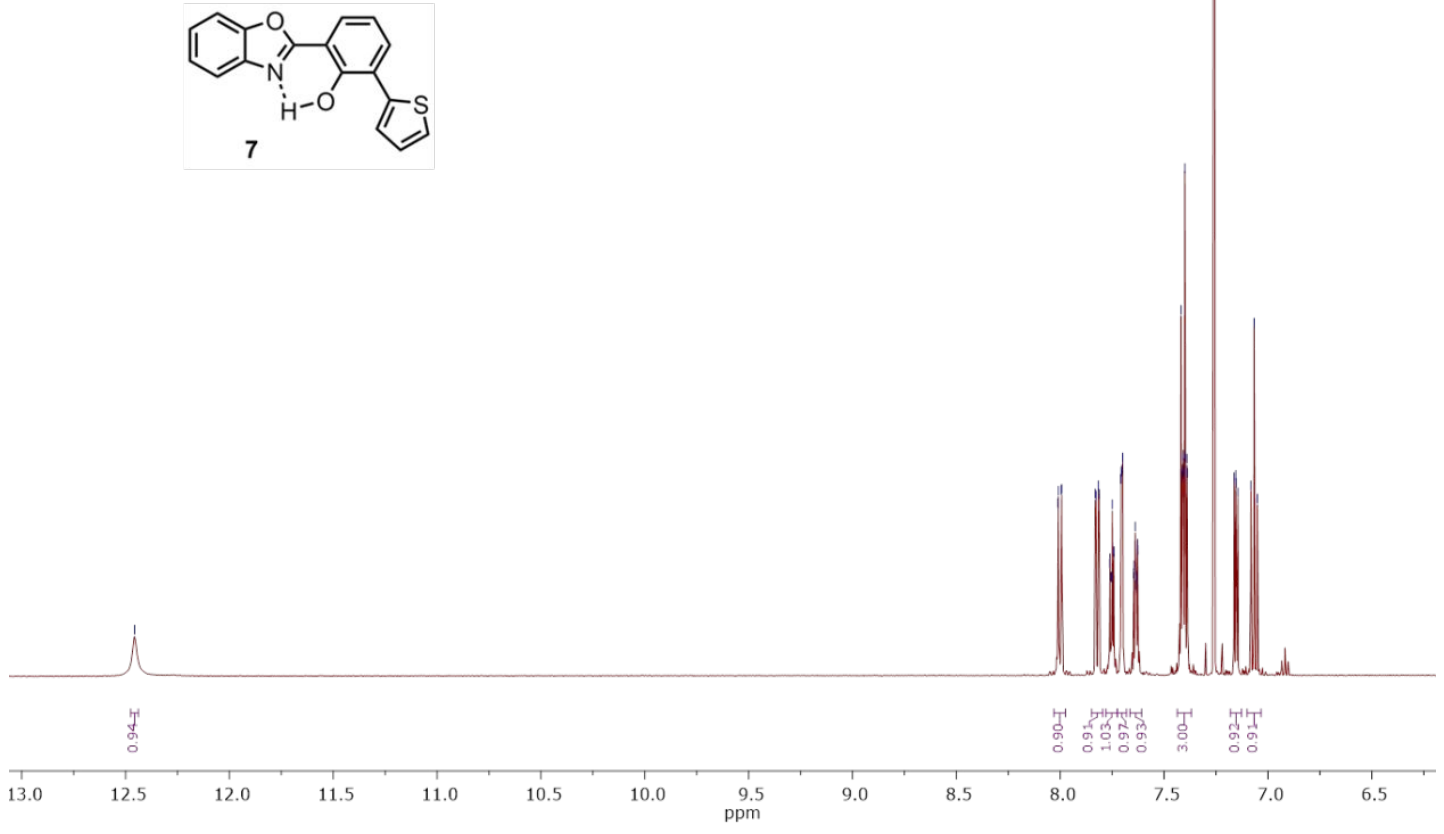

Figure S13. ${ }^{1} \mathrm{H} \mathrm{NMR}\left(\mathrm{CDCl}_{3}, 500 \mathrm{MHz}\right)$, spectrum of $\mathrm{HBO} 7$

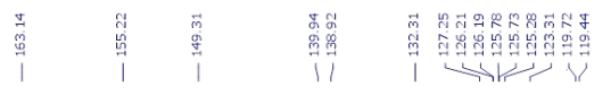

员盟
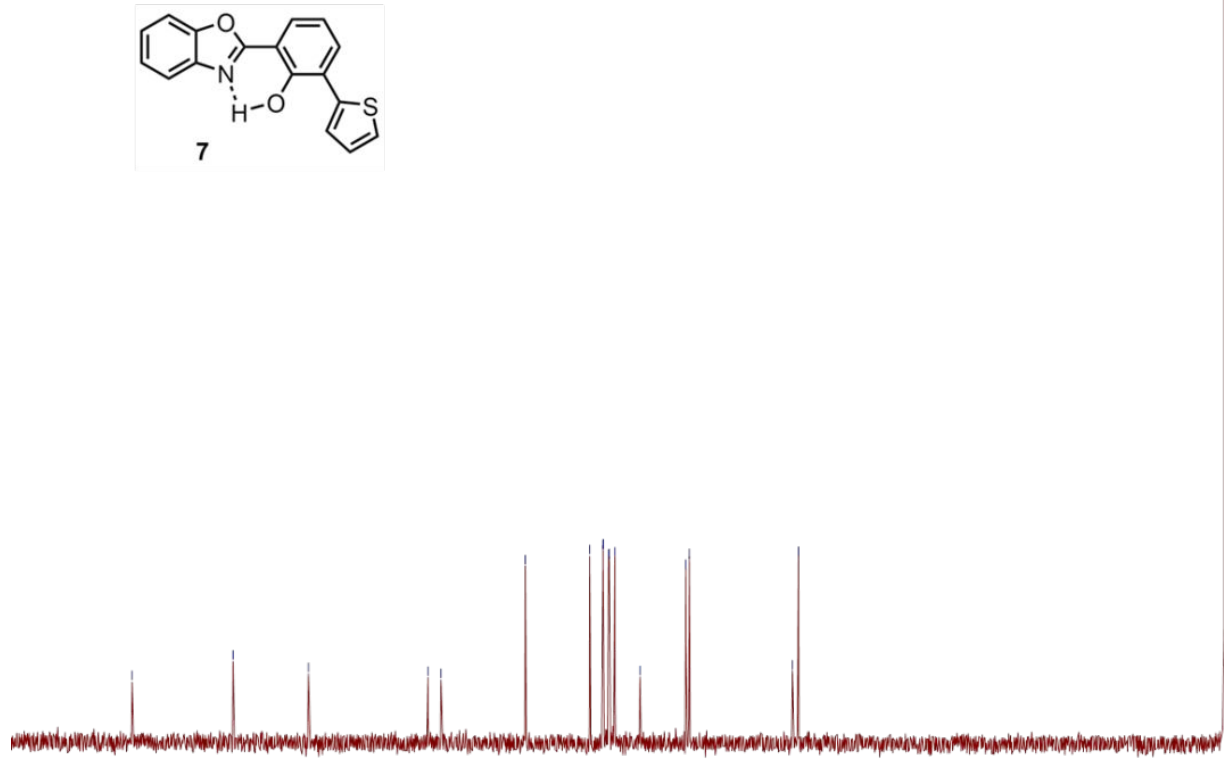

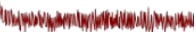

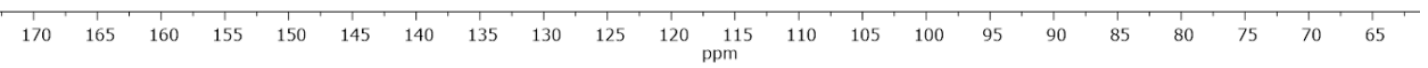

Figure S14. ${ }^{13} \mathrm{C} \mathrm{NMR}\left(\mathrm{CDCl}_{3}, 126 \mathrm{MHz}\right)$, spectrum of $\mathrm{HBO} 7$

$\mathrm{S} 11$ 


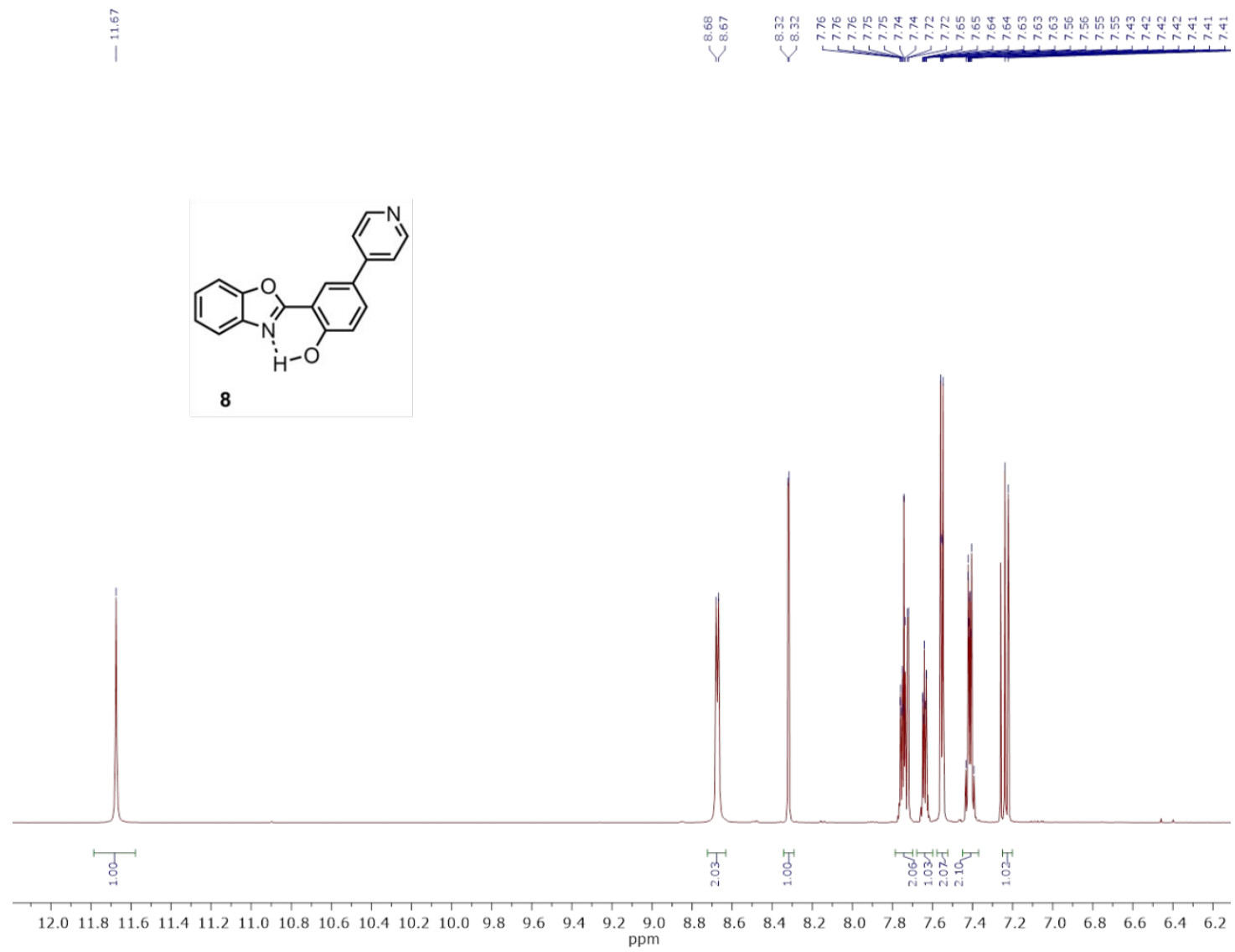

Figure S15. ${ }^{1} \mathrm{H} \mathrm{NMR}\left(\mathrm{CDCl}_{3}, 500 \mathrm{MHz}\right)$, spectrum of $\mathrm{HBO} 8$
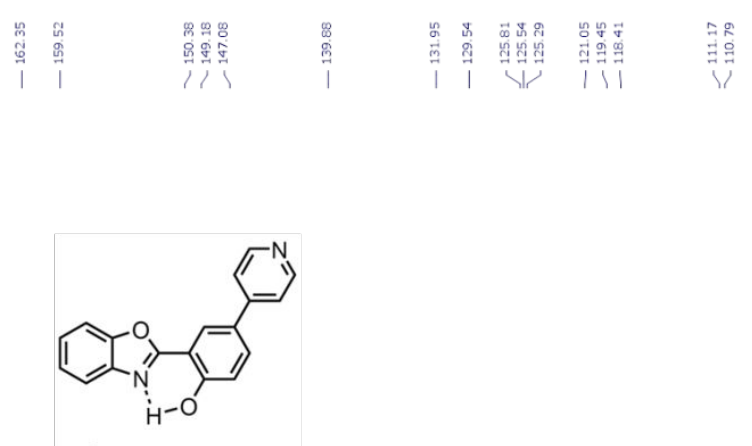

8

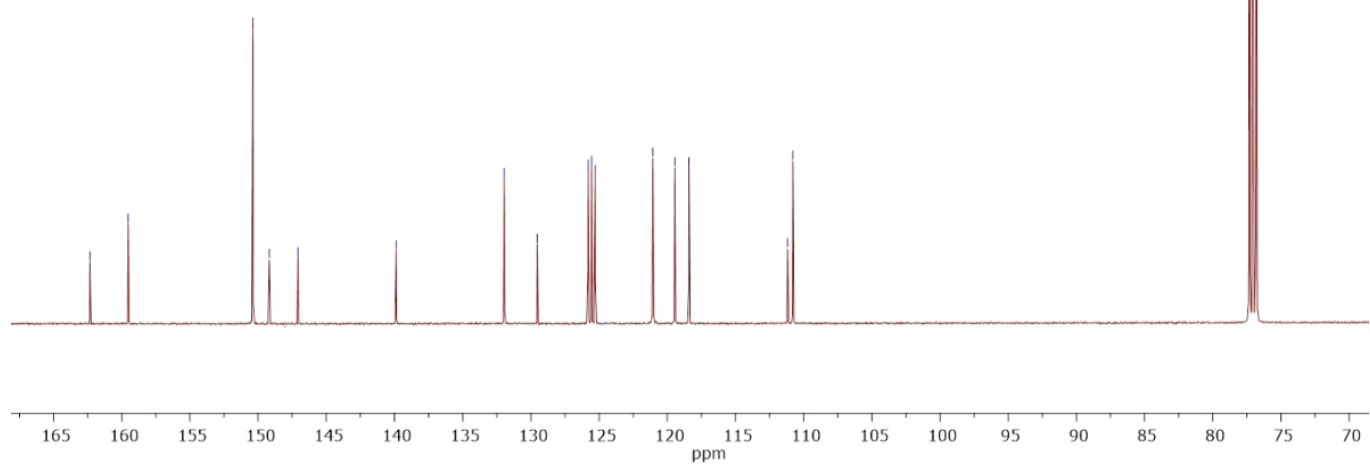

Figure S16. ${ }^{13} \mathrm{C}$ NMR $\left(\mathrm{CDCl}_{3}, 126 \mathrm{MHz}\right)$, spectrum of $\mathrm{HBO} 8$ 


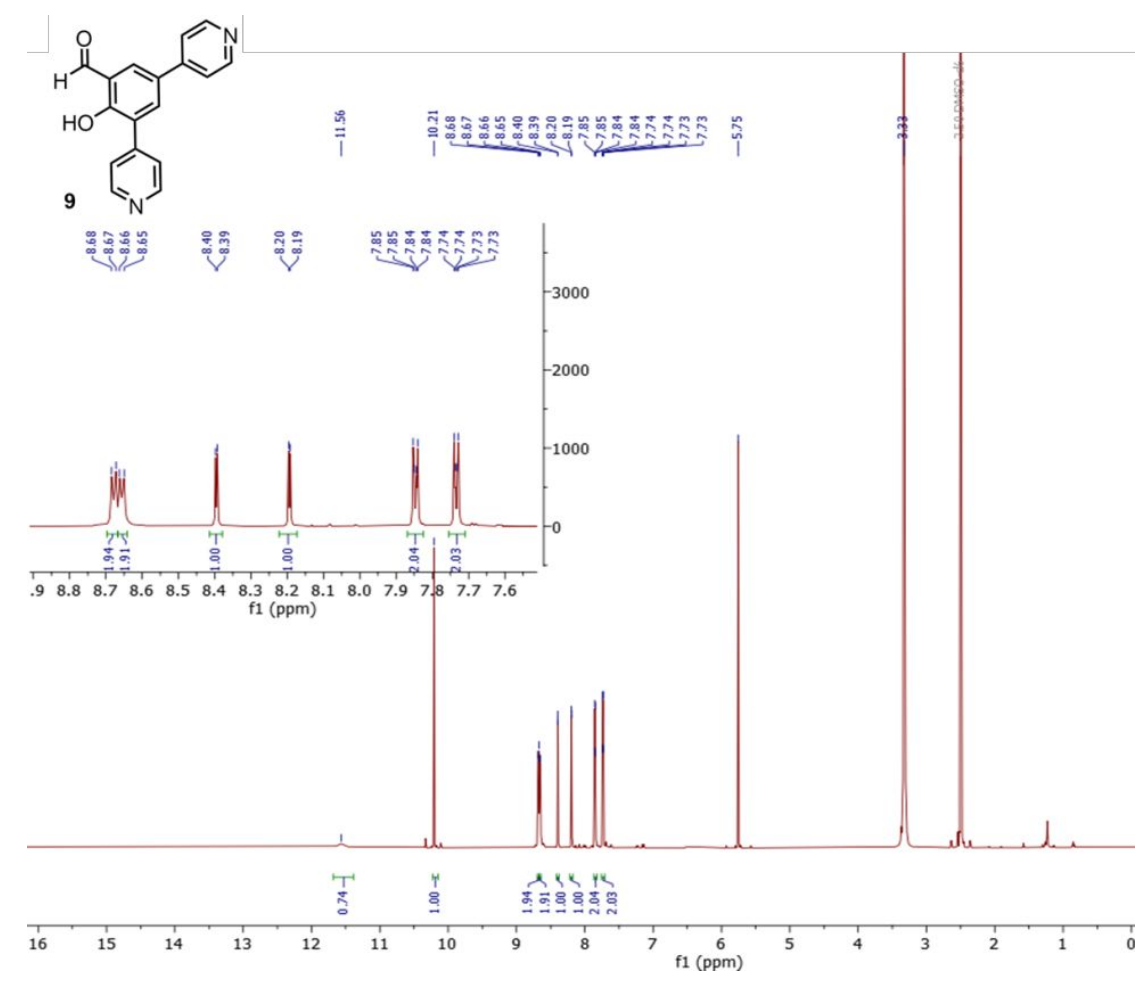

Figure S17. ${ }^{1} \mathrm{H}$ NMR $\left({ }^{6} \mathrm{~d}\right.$-DMSO, $\left.500 \mathrm{MHz}\right)$ spectrum of salicylaldehyde 9

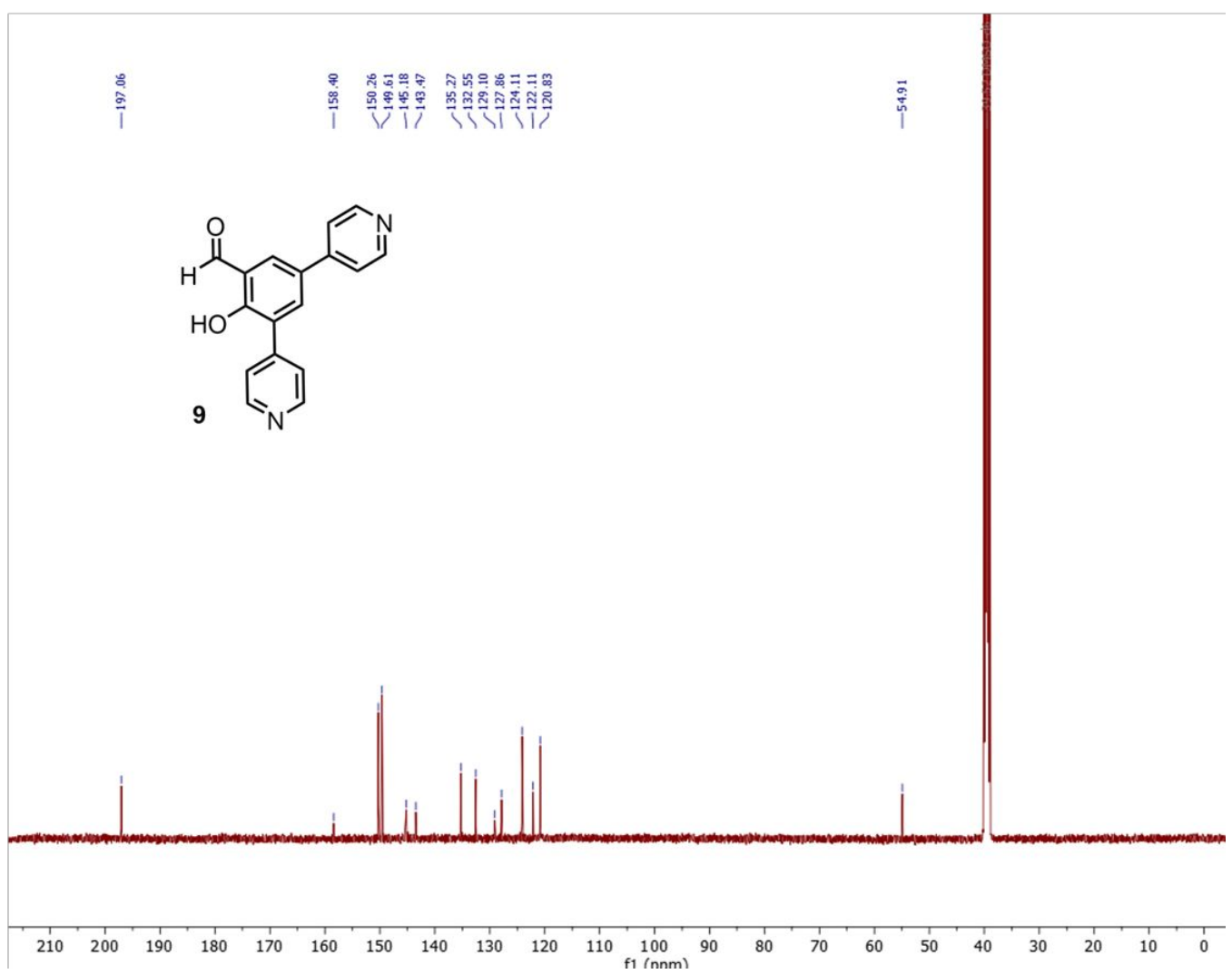

Figure S18. ${ }^{13} \mathrm{C}$ NMR $\left({ }^{6} \mathrm{~d}\right.$-DMSO, $\left.126 \mathrm{MHz}\right)$ spectrum of salicylaldehyde 9 


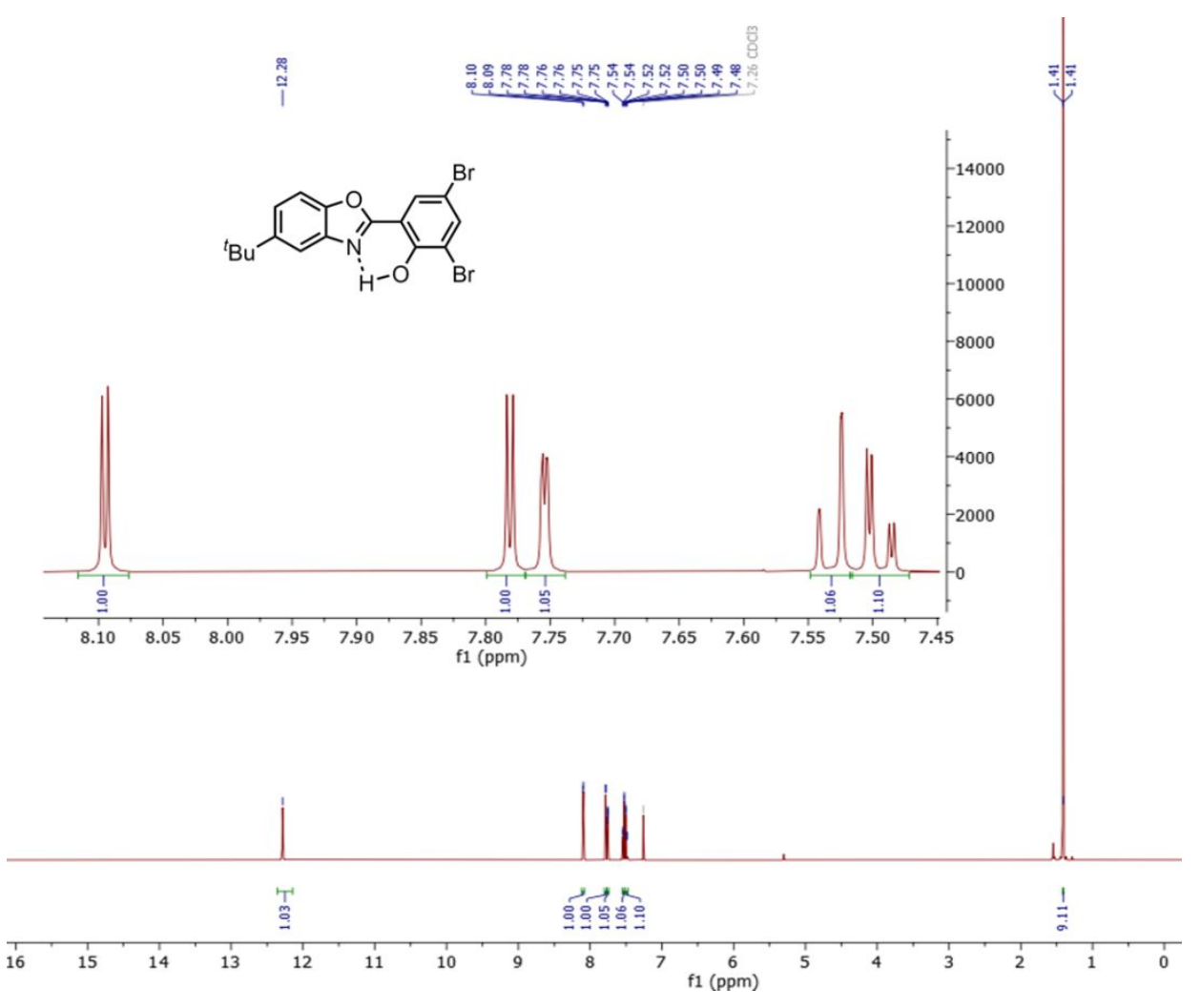

Figure S19. $\left.{ }^{1} \mathrm{H} \mathrm{NMR} \mathrm{(CDCl}{ }_{3}, 500 \mathrm{MHz}\right)$, spectrum of 3,5-bromo-2-(2'-hydroxyphenyl)-5tertiobutylbenzoxazole

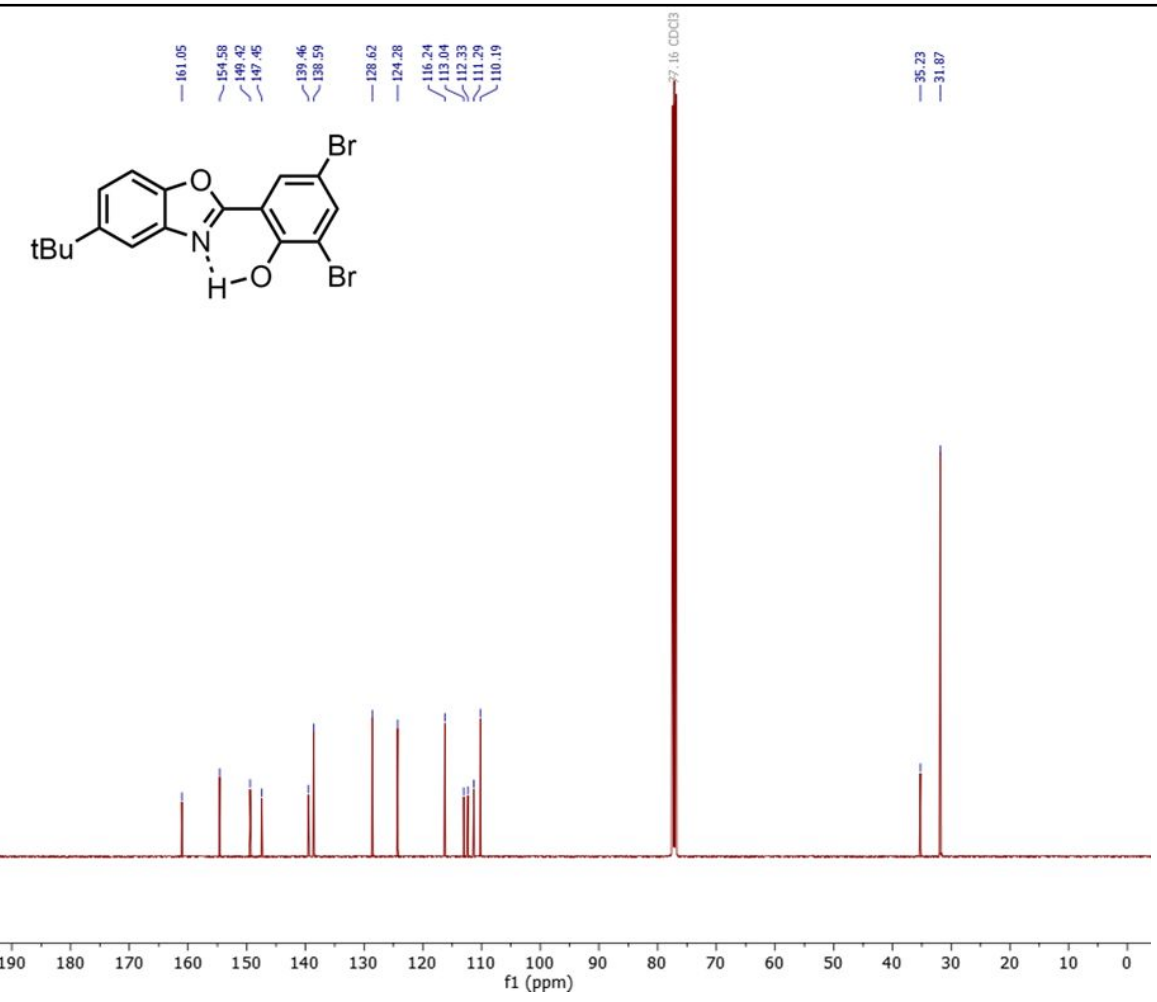

Figure S20. $\left.{ }^{13} \mathrm{C} \mathrm{NMR} \mathrm{(CDCl}, 126 \mathrm{MHz}\right)$, spectrum of 3,5-bromo-2-(2'-hydroxyphenyl)-5tertiobutylbenzoxazole 


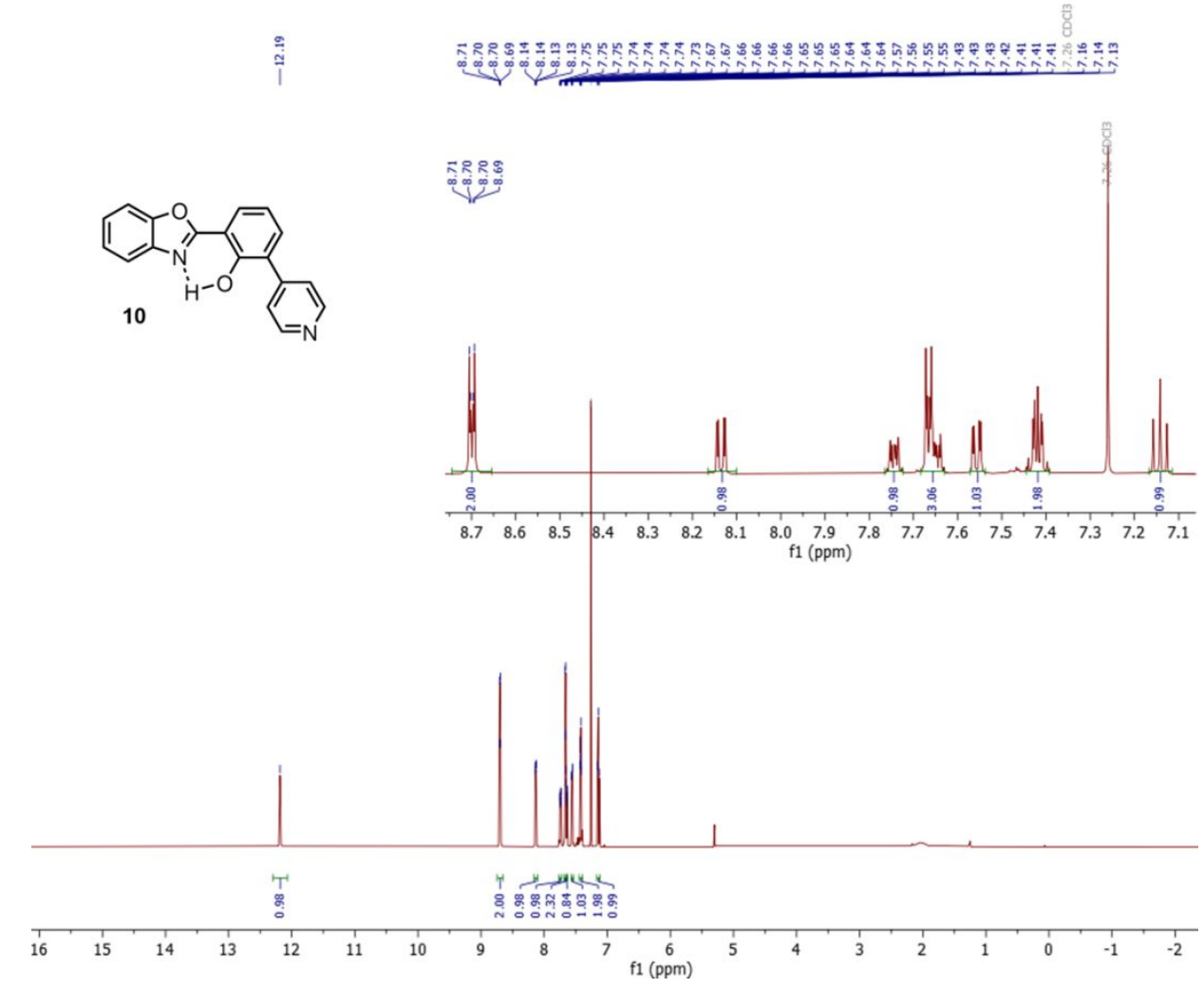

Figure S21. ${ }^{1} \mathrm{H} \mathrm{NMR}\left(\mathrm{CDCl}_{3}, 500 \mathrm{MHz}\right)$, spectrum of $\mathrm{HBO} 10$

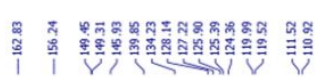
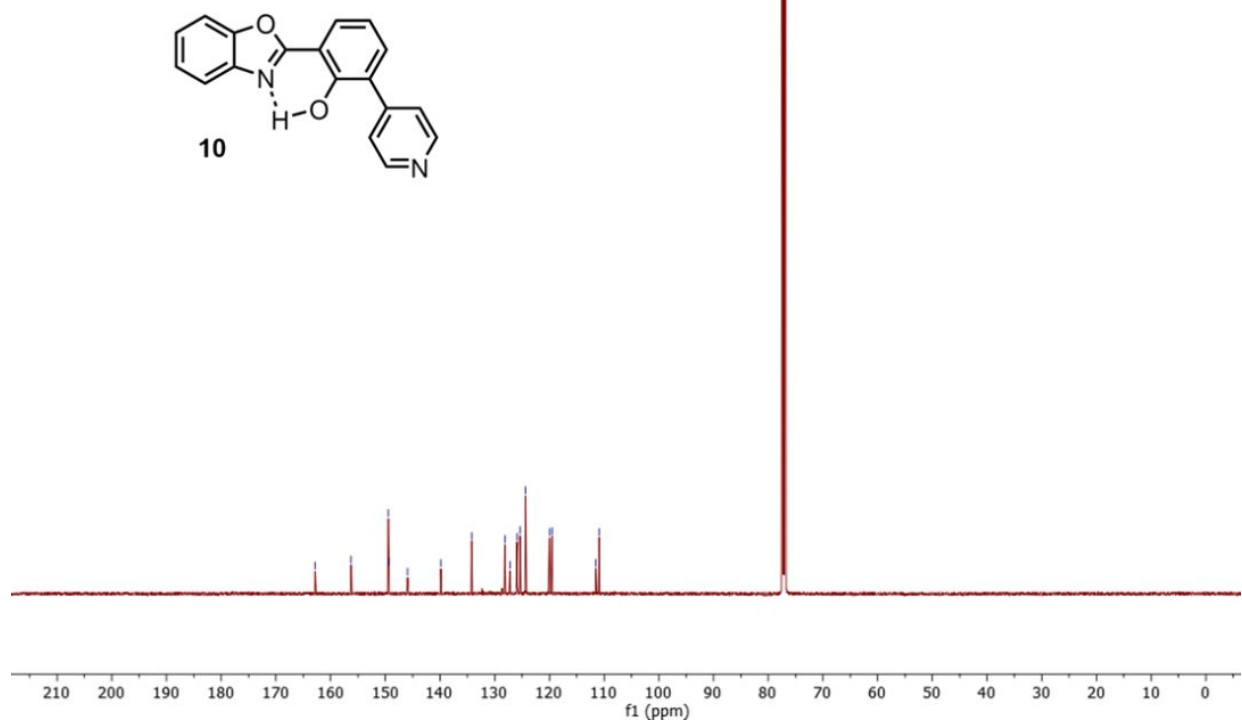

Figure S22. ${ }^{13} \mathrm{C}$ NMR $\left(\mathrm{CDCl}_{3}, 126 \mathrm{MHz}\right)$, spectrum of $\mathrm{HBO} 10$ 

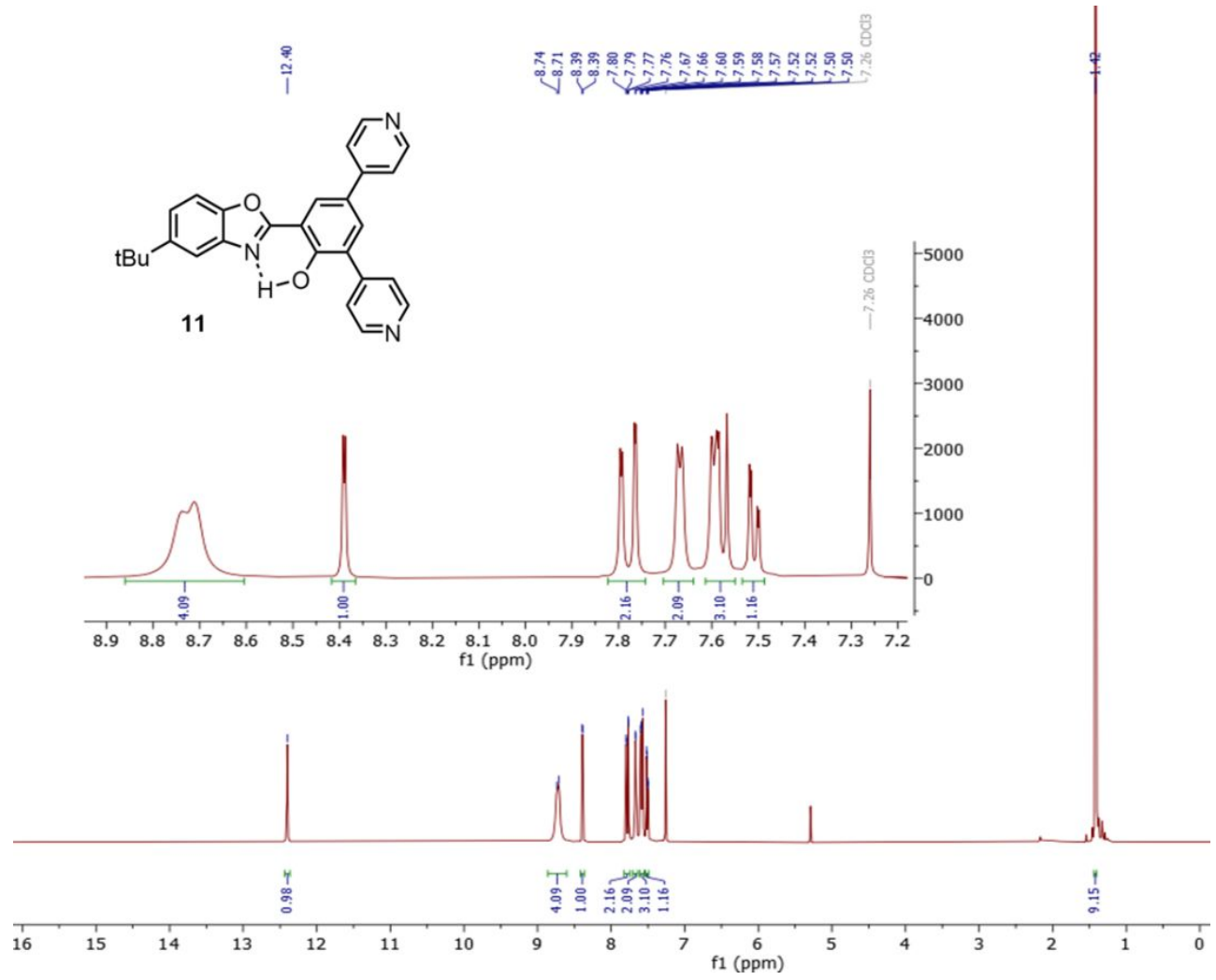

Figure S23. ${ }^{1} \mathrm{H} \mathrm{NMR}\left(\mathrm{CDCl}_{3}, 500 \mathrm{MHz}\right)$, spectrum of $\mathrm{HBO} 11$
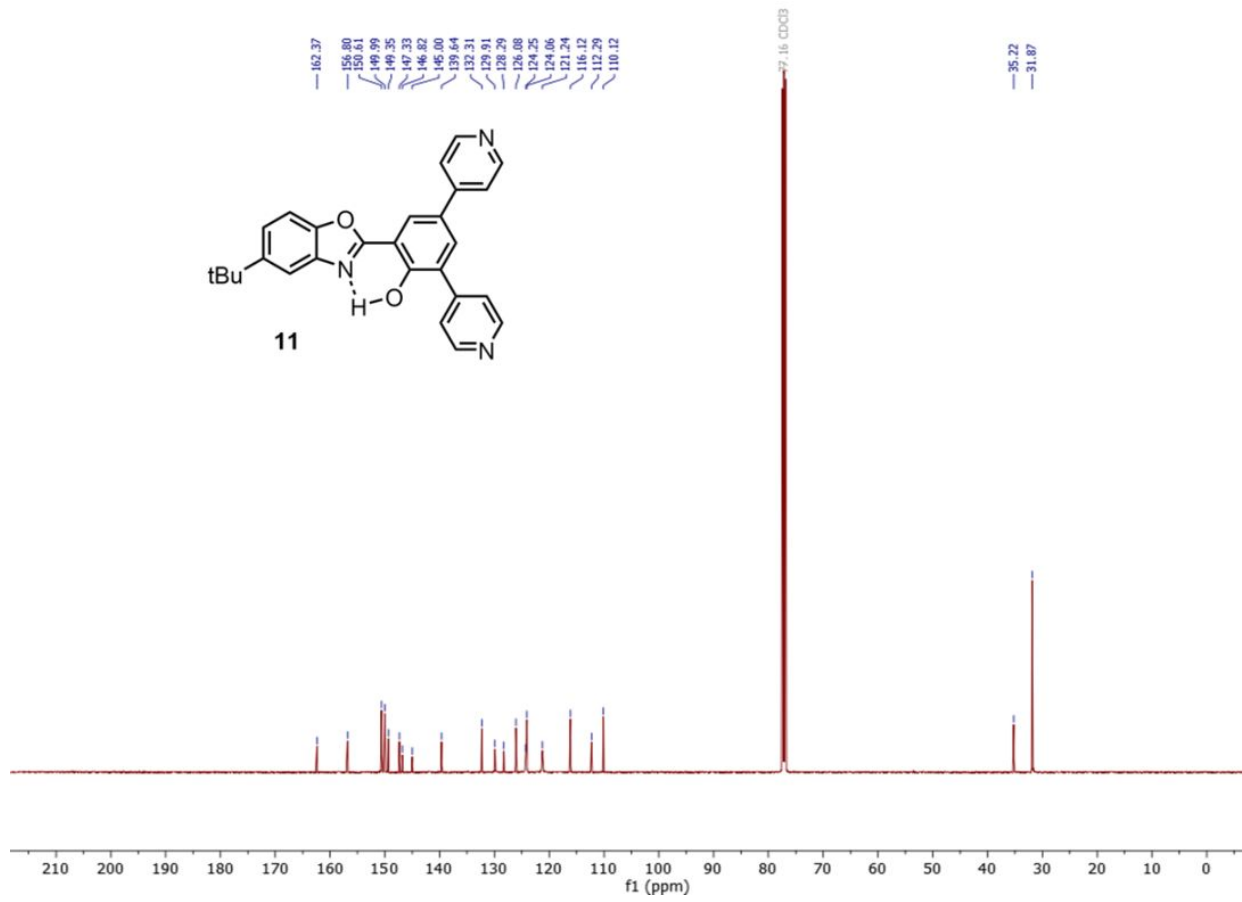

Figure S24. $\left.{ }^{13} \mathrm{C} \mathrm{NMR} \mathrm{(CDCl}, 126 \mathrm{MHz}\right)$, spectrum of $\mathrm{HBO} 11$ 


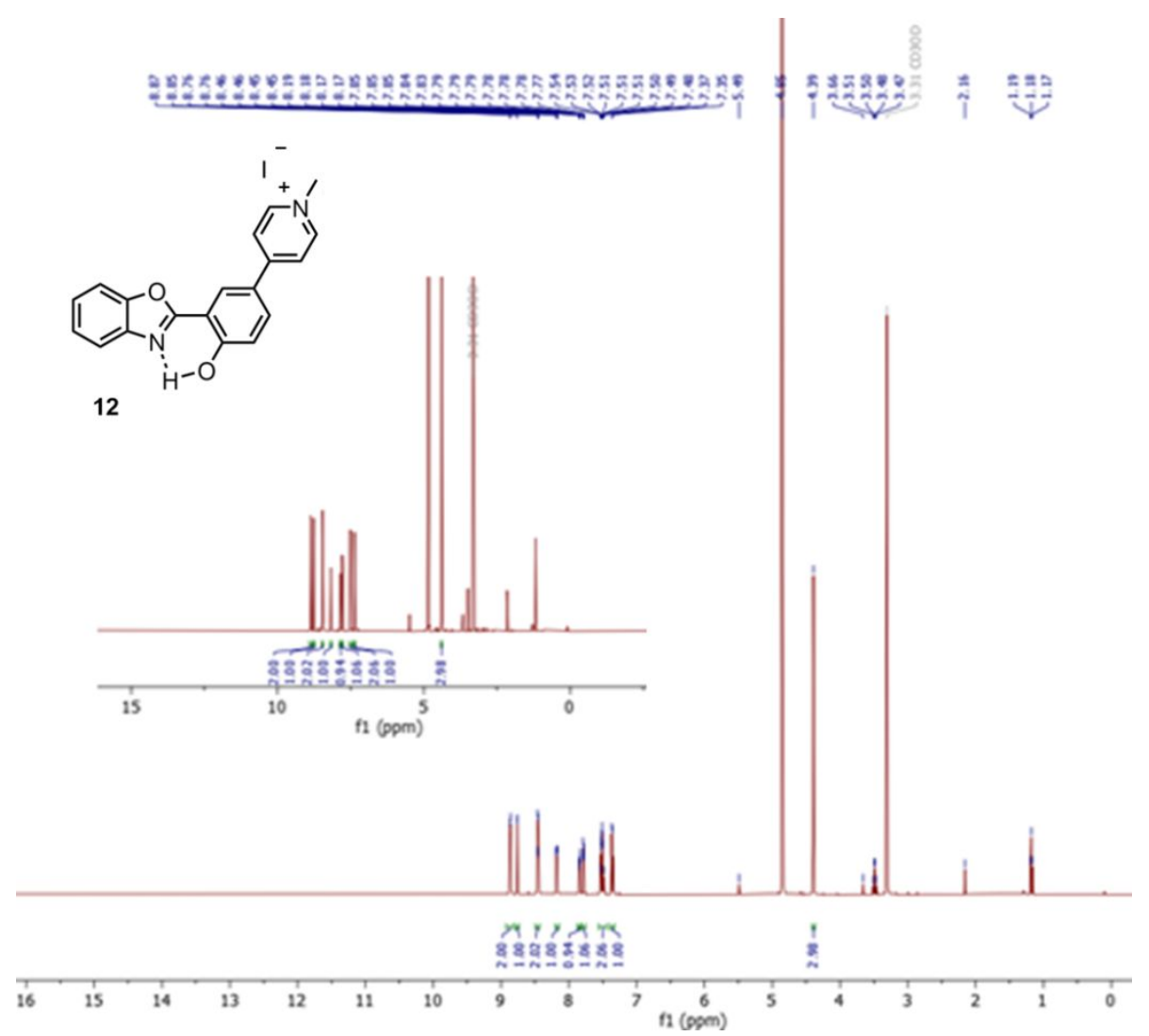

Figure S25. ${ }^{1} \mathrm{H}$ NMR (MeOD, $500 \mathrm{MHz}$ ) spectrum of HBO 12

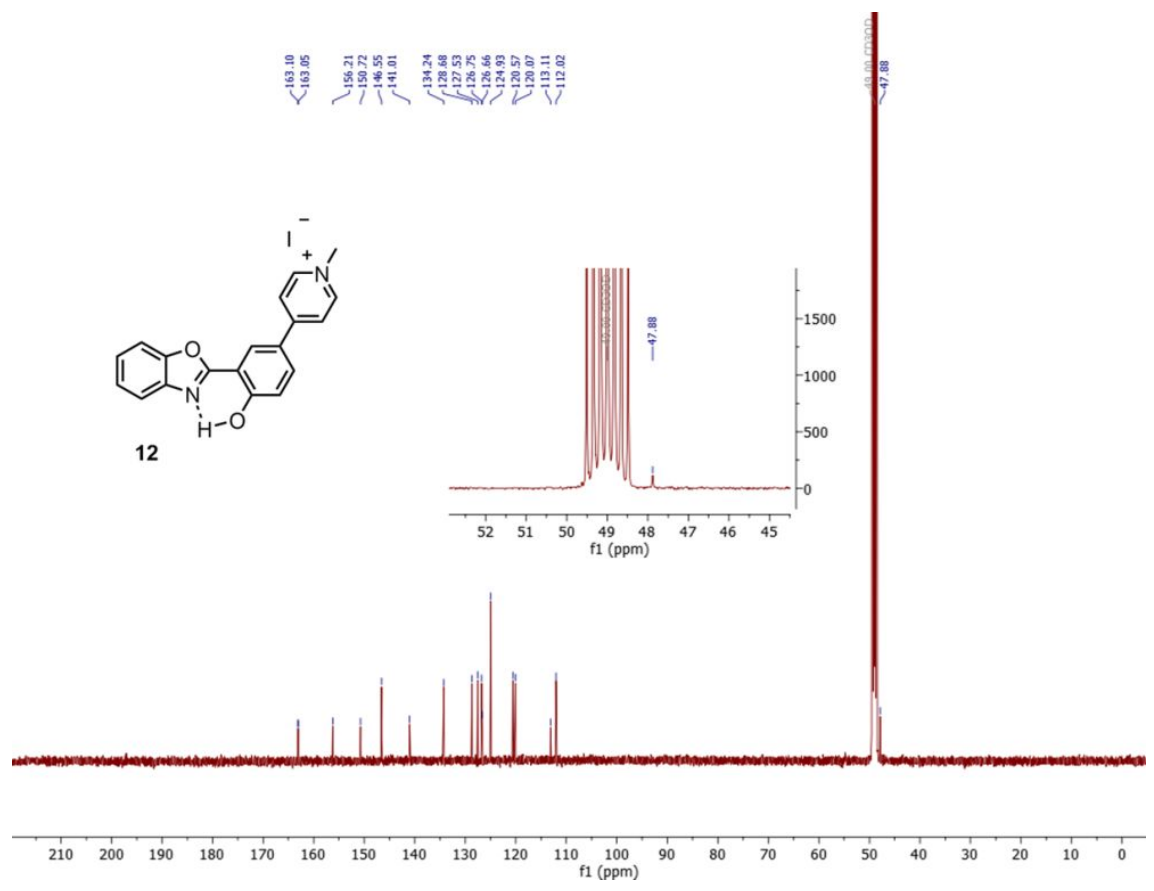

Figure S26. ${ }^{13} \mathrm{C}$ NMR (MeOD, $126 \mathrm{MHz}$ ) spectrum of HBO 12 


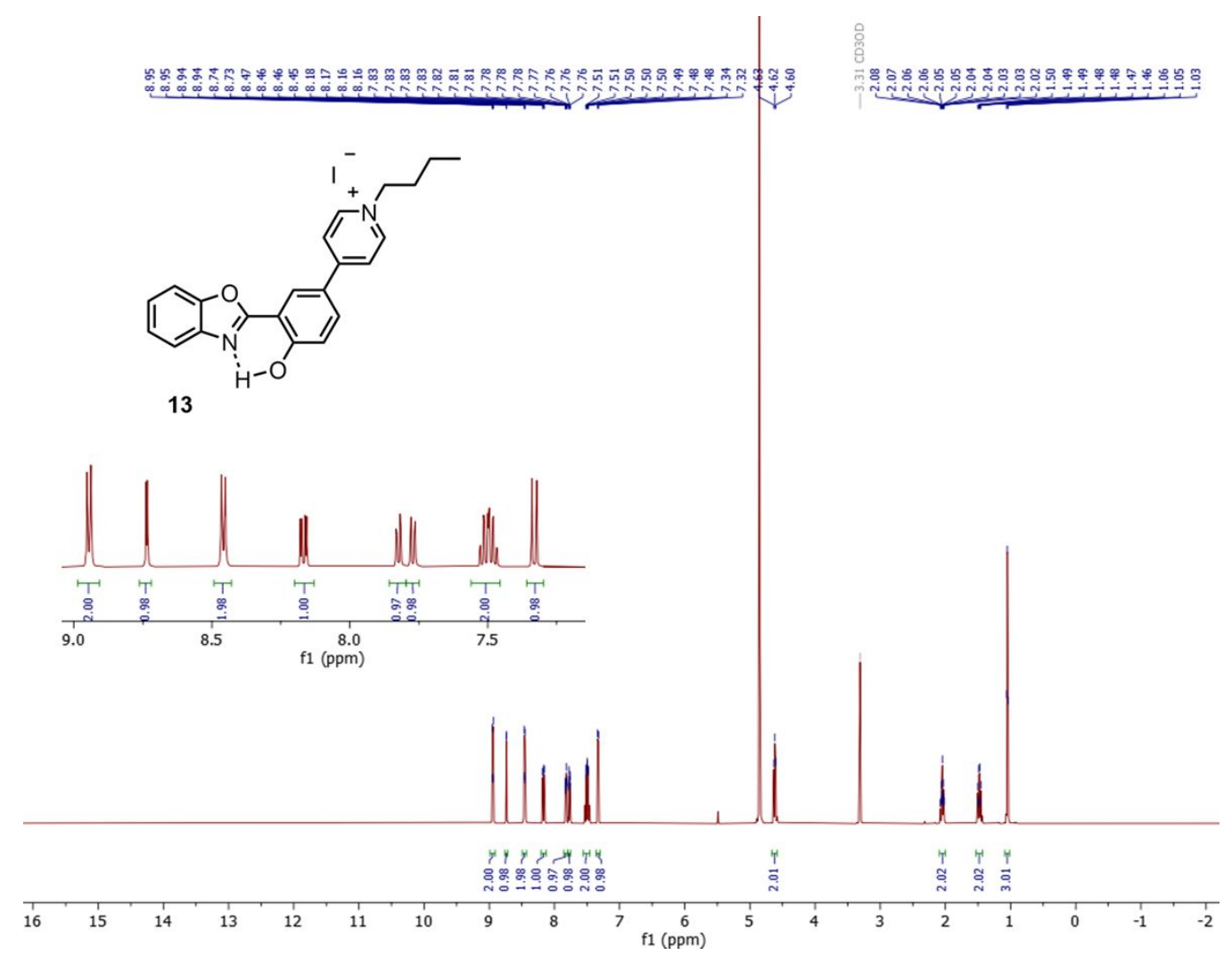

Figure S27. ${ }^{1} \mathrm{H}$ NMR (MeOD, $500 \mathrm{MHz}$ ) spectrum of HBO 13
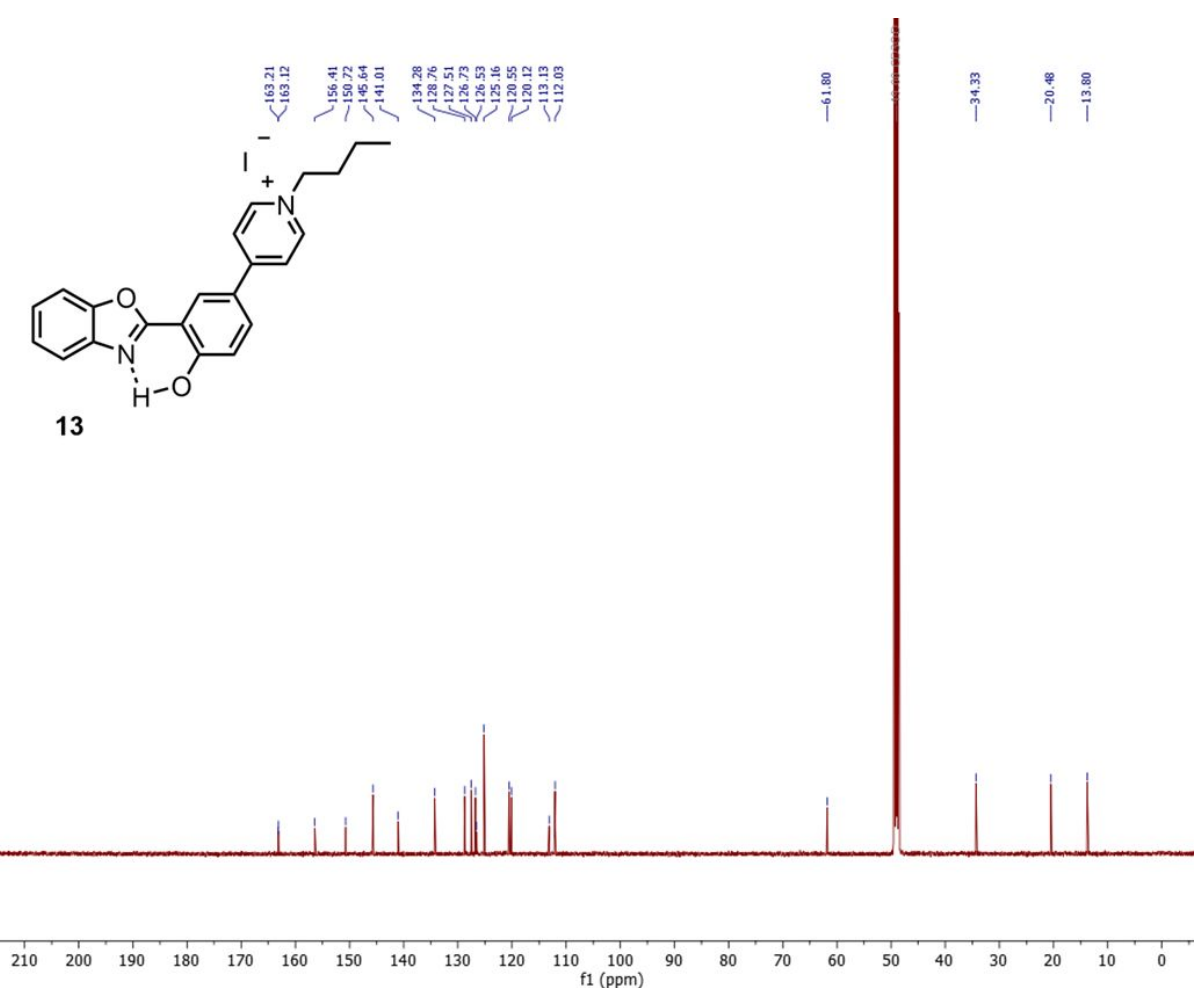

Figure S28. ${ }^{13} \mathrm{C}$ NMR (MeOD, $126 \mathrm{MHz}$ ) spectrum of HBO 13 


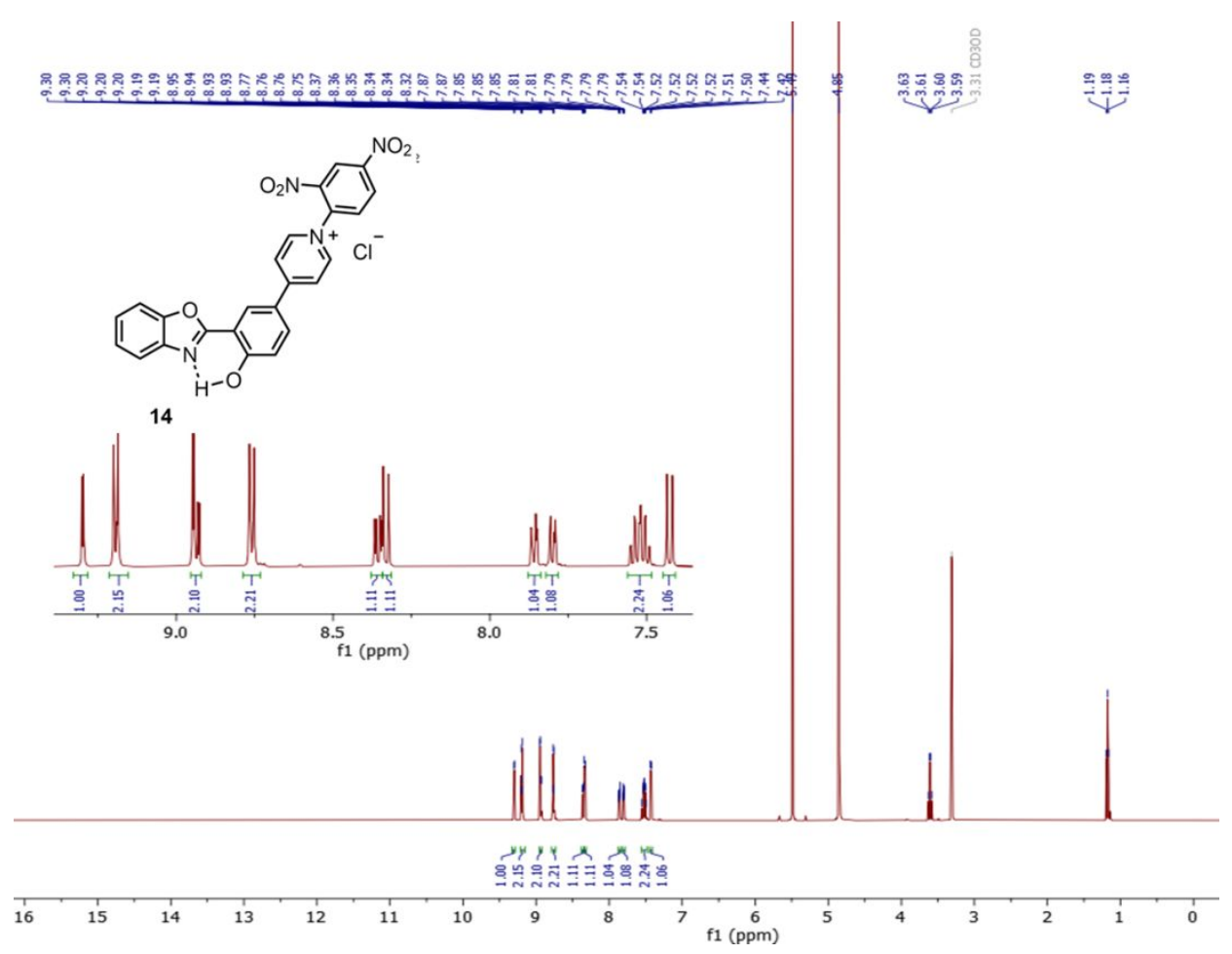

Figure S29. ${ }^{1} \mathrm{H}$ NMR (MeOD, $500 \mathrm{MHz}$ ) spectrum of $\mathrm{HBO} 14$
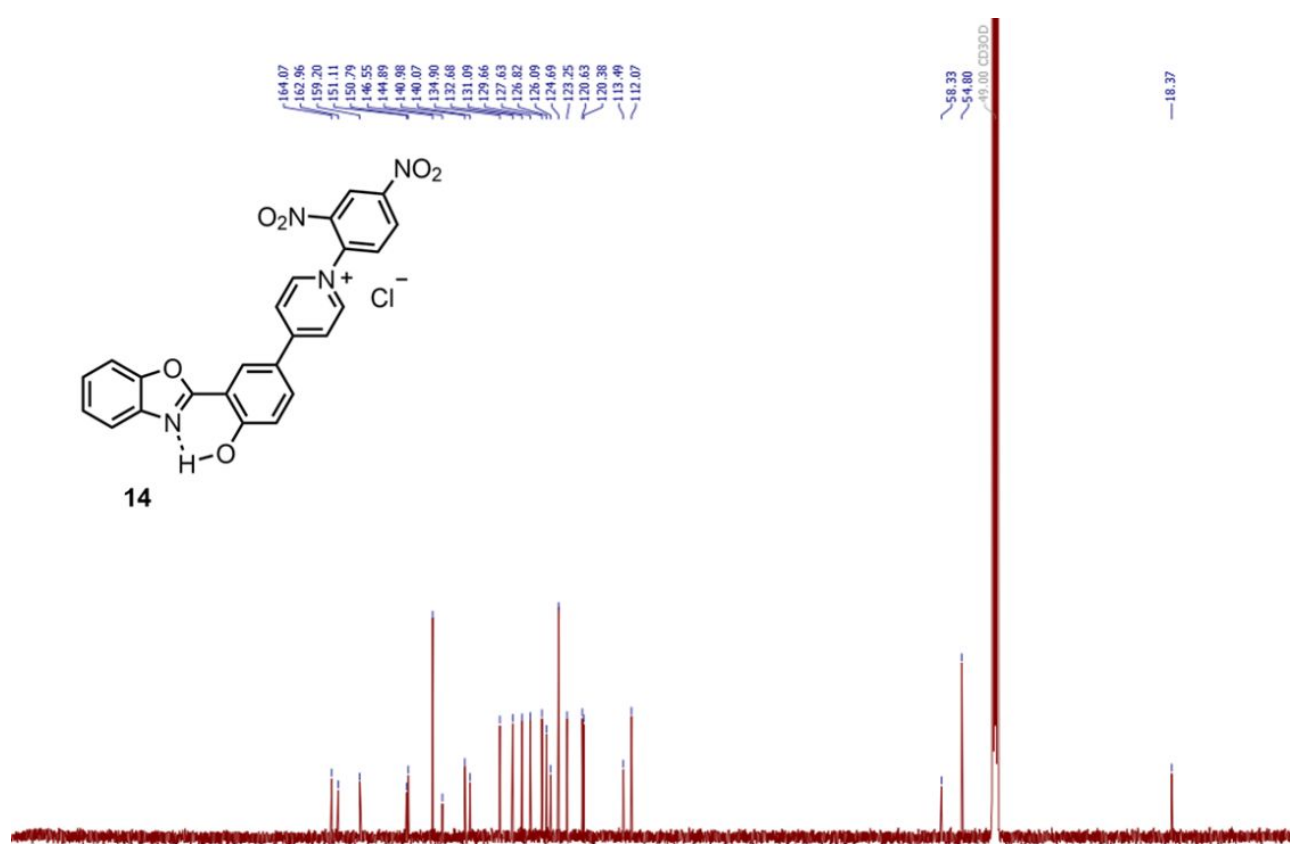

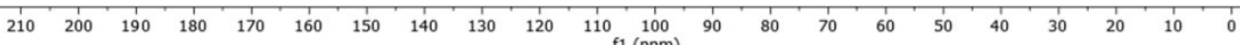

Figure S30. ${ }^{13} \mathrm{C}$ NMR (MeOD, $126 \mathrm{MHz}$ ) spectrum of HBO 14 


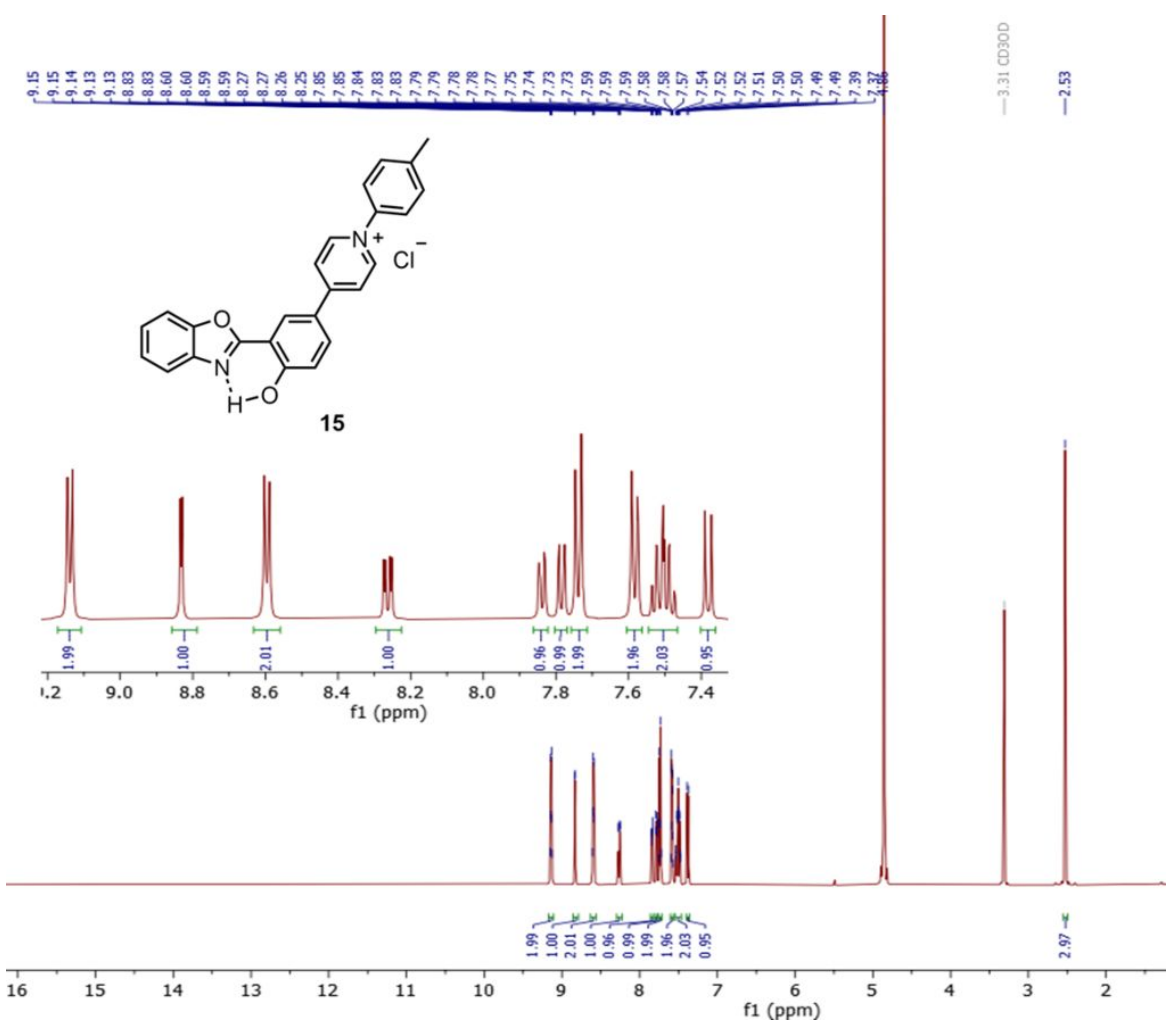

Figure S31. ${ }^{1} \mathrm{H}$ NMR (MeOD, $500 \mathrm{MHz}$ ) spectrum of HBO 15
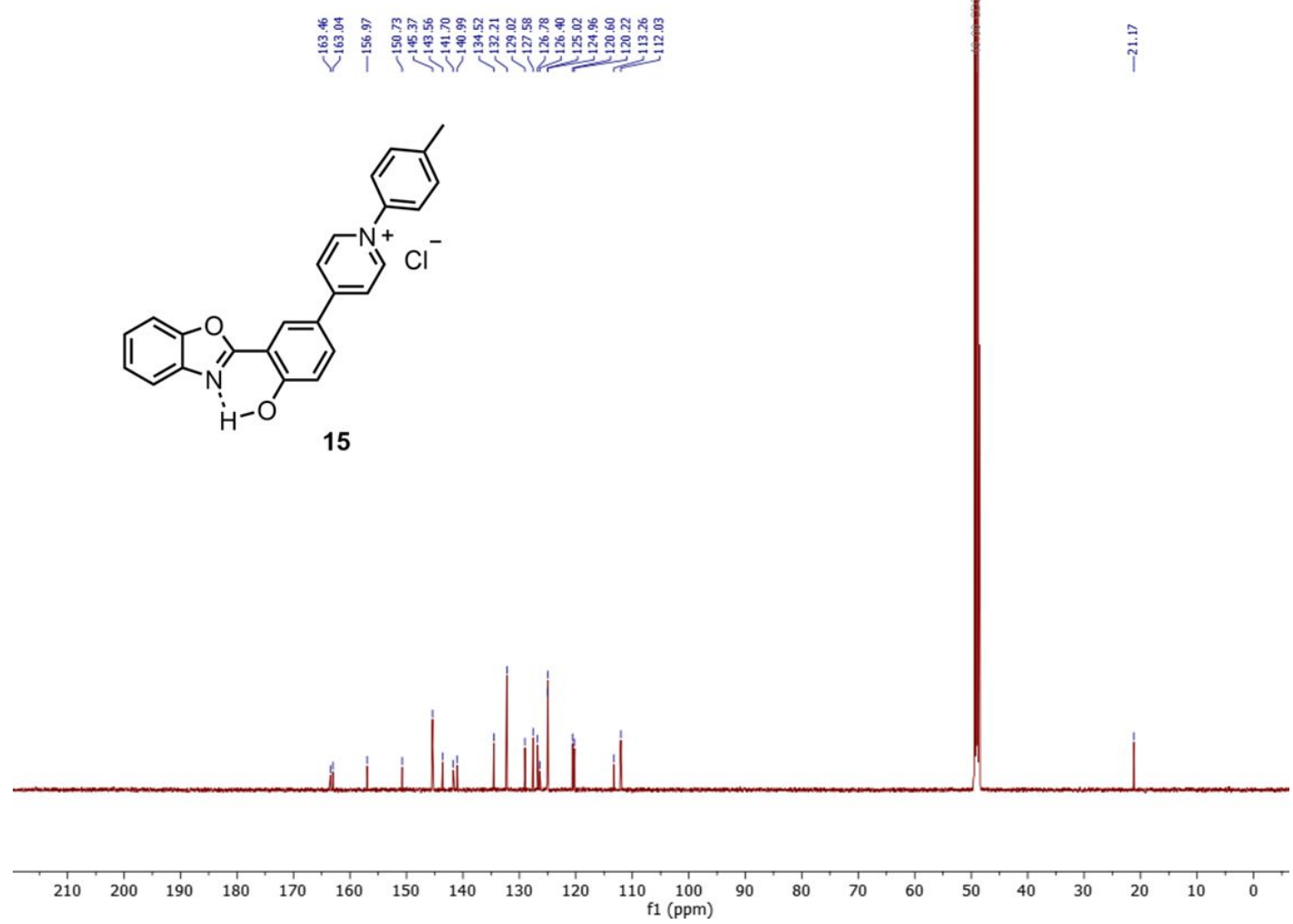

Figure S32. ${ }^{13} \mathrm{C}$ NMR (MeOD, $126 \mathrm{MHz}$ ) spectrum of HBO 15 


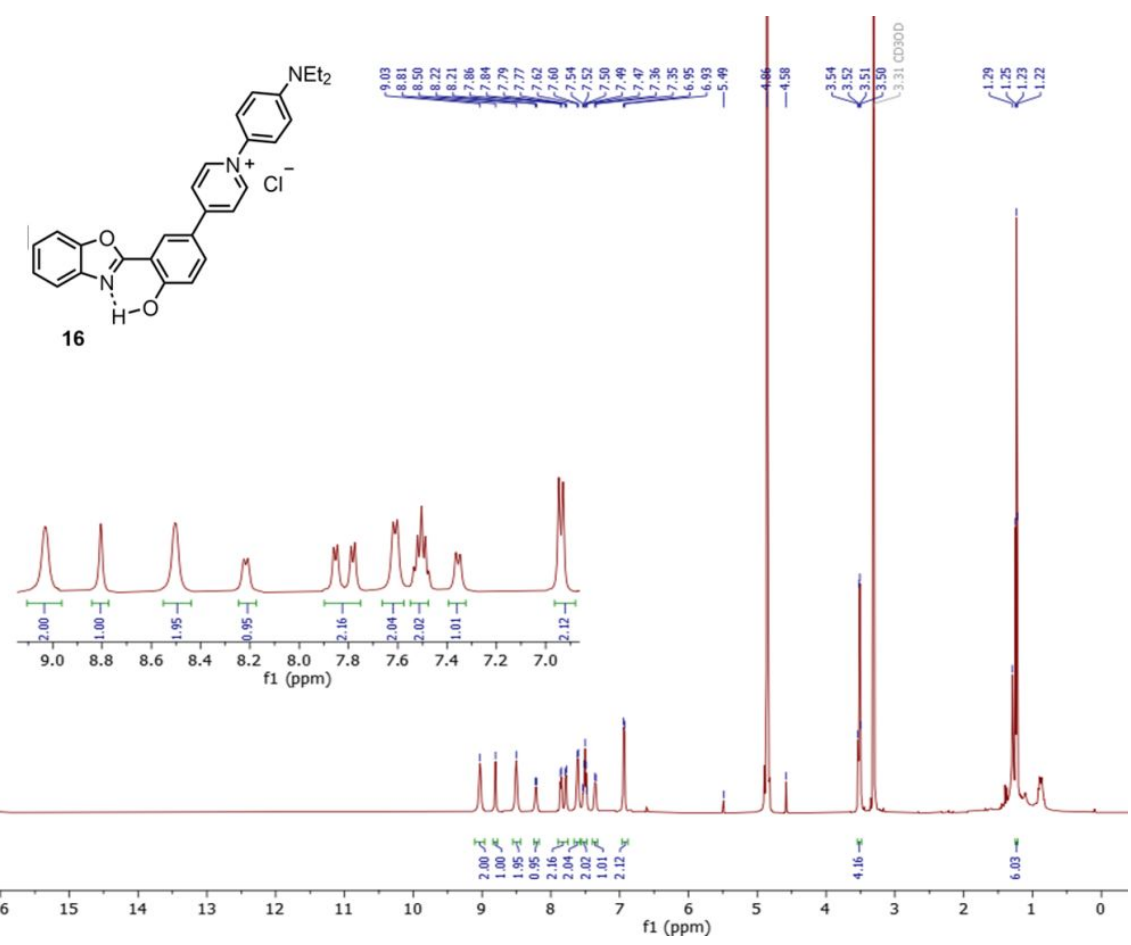

Figure S33. ${ }^{1} \mathrm{H}$ NMR (MeOD, $500 \mathrm{MHz}$ ) spectrum of $\mathrm{HBO} 16$
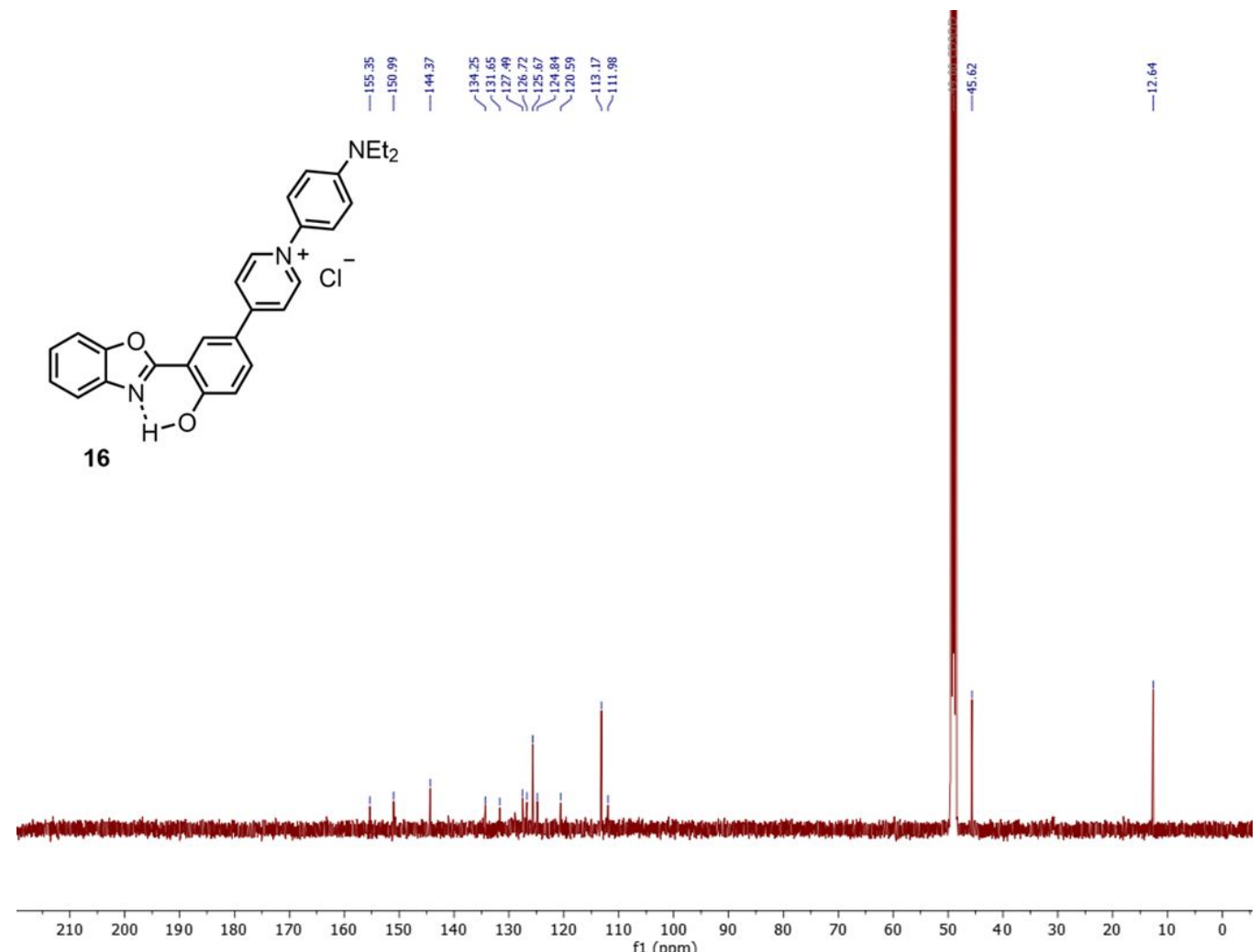

Figure S34. ${ }^{13} \mathrm{C}$ NMR (MeOD, $126 \mathrm{MHz}$ ) spectrum of HBO 16 


\section{S3 Spectroscopic data}

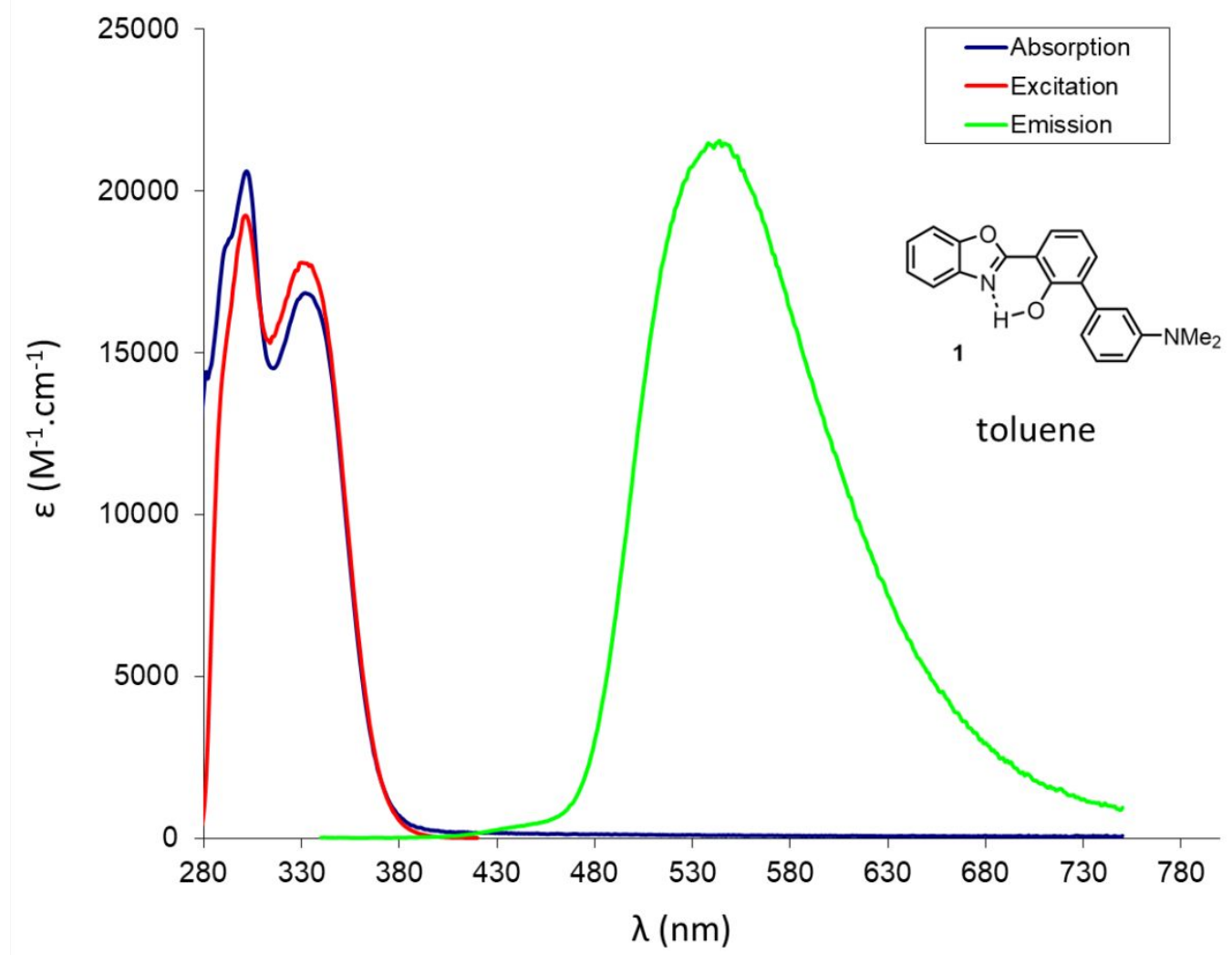

Figure S35. Absorption (blue), Emission (green) and Excitation (red) spectra of HBO 1 in toluene.

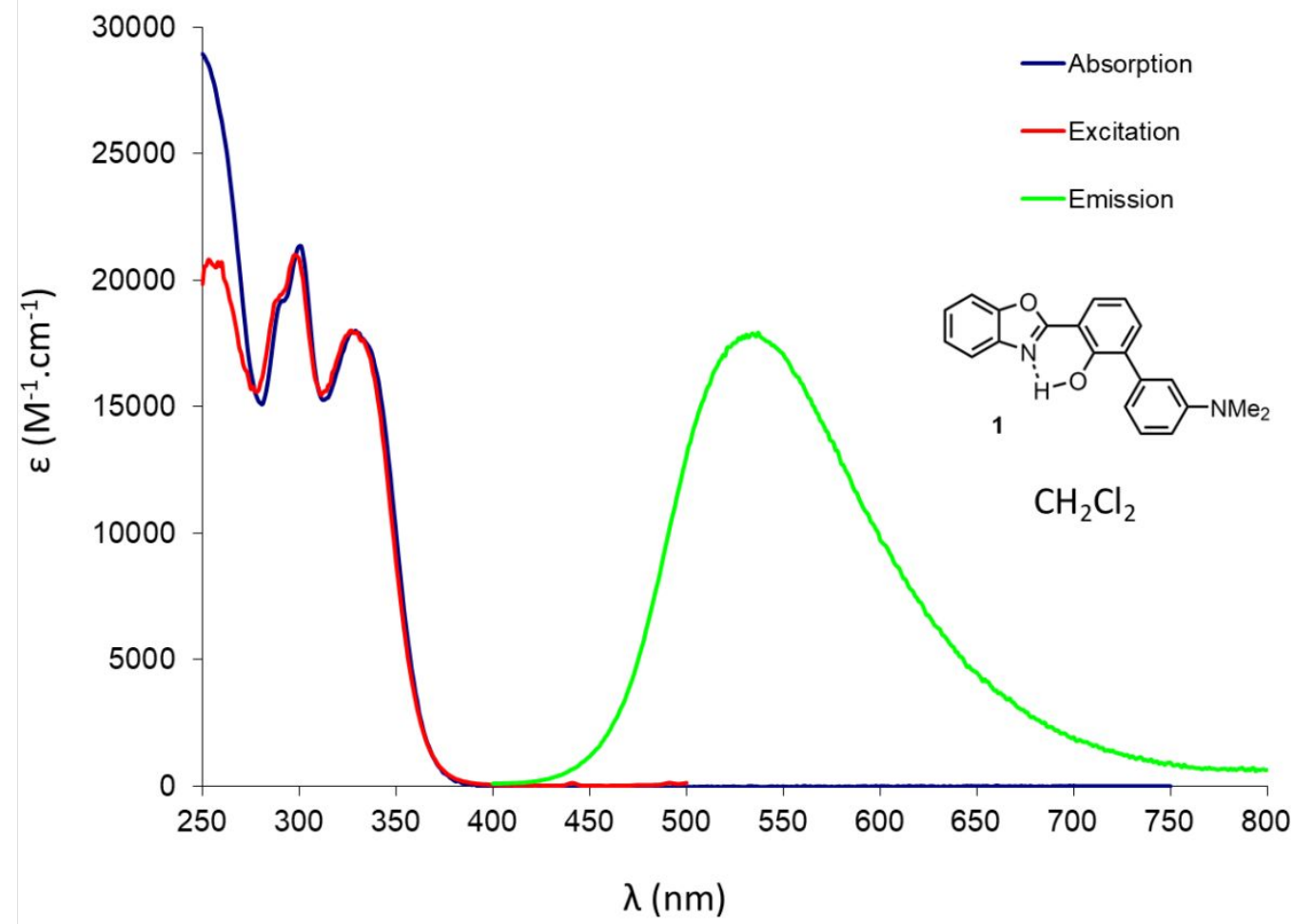

Figure S36. Absorption (blue), Emission (green) and Excitation (red) spectra of $\mathrm{HBO} 1$ in $\mathrm{CH}_{2} \mathrm{Cl}_{2}$. 


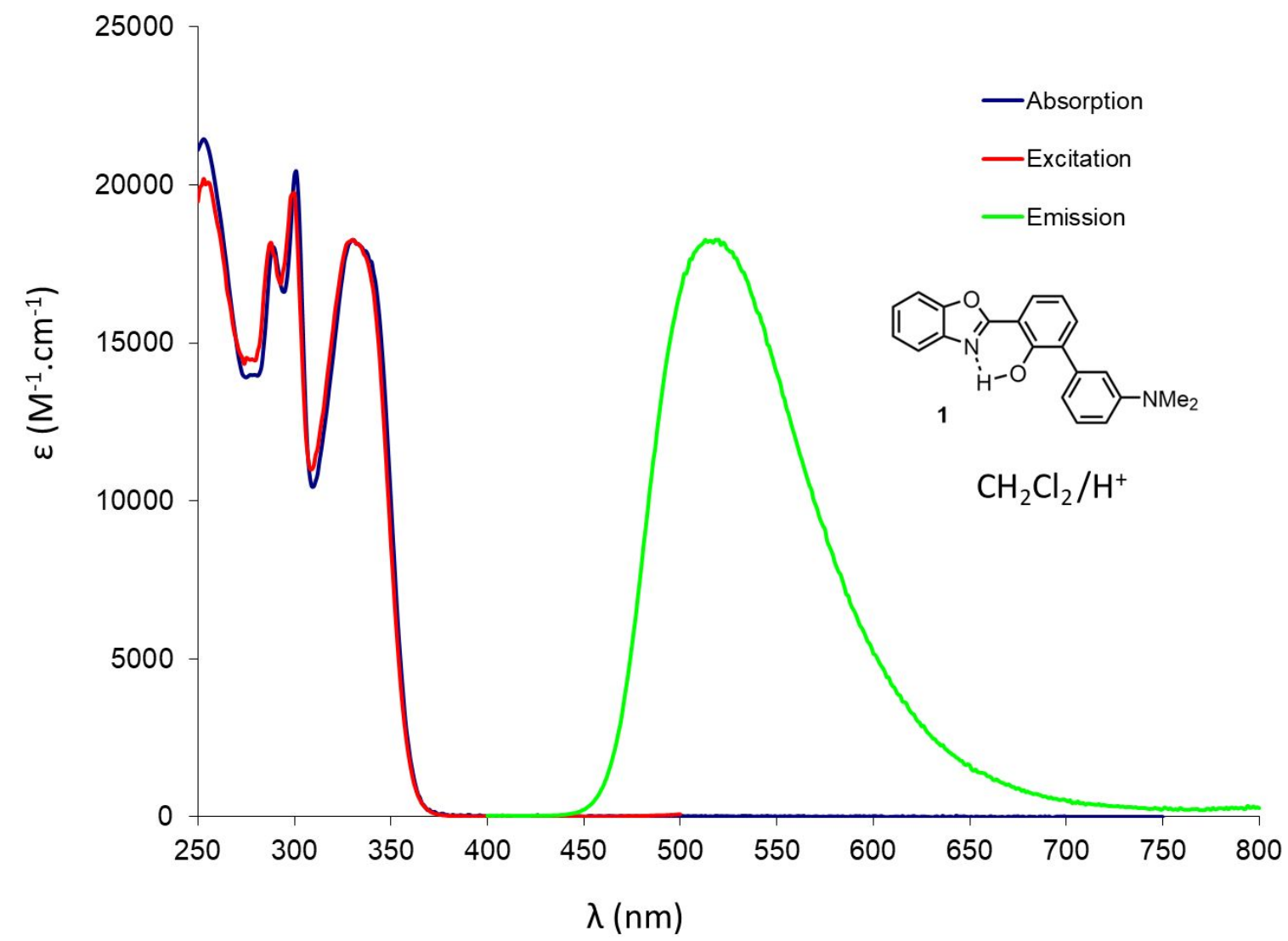

Figure S37. Absorption (blue), Emission (green) and Excitation (red) spectra of HBO 1 in protonated $\mathrm{CH}_{2} \mathrm{Cl}_{2}$.

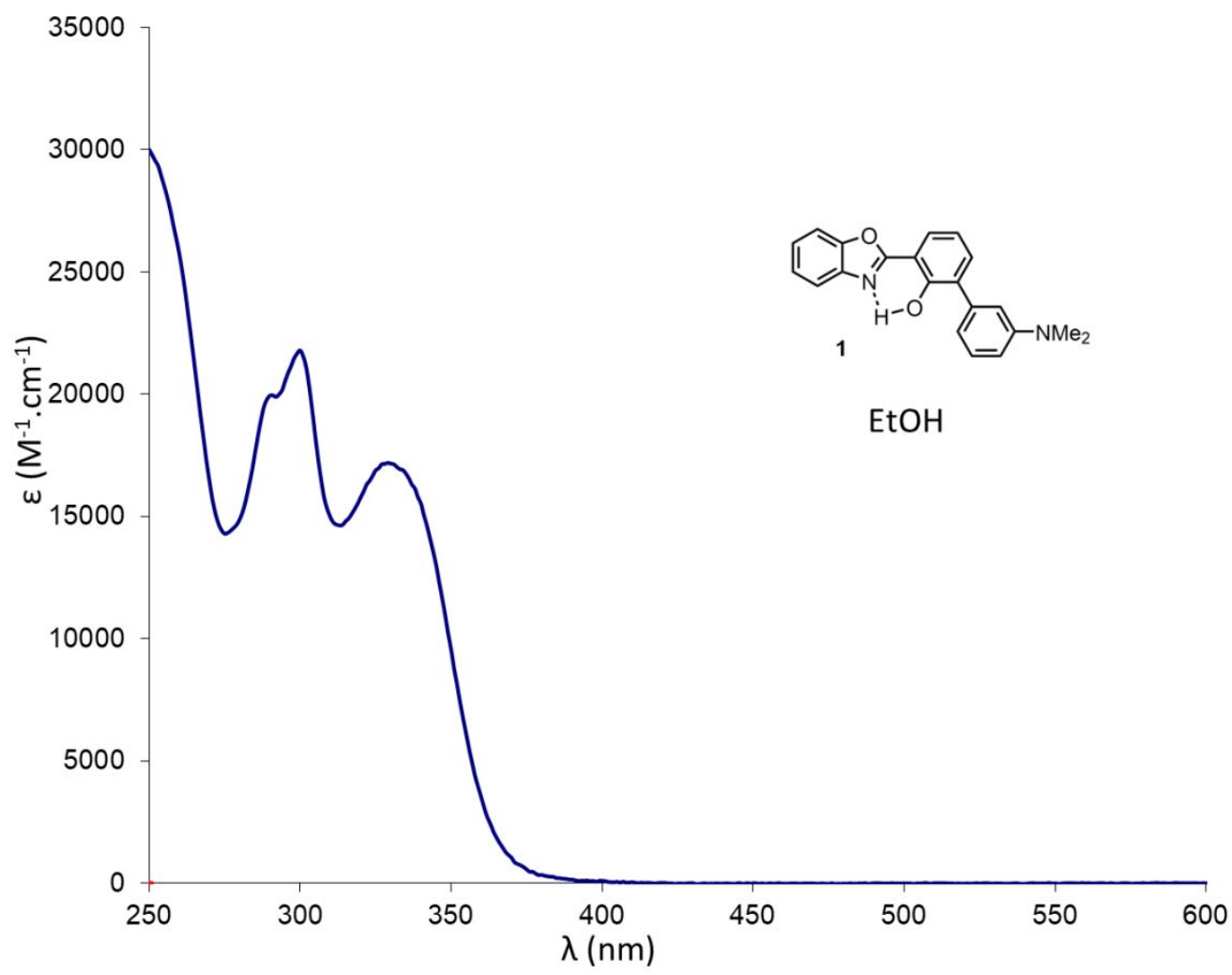

Figure S38. Absorption spectrum of $\mathrm{HBO} 1$ in EtOH. 


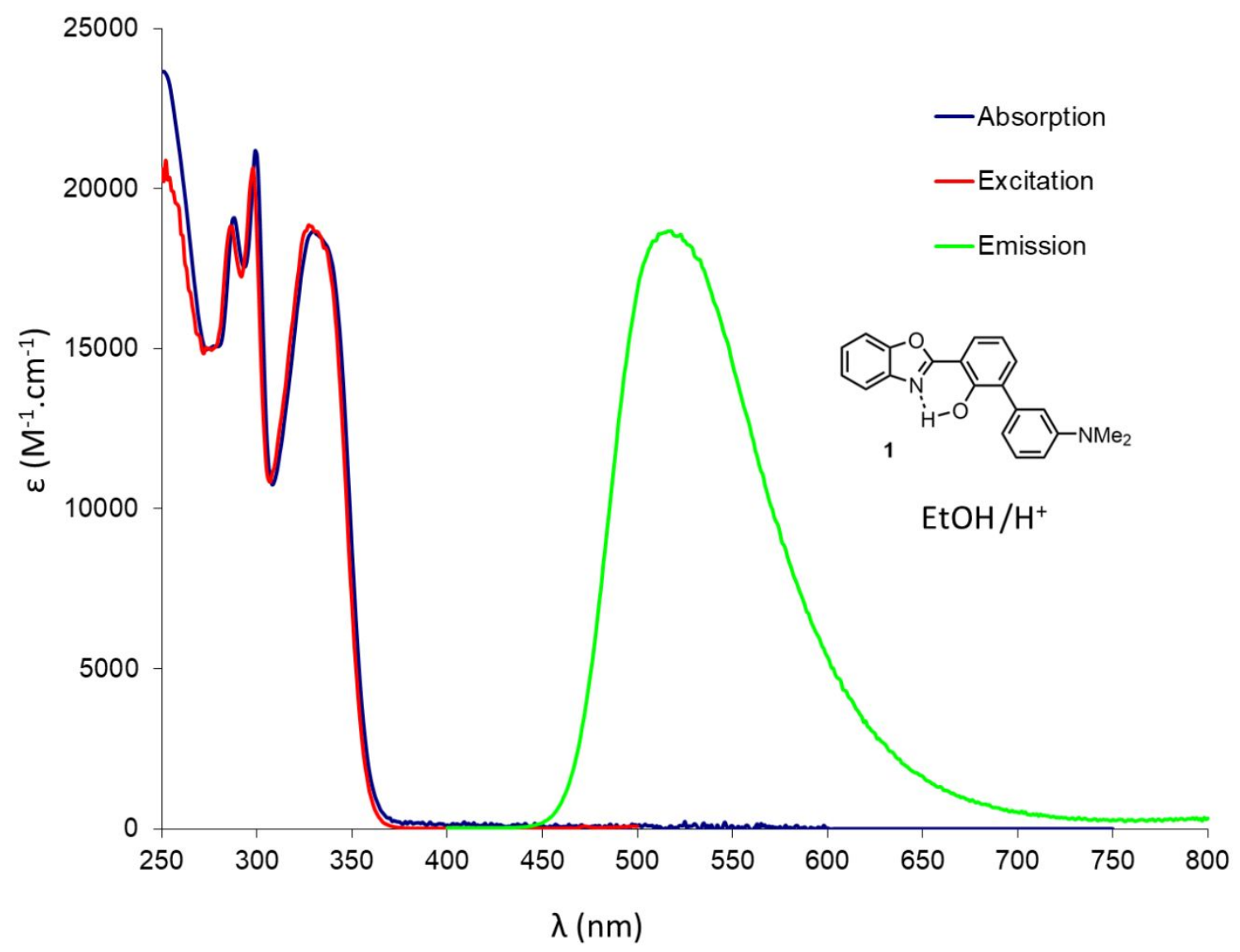

Figure S39. Absorption (blue), Emission (green) and Excitation (red) spectra of HBO 1 in protonated EtOH.

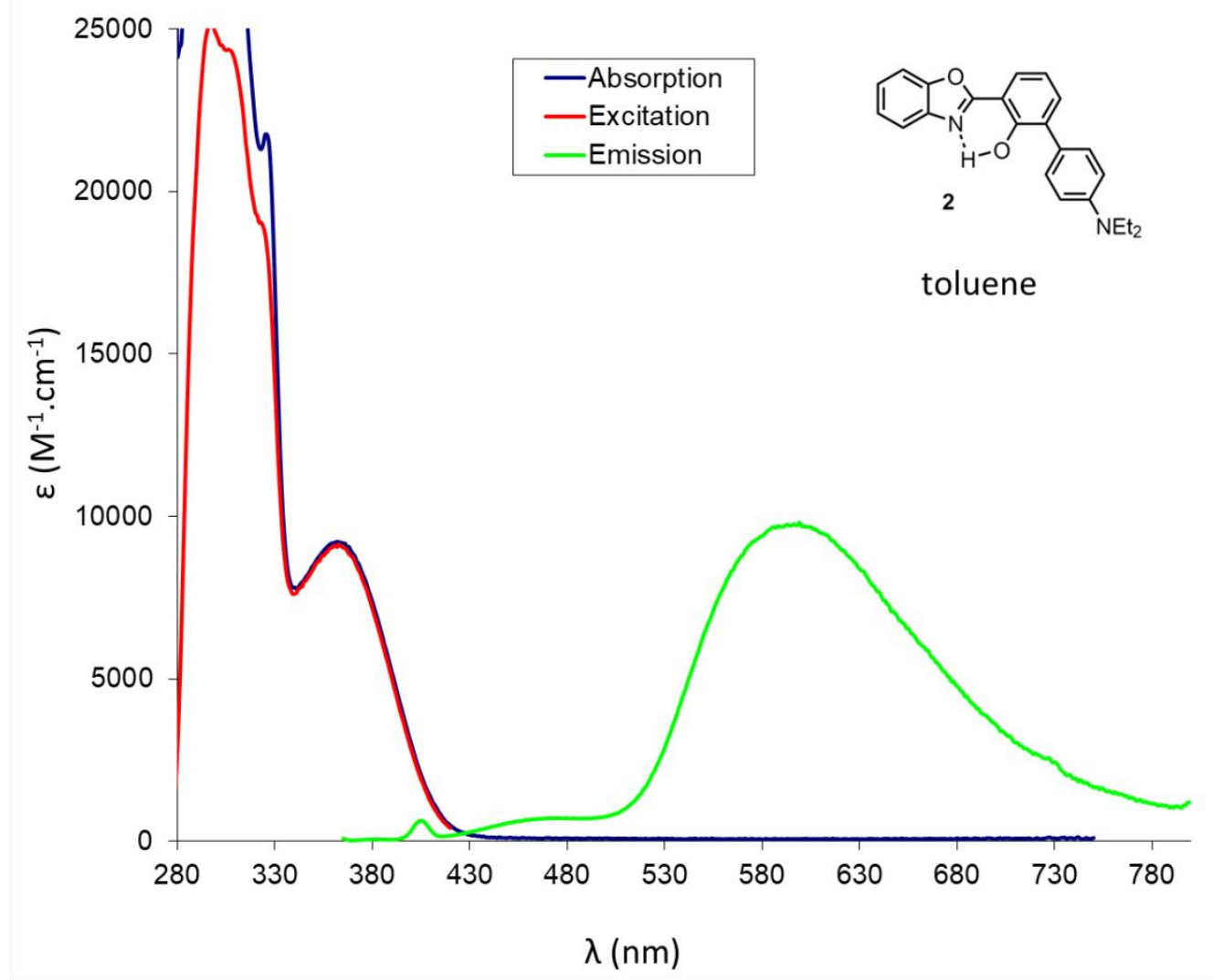

Figure S40. Absorption (blue), Emission (green) and Excitation (red) spectra of HBO 2 in toluene. 


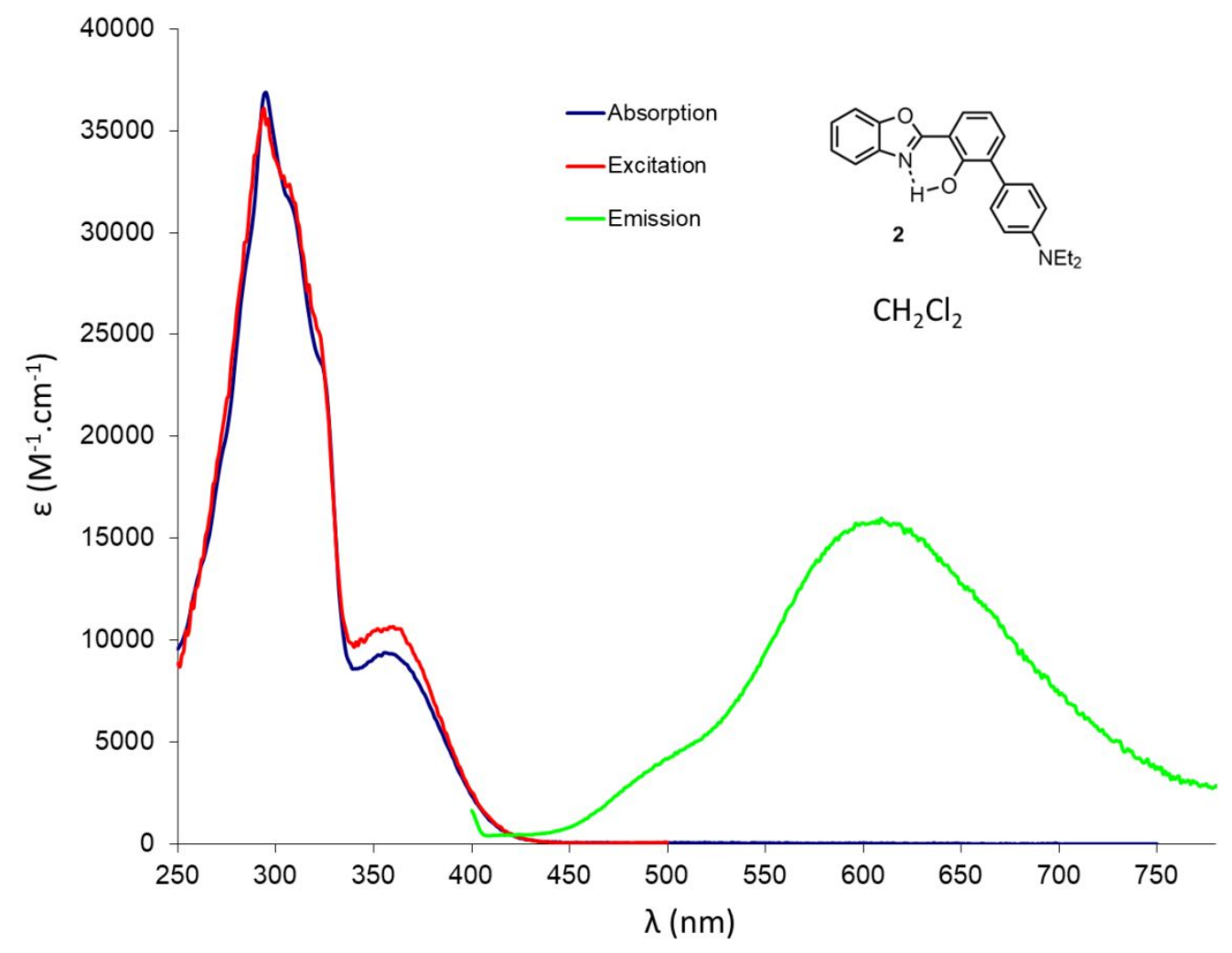

Figure S41. Absorption (blue), Emission (green) and Excitation (red) spectra of $\mathrm{HBO} 2$ in $\mathrm{CH}_{2} \mathrm{Cl}_{2}$.

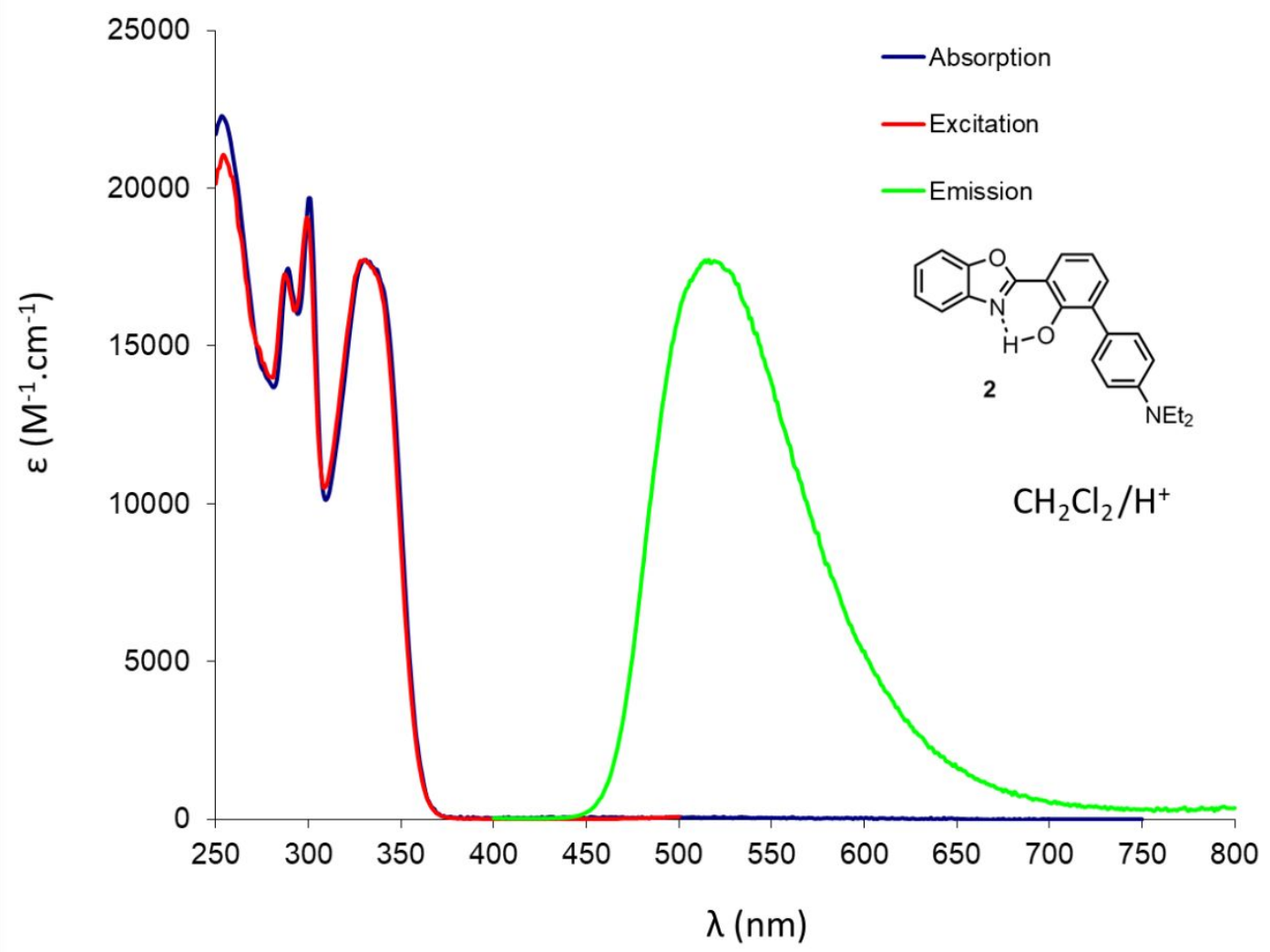

Figure S42. Absorption (blue), Emission (green) and Excitation (red) spectra of $\mathrm{HBO} 2$ in protonated $\mathrm{CH}_{2} \mathrm{Cl}_{2}$. 


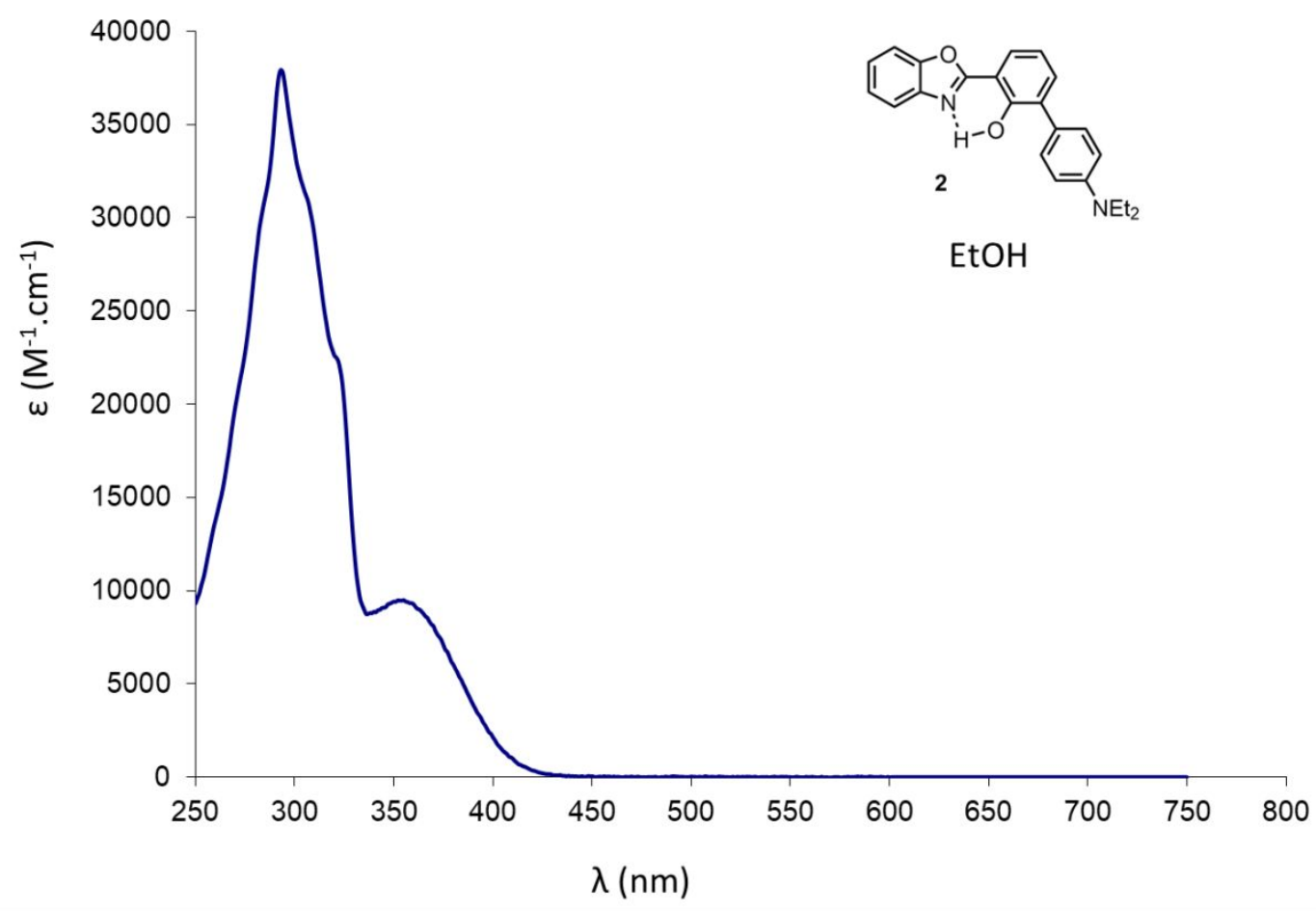

Figure S43. Absorption spectrum of HBO 2 in EtOH.

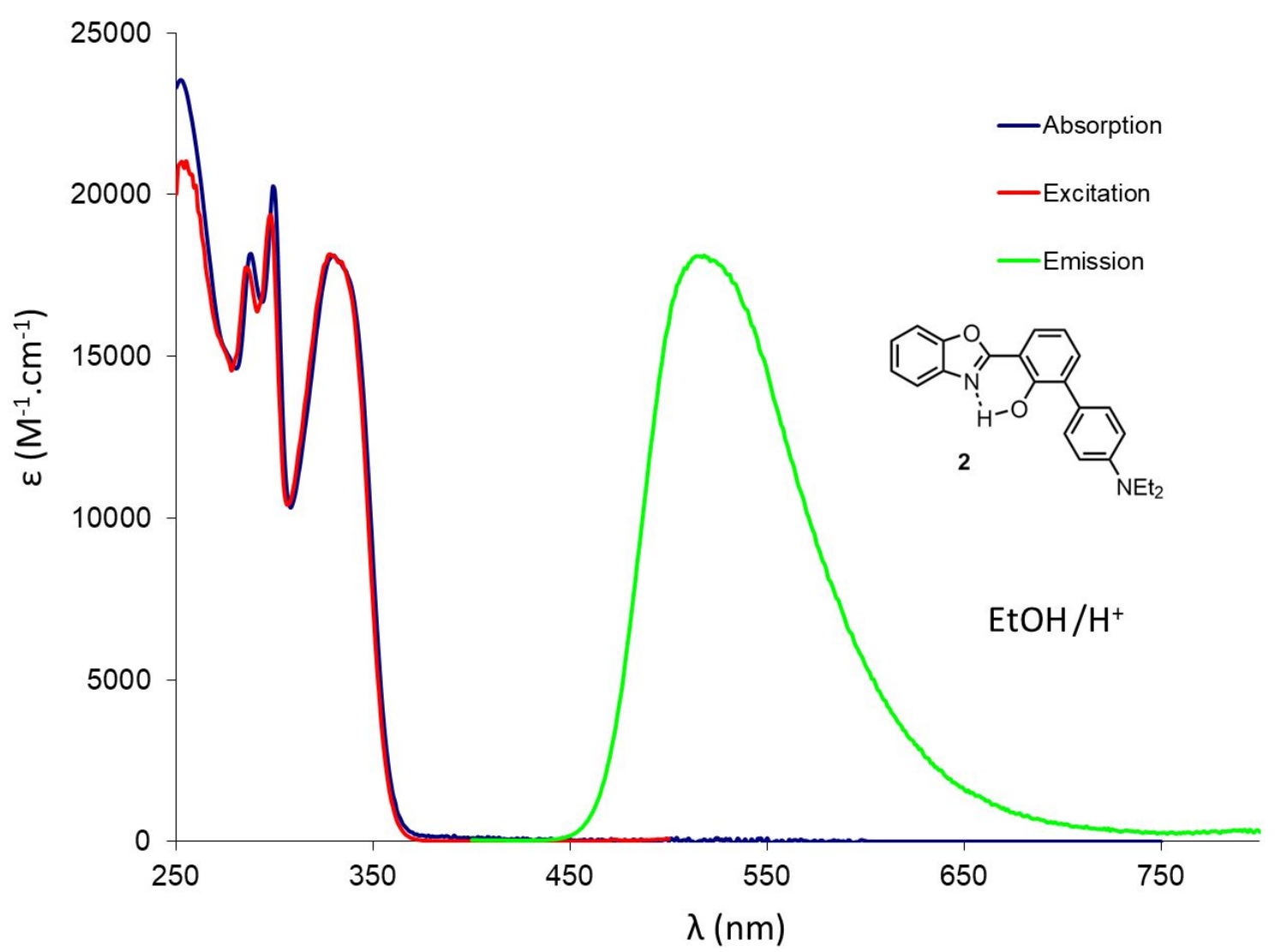

Figure S44. Absorption (blue), Emission (green) and Excitation (red) spectra of $\mathrm{HBO} 2$ in protonated EtOH. 


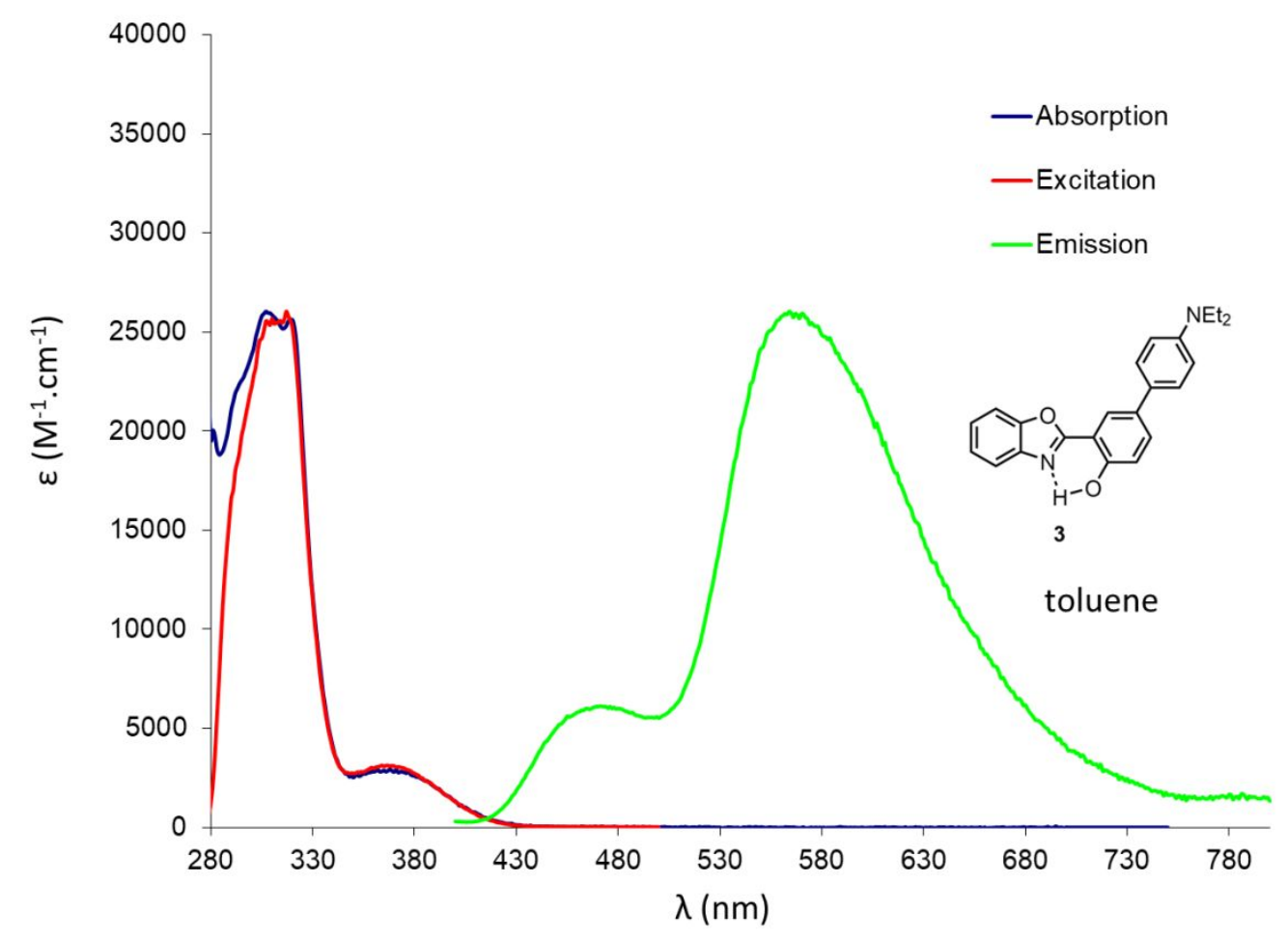

Figure S45. Absorption (blue), Emission (green) and Excitation (red) spectra of HBO 3 in toluene.

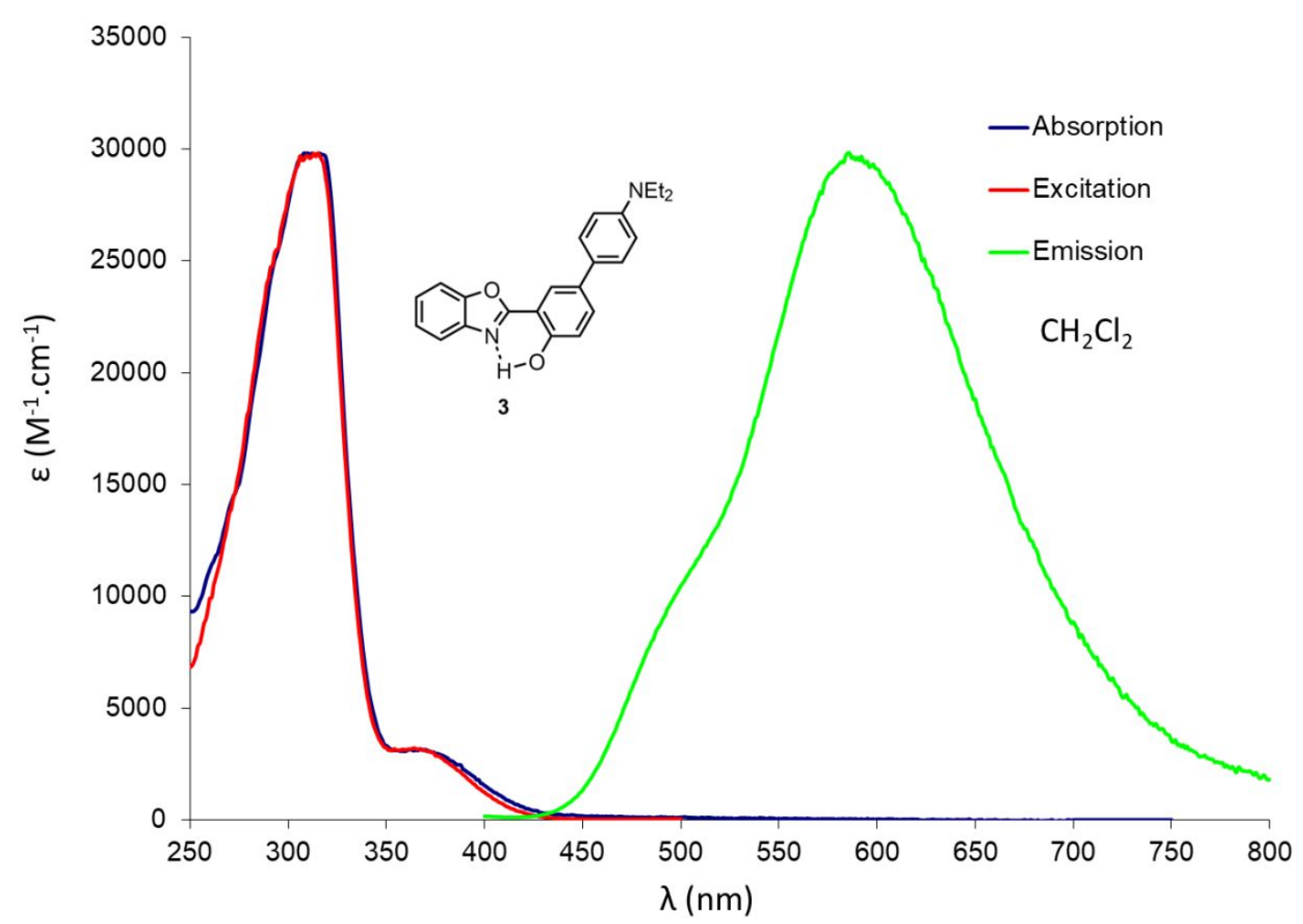

Figure S46. Absorption (blue), Emission (green) and Excitation (red) spectra of $\mathrm{HBO} 3$ in $\mathrm{CH}_{2} \mathrm{Cl}_{2}$. 


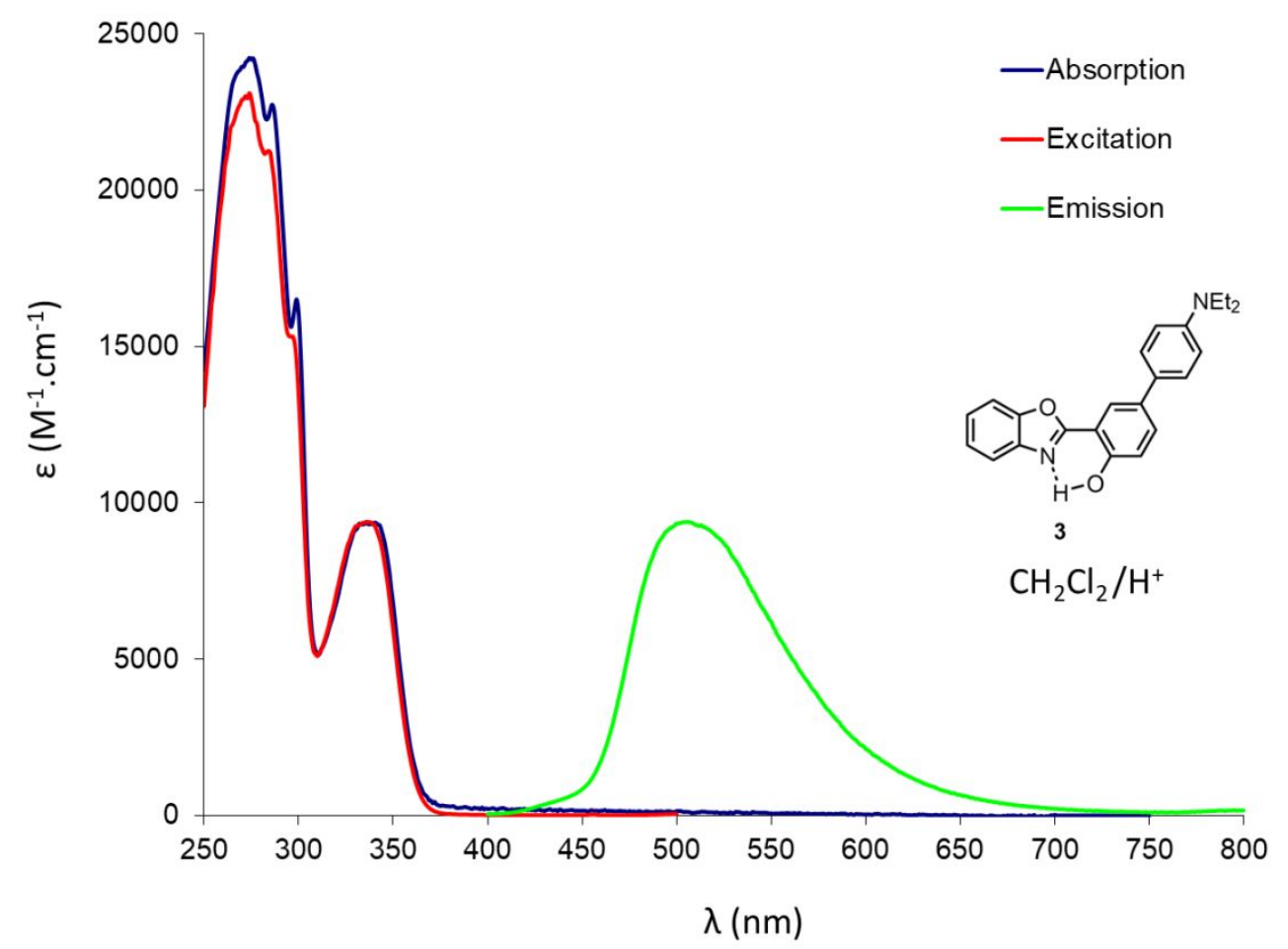

Figure S47. Absorption (blue), Emission (green) and Excitation (red) spectra of $\mathrm{HBO} 3$ in protonated $\mathrm{CH}_{2} \mathrm{Cl}_{2}$.

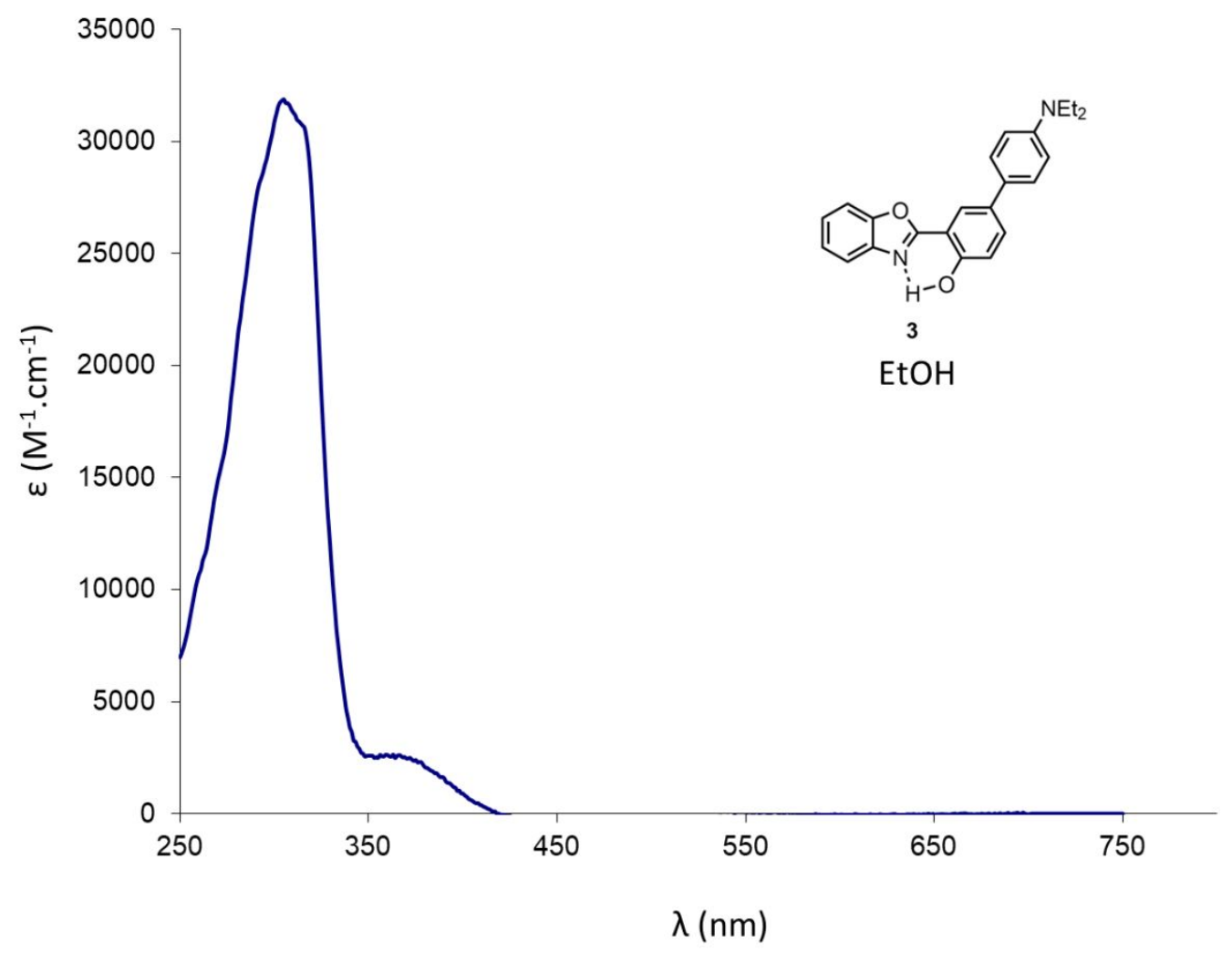

Figure S48. Absorption spectrum of $\mathrm{HBO} 3$ in EtOH. 


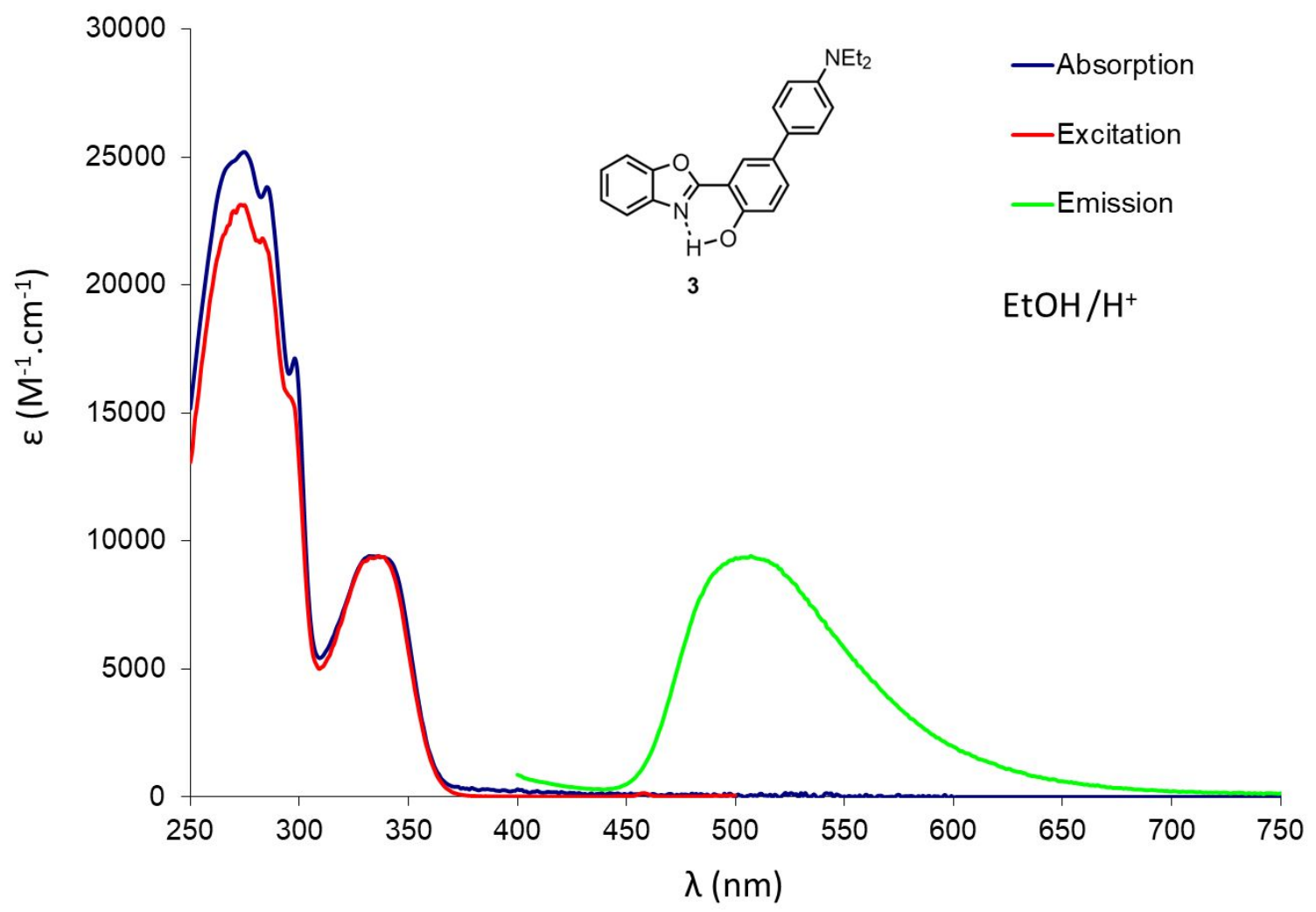

Figure S49. Absorption (blue), Emission (green) and Excitation (red) spectra of HBO 3 in protonated EtOH.

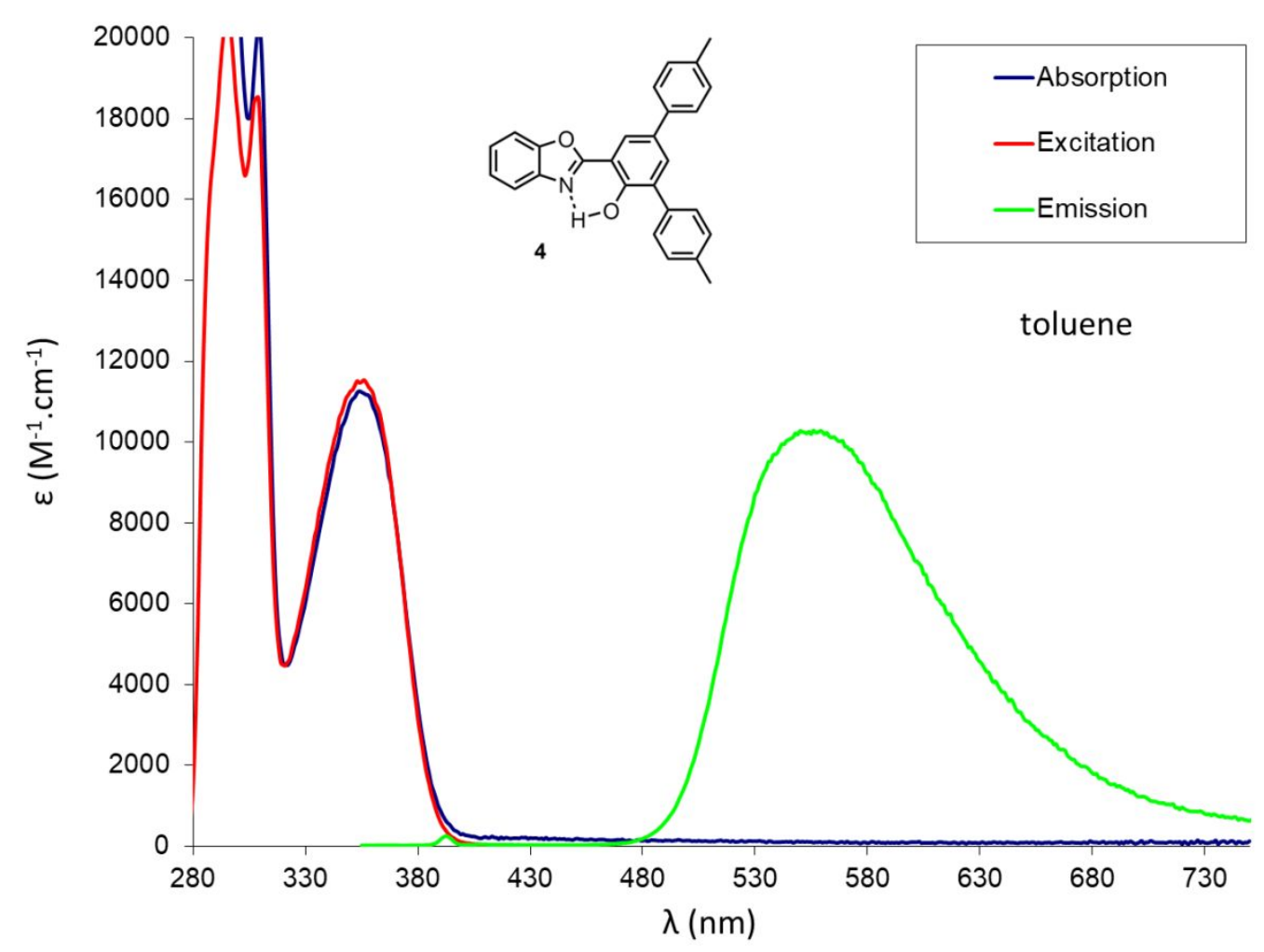

Figure S50. Absorption (blue), Emission (green) and Excitation (red) spectra of HBO 4 in toluene. 


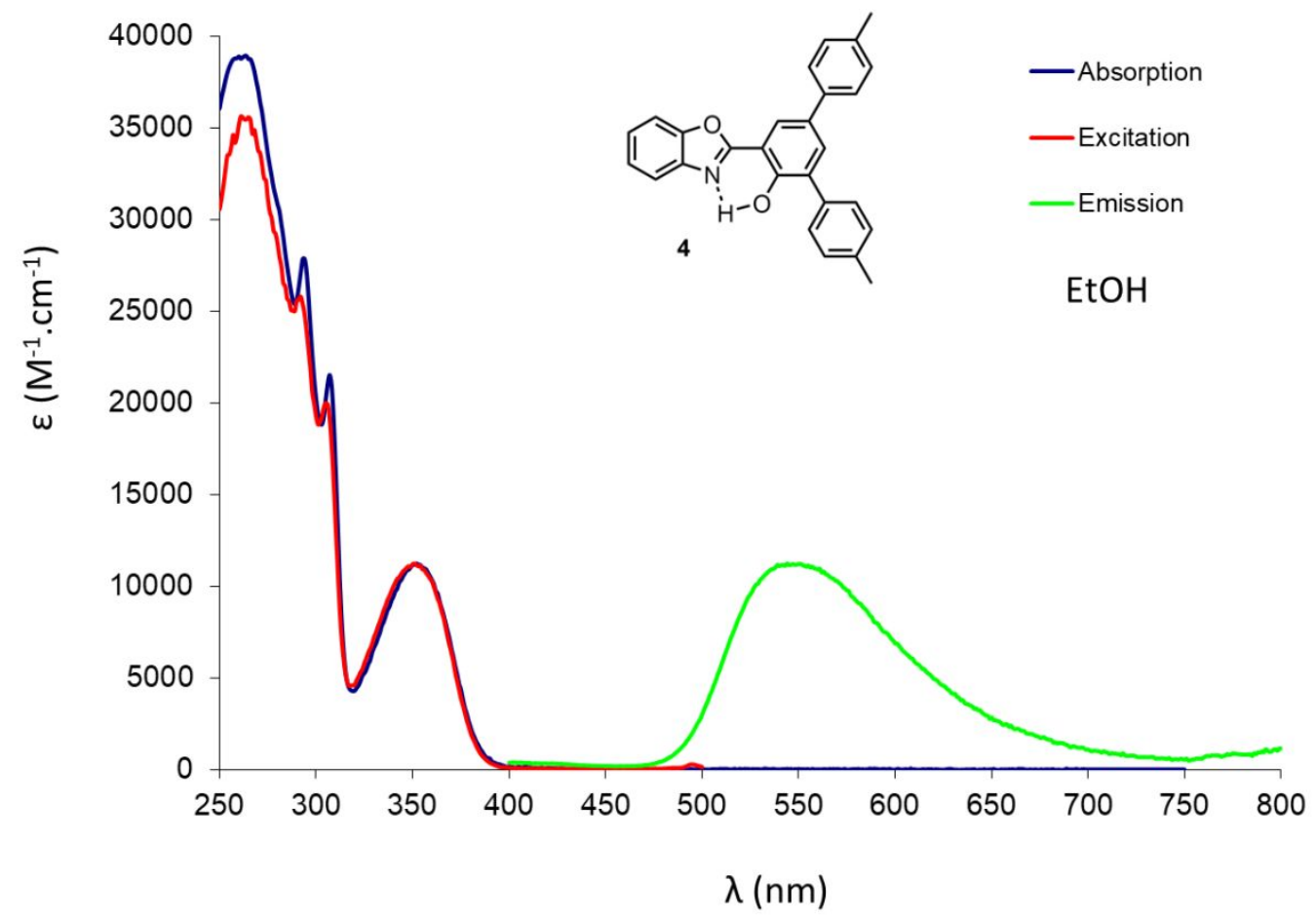

Figure S51. Absorption (blue), Emission (green) and Excitation (red) spectra of $\mathrm{HBO} 4$ in EtOH.

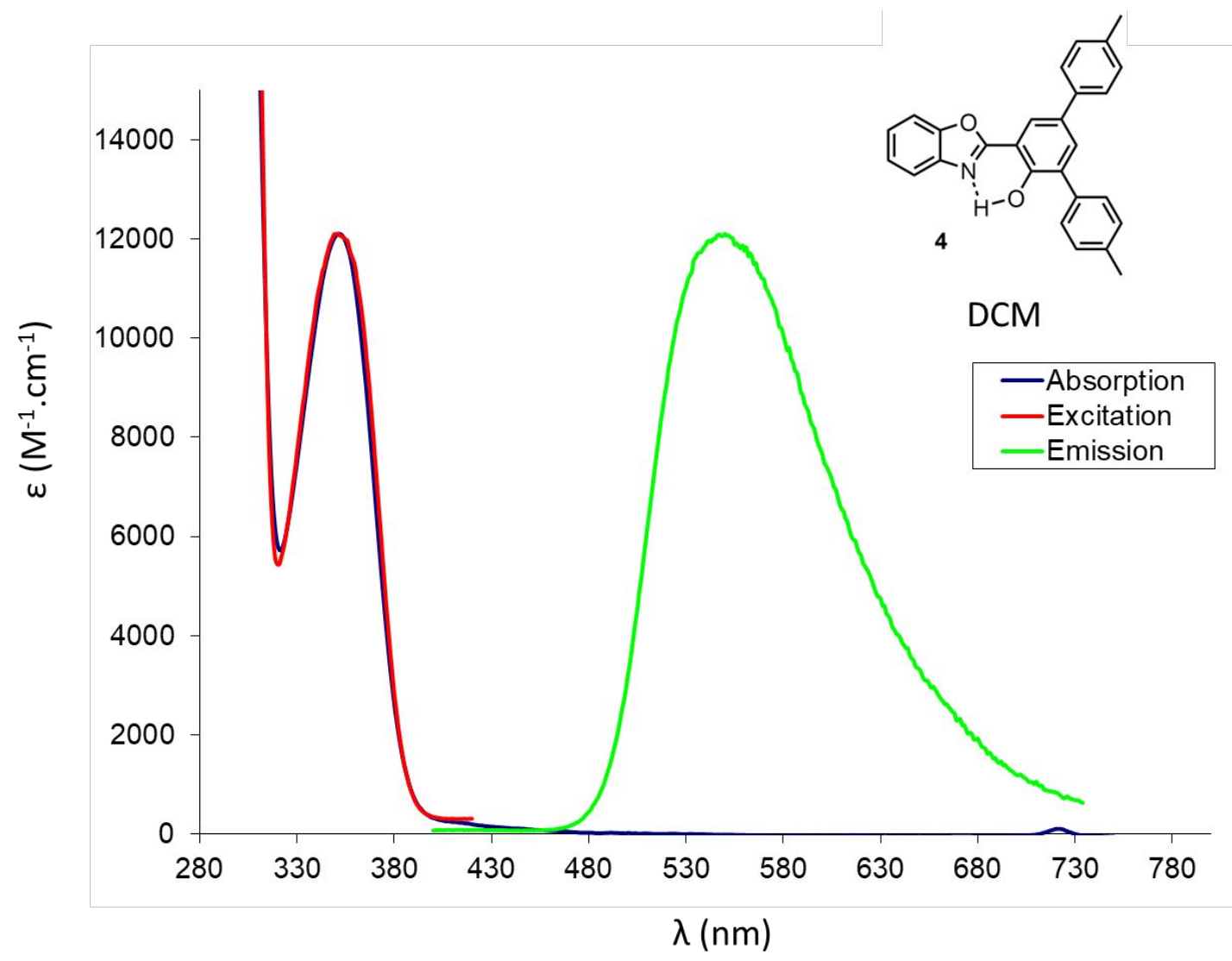

Figure S52. Absorption (blue), Emission (green) and Excitation (red) spectra of $\mathrm{HBO} 4$ in $\mathrm{CH}_{2} \mathrm{Cl}_{2}$. 


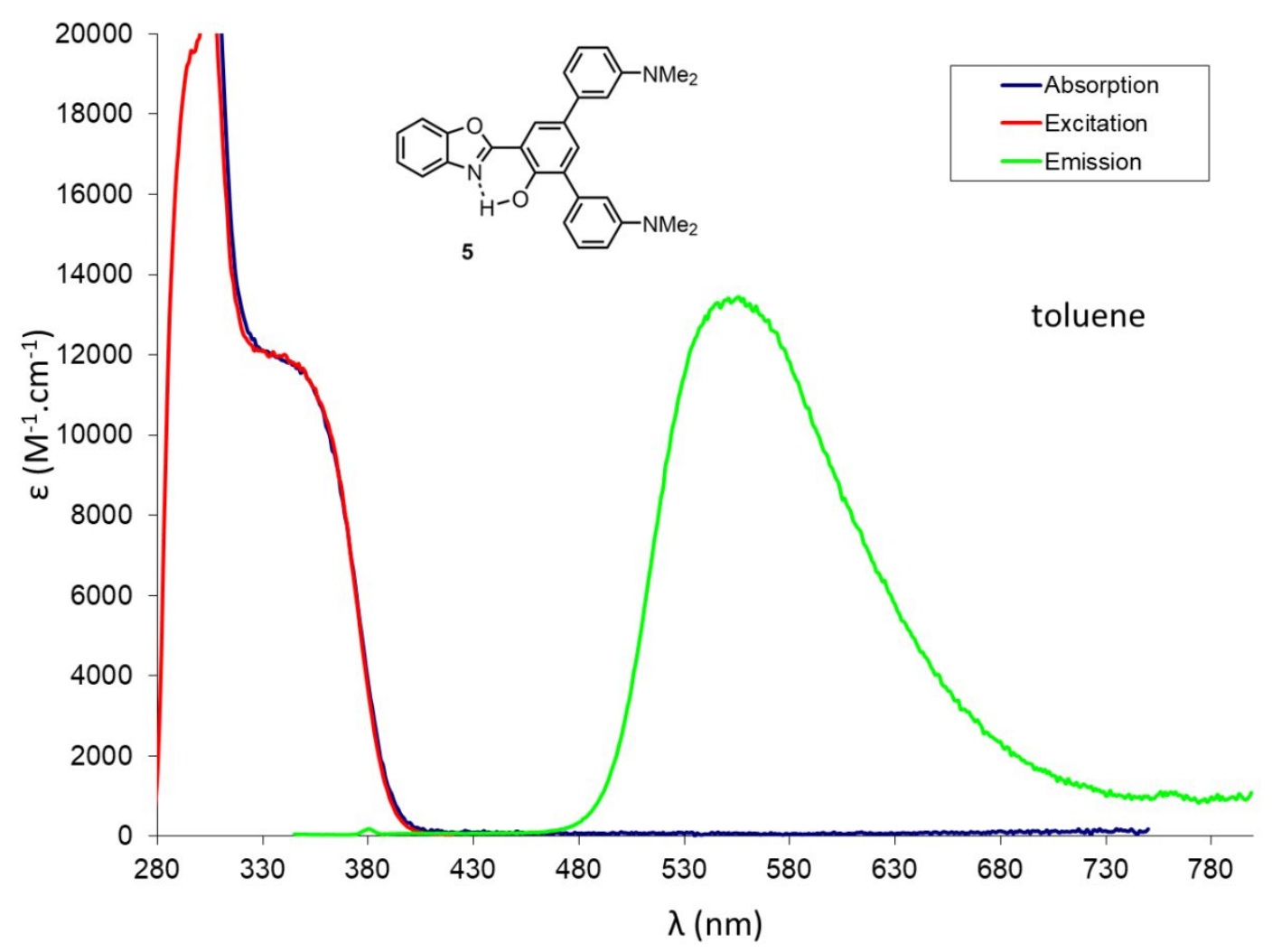

Figure S53. Absorption (blue), Emission (green) and Excitation (red) spectra of HBO 5 in toluene.

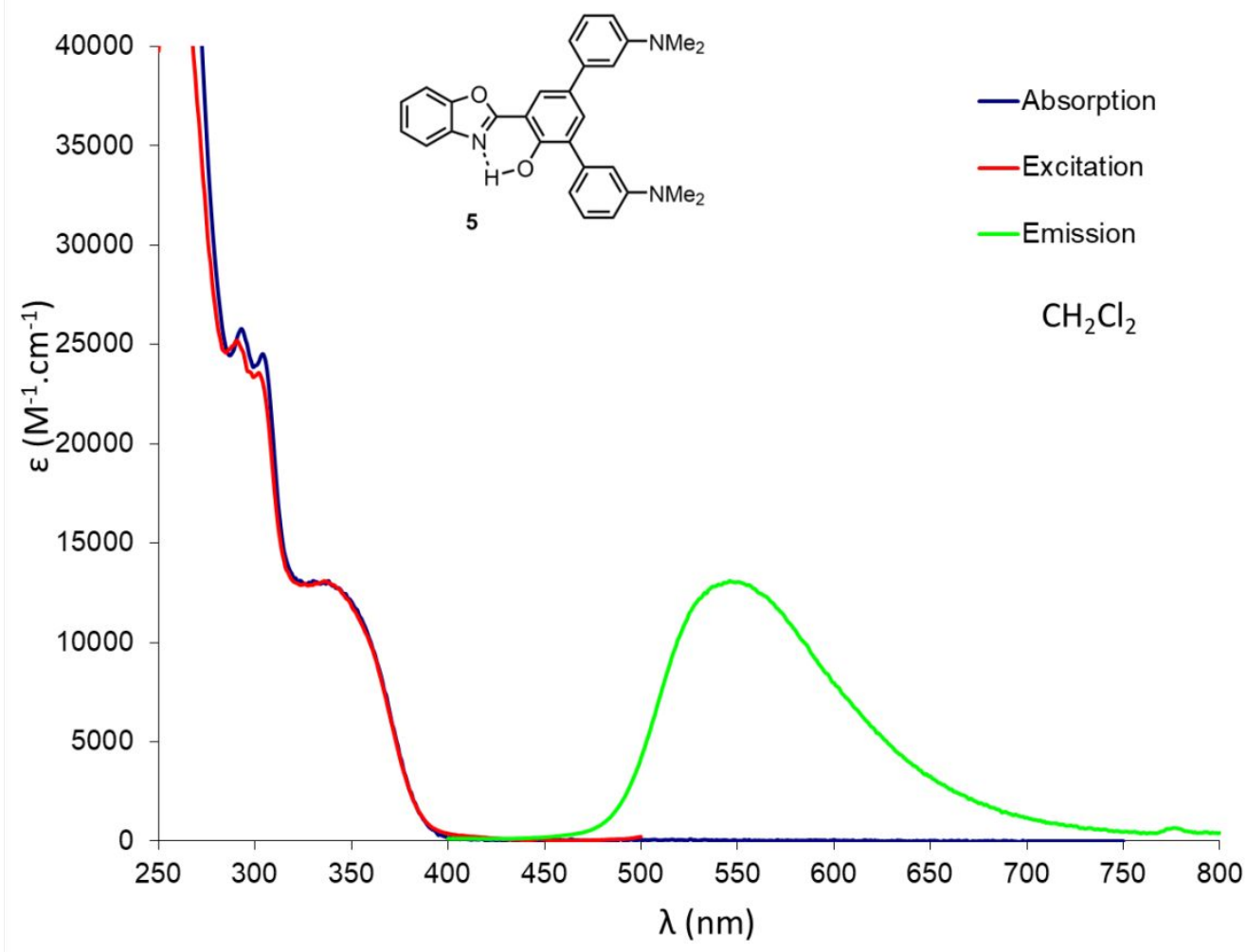

Figure S54. Absorption (blue), Emission (green) and Excitation (red) spectra of $\mathrm{HBO} 5$ in $\mathrm{CH}_{2} \mathrm{Cl}_{2}$. 


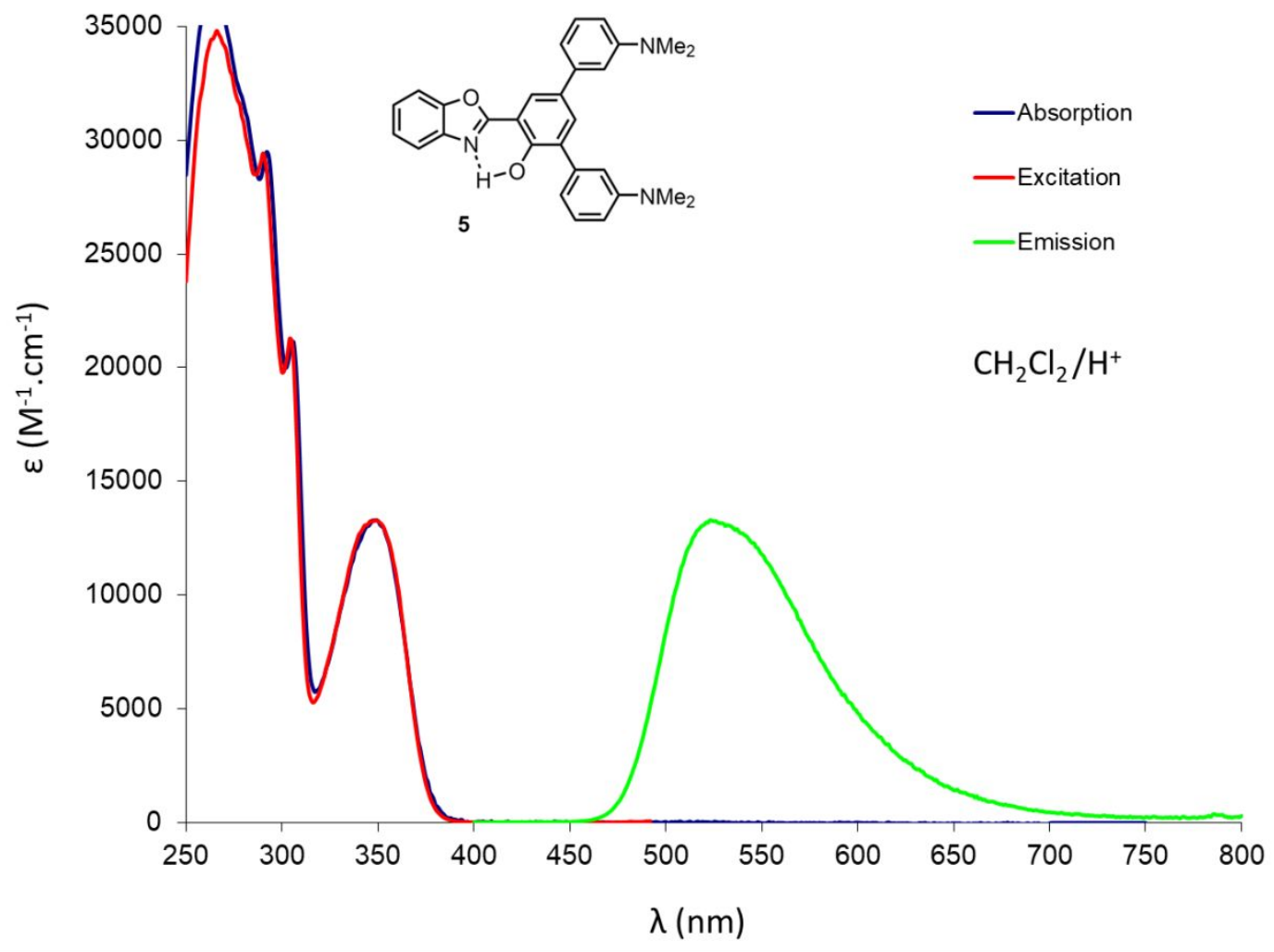

Figure S55. Absorption (blue), Emission (green) and Excitation (red) spectra of HBO 5 in protonated $\mathrm{CH}_{2} \mathrm{Cl}_{2}$.

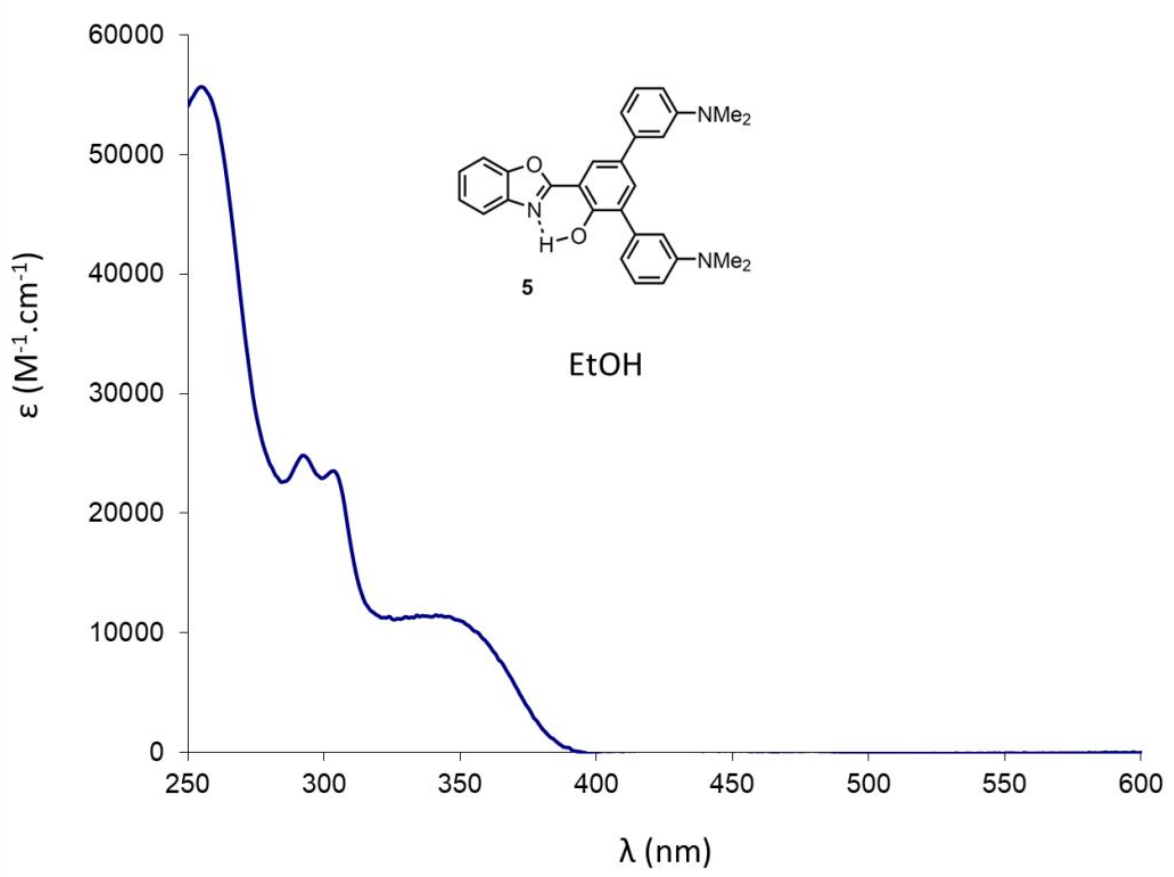

Figure S56. Absorption spectrum of $\mathrm{HBO} 5$ in EtOH. 


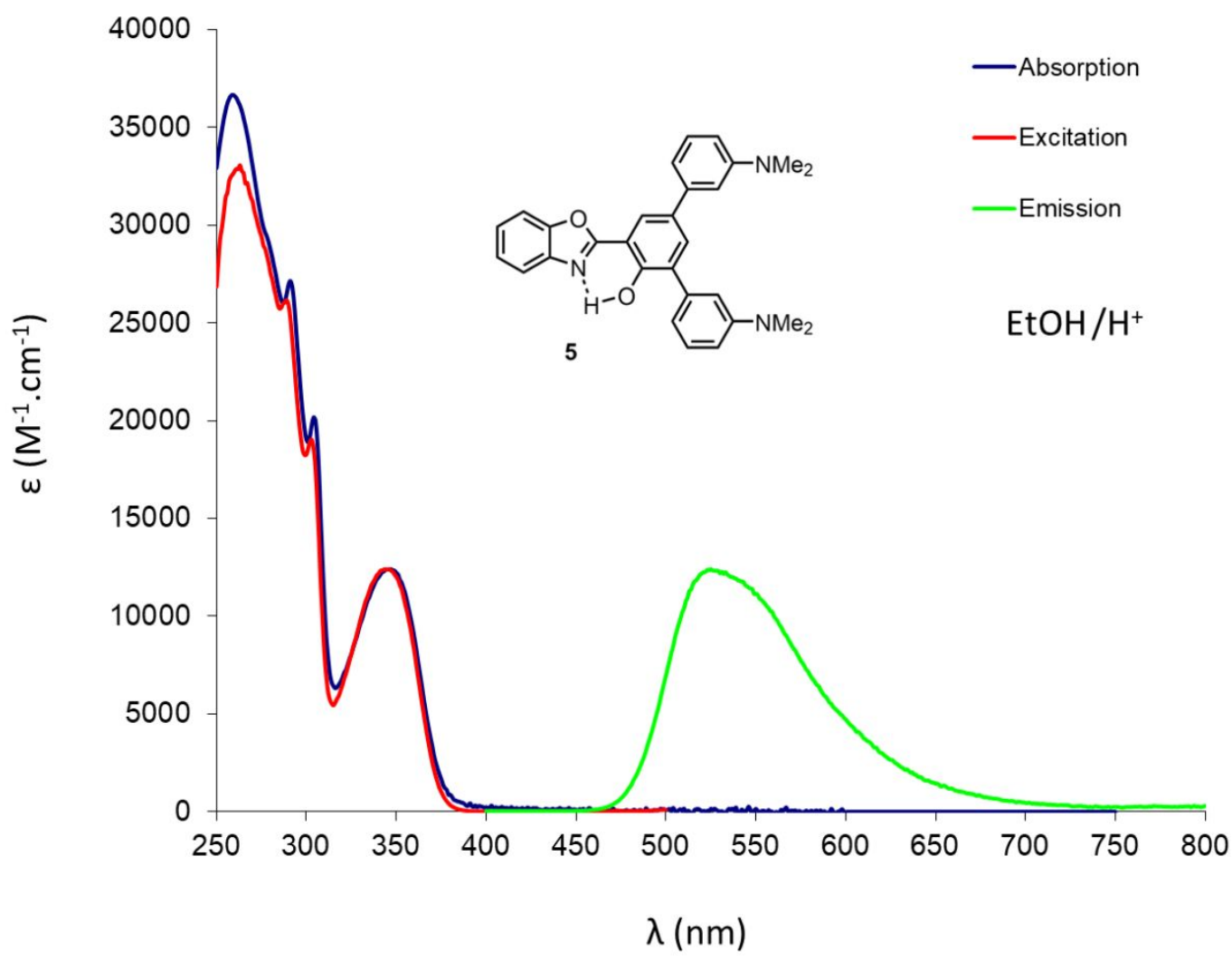

Figure S57. Absorption (blue), Emission (green) and Excitation (red) spectra of HBO 5 in protonated ethanol.

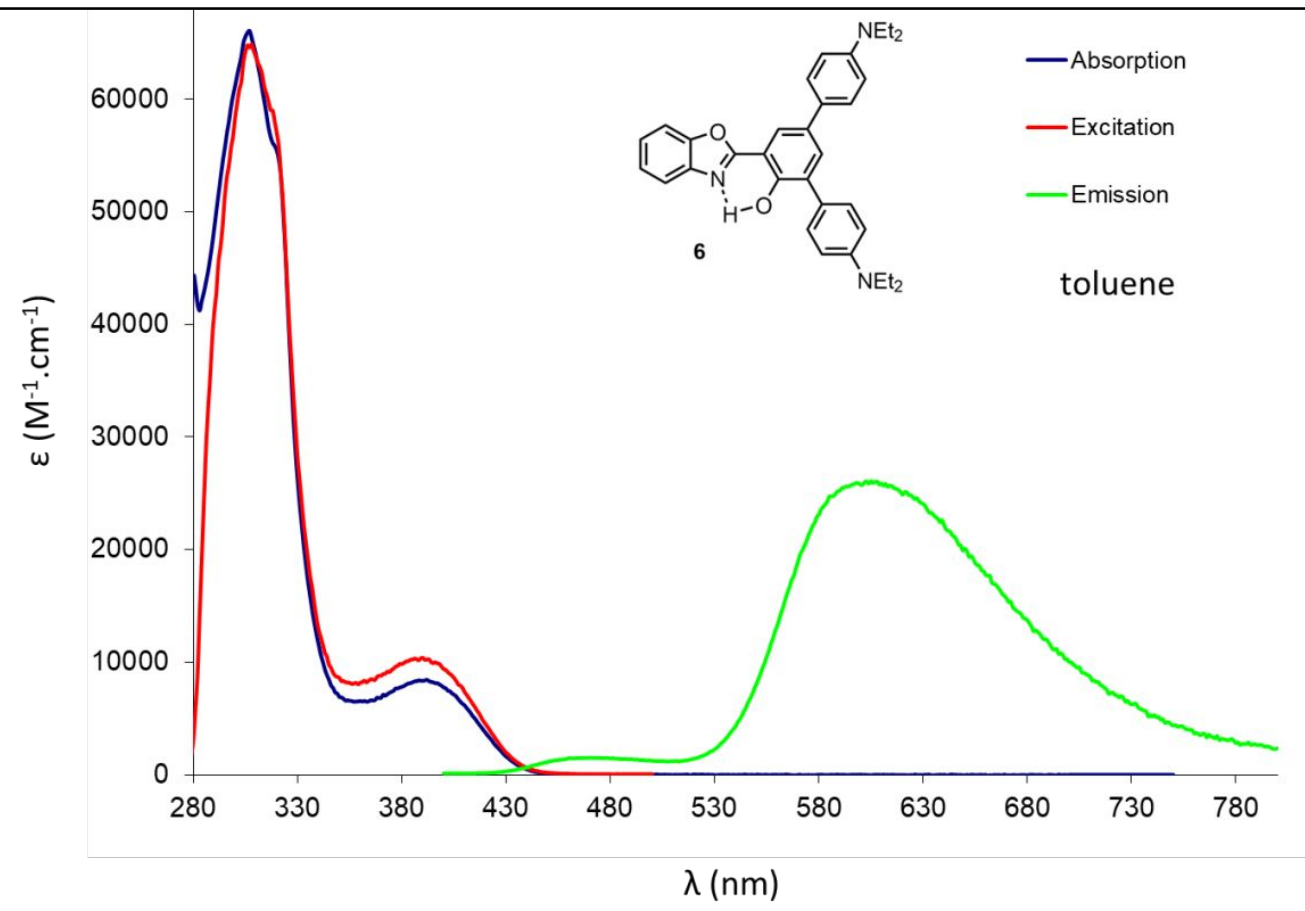

Figure S58. Absorption (blue), Emission (green) and Excitation (red) spectra of HBO 6 in toluene. 


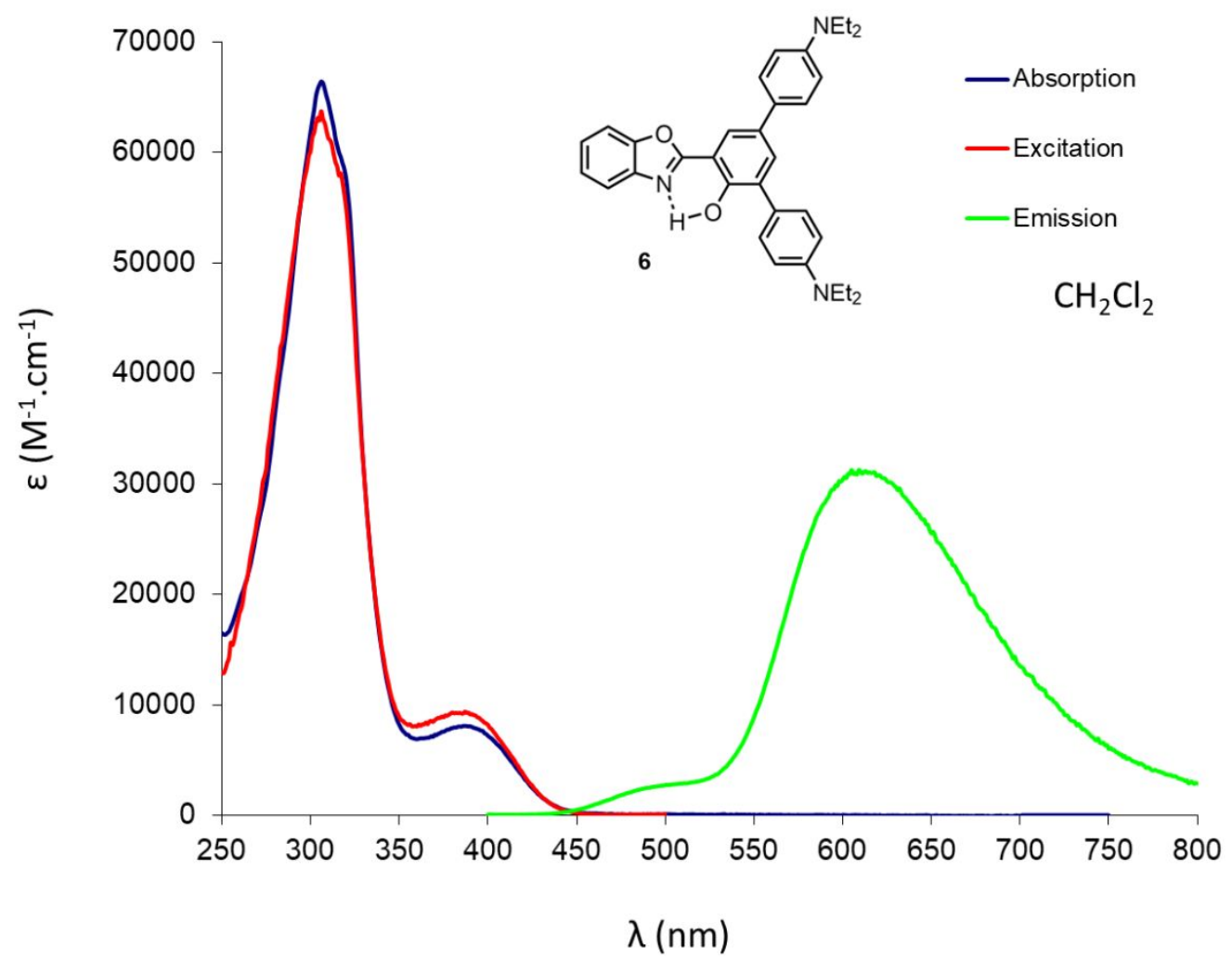

Figure S59. Absorption (blue), Emission (green) and Excitation (red) spectra of $\mathrm{HBO} 6$ in $\mathrm{CH}_{2} \mathrm{Cl}_{2}$.

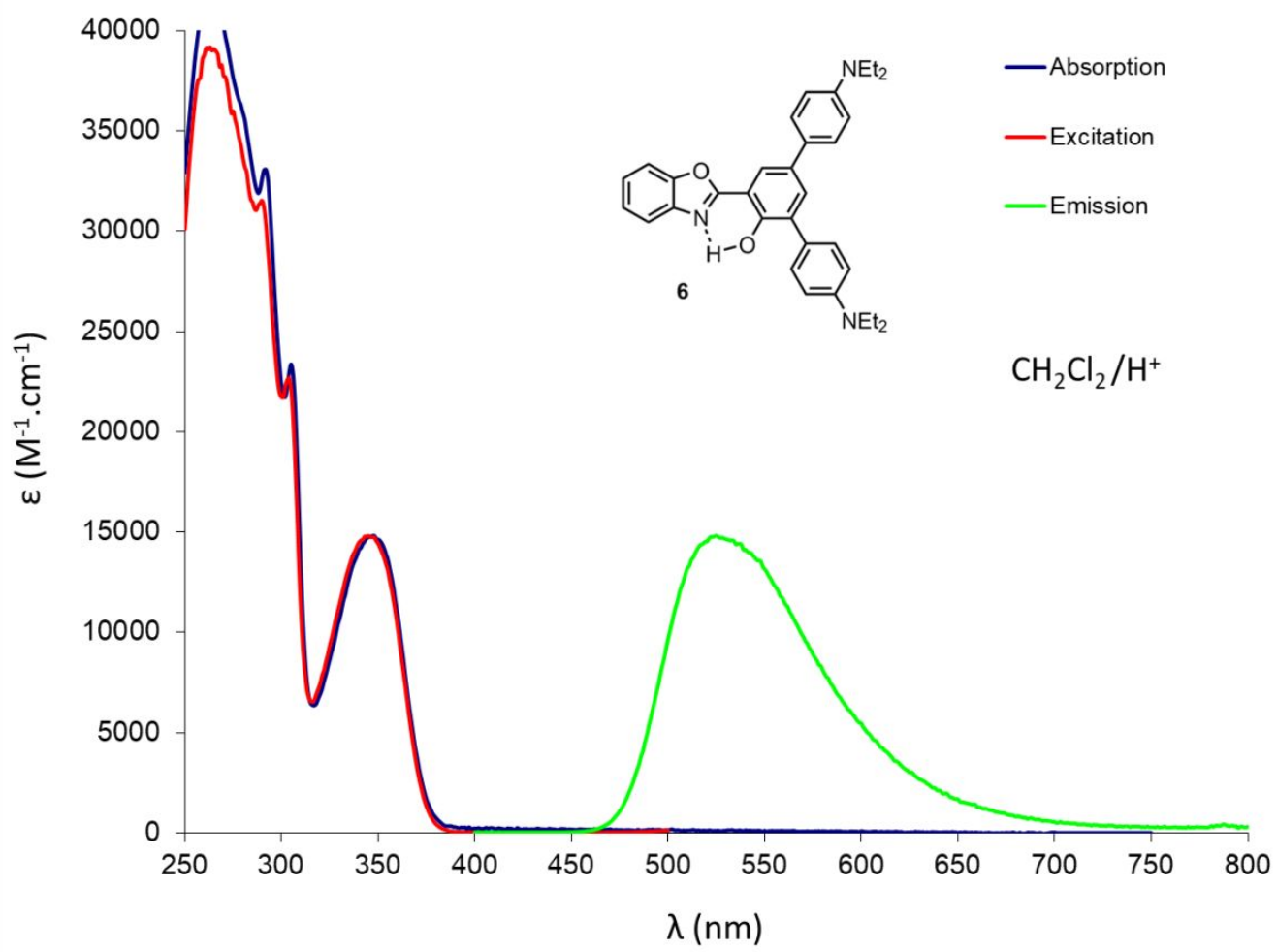

Figure S60. Absorption (blue), Emission (green) and Excitation (red) spectra of $\mathrm{HBO} 6$ in protonated $\mathrm{CH}_{2} \mathrm{Cl}_{2}$. 


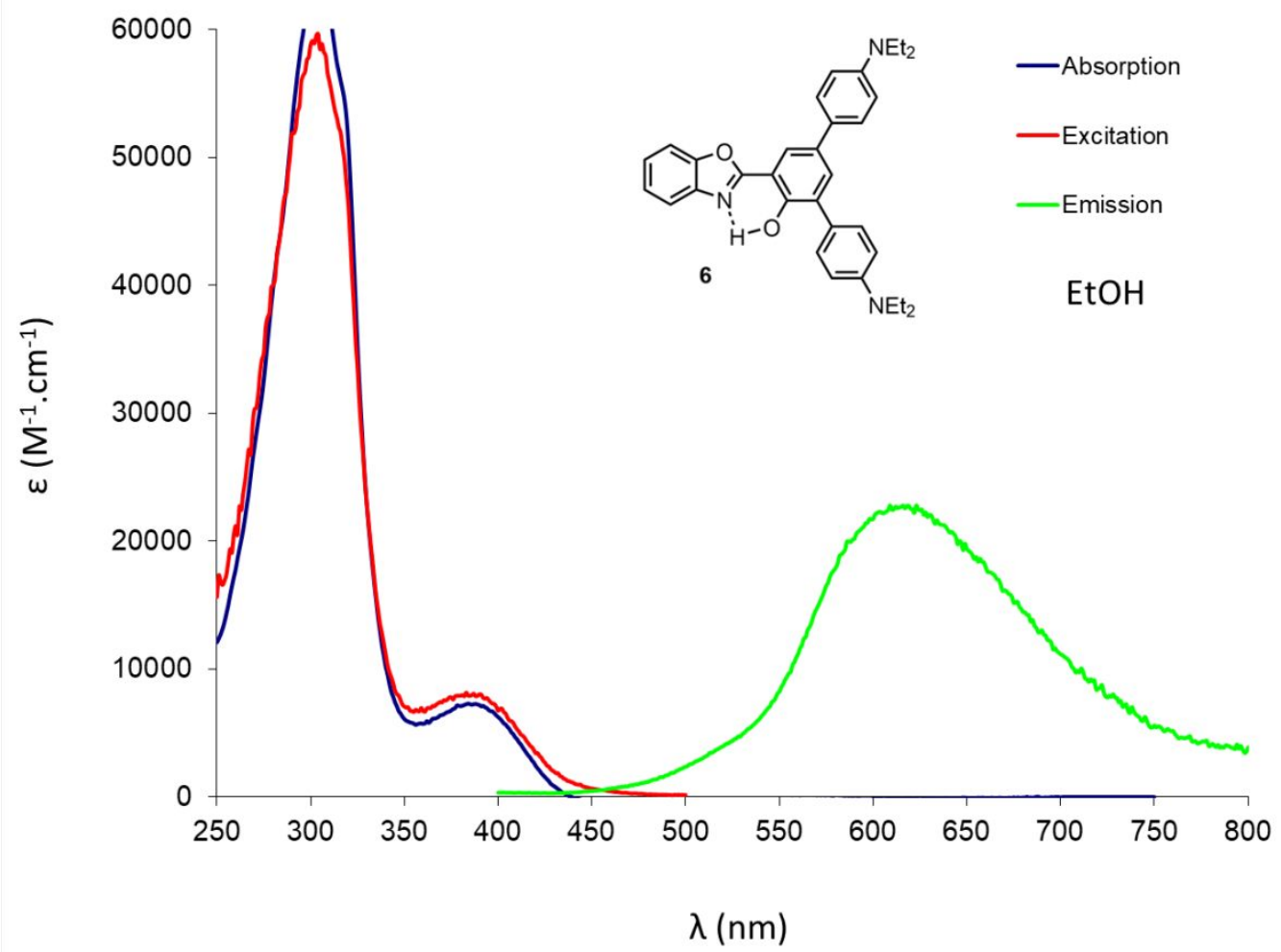

Figure S61. Absorption (blue), Emission (green) and Excitation (red) spectra of HBO 6 in EtOH.

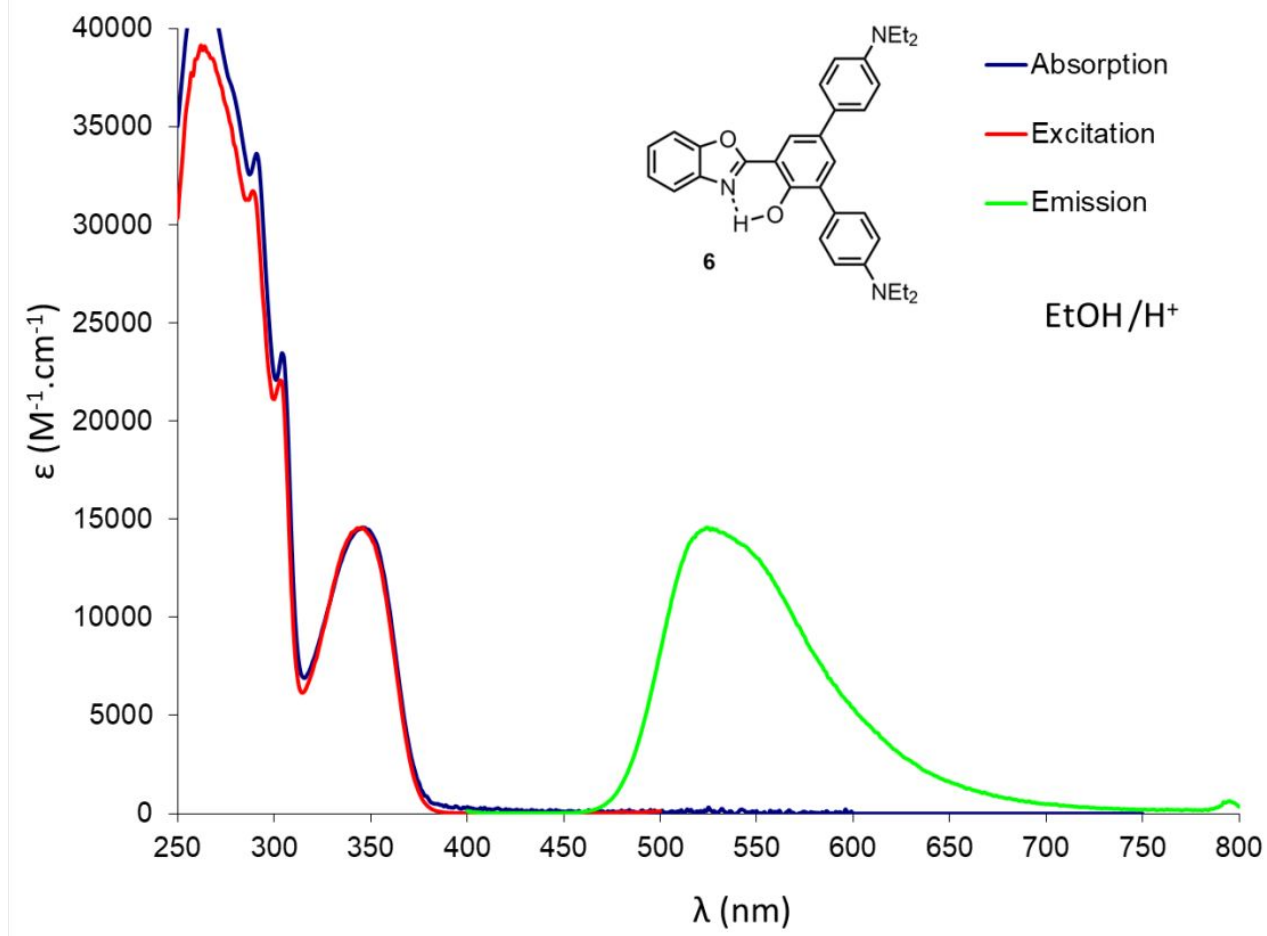

Figure S62. Absorption (blue), Emission (green) and Excitation (red) spectra of HBO 6 in protonated ethanol. 


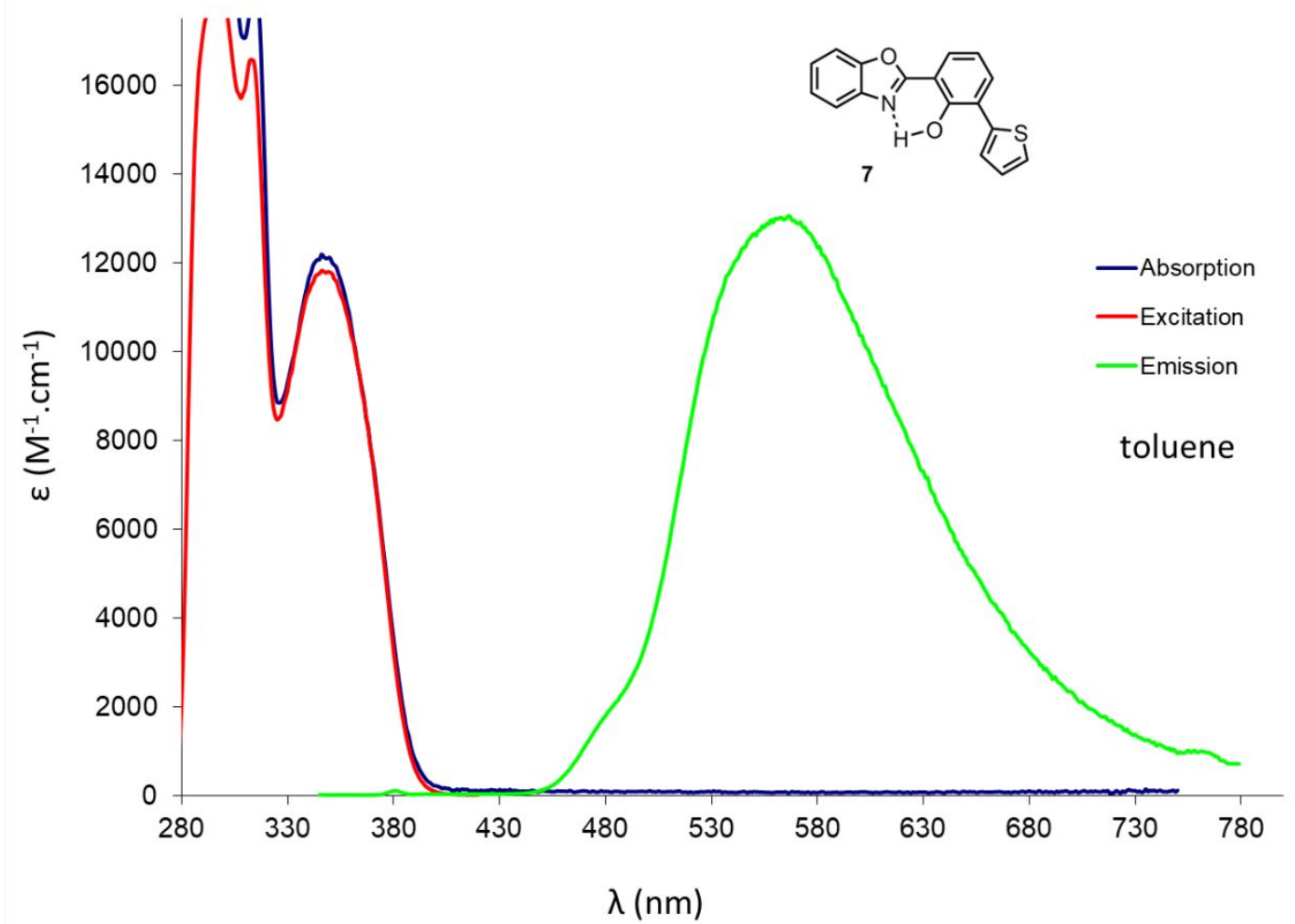

Figure S63. Absorption (blue), Emission (green) and Excitation (red) spectra of $\mathrm{HBO} 7$ in toluene.

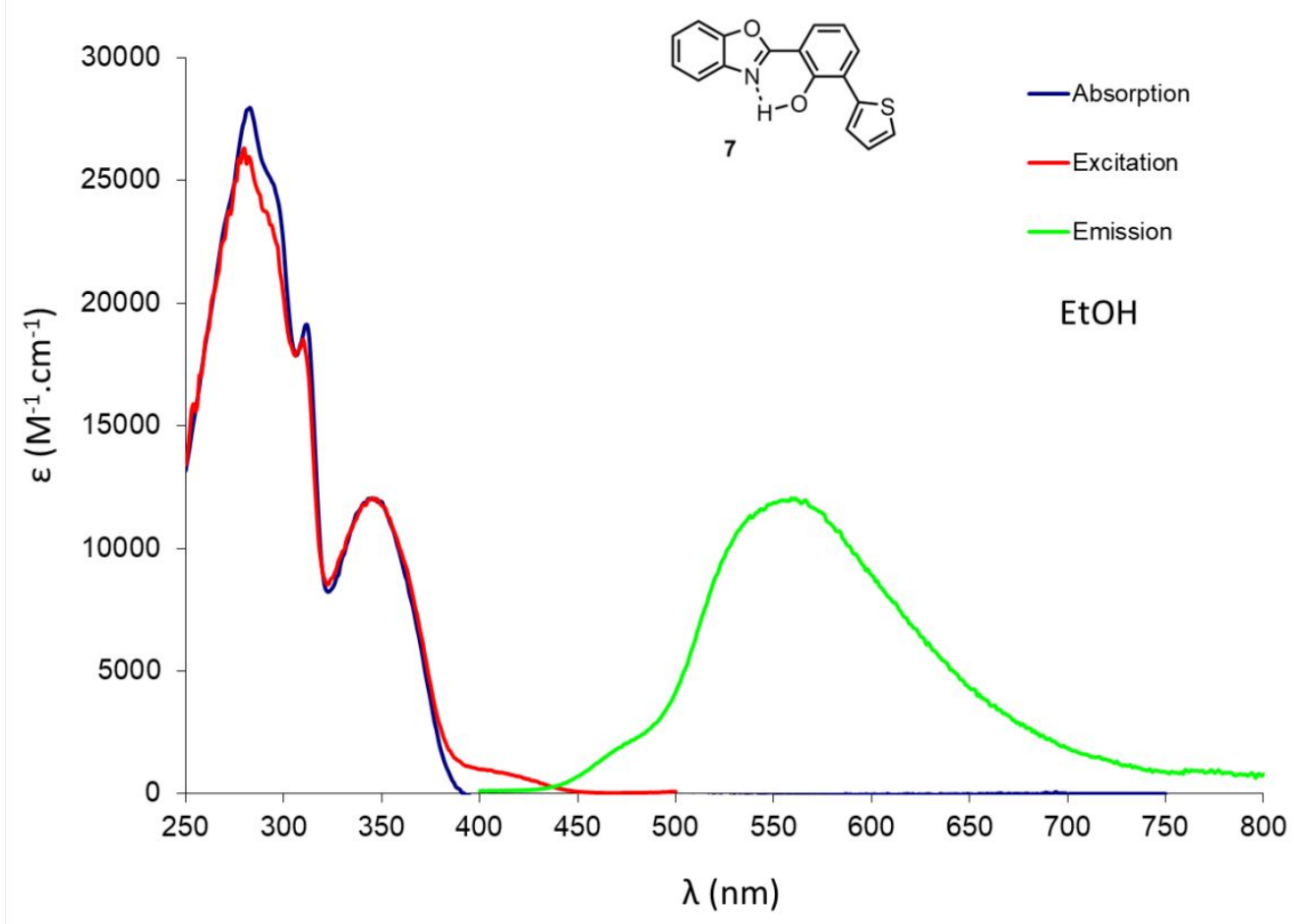

Figure S64. Absorption (blue), Emission (green) and Excitation (red) spectra of $\mathrm{HBO} 7$ in EtOH. 


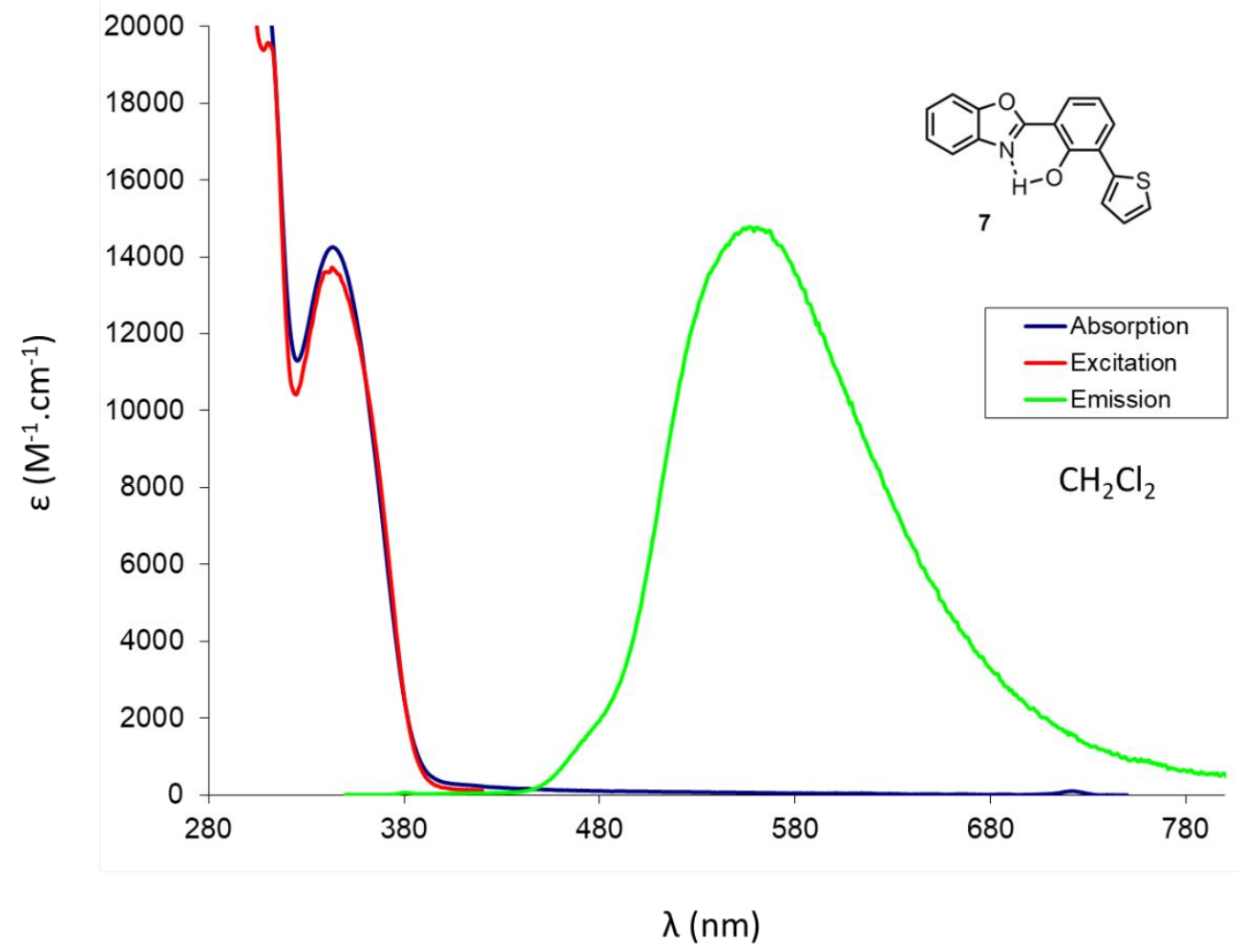

Figure S65. Absorption (blue), Emission (green) and Excitation (red) spectra of $\mathrm{HBO} 7$ in $\mathrm{CH}_{2} \mathrm{Cl}_{2}$.

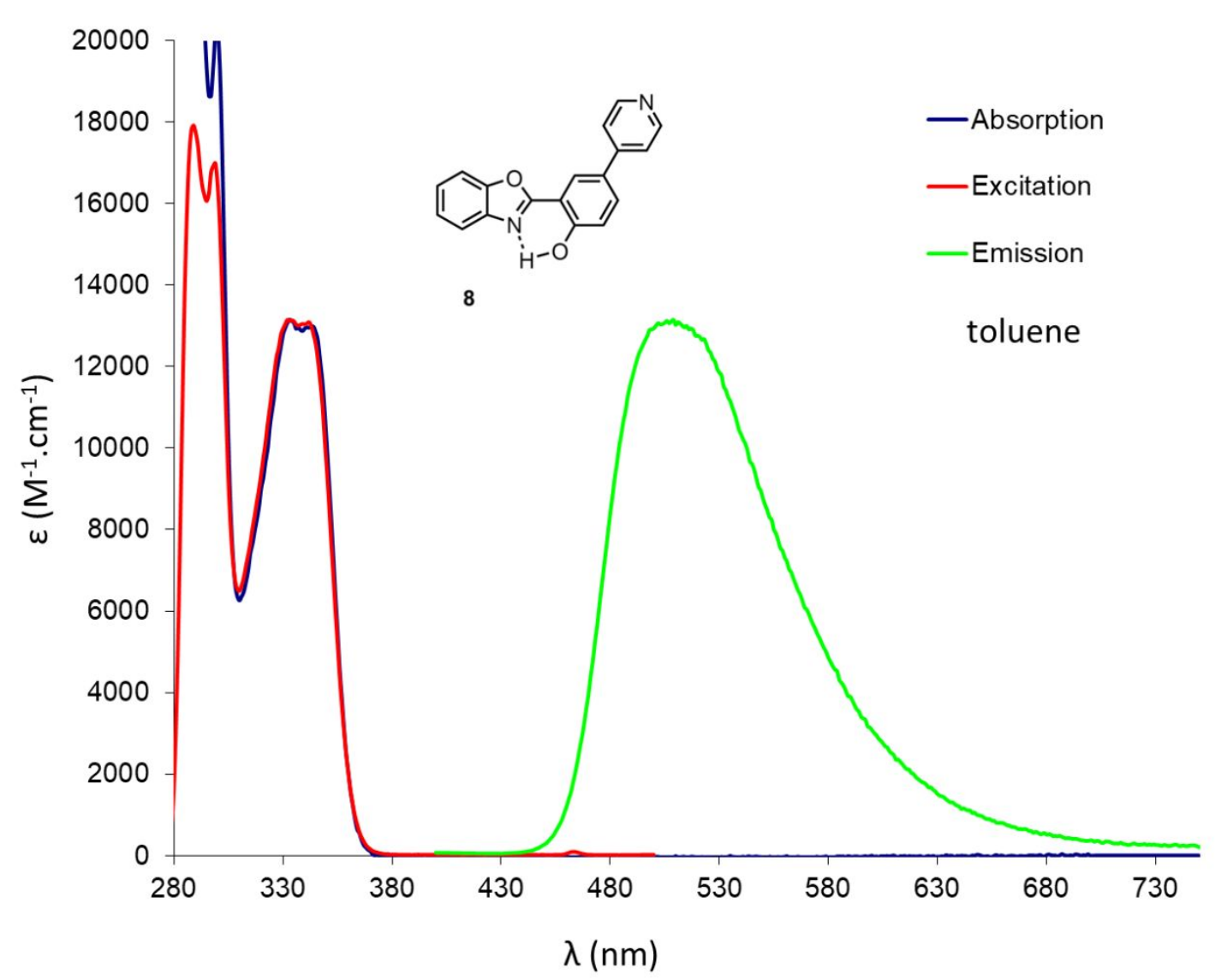

Figure S66. Absorption (blue), Emission (green) and Excitation (red) spectra of HBO 8 in toluene. 


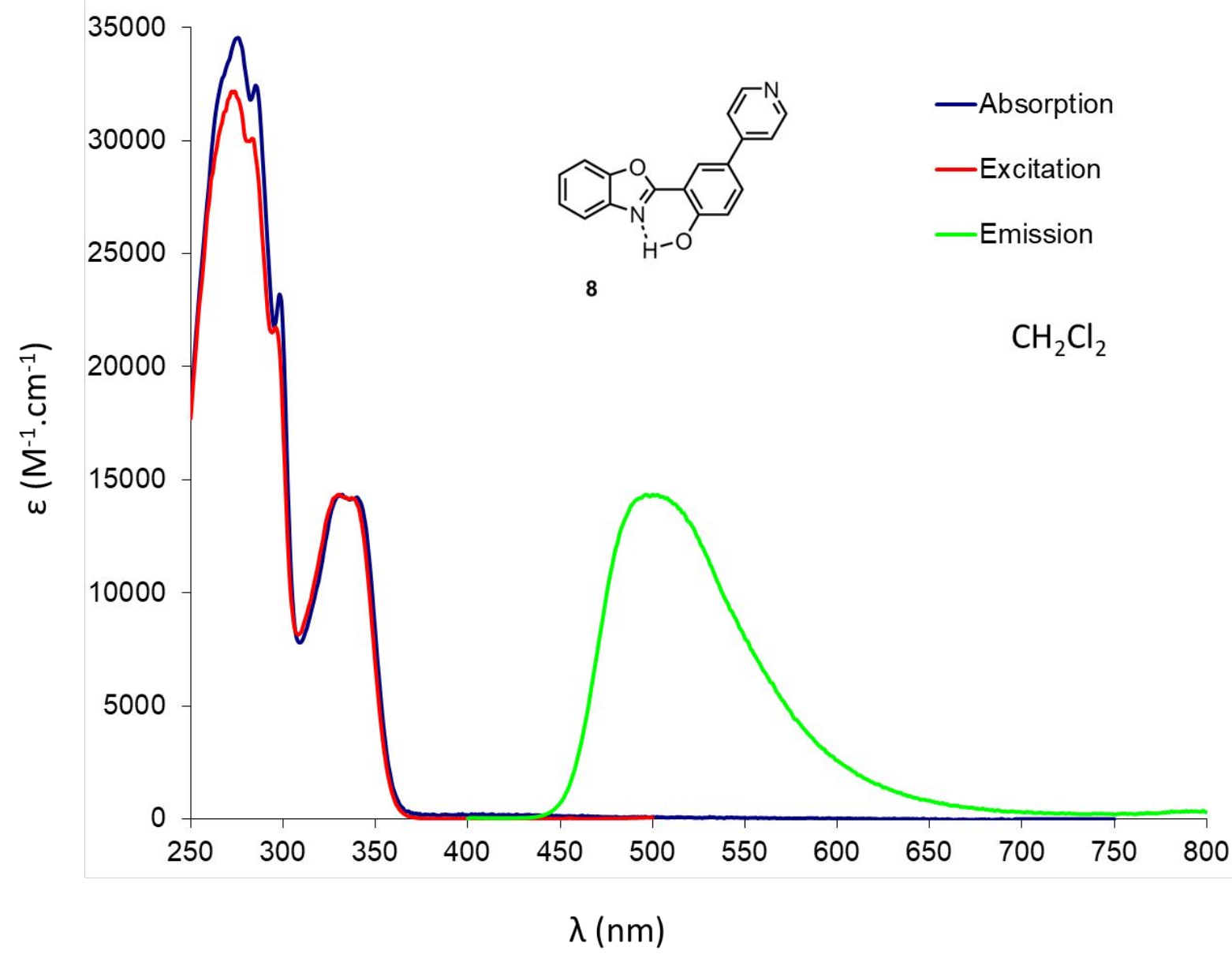

Figure S67. Absorption (blue), Emission (green) and Excitation (red) spectra of $\mathrm{HBO} 8$ in $\mathrm{CH}_{2} \mathrm{Cl}_{2}$.

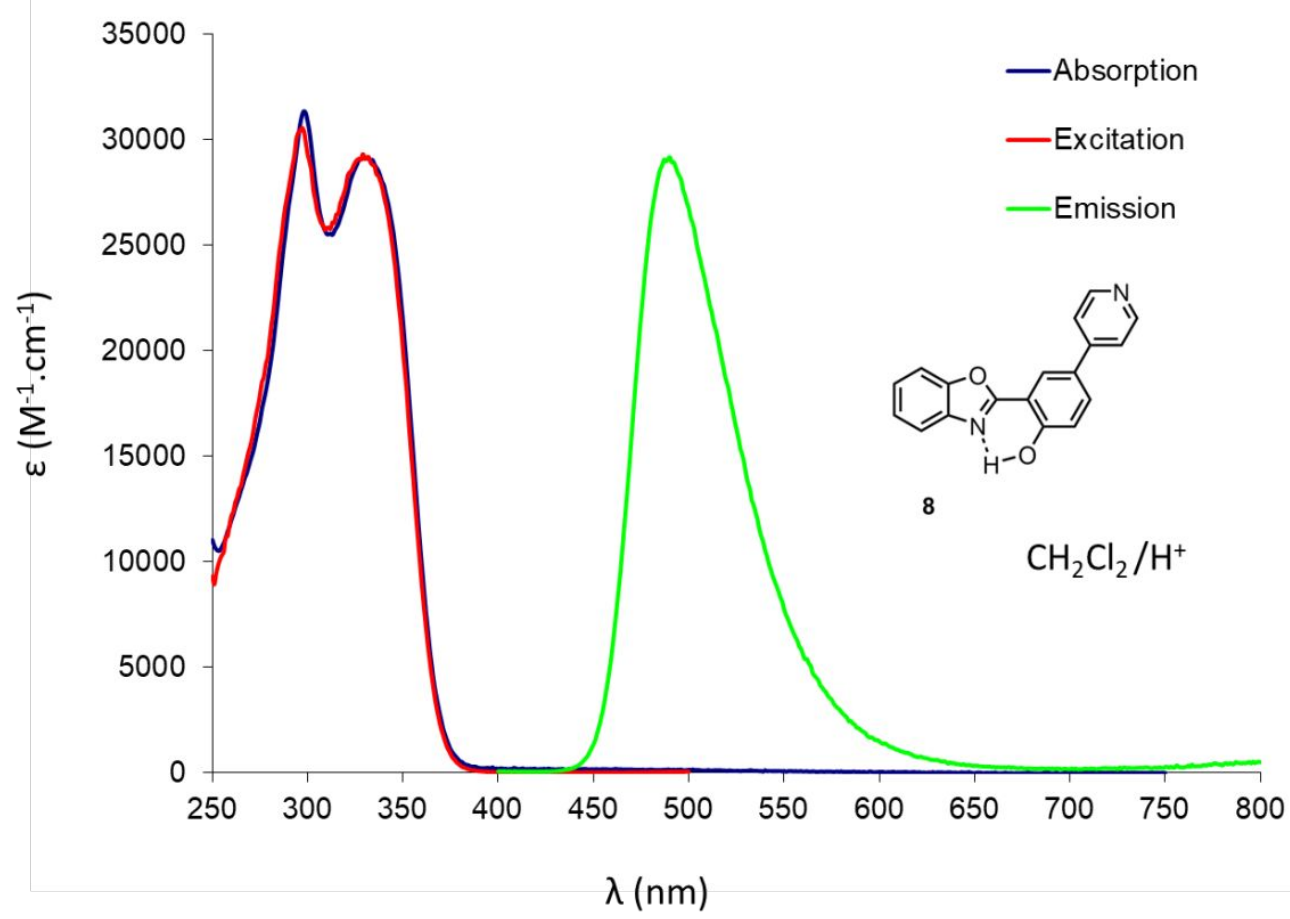

Figure S68. Absorption (blue), Emission (green) and Excitation (red) spectra of $\mathrm{HBO} 8$ in protonated $\mathrm{CH}_{2} \mathrm{Cl}_{2}$. 


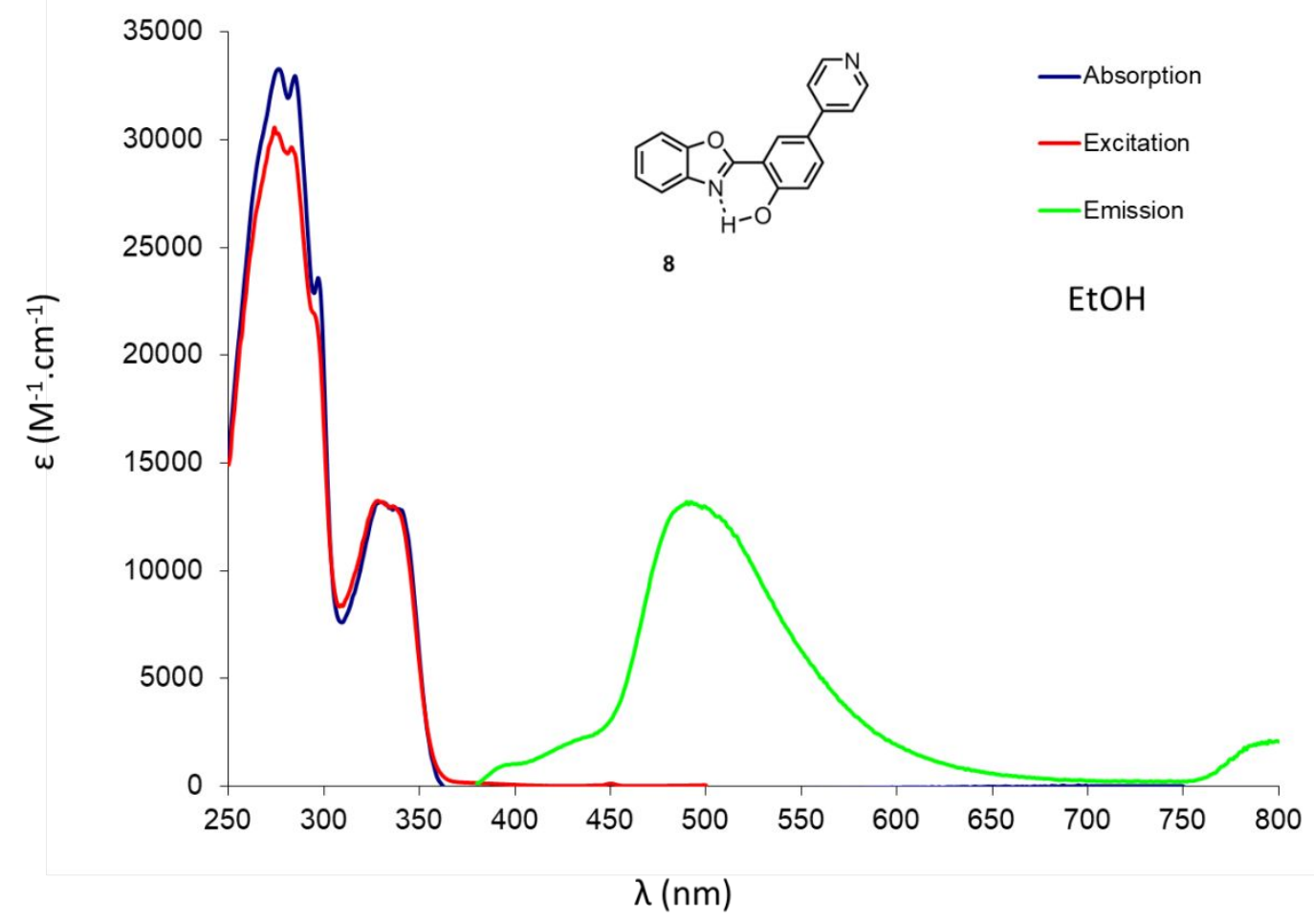

Figure S69. Absorption (blue), Emission (green) and Excitation (red) spectra of HBO 8 in EtOH.

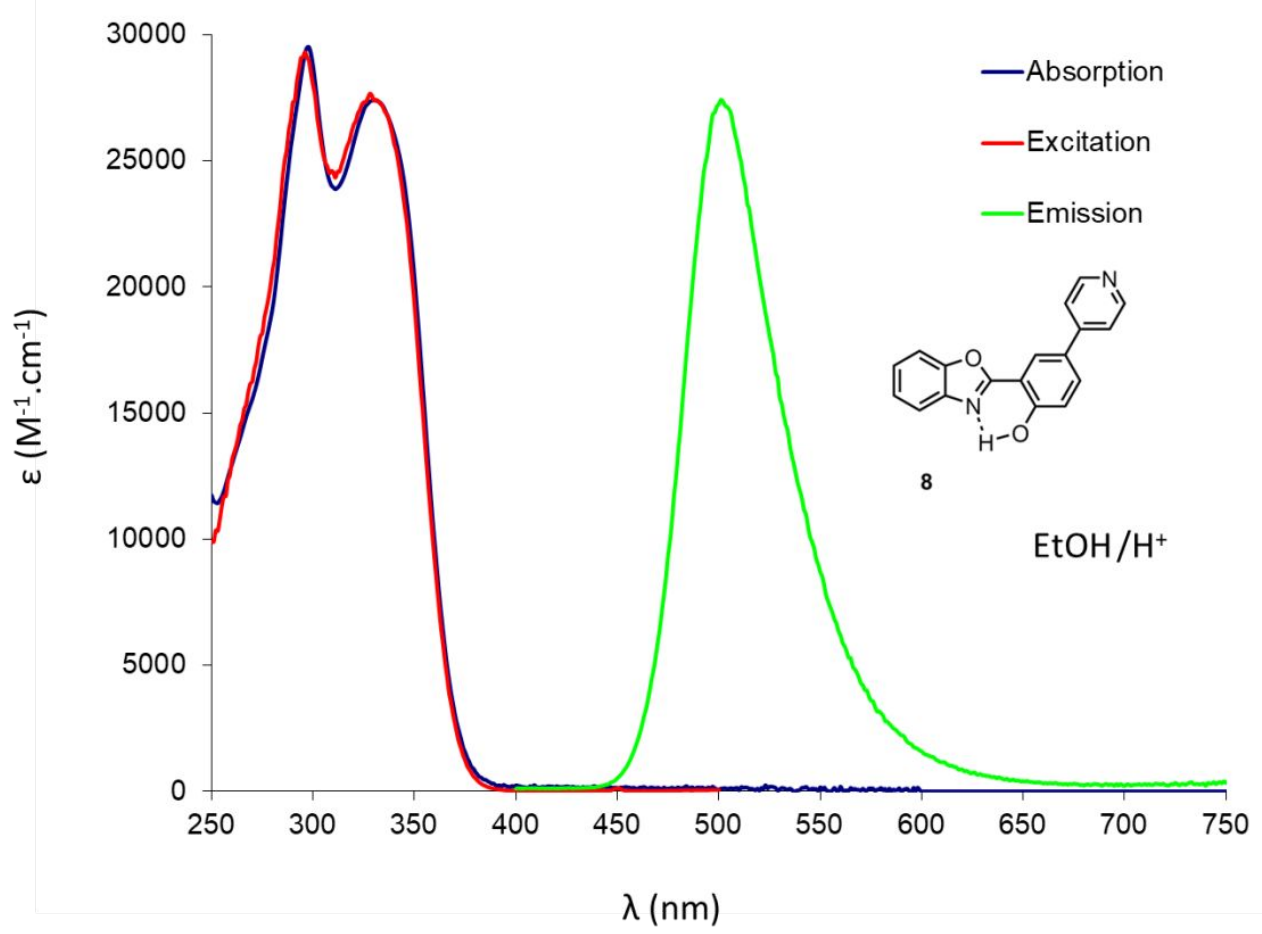

Figure S70. Absorption (blue), Emission (green) and Excitation (red) spectra of HBO 8 in protonated EtOH. 
Table S1. Photophysical data in aerated solutions in ethanol at $25^{\circ} \mathrm{C}$.

\begin{tabular}{|c|c|c|c|c|c|c|c|c|c|}
\hline HBO & $\begin{array}{c}\lambda_{\mathrm{abs}} \\
(\mathrm{nm})\end{array}$ & $\begin{array}{c}\varepsilon \\
\left(M^{-1} . \mathrm{cm}^{-1}\right)\end{array}$ & $\begin{array}{c}\lambda_{\mathrm{em}} \\
(\mathrm{nm})\end{array}$ & $\Delta_{\mathrm{ss}}^{[\mathrm{b}]}$ & $\Phi_{\mathrm{f}}{ }^{[a]}$ & $\begin{array}{c}T \\
\text { (ns) }\end{array}$ & $\mathrm{K}_{\mathrm{r}}[\mathrm{c}]$ & $\mathrm{K}_{\mathrm{nr}}[\mathrm{c}]$ & Solvent \\
\hline 1 & 329 & 17200 & [d] & [d] & [d] & [d] & [d] & [d] & $\mathrm{EtOH}$ \\
\hline 1 & 329 & 18800 & 517 & 11100 & 0.15 & 1.7 & 0.9 & 5.0 & $\mathrm{EtOH} / \mathrm{H}^{+}$ \\
\hline 2 & 352 & 9500 & [d] & [d] & [d] & [d] & [d] & [d] & $\mathrm{EtOH}$ \\
\hline 2 & 331 & 18000 & 518 & 10900 & 0.13 & 0.2 & 6.5 & 43.5 & $\mathrm{EtOH} / \mathrm{H}^{+}$ \\
\hline 3 & 363 & 2600 & [d] & [d] & [d] & [d] & [d] & [d] & $\mathrm{EtOH}$ \\
\hline 3 & 336 & 9500 & 507 & 10000 & 0.18 & 0.1 & 18.0 & 82.0 & $\mathrm{EtOH} / \mathrm{H}^{+}$ \\
\hline 4 & 352 & 11200 & 544 & 10000 & 0.03 & 0.6 & 0.5 & 16.2 & $\mathrm{EtOH}$ \\
\hline 5 & 343 & 11500 & [d] & [d] & [d] & [d] & [d] & [d] & $\mathrm{EtOH}$ \\
\hline 5 & 345 & 12400 & 525 & 9900 & 0.18 & 2.8 & 0.6 & 2.9 & $\mathrm{EtOH} / \mathrm{H}^{+}$ \\
\hline 6 & 388 & 7900 & [d] & [d] & [d] & [d] & [d] & [d] & $\mathrm{EtOH}$ \\
\hline 6 & 347 & 14500 & 524 & 9700 & 0.16 & 3.0 & 0.2 & 1.1 & $\mathrm{EtOH} / \mathrm{H}^{+}$ \\
\hline 7 & 345 & 12000 & 561 & 11200 & 0.03 & 2.9 & 0.1 & 3.3 & $\mathrm{EtOH}$ \\
\hline 8 & 330 & 13200 & $430 / 492$ & 7000 & 0.06 & 1.4 & 0.4 & 6.6 & $\mathrm{EtOH}$ \\
\hline 8 & 329 & 27600 & 501 & 10400 & 0.06 & 0.9 & 0.7 & 1.0 & $\mathrm{EtOH} / \mathrm{H}^{+}$ \\
\hline
\end{tabular}

[a] Relative quantum yield determined in solution using Rhodamine $6 \mathrm{G}$ as a reference $\left(\lambda_{\text {exc }}=488 \mathrm{~nm}, \Phi=0.88\right.$ in ethanol), [b] Stokes shift, ${ }^{[c]} k_{r}\left(10^{8} s^{-1}\right)$ and $k_{n r}\left(10^{8} s^{-1}\right)$ were calculated using: $k_{r}=\Phi_{F} / \sigma, k_{n r}=\left(1-\Phi_{F}\right) / \sigma$ where $\sigma$ is the lifetime. 


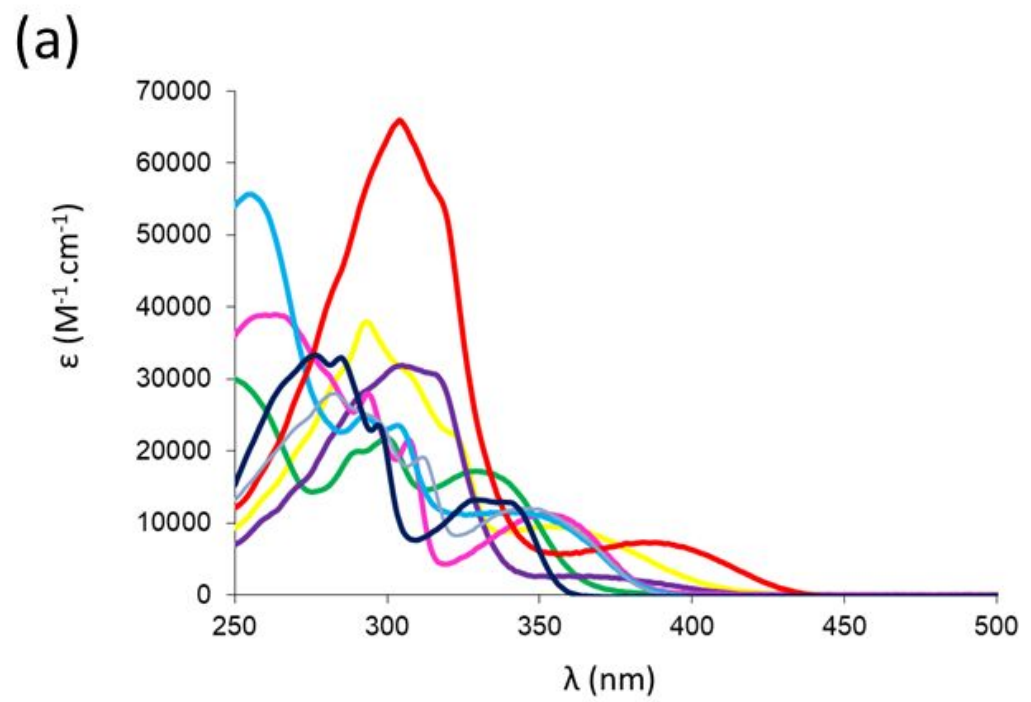

(b)

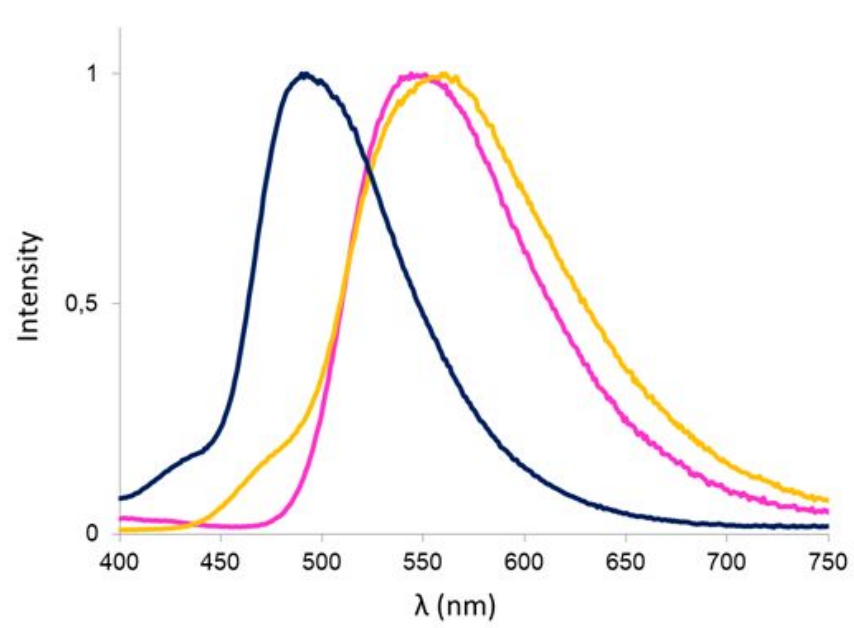

Figure S71. (a) Absorption and (b) emission spectra of HBO 1 (green), HBO 2 (yellow), HBO 3 (purple), HBO 4 (pink), HBO 5 (blue), HBO 6 (red), HBO 7 (orange) and HBO 8 (navy blue) in ethanol at $25^{\circ} \mathrm{C}$. 


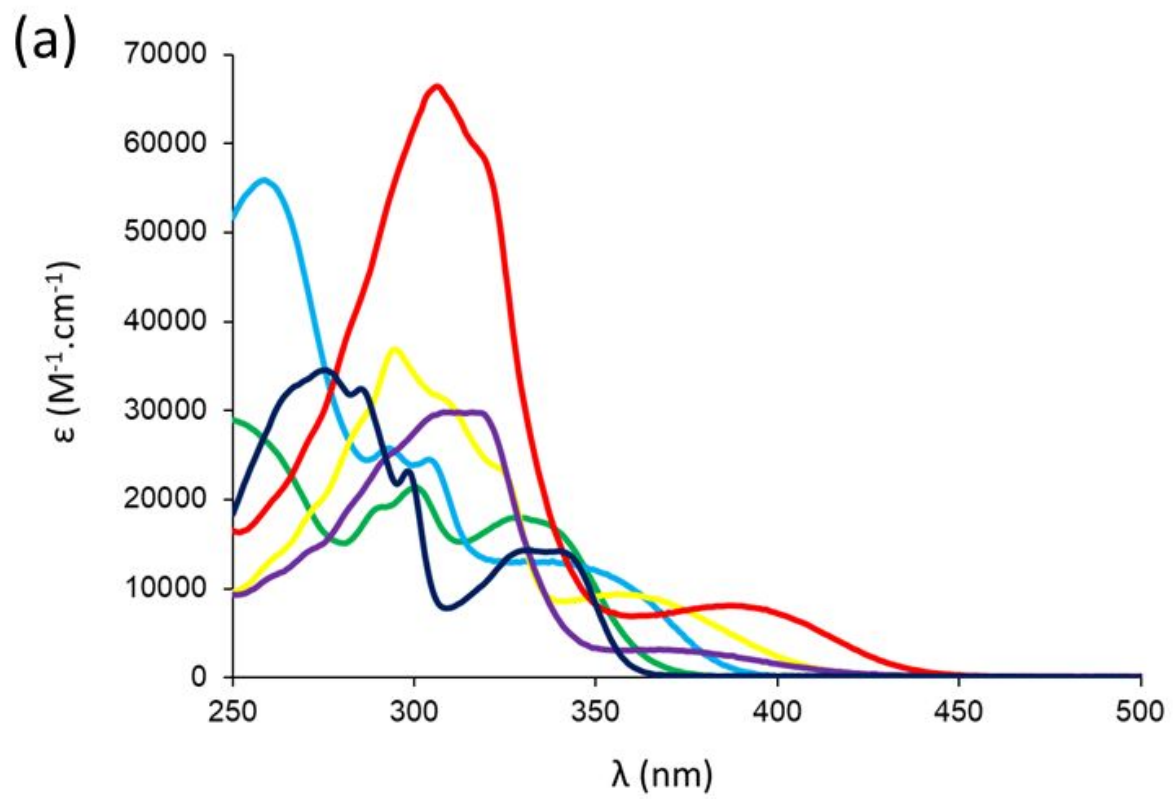

(b)

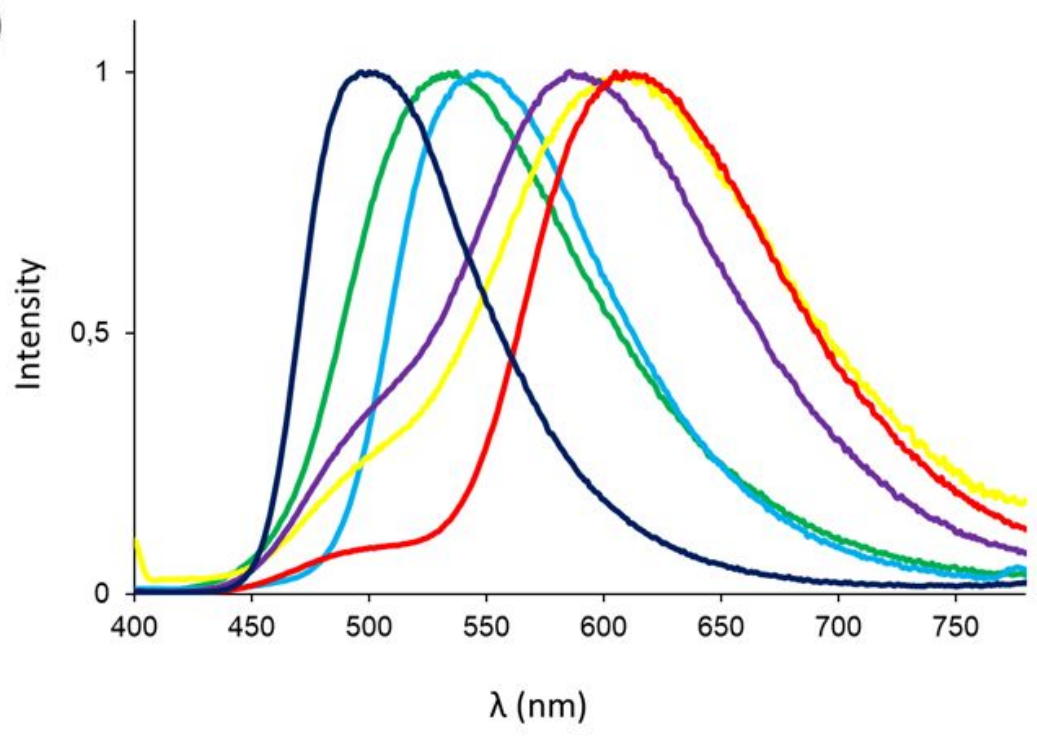

Figure S72. (a) Absorption and (b) emission spectra of HBO 1 (green), HBO 2 (yellow), HBO 3 (purple), HBO 5 (blue), HBO 6 (red) and HBO 8 (navy blue) in dichloromethane at $25^{\circ} \mathrm{C}$. 


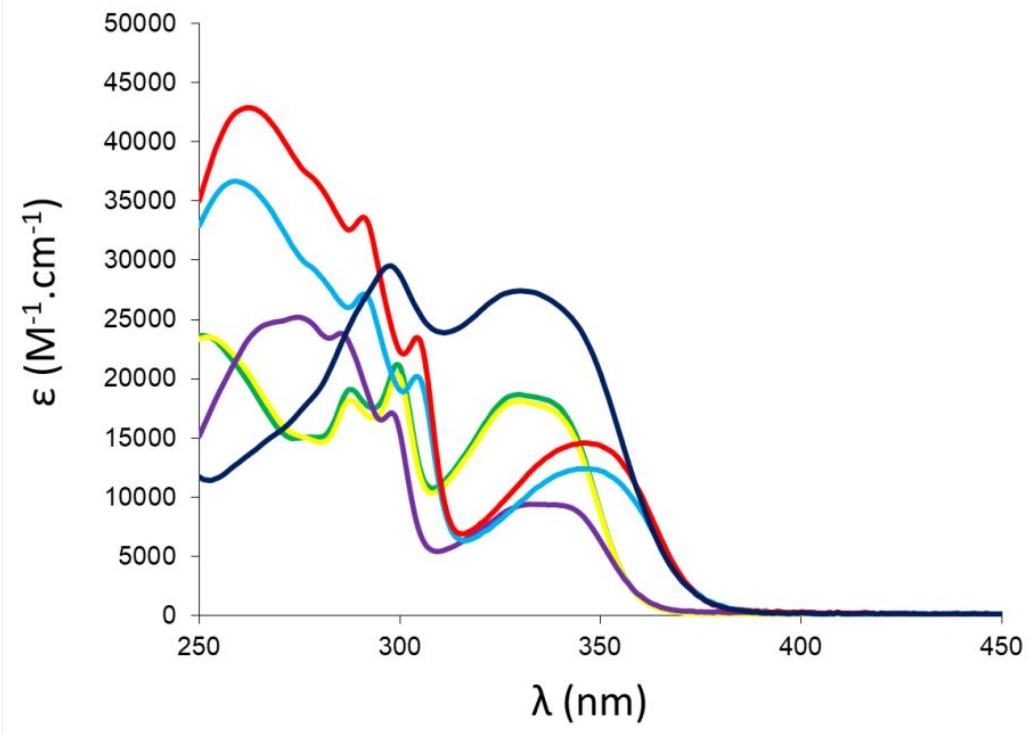

Figure S73. Absorption spectra of HBO 1 (green), HBO 2 (yellow), HBO 3 (purple), HBO 5 (blue), HBO 6 (red), HBO 8 (navy blue) in their protonated state in dichloromethane at $25^{\circ} \mathrm{C}$. 


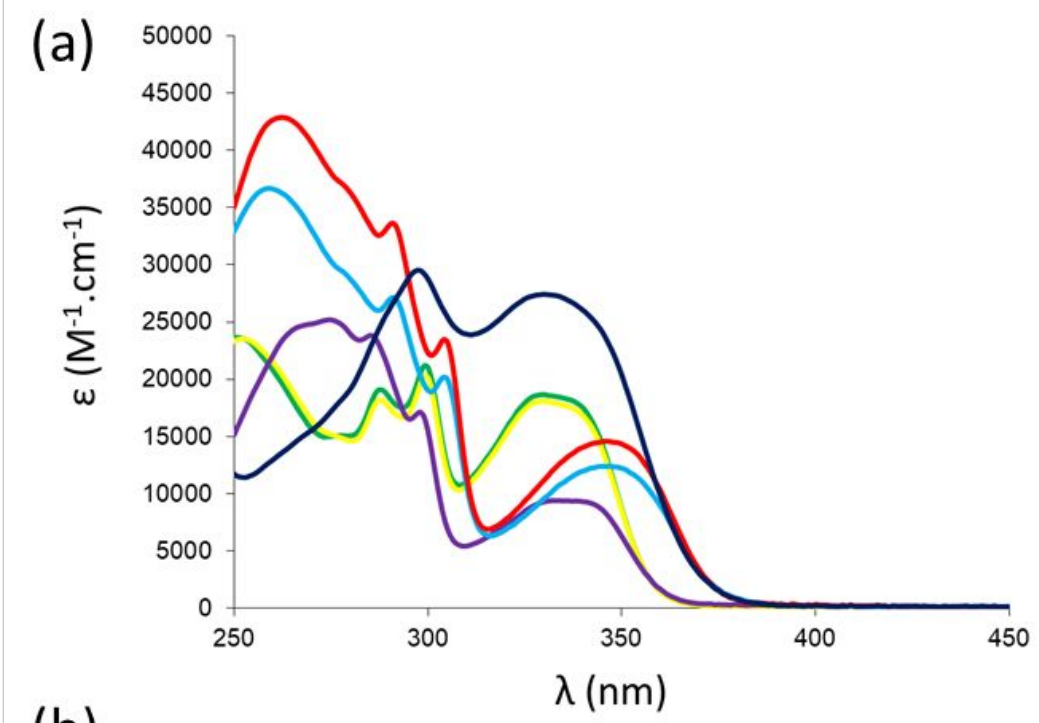

(b)

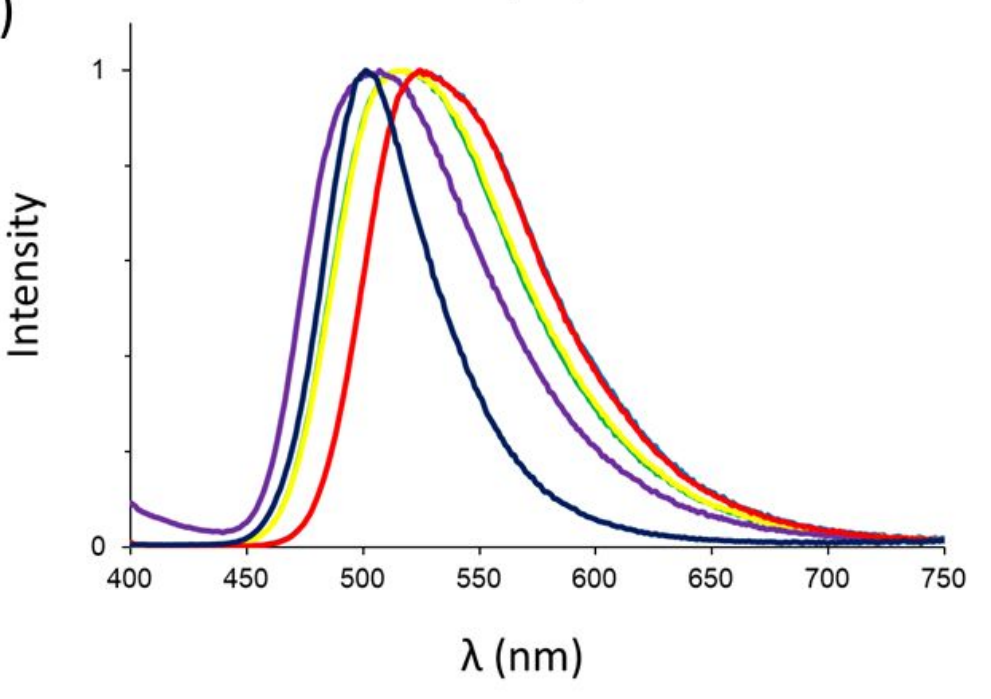

Figure S74. Emission spectra of HBO 1 (green), HBO 2 (yellow), HBO 3 (purple), HBO 5 (blue), HBO 6 (red), HBO 8 (pink) in their protonated state in ethanol at $25^{\circ} \mathrm{C}$. 


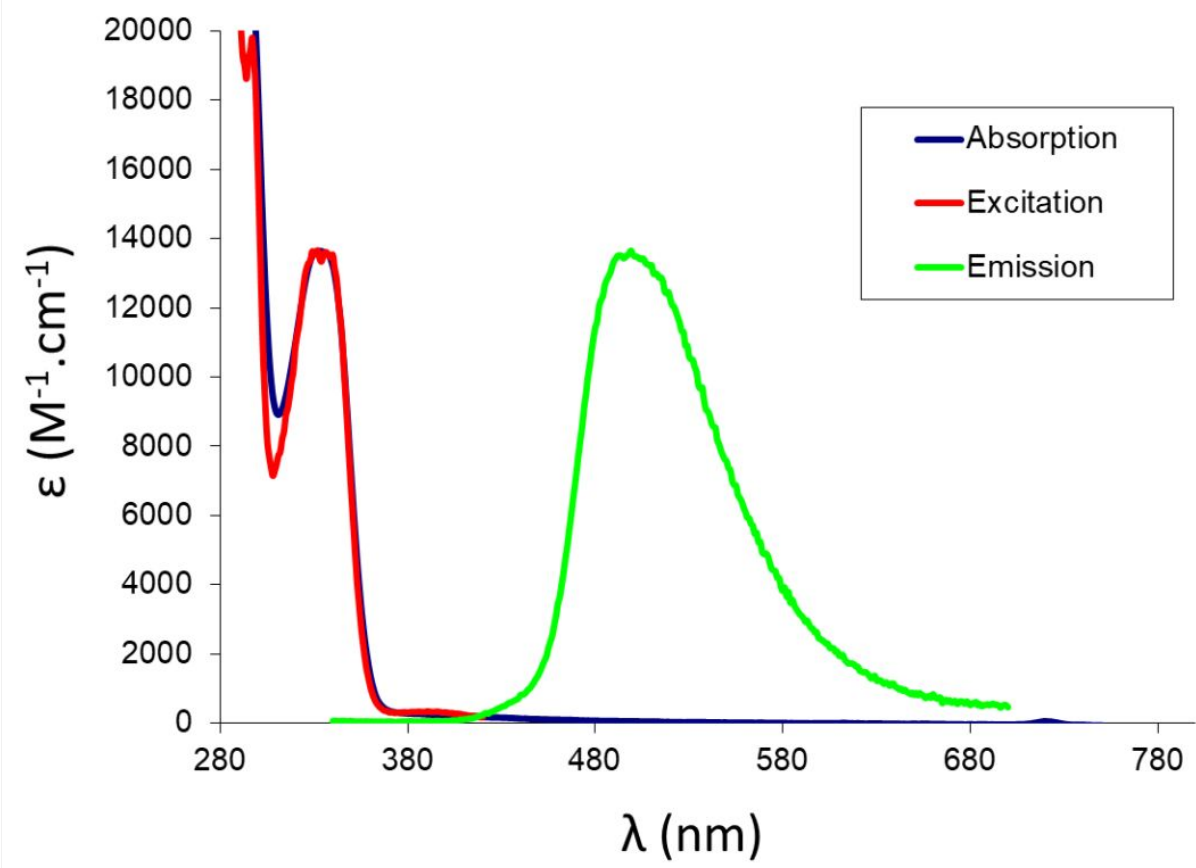

Figure S75. Absorption (blue), emission (green) and excitation (red) of $\mathrm{HBO}$ dye 8 at $25^{\circ} \mathrm{C}$, in dichloromethane, in the presence of triethylamine.

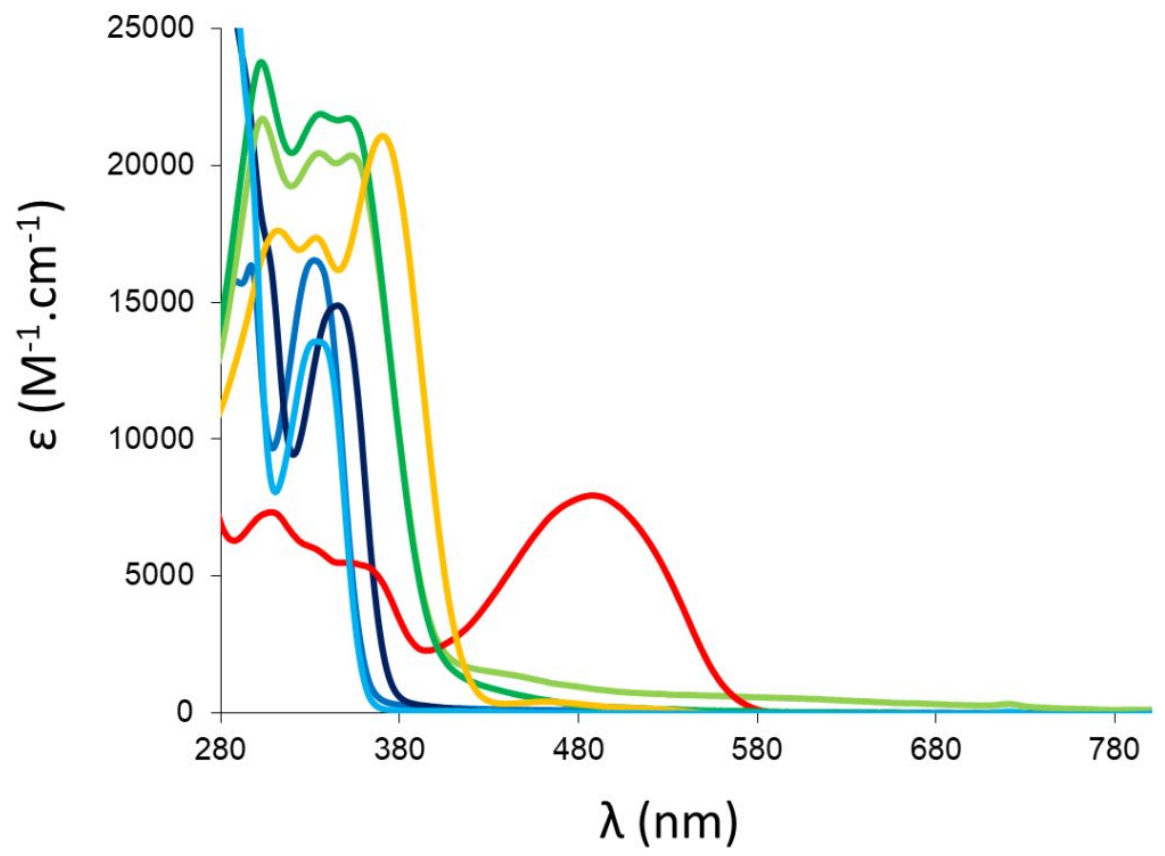

Figure S76. Absorption of HBO dye 8/10 (light blue), HBO 11 (blue), HBO 12 (navy blue), HBO 13 (green), HBO 14 (light green), HBO 16 (orange) and HBO 17 (red) in dichloromethane at $25^{\circ} \mathrm{C}$. 
Table S2. Photophysical data of aerated solutions of dyes 8, 10-13 and 15-16, in dichloromethane with $0.1 \%$ of $\mathrm{NEt}_{3}$ at $25^{\circ} \mathrm{C}$.

\begin{tabular}{|c|c|c|c|c|c|c|c|c|}
\hline Dye & $\begin{array}{l}\lambda_{\mathrm{abs}} \\
(\mathrm{nm})\end{array}$ & $\begin{array}{c}\varepsilon \\
\left(M^{-1} . \mathrm{cm}^{-1}\right)\end{array}$ & $\begin{array}{c}\lambda_{\mathrm{em}} \\
(\mathrm{nm})\end{array}$ & $\Phi_{\mathrm{f}}^{[\mathrm{a}]}$ & $\Delta_{\mathrm{ss}}^{[\mathrm{b}]}$ & $\begin{array}{c}\mathrm{T} \\
\text { (ns) }\end{array}$ & $\mathrm{K}_{\mathrm{r}}[\mathrm{c}]$ & $\mathrm{K}_{\mathrm{nr}}{ }^{[c]}$ \\
\hline 8 & 331 & 14000 & 499 & 0.10 & 10000 & 3.4 & 0.3 & 2.7 \\
\hline 10 & 335 & 15900 & 518 & 0.37 & 10500 & 1.2 & 3.1 & 5.3 \\
\hline 11 & 348 & 15500 & 519 & 0.50 & 9500 & 3.7 & 1.4 & 1.4 \\
\hline 12 & $349 / 502$ & 32500 & 500 & 0.01 & 8700 & 2.9 & 0.1 & 11.0 \\
\hline 13 & $351 / 501$ & 24700 & 503 & 0.04 & 8500 & 0.8 & 0.5 & 12.0 \\
\hline 15 & 522 & 43700 & - & - & - & - & - & - \\
\hline 16 & 508 & 18400 & - & - & - & - & - & - \\
\hline
\end{tabular}

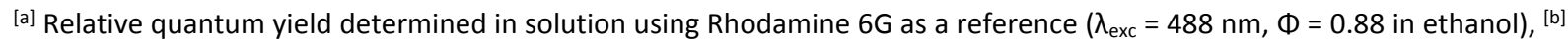
Stokes shift, ${ }^{[c]} k_{r}\left(10^{8} s^{-1}\right)$ and $k_{n r}\left(10^{8} s^{-1}\right)$ were calculated using: $k_{r}=\Phi_{F} / \sigma, k_{n r}=\left(1-\Phi_{F}\right) / \sigma$ where $\sigma$ is the lifetime.

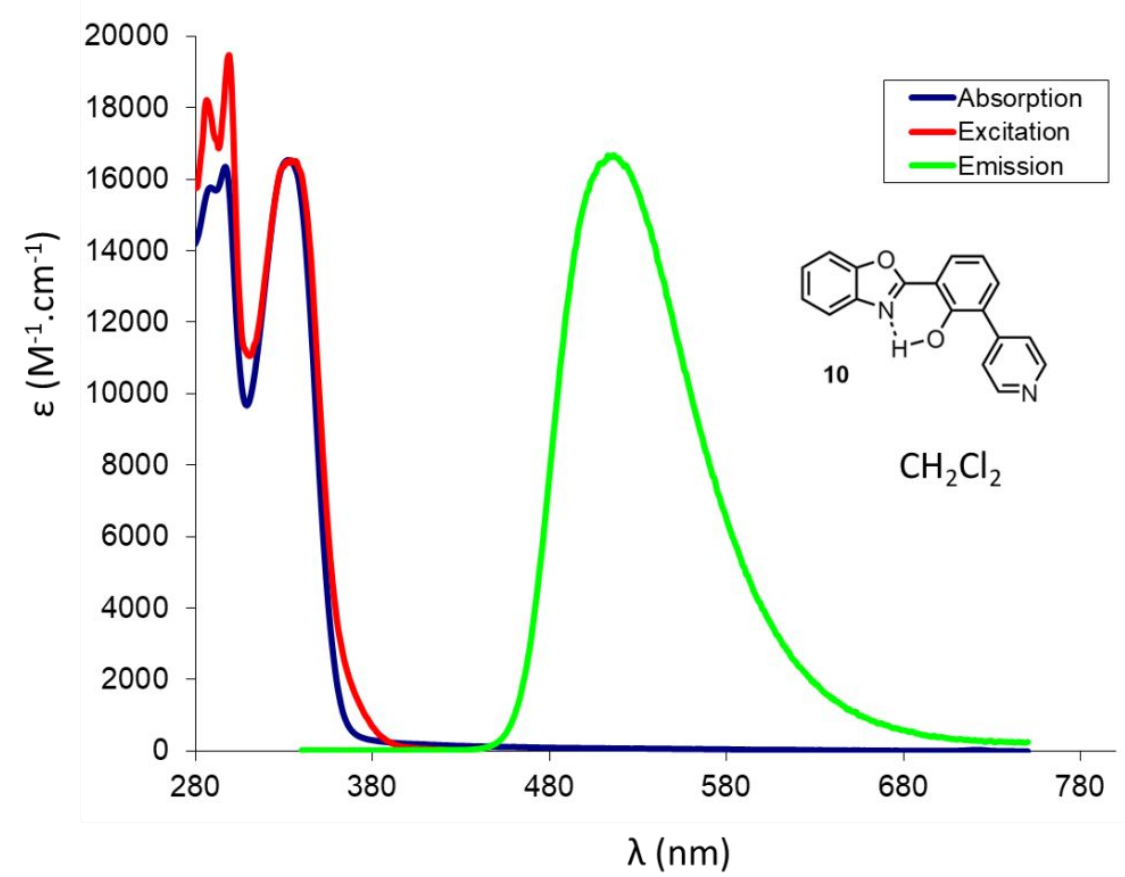

Figure S77. Absorption (blue), emission (green) and excitation (red) of $\mathrm{HBO}$ dye $\mathbf{1 0}$ at $25^{\circ} \mathrm{C}$, in dichloromethane. 


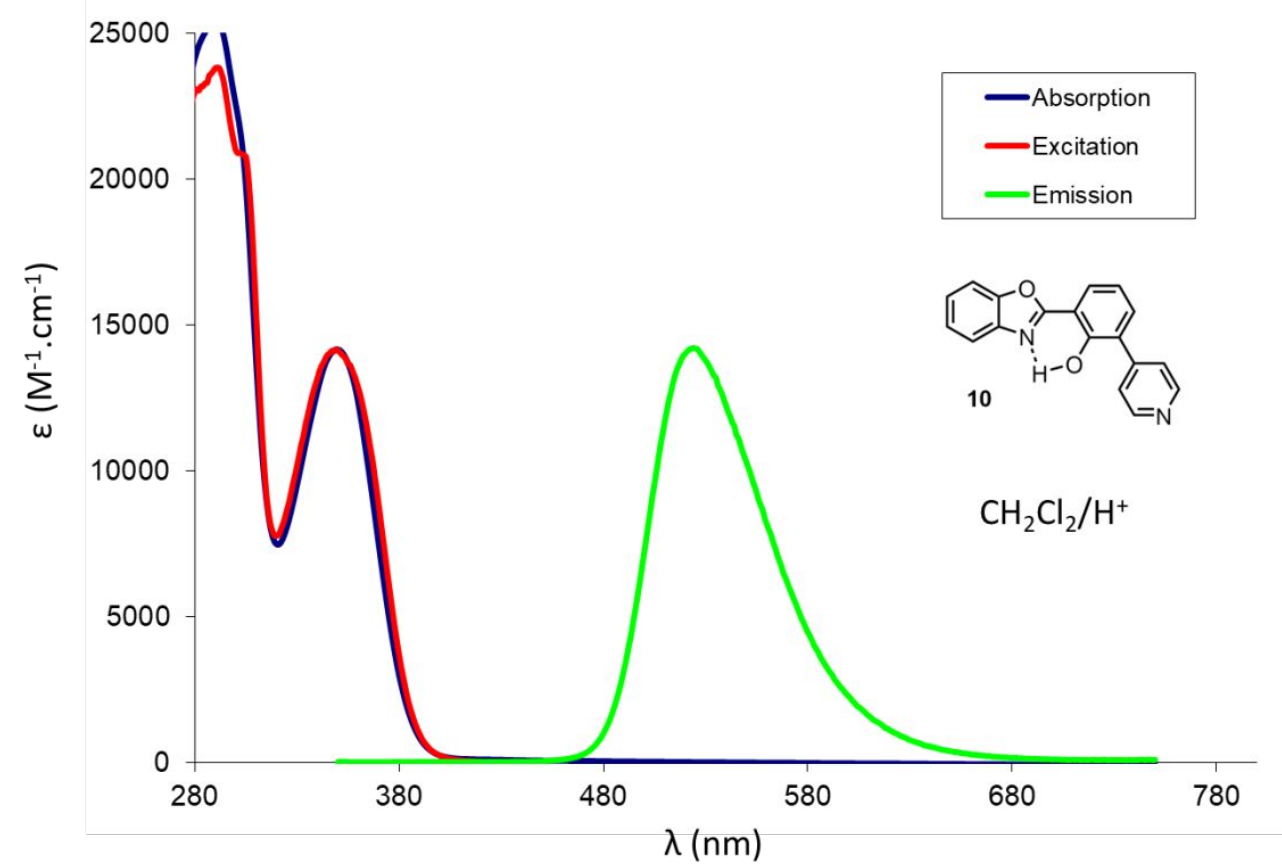

Figure S78. Absorption (blue), emission (green) and excitation (red) of $\mathrm{HBO}$ dye $\mathbf{1 0}$ at $25^{\circ} \mathrm{C}$, in protonated dichloromethane.

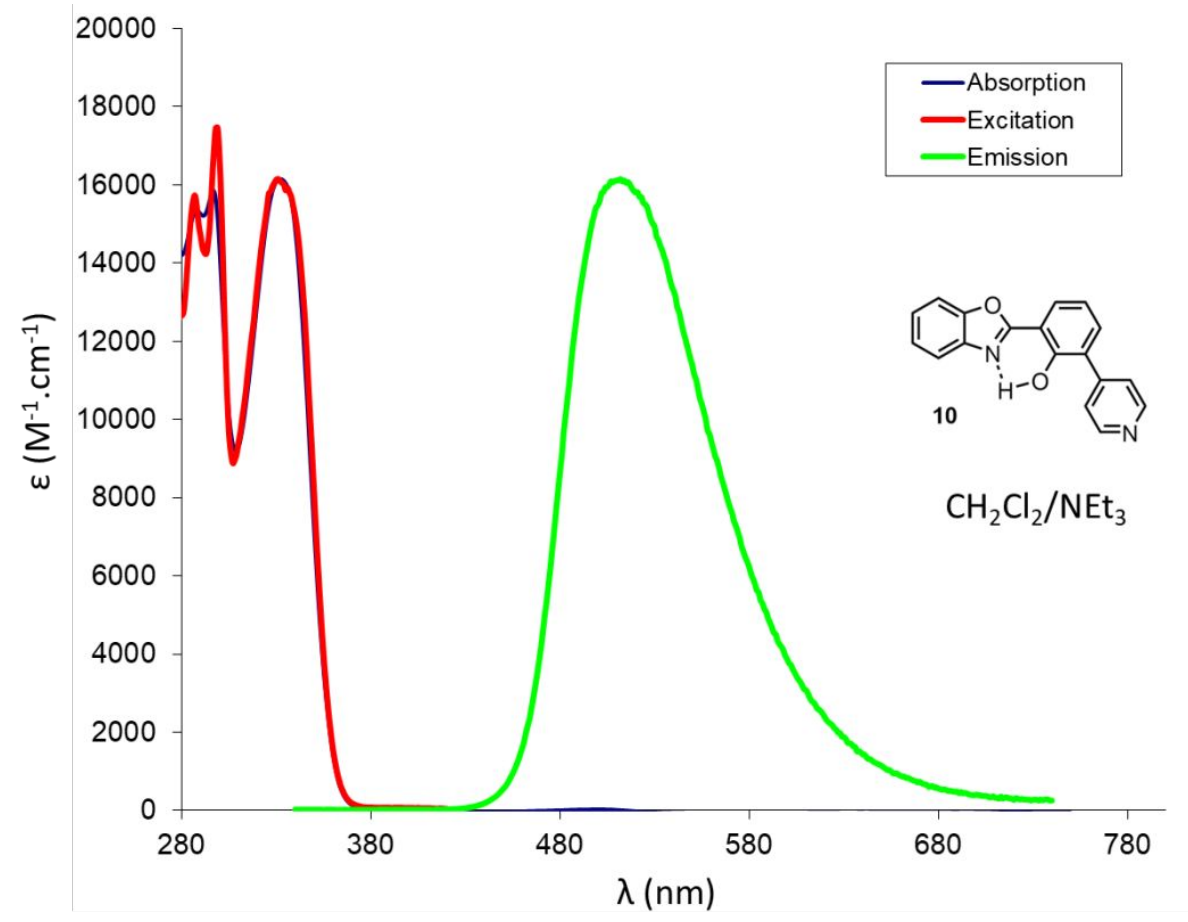

Figure S79. Absorption (blue), emission (green) and excitation (red) of $\mathrm{HBO}$ dye $\mathbf{1 0}$ at $25^{\circ} \mathrm{C}$, in dichloromethane, in the presence of triethylamine. 


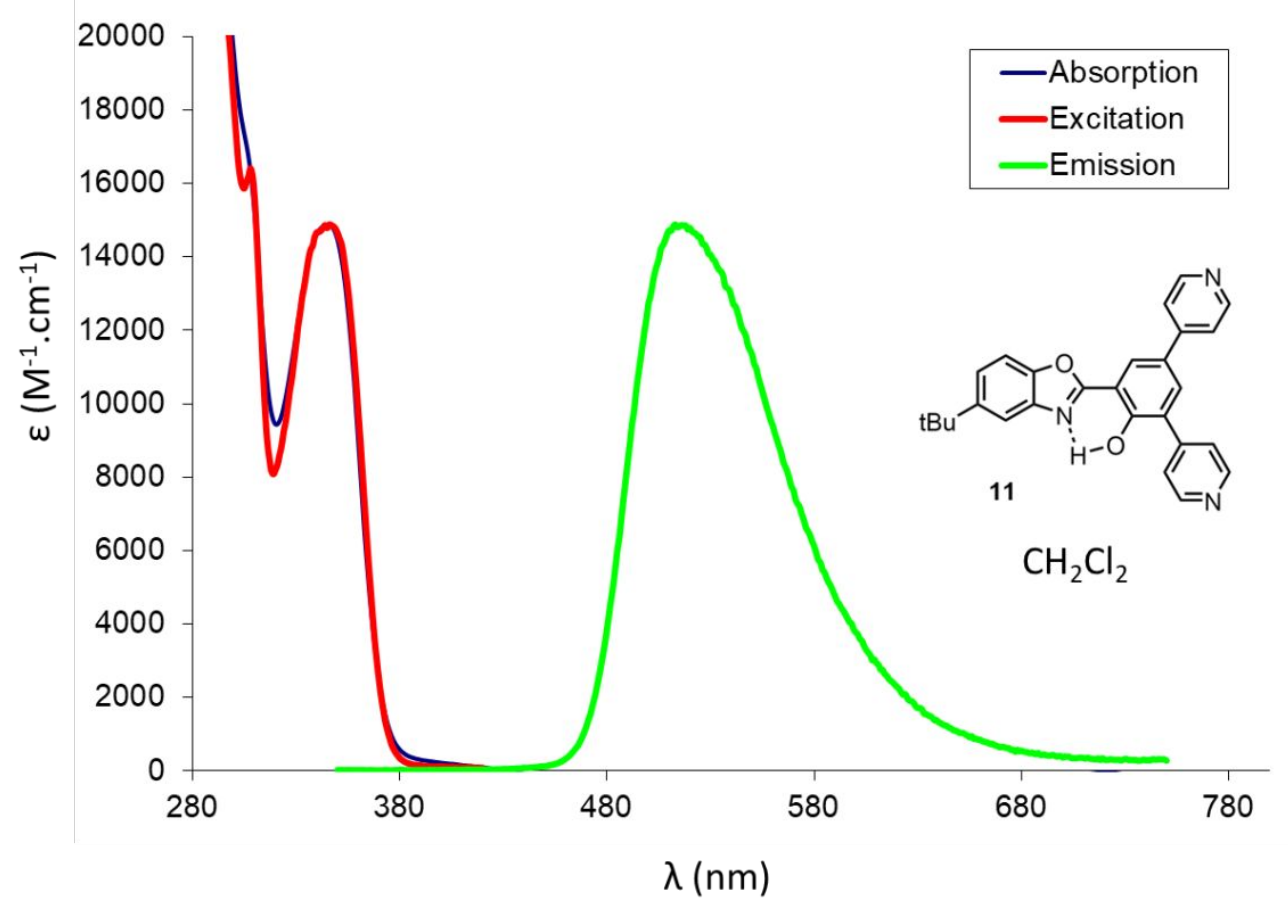

Figure S80. Absorption (blue), emission (green) and excitation (red) of $\mathrm{HBO}$ dye $\mathbf{1 1}$ at $25^{\circ} \mathrm{C}$, in dichloromethane.

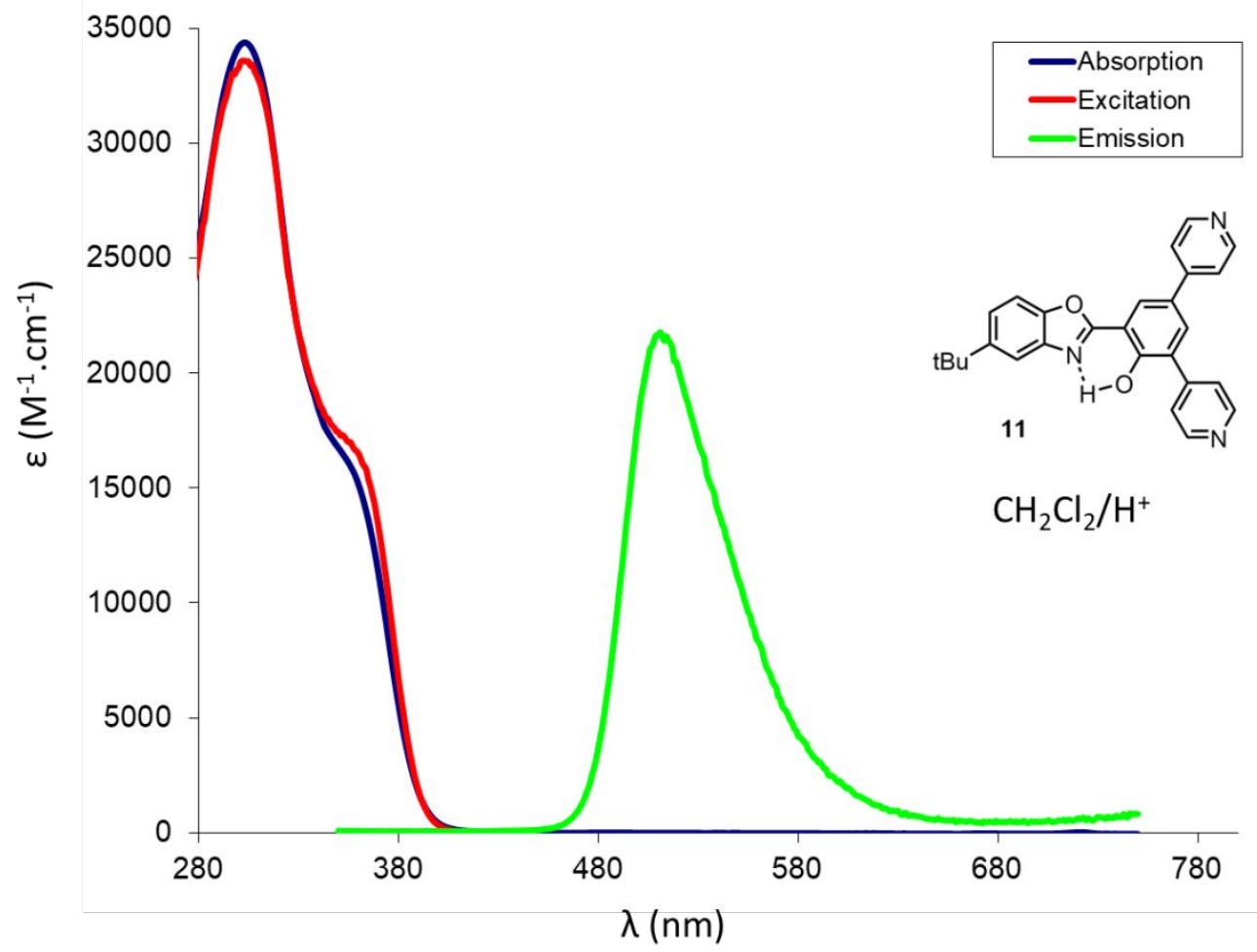

Figure S81. Absorption (blue), emission (green) and excitation (red) of $\mathrm{HBO}$ dye $\mathbf{1 1}$ at $25^{\circ} \mathrm{C}$, in protonated dichloromethane. 


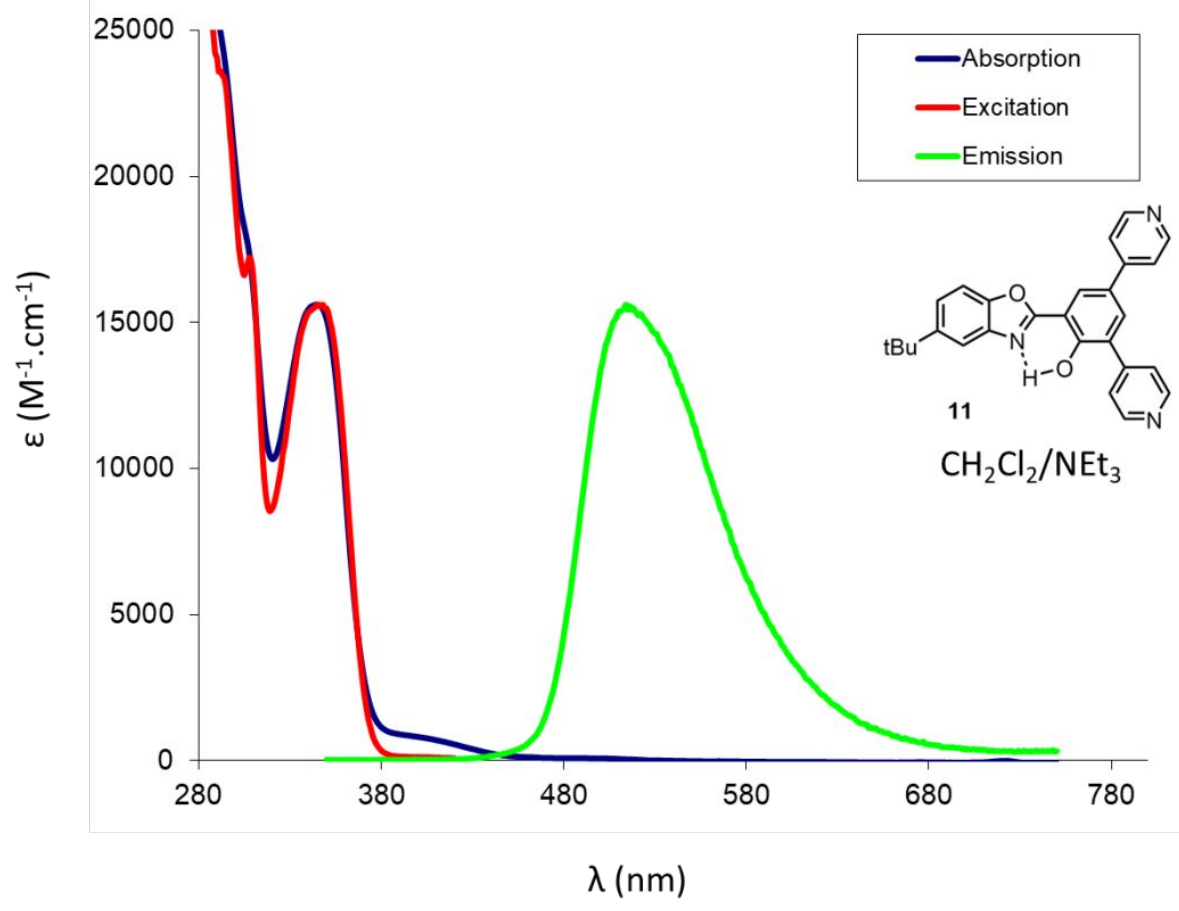

Figure S82. Absorption (blue), emission (green) and excitation (red) of $\mathrm{HBO}$ dye $\mathbf{1 1}$ at $25^{\circ} \mathrm{C}$, in dichloromethane, in the presence of triethylamine.

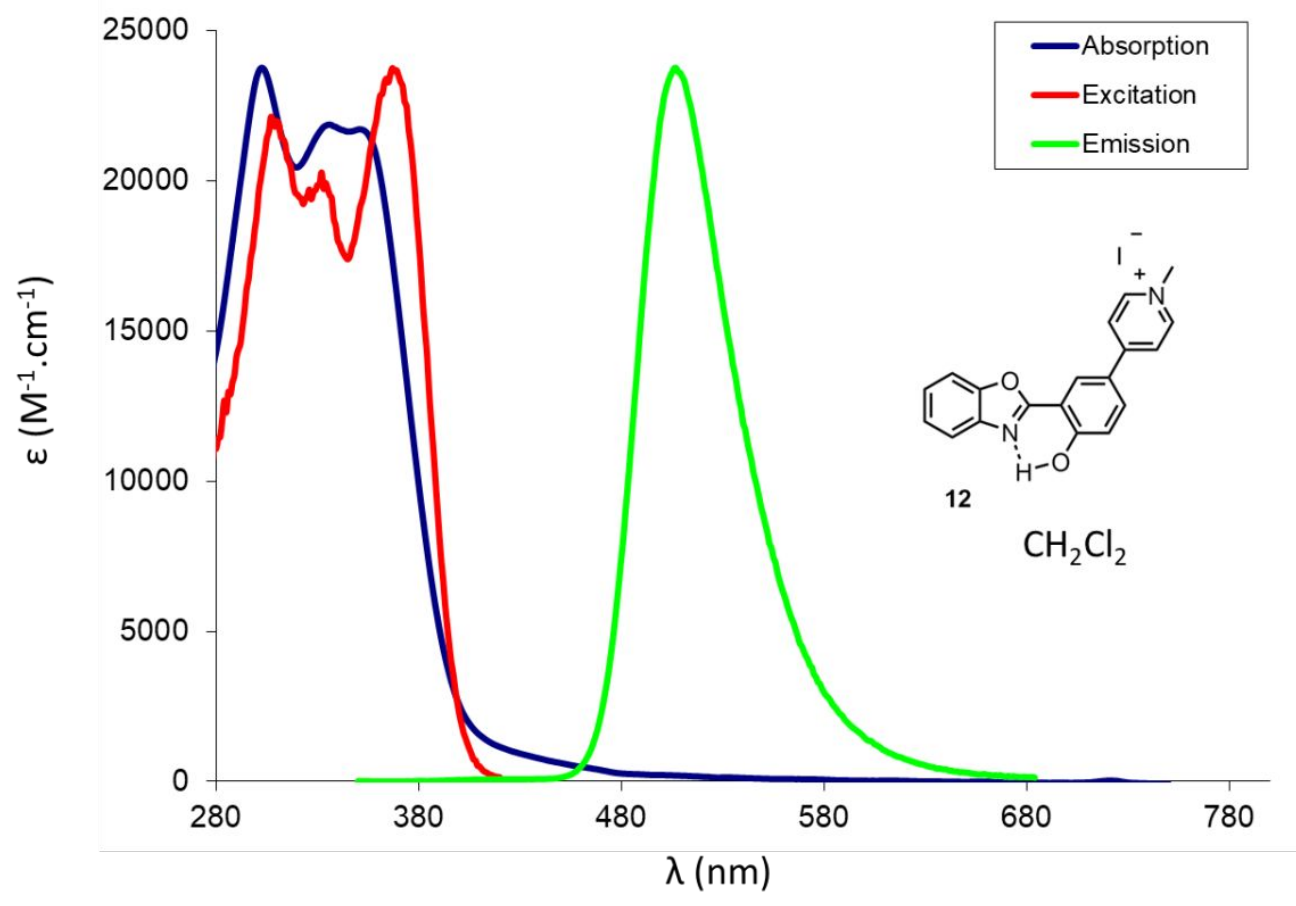

Figure S83. Absorption (blue), emission (green) and excitation (red) of $\mathrm{HBO}$ dye 12 at $25^{\circ} \mathrm{C}$, in dichloromethane. 


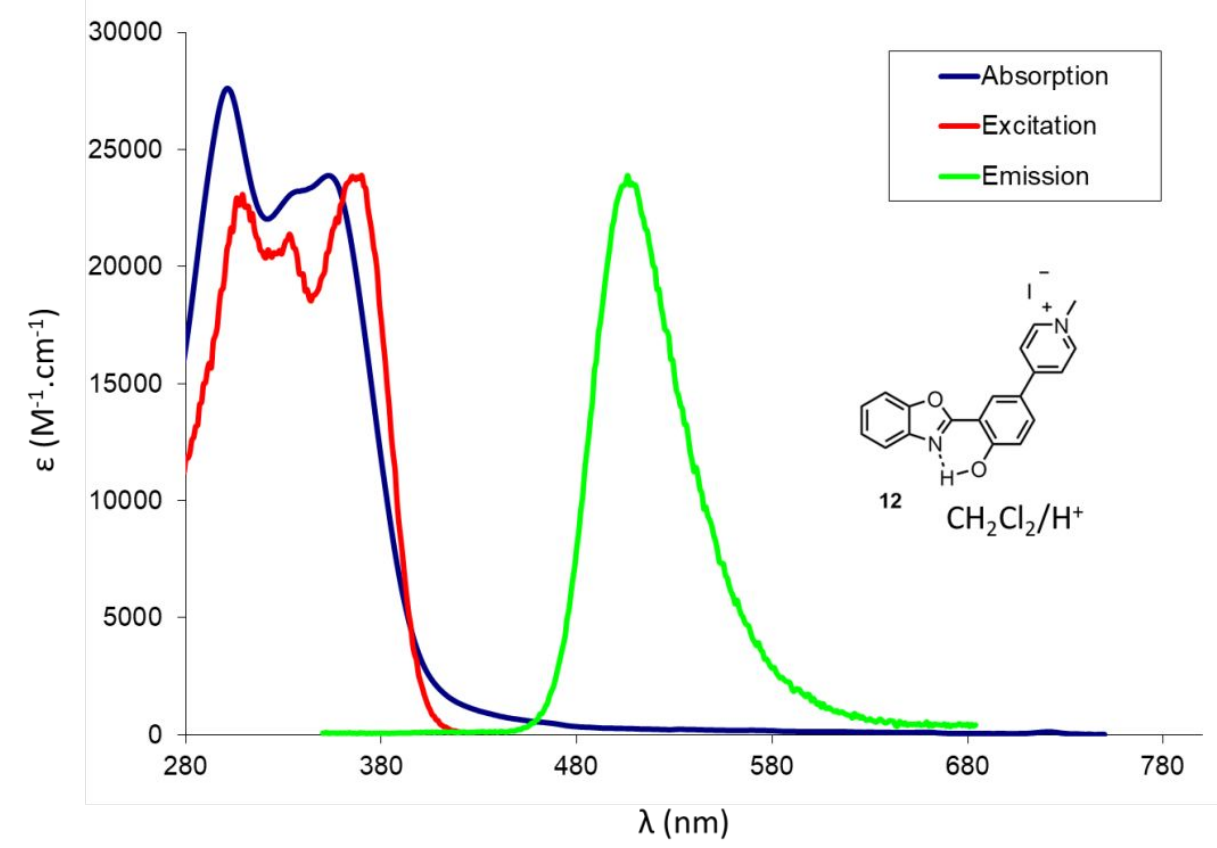

Figure S84. Absorption (blue), emission (green) and excitation (red) of $\mathrm{HBO}$ dye $\mathbf{1 2}$ at $25^{\circ} \mathrm{C}$, in protonated dichloromethane.

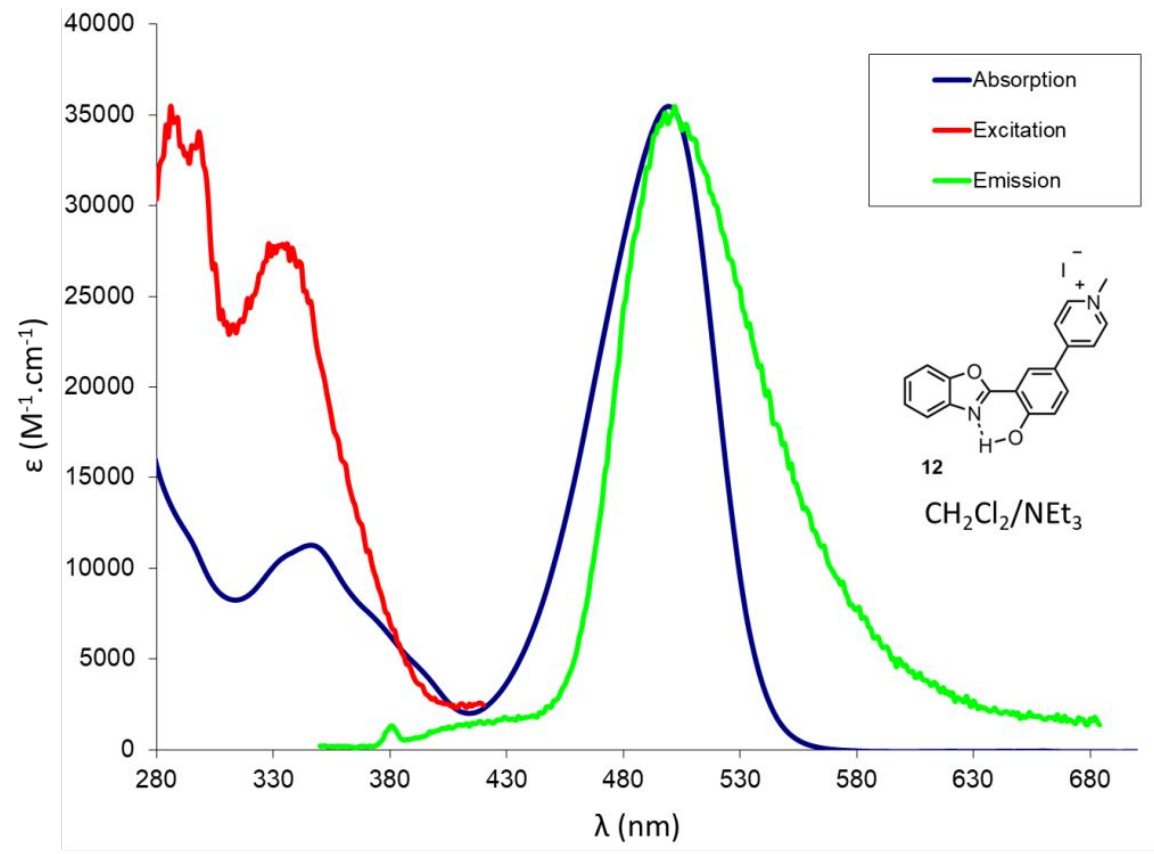

Figure S85. Absorption (blue), emission (green) and excitation (red) of $\mathrm{HBO}$ dye $\mathbf{1 2}$ at $25^{\circ} \mathrm{C}$, in dichloromethane, in the presence of triethylamine. 


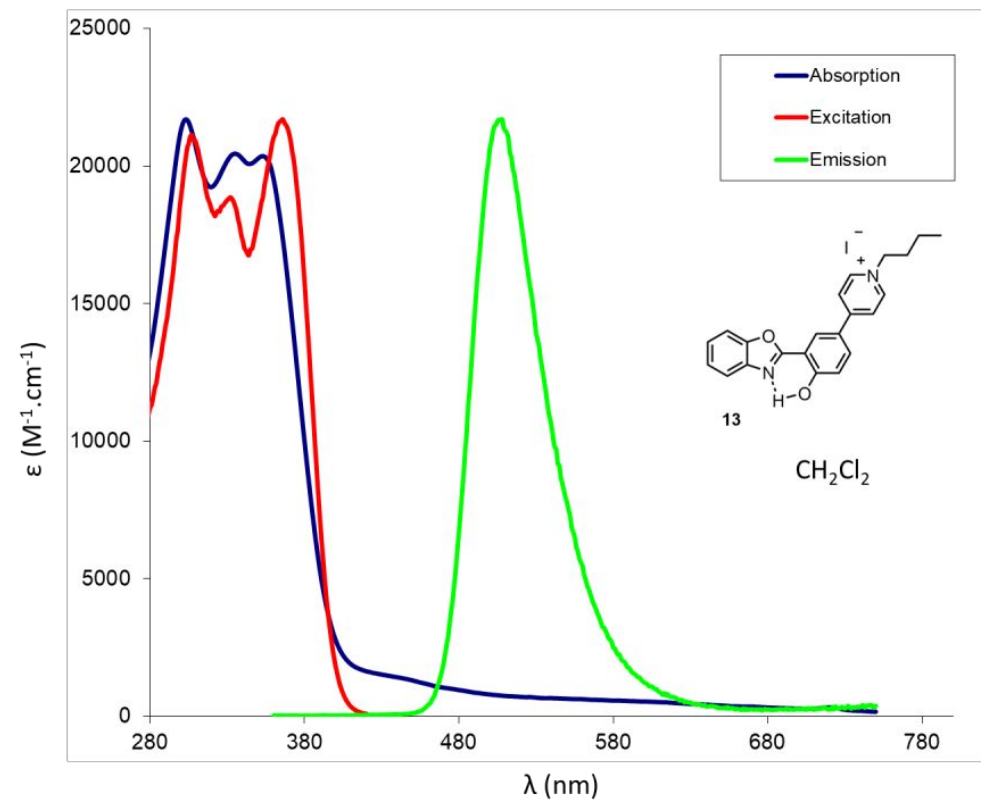

Figure S86. Absorption (blue), emission (green) and excitation (red) of $\mathrm{HBO}$ dye $\mathbf{1 3}$ at $25^{\circ} \mathrm{C}$, in dichloromethane.

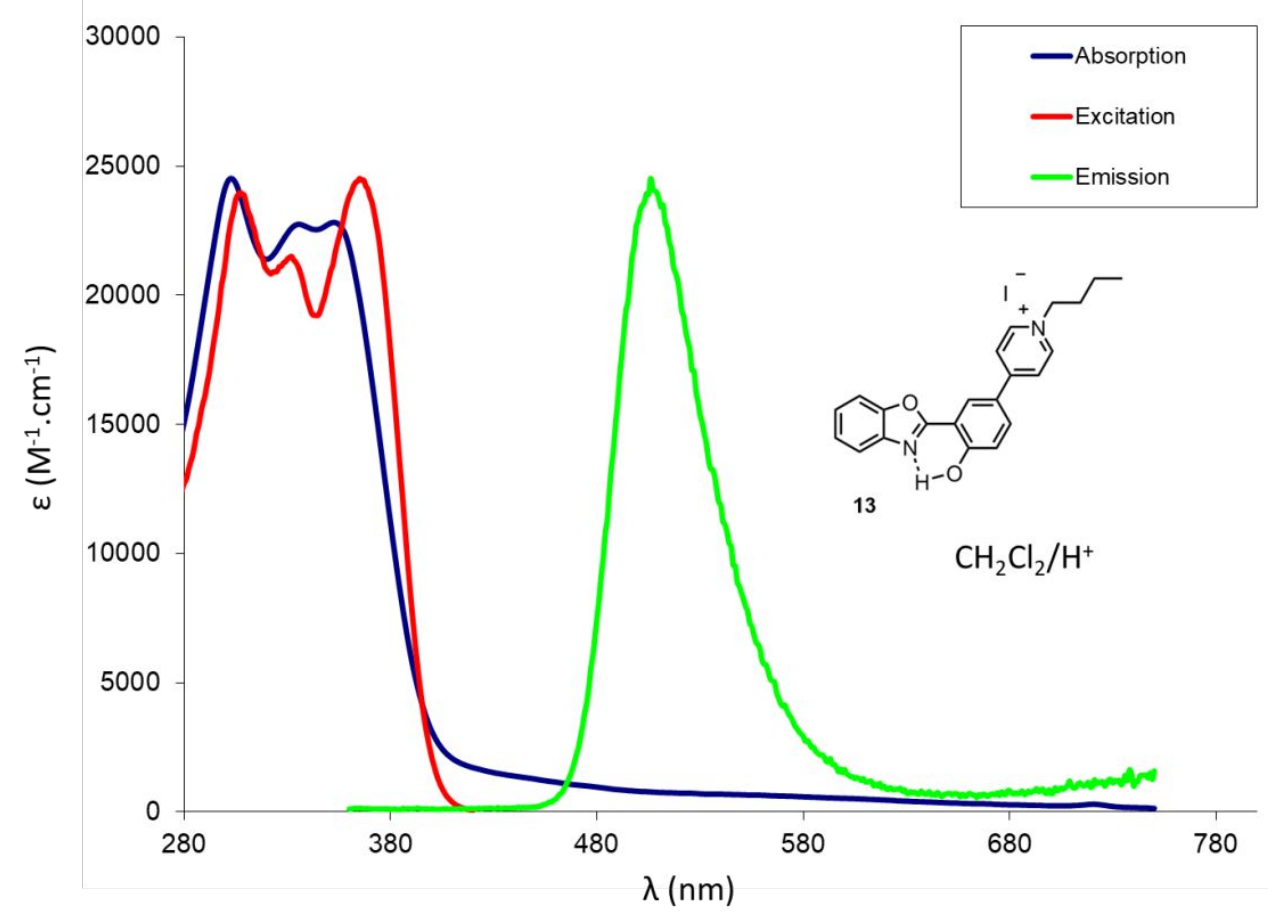

Figure S87. Absorption (blue), emission (green) and excitation (red) of $\mathrm{HBO}$ dye $\mathbf{1 3}$ at $25^{\circ} \mathrm{C}$, in protonated dichloromethane. 


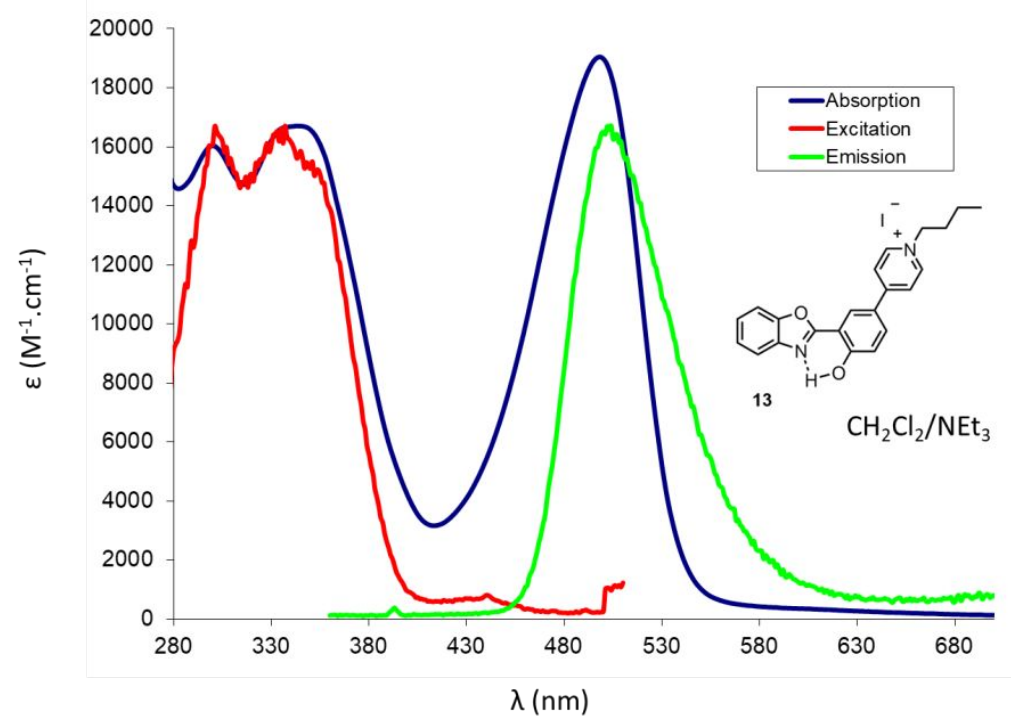

Figure S88. Absorption (blue), emission (green) and excitation (red) of $\mathrm{HBO}$ dye $\mathbf{1 3}$ at $25^{\circ} \mathrm{C}$, in dichloromethane, in the presence of triethylamine.

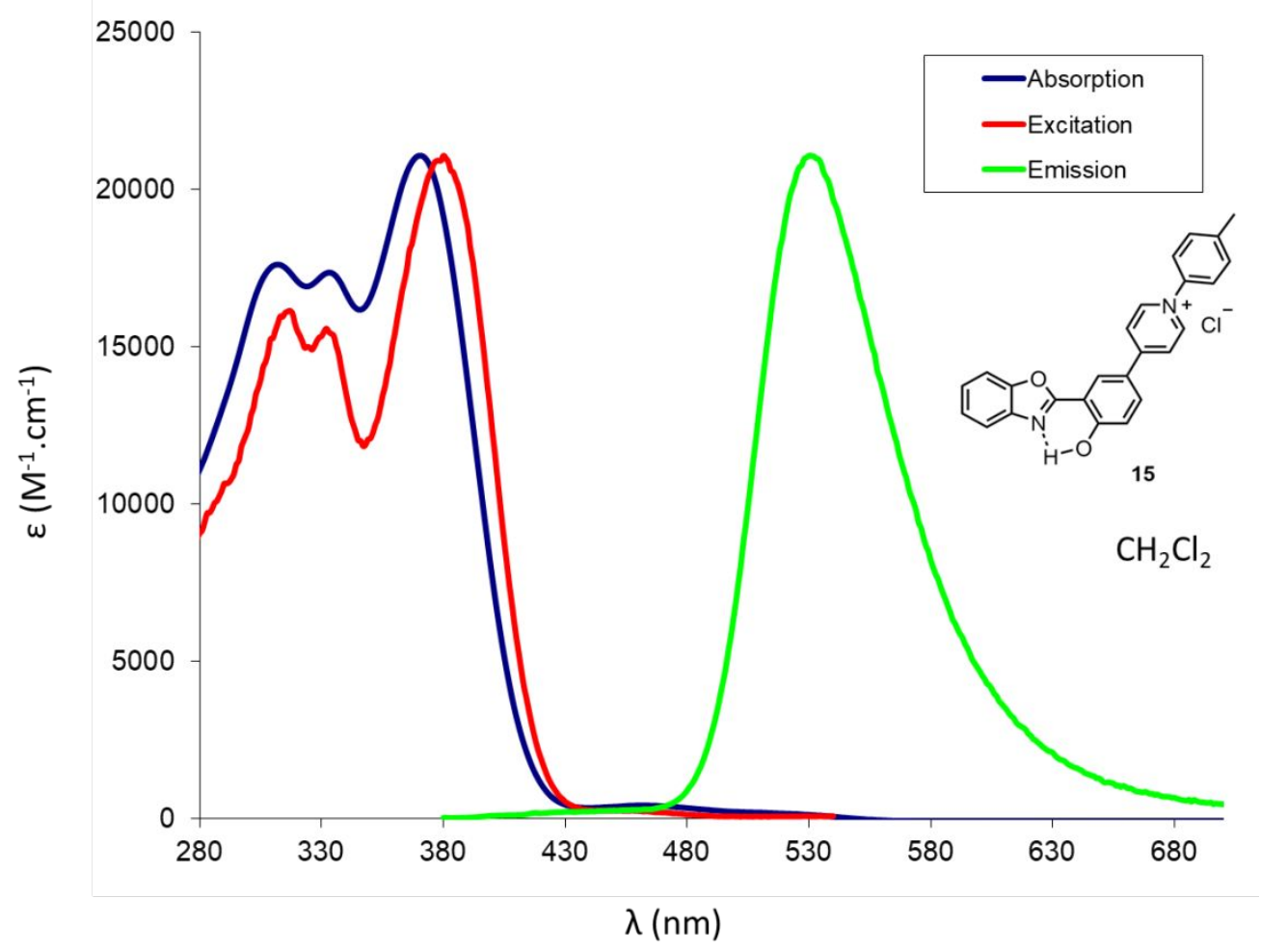

Figure S89. Absorption (blue), emission (green) and excitation (red) of $\mathrm{HBO}$ dye $\mathbf{1 5}$ at $25^{\circ} \mathrm{C}$, in dichloromethane. 


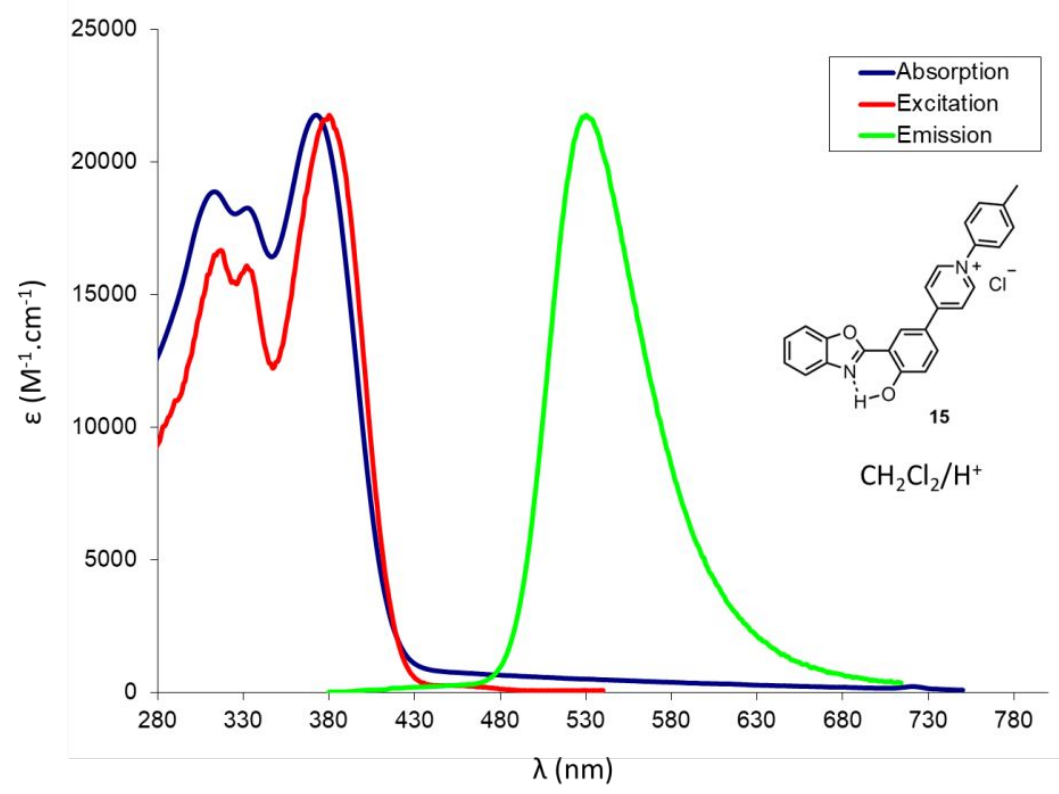

Figure S90. Absorption (blue), emission (green) and excitation (red) of $\mathrm{HBO}$ dye $\mathbf{1 5}$ at $25^{\circ} \mathrm{C}$, in protonated dichloromethane.

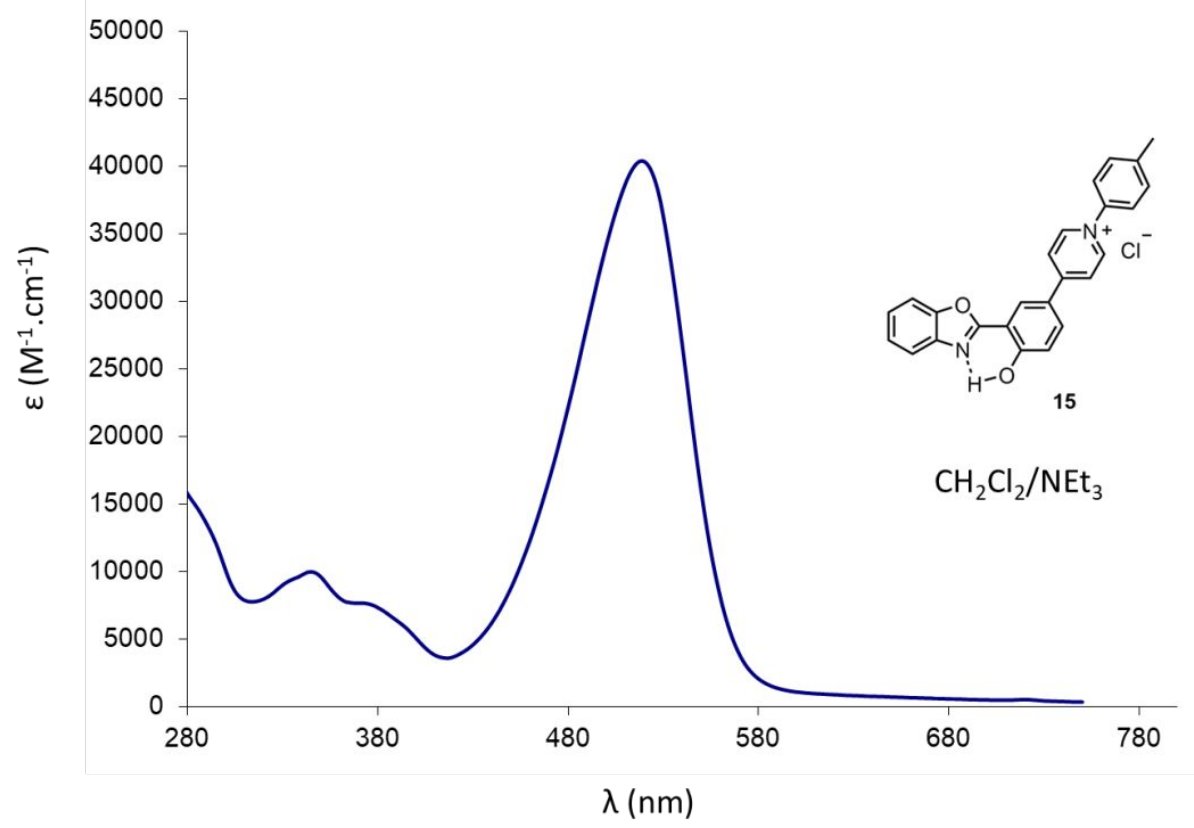

Figure S91. Absorption of $\mathrm{HBO}$ dye 15 at $25^{\circ} \mathrm{C}$, in dichloromethane, in the presence of triethylamine. 


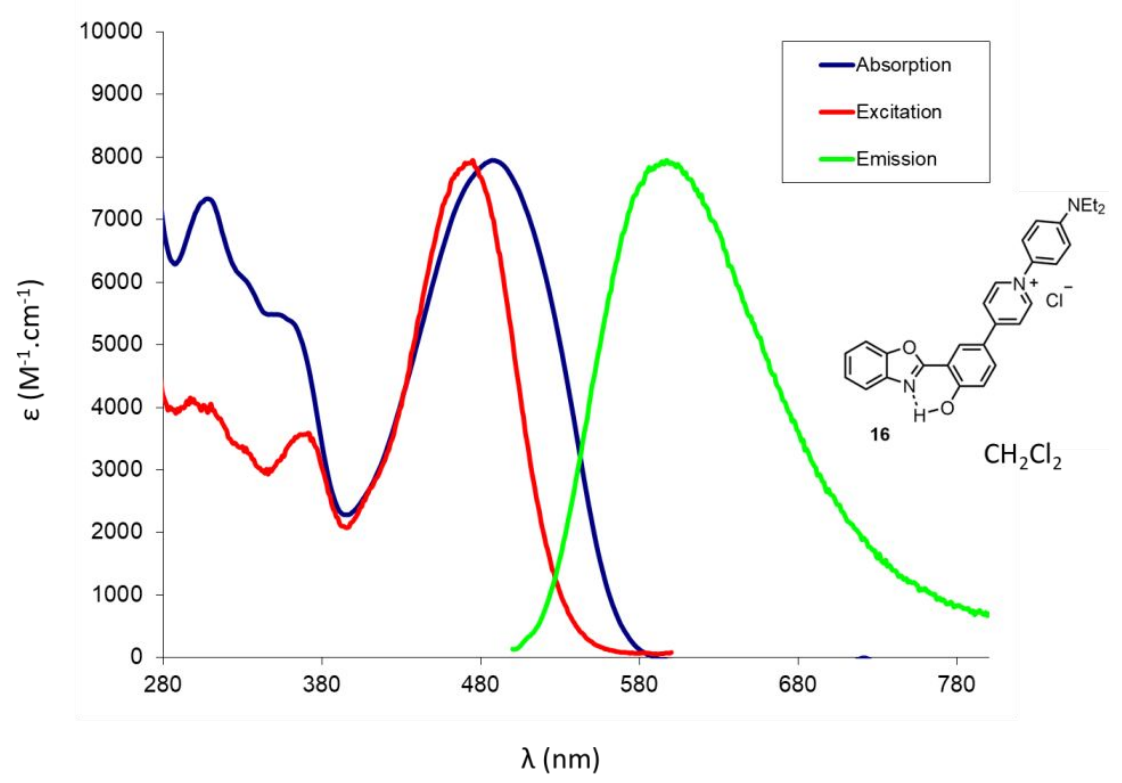

Figure S92. Absorption (blue), emission (green) and excitation (red) of $\mathrm{HBO}$ dye 16 at $25^{\circ} \mathrm{C}$, in dichloromethane.

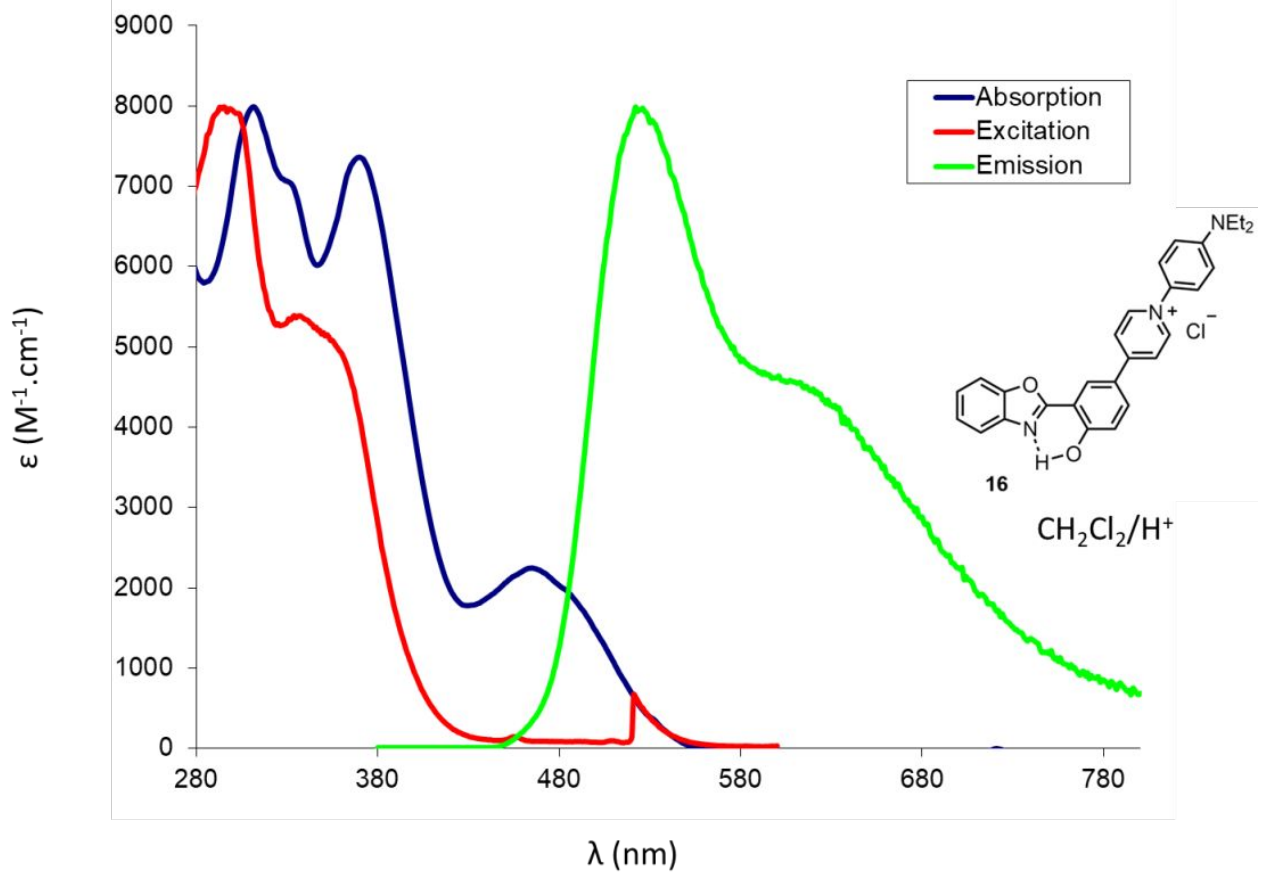

Figure S93. Absorption (blue), emission (green) and excitation (red) of $\mathrm{HBO}$ dye 16 at $25^{\circ} \mathrm{C}$, in protonated dichloromethane. 


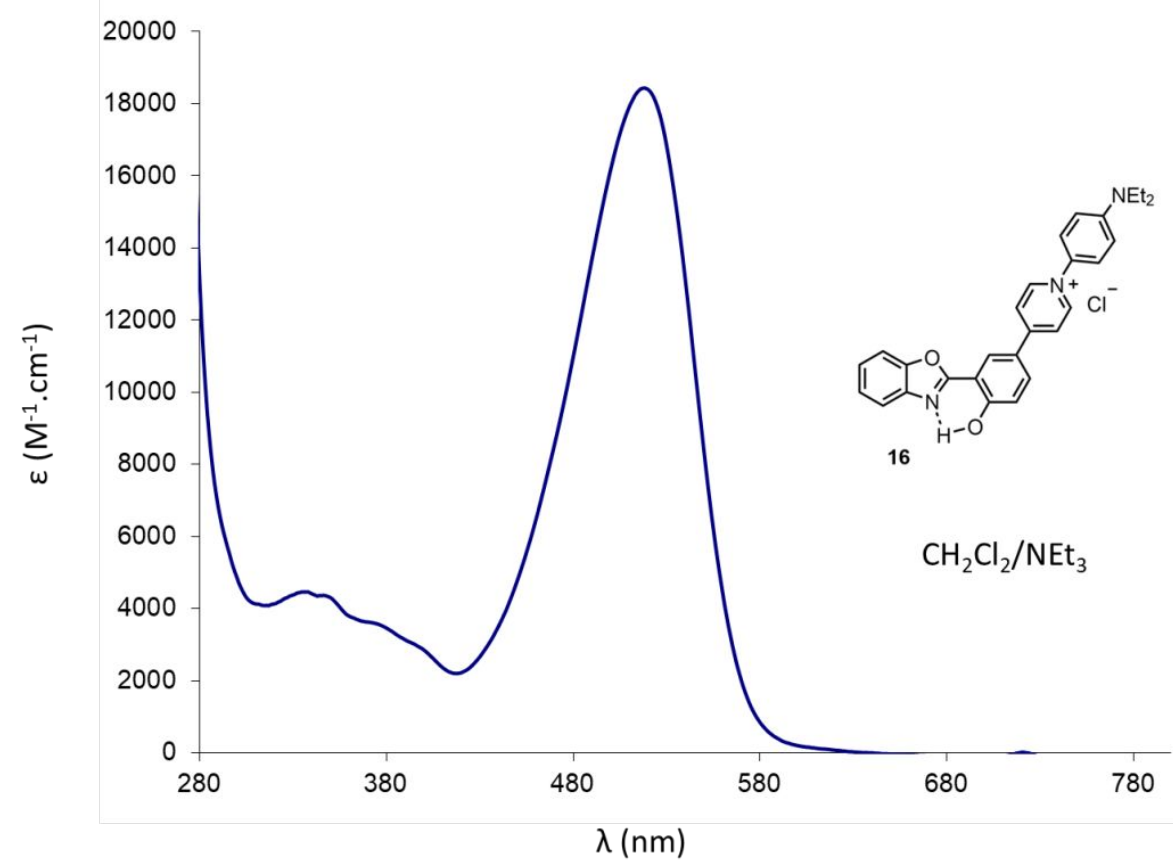

Figure S94. Absorption of $\mathrm{HBO}$ dye 16 at $25^{\circ} \mathrm{C}$, in dichloromethane, in the presence of triethylamine.

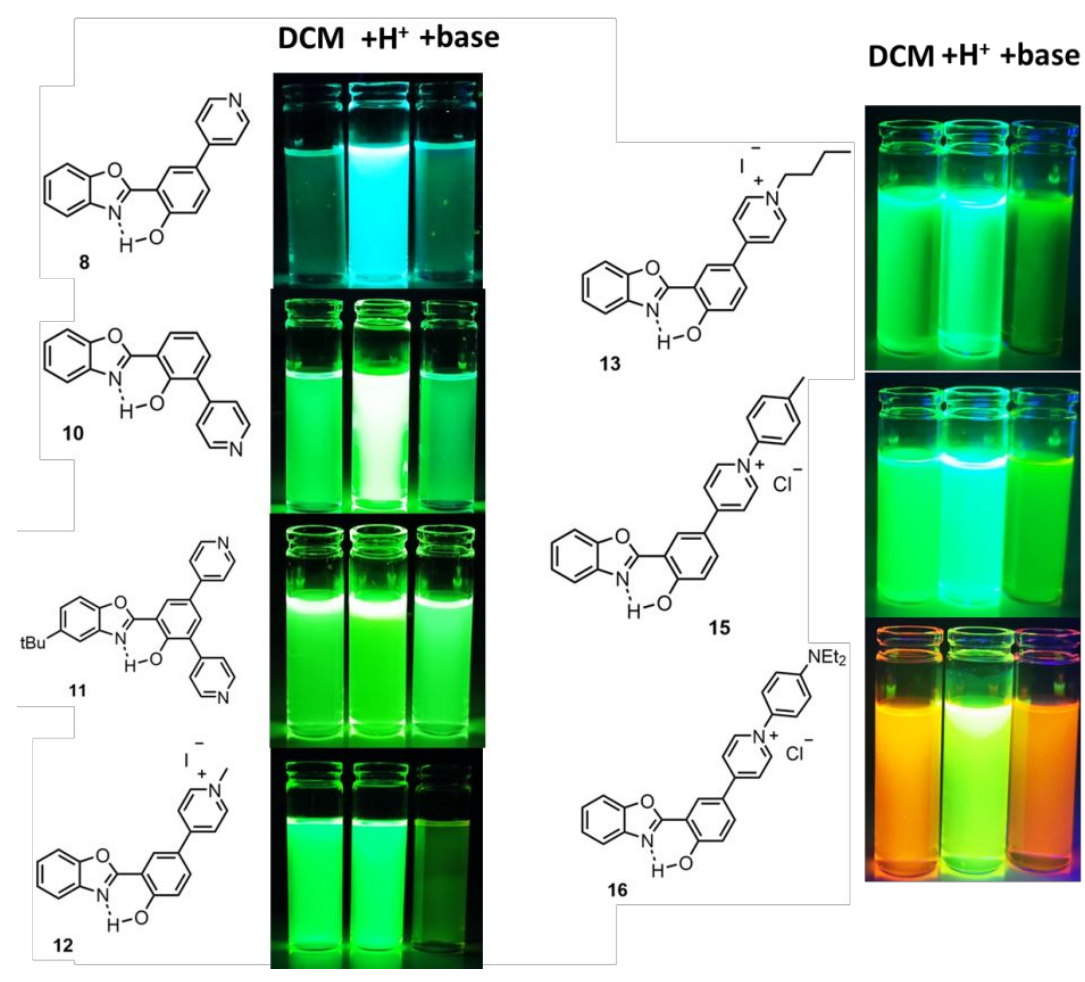

Figure S95. Pictures of dichloromethane solution of dyes 8, 11-13, 15 and 16 under irradiation ( $\lambda_{\mathrm{exc}}=$ $365 \mathrm{~nm}$ ) (left column), after addition of acid (central column) and base (right column). 


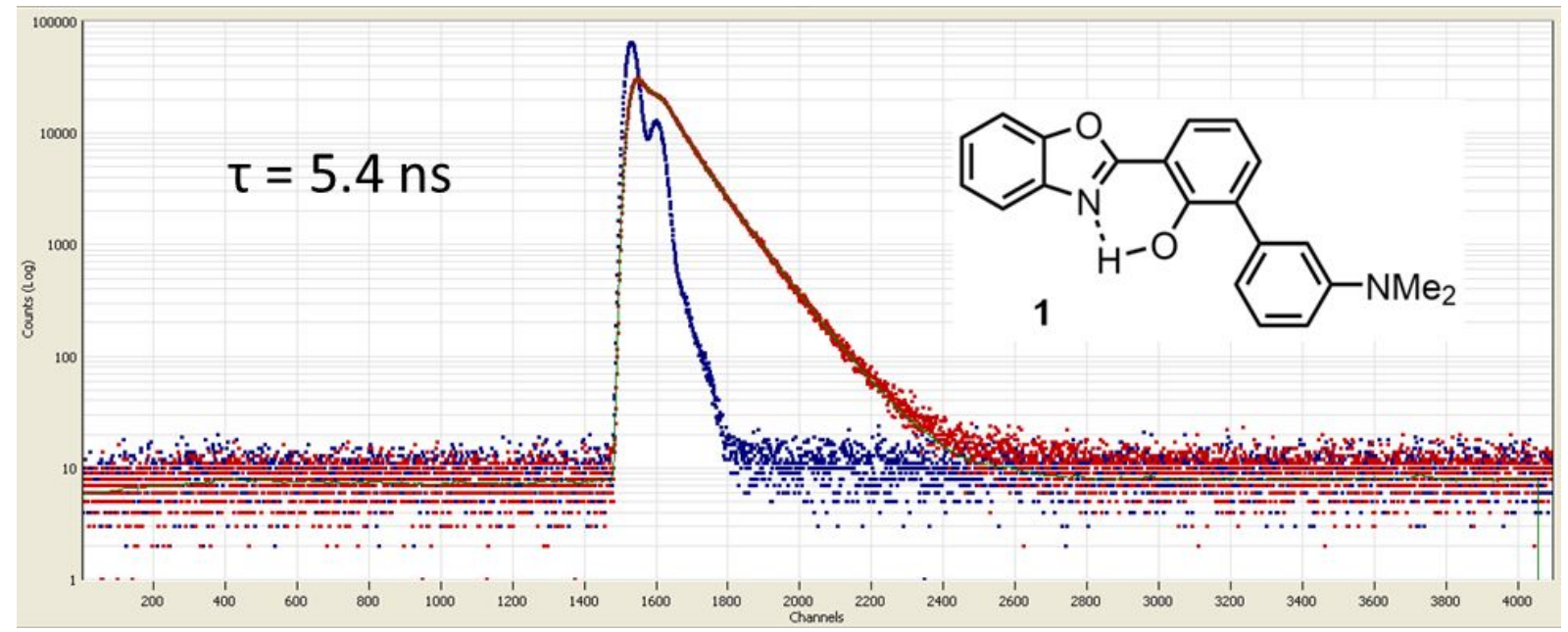

Figure S96. Time-resolved Fluorescence decays curved for dye 1 in DCM.

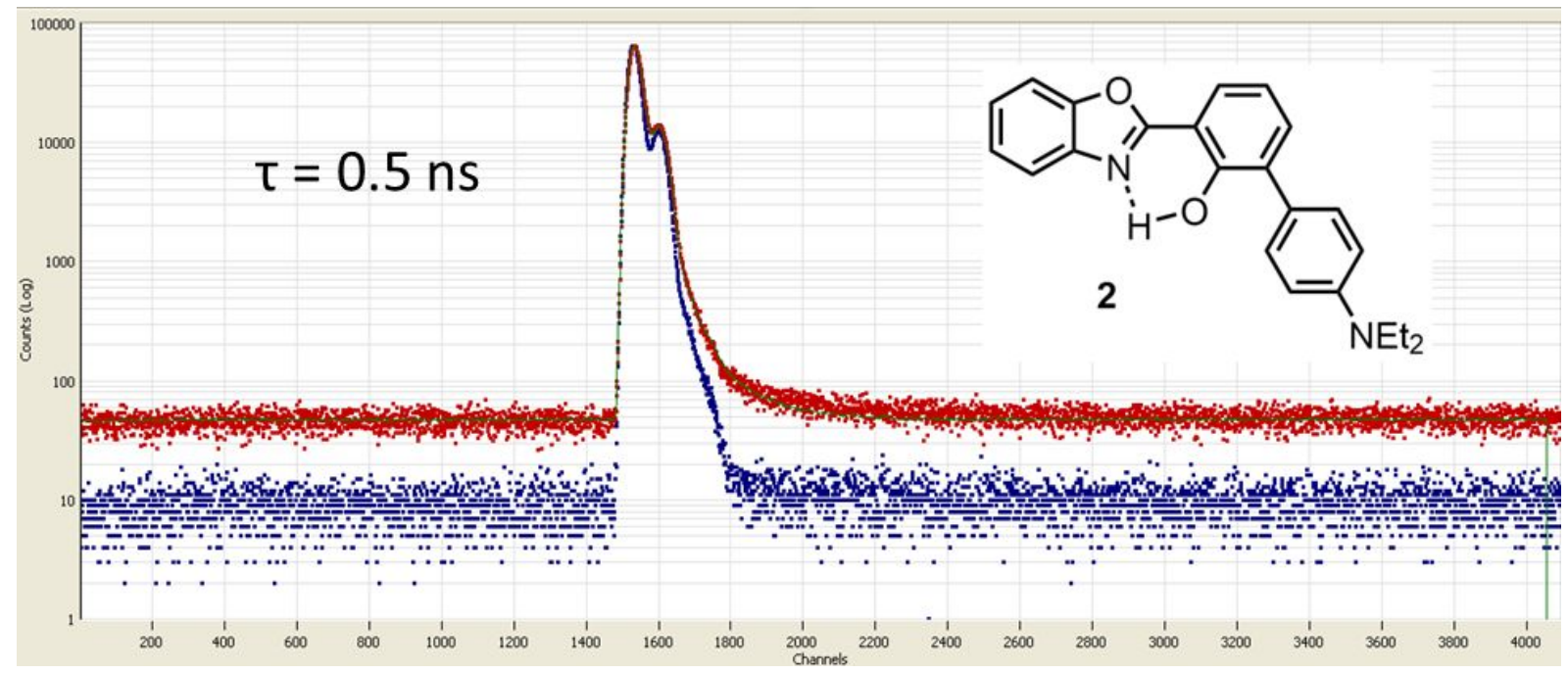

Figure S97. Time-resolved Fluorescence decays curved for dye $\mathbf{2}$ in DCM.

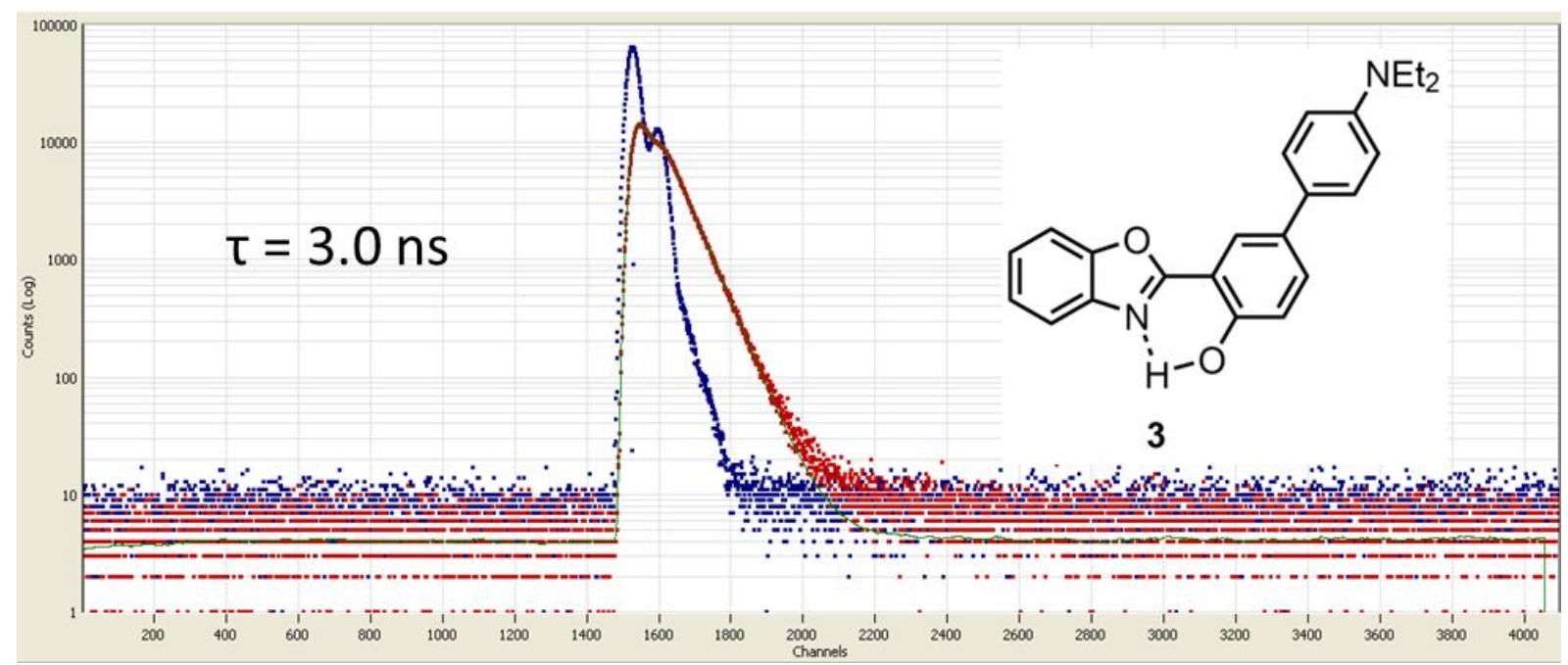

Figure S98. Time-resolved Fluorescence decays curved for dye $\mathbf{3}$ in DCM. 


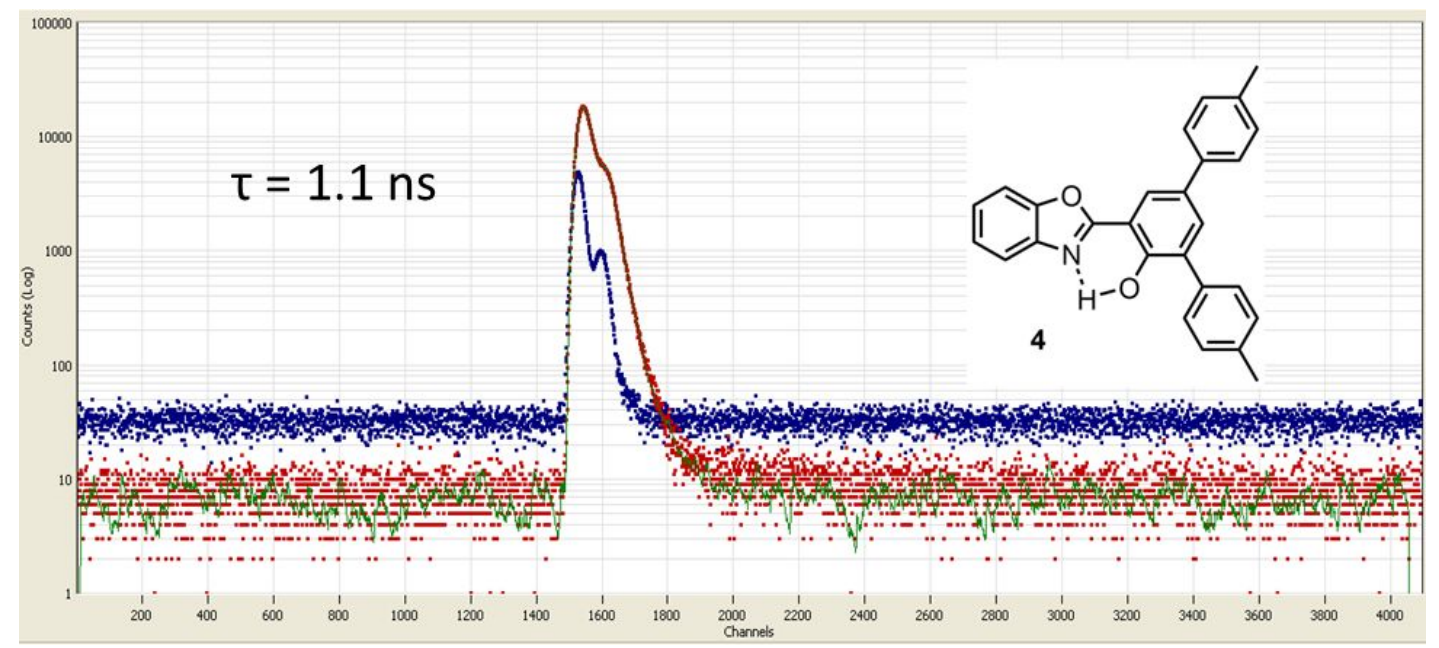

Figure S99. Time-resolved Fluorescence decays curved for dye $\mathbf{4}$ in DCM

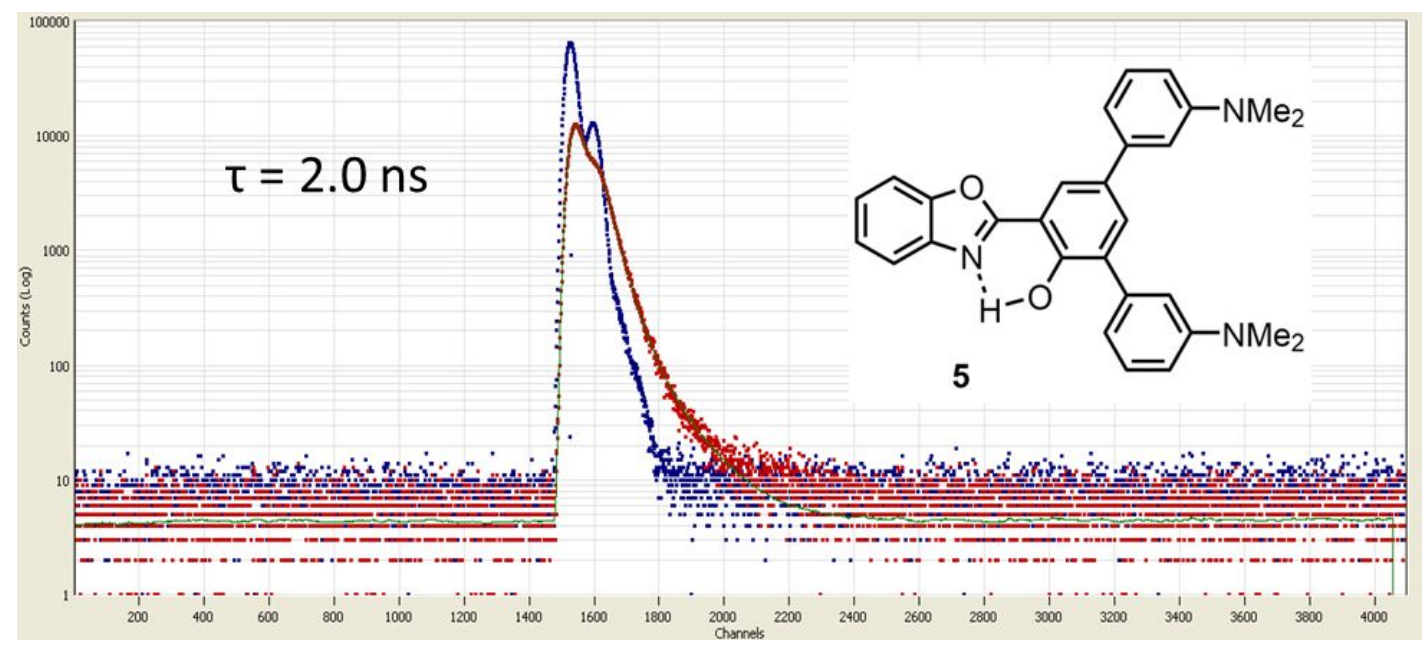

Figure S100. Time-resolved Fluorescence decays curved for dye 5 in DCM

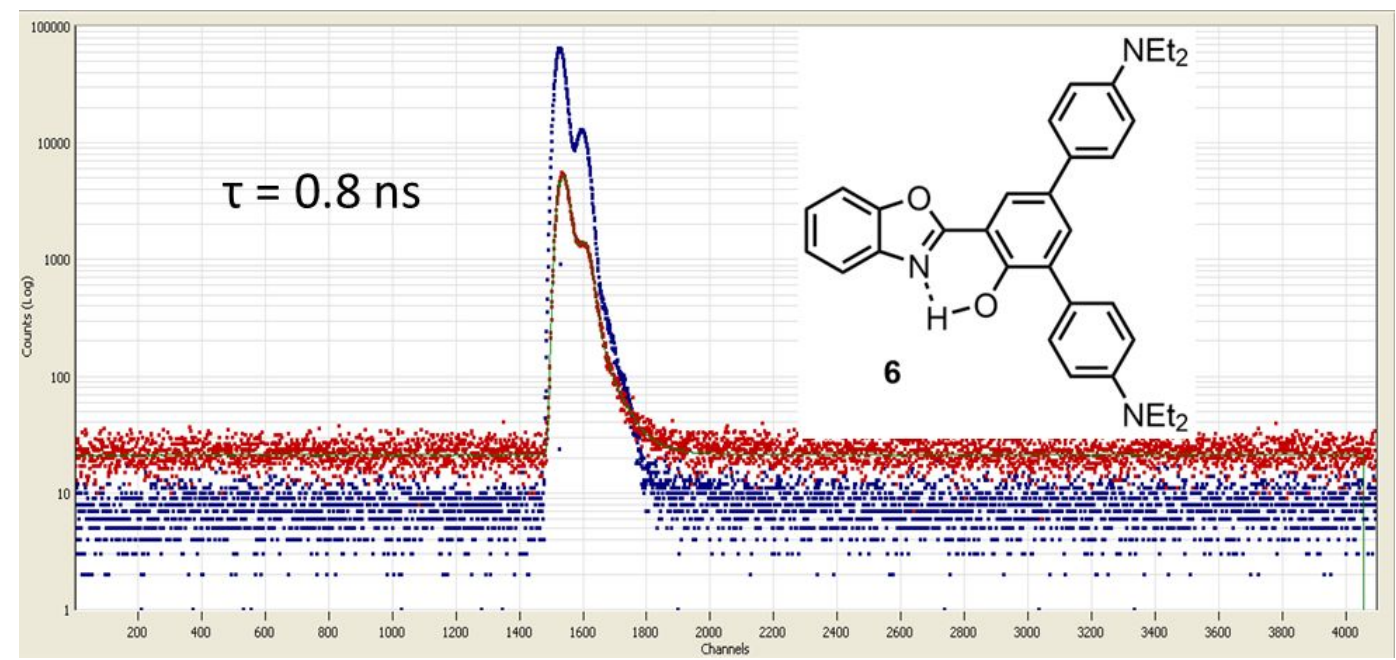

Figure S101. Time-resolved Fluorescence decays curved for dye 6 in DCM. 


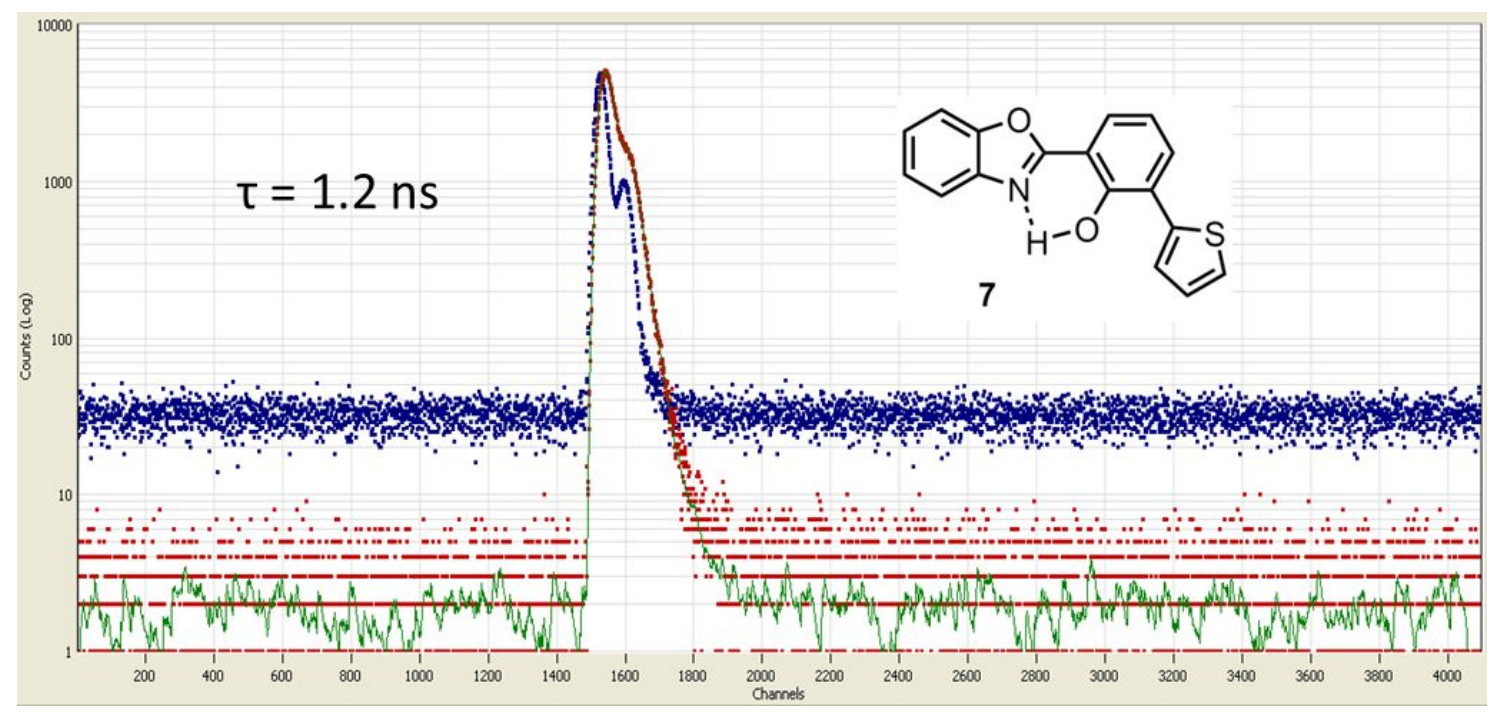

Figure S102. Time-resolved Fluorescence decays curved for dye $\mathbf{7}$ in DCM.

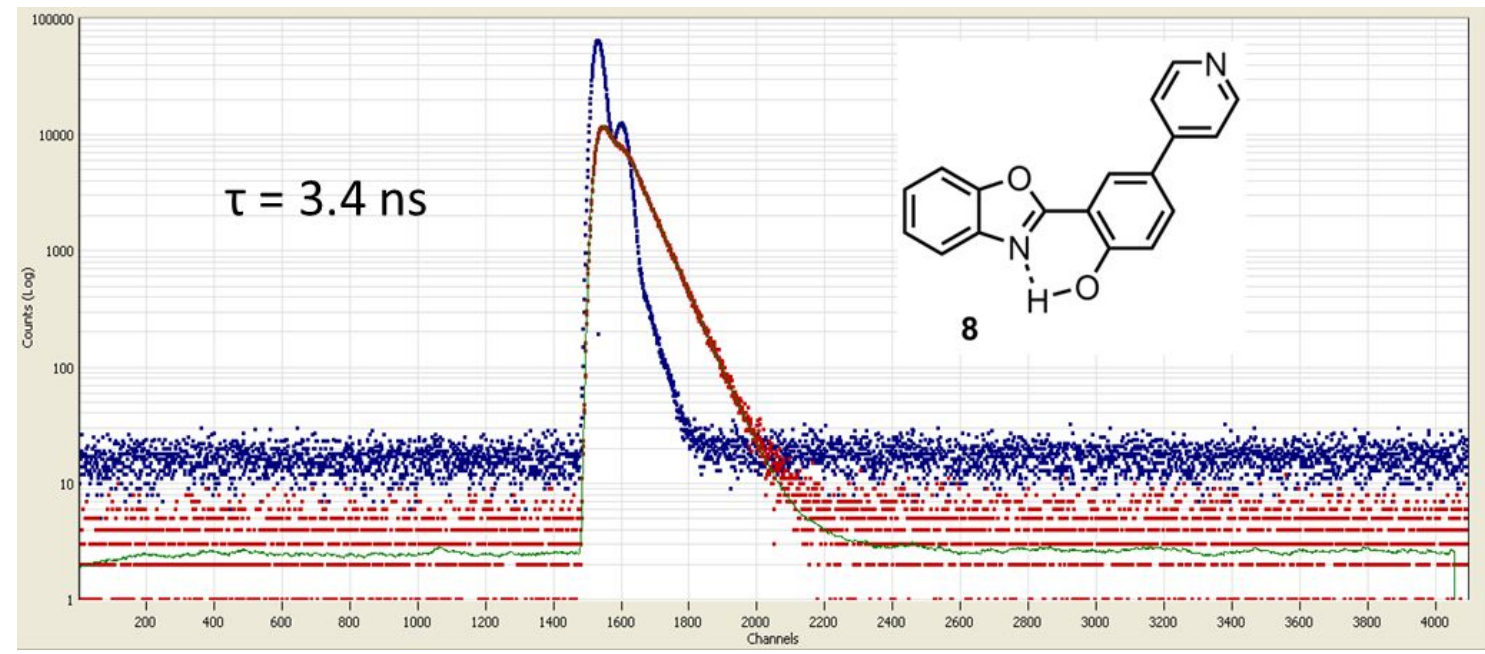

Figure S103. Time-resolved Fluorescence decays curved for dye 8 in DCM.

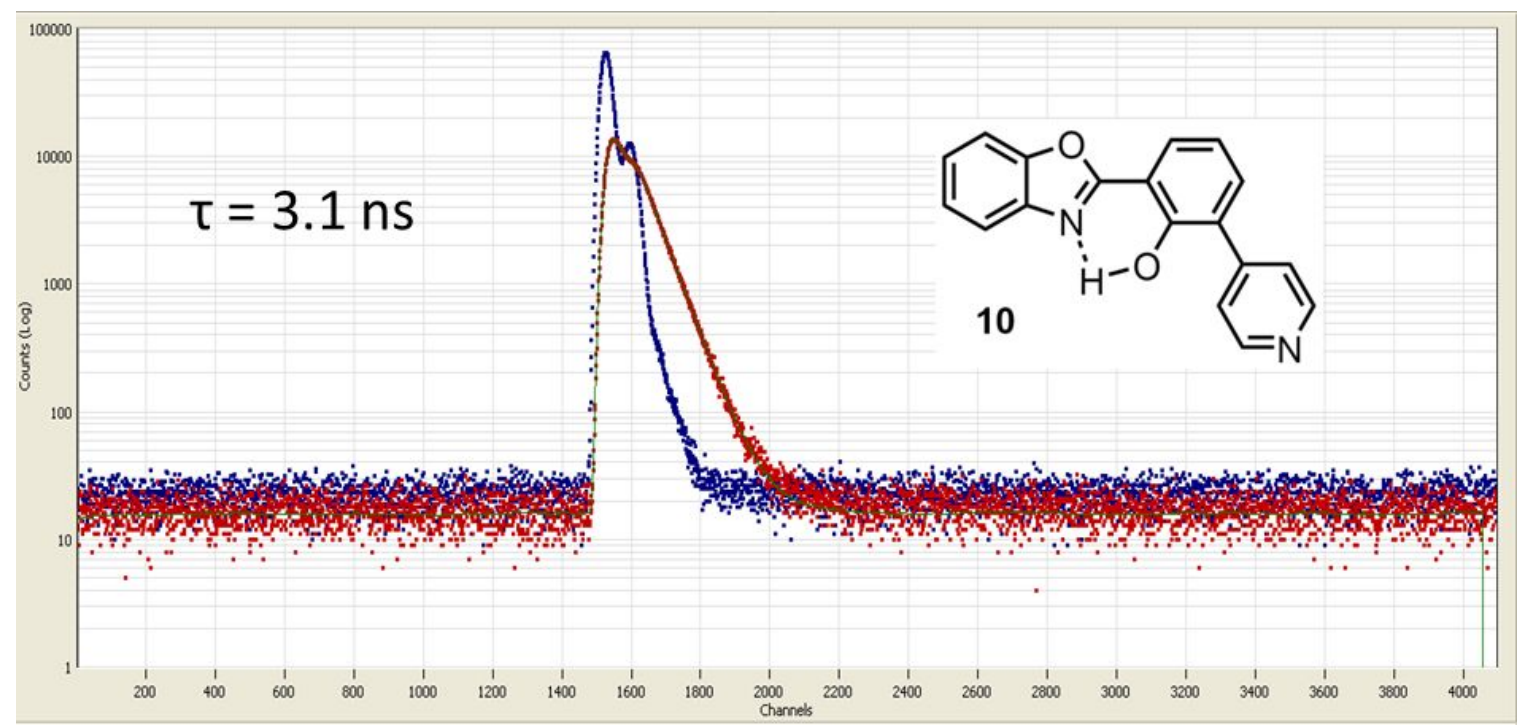

Figure S104. Time-resolved Fluorescence decays curved for dye 10 in DCM. 


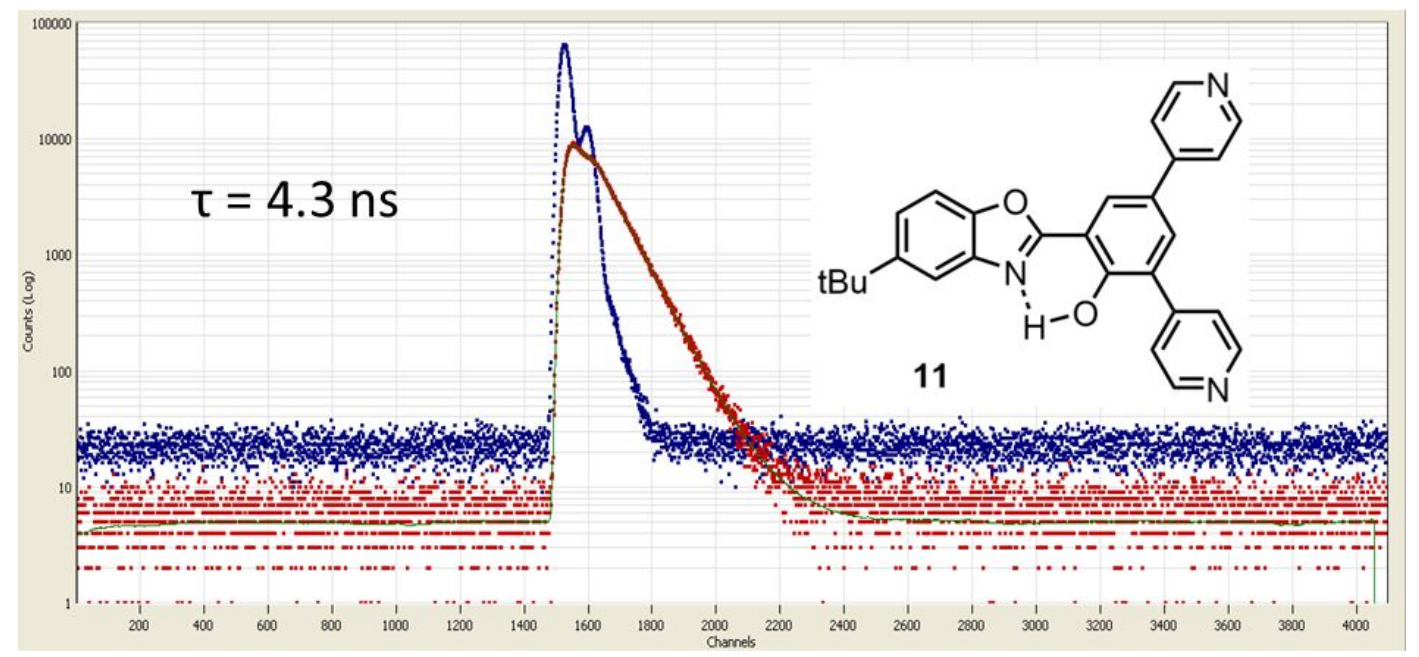

Figure S105. Time-resolved Fluorescence decays curved for dye $\mathbf{1 1}$ in DCM

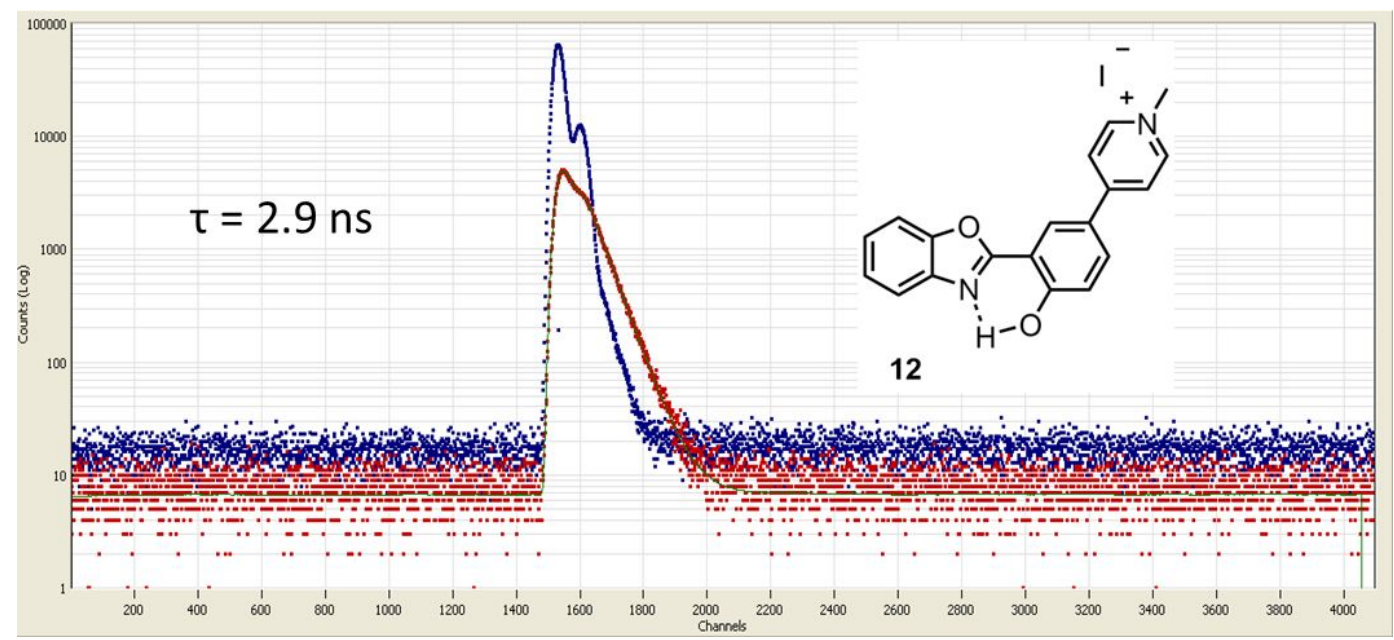

Figure S106. Time-resolved Fluorescence decays curved for dye 12 in DCM

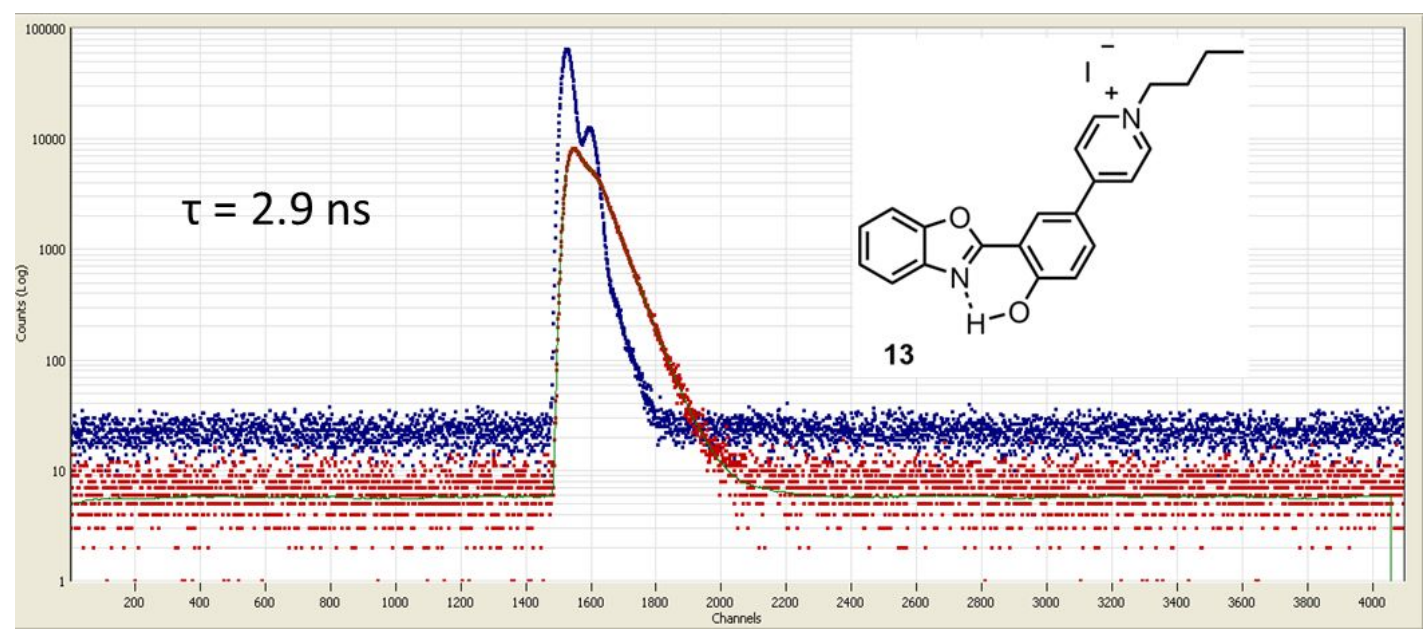

Figure S107. Time-resolved Fluorescence decays curved for dye $\mathbf{1 3}$ in DCM. 


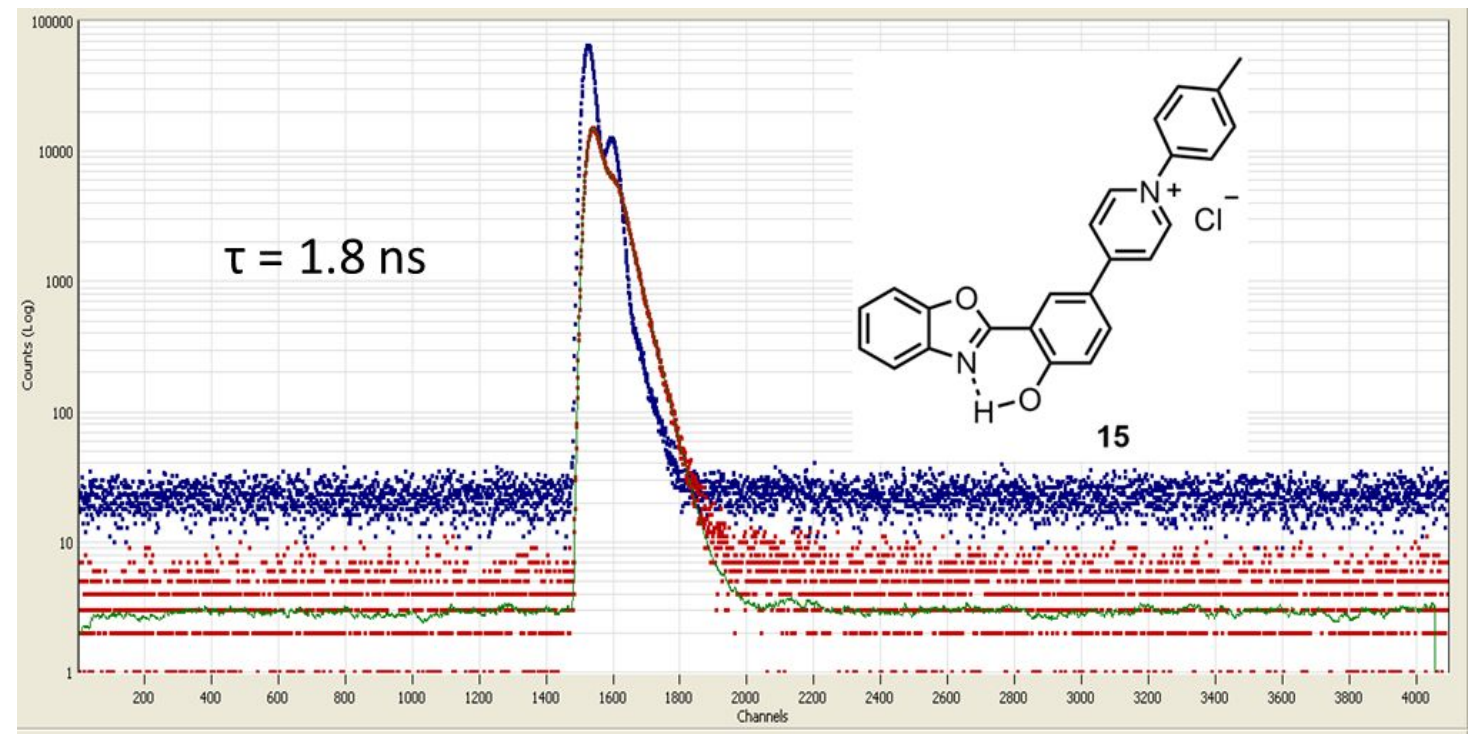

Figure S108. Time-resolved Fluorescence decays curved for dye 15 in DCM.

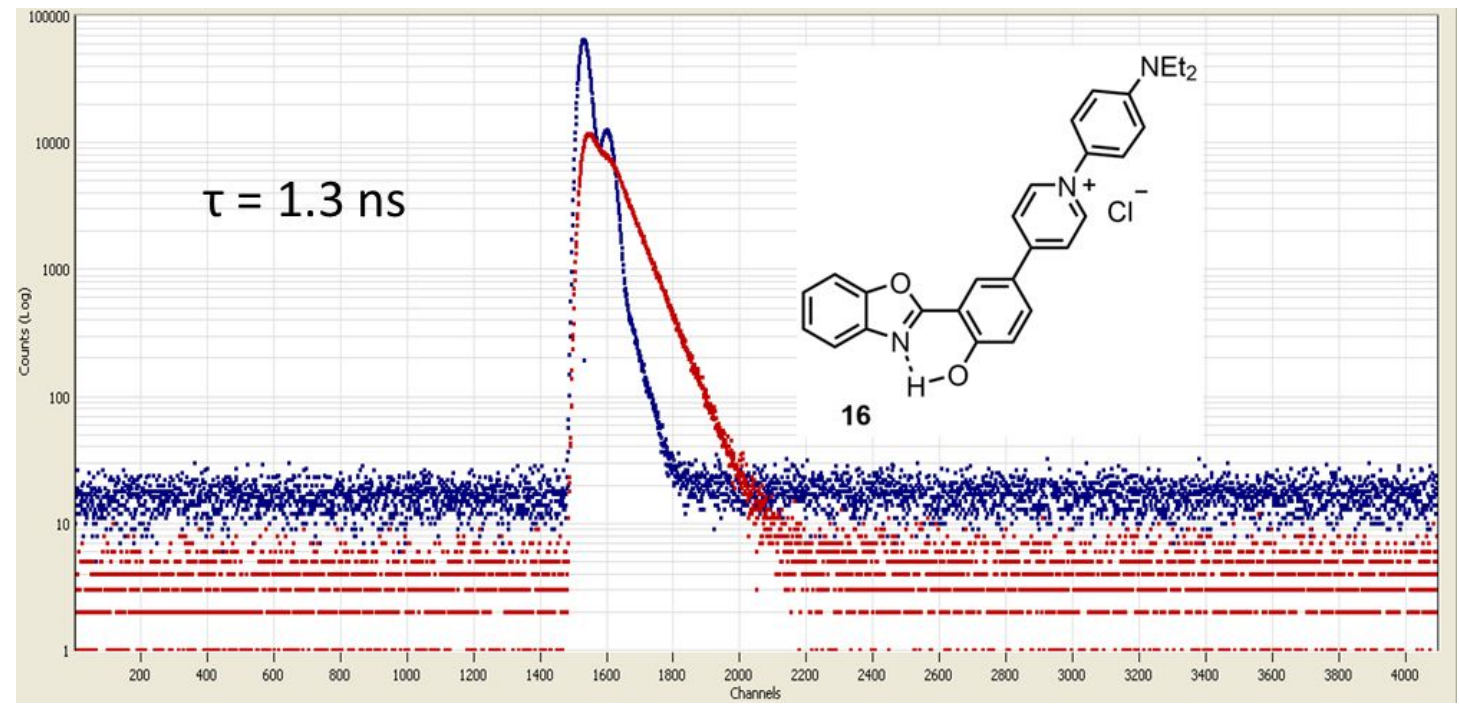

Figure S109. Time-resolved Fluorescence decays curved for dye 16 in DCM. 


\section{S4 Additional theoretical data}

Table S3. Computed relative ground-state free energies of the keto $(\mathrm{K})$ tautomer, enol-keto transition state (TS), and "inverse" (I) enol (with a $\mathrm{OH} \cdots \mathrm{O}$ instead of a $\mathrm{NH} \cdots \mathrm{O}$ bonding) as compared to the "standard" enol form. All values are in eV and have been computed in toluene. The transition state is below the keto form due to vibrational corrections which is clearly indicative of barrierless transfer from the keto to the enol in the ground-electronic state.

\begin{tabular}{|c|cccc|}
\hline HBO & $\begin{array}{c}\Delta \mathbf{G}^{\text {K-E }} \\
(\mathbf{e V})\end{array}$ & $\begin{array}{c}\Delta \mathbf{G}^{\text {TS-E }} \\
(\mathbf{e V})\end{array}$ & $\begin{array}{c}\Delta \mathbf{G}^{\text {I-E }} \\
(\mathbf{e V})\end{array}$ & Solvent \\
\hline $\mathbf{1}$ & 0.55 & 0.51 & 0.23 & toluene \\
$\mathbf{2}$ & 0.55 & 0.50 & 0.22 & toluene \\
$\mathbf{3}$ & 0.59 & 0.55 & 0.22 & toluene \\
$\mathbf{4}$ & 0.55 & 0.52 & 0.23 & toluene \\
$\mathbf{5}$ & 0.54 & 0.50 & 0.22 & toluene \\
$\mathbf{6}$ & 0.56 & 0.52 & 0.23 & toluene \\
$\mathbf{7}$ & 0.54 & 0.45 & 0.24 & toluene \\
$\mathbf{8}$ & 0.54 & 0.49 & 0.21 & toluene \\
\hline
\end{tabular}

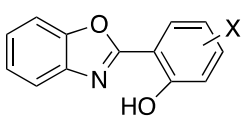

Enol (E)

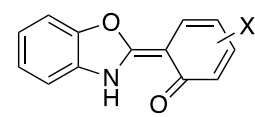

Keto (K)

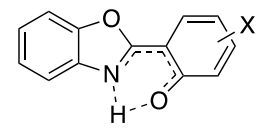

Transition state (TS)

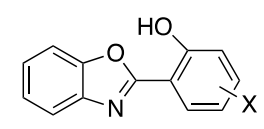

Enol-inverse (I)

Table S4. Computed relative excited-state free energies of the "inverse" (I) enol (with a $\mathrm{OH} \cdots \mathrm{O}$ instead of a $\mathrm{NH} \cdots \mathrm{O}$ bonding) as compared to the "standard" enol form. All values are in eV and have been computed in toluene.

\begin{tabular}{|c|cc|}
\hline $\mathbf{H B O}$ & $\begin{array}{c}\Delta \mathbf{G}^{*}{ }^{*} \mathrm{E}^{*} \\
(\mathbf{e V})\end{array}$ & Solvent \\
\hline $\mathbf{1}$ & 0.36 & toluene \\
$\mathbf{2}$ & 0.36 & toluene \\
$\mathbf{3}$ & 0.28 & toluene \\
$\mathbf{4}$ & 0.41 & toluene \\
$\mathbf{5}$ & 036 & toluene \\
$\mathbf{6}$ & 0.33 & toluene \\
$\mathbf{7}$ & 0.39 & toluene \\
$\mathbf{8}$ & 0.31 & toluene \\
\hline
\end{tabular}




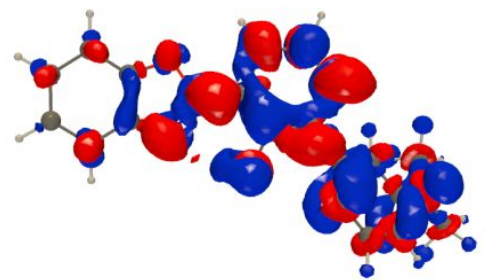

1

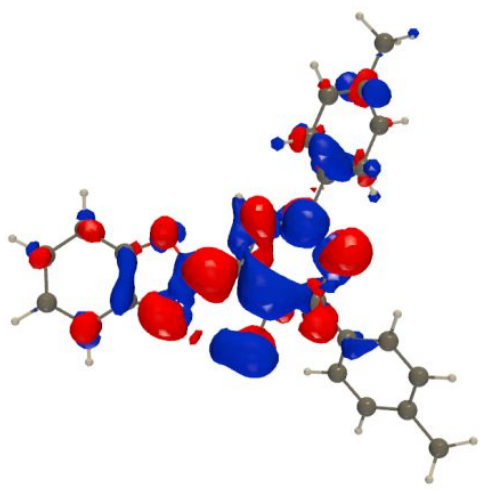

4

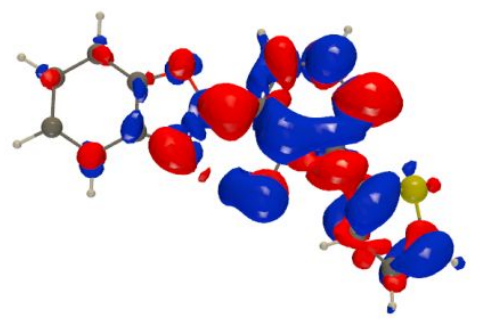

7

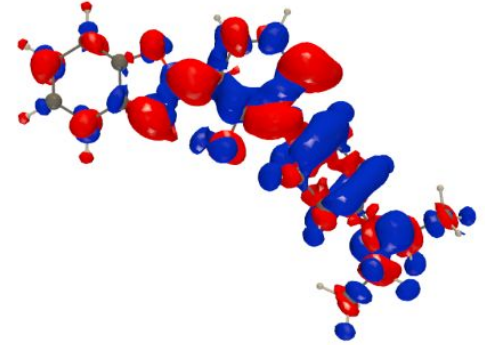

2

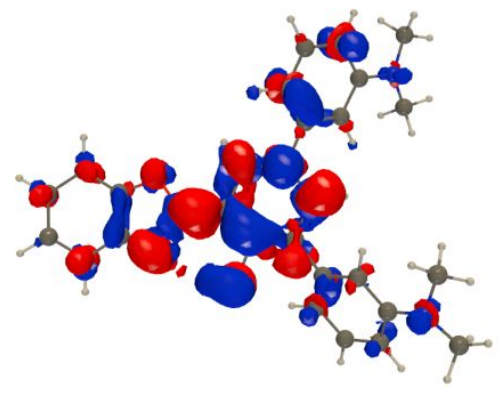

5

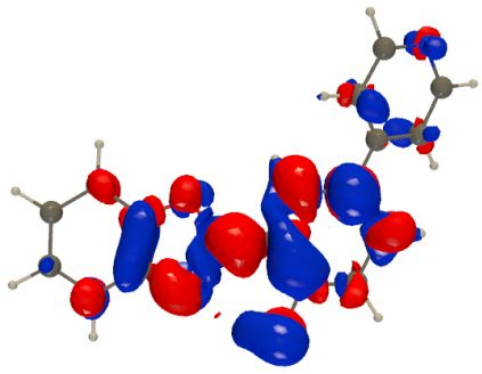

8

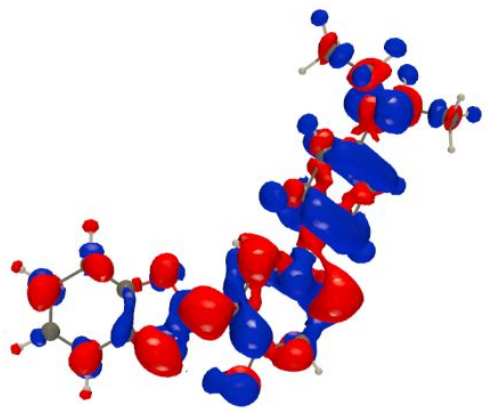

3

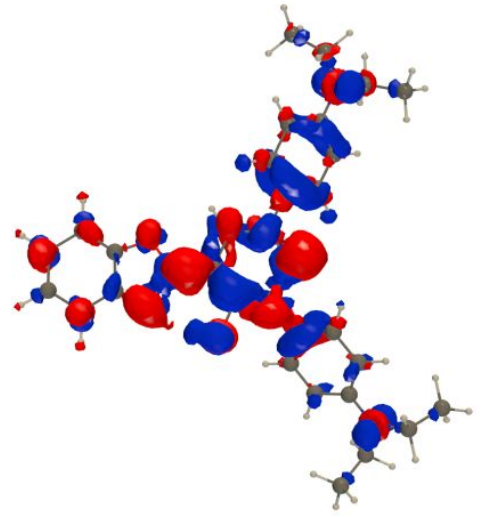

6

Figure S105. Density difference plots for the lowest excited state $\left(S_{0}-S_{1}\right.$ transition) for HBO dyes 1-8. Blue and red lobes correspond to decrease and increase of density upon absorption. Contour threshold: $1 \times 10^{-3}$ au, solvent: toluene. 


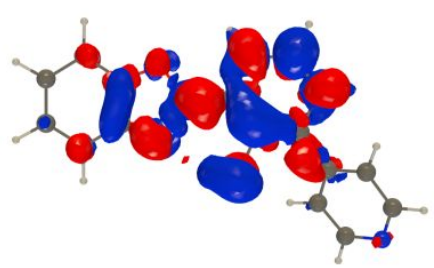

10

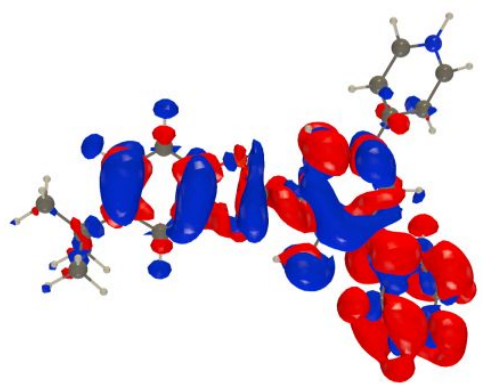

$11.2 \mathrm{H}+$

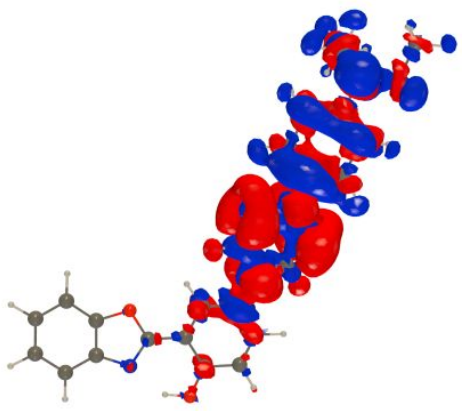

16

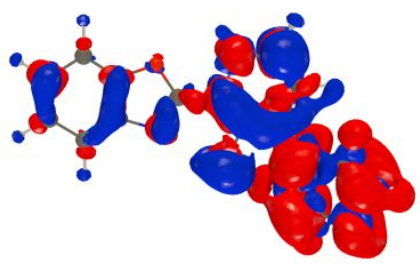

10. $\mathrm{H}^{+}$

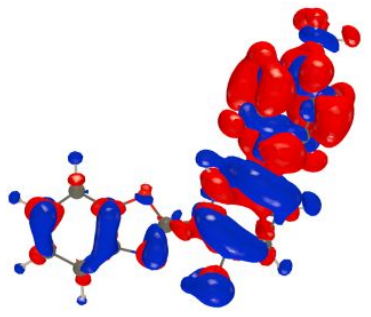

12

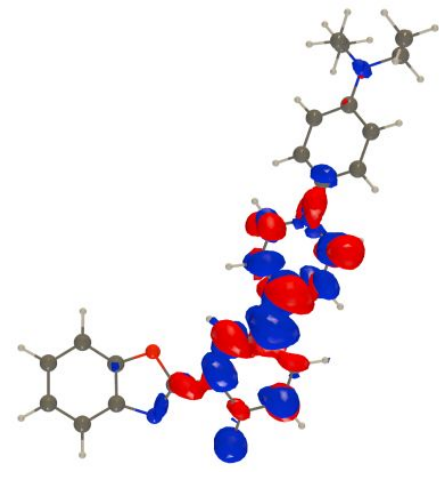

$16-\mathrm{H}^{+}$

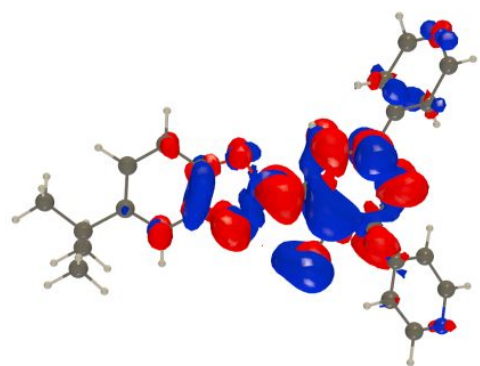

11

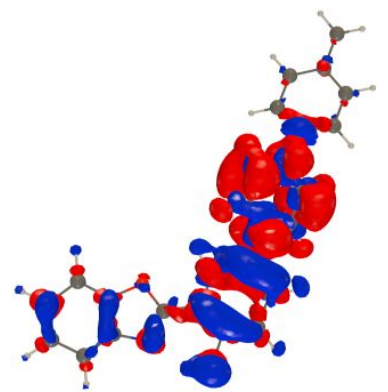

15

Figure S106. Density difference plots for the lowest excited state ( $S_{0}-S_{1}$ transition) for HBO dyes 1012, 15 and 16. Blue and red lobes correspond to decrease and increase of density upon absorption. Contour threshold: $1 \times 10^{-3}$ au, solvent: DCM. 

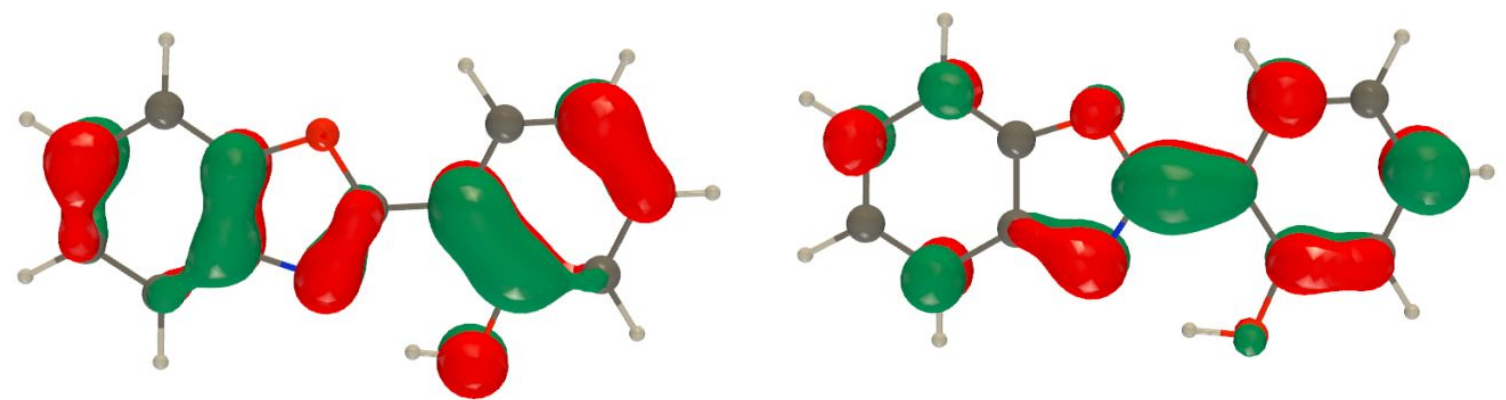

Figure S107. HOMO (left) and LUMO (right) of the unsubstituted HBO core. Note the difference between the two MOs in terms of contributions on the 3 and 5 positions of the phenol ring. 


\section{Cartesian coordinates.}

Below are given the Cartesian ( $\AA$ ) coordinates for the first series of structures as obtained at PCM(Toluene)-M06-2X/6-31G(d) level of theory. Except when noted, all structures are true minima, with no imaginary frequency. For each structure, we provide the free energy as obtained with the same level of theory.

1 (ground-state/enol) G= -1070.306658 au

\begin{tabular}{|c|c|c|c|}
\hline C & 4.2476080 & -0.9168260 & 0.2825380 \\
\hline 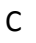 & 4.7444640 & 0.3830020 & 0.1765800 \\
\hline$C$ & 6.0844020 & 0.7093590 & 0.2925320 \\
\hline C & 6.9434910 & -0.3611010 & 0.5290860 \\
\hline C & 6.4682890 & -1.6782340 & 0.6402070 \\
\hline C & 5.1166050 & -1.9807180 & 0.5195520 \\
\hline C & 2.5979270 & 0.3941620 & -0.0768420 \\
\hline $\mathrm{H}$ & 4.7455070 & -2.9958880 & 0.6046990 \\
\hline$N$ & 2.8669780 & -0.8644280 & 0.1134750 \\
\hline $\mathrm{H}$ & 6.4363170 & 1.7304530 & 0.2045600 \\
\hline 0 & 3.6818660 & 1.2147660 & -0.0530460 \\
\hline $\mathrm{H}$ & 7.1769340 & -2.4787020 & 0.8250830 \\
\hline C & 1.2882470 & 0.9804220 & -0.3028690 \\
\hline C & 0.1510350 & 0.1423620 & -0.3332750 \\
\hline C & 1.1555310 & 2.3649660 & -0.4797070 \\
\hline C & -1.1243570 & 0.7104060 & -0.5425880 \\
\hline C & -0.0925830 & 2.9220190 & -0.6834180 \\
\hline $\mathrm{H}$ & 2.0460230 & 2.9840670 & -0.4553130 \\
\hline C & -1.2143120 & 2.0905780 & -0.7122710 \\
\hline $\mathrm{O}$ & 0.2331630 & -1.1849500 & -0.1534430 \\
\hline $\mathrm{H}$ & 1.1742360 & -1.4384610 & -0.0027810 \\
\hline $\mathrm{H}$ & 8.0068790 & -0.1715430 & 0.6299620 \\
\hline $\mathrm{H}$ & -2.1962330 & 2.5199470 & -0.8906550 \\
\hline $\mathrm{H}$ & -0.2006130 & 3.9912230 & -0.8273240 \\
\hline C & -2.3549990 & -0.1246700 & -0.5702770 \\
\hline C & -3.4839930 & 0.3209150 & 0.1208360 \\
\hline C & -2.4146460 & -1.3140170 & -1.3031370 \\
\hline C & -4.6982590 & -0.3905820 & 0.0910240 \\
\hline $\mathrm{H}$ & -3.3980380 & 1.2278480 & 0.7077940 \\
\hline C & -3.6057890 & -2.0288660 & -1.3300840 \\
\hline $\mathrm{H}$ & -1.5414720 & -1.6685930 & -1.8373930 \\
\hline C & -4.7332810 & -1.5897210 & -0.6463120 \\
\hline $\mathrm{H}$ & -3.6631970 & -2.9552540 & -1.8943700 \\
\hline $\mathrm{H}$ & -5.6365230 & -2.1860110 & -0.6853310 \\
\hline$N$ & -5.8215470 & 0.0778220 & 0.7606950 \\
\hline C & -6.9306200 & -0.8350170 & 0.9567860 \\
\hline $\mathrm{H}$ & -7.7093010 & -0.3283570 & 1.5280260 \\
\hline $\mathrm{H}$ & -7.3648540 & -1.1289930 & -0.0039290 \\
\hline $\mathrm{H}$ & -6.6382520 & -1.7490110 & 1.4956780 \\
\hline C & -5.6494640 & 1.1389500 & 1.7329920 \\
\hline $\mathrm{H}$ & -5.2876140 & 2.0516740 & 1.2491000 \\
\hline $\mathrm{H}$ & -6.6164670 & 1.3668010 & 2.1828190 \\
\hline $\mathrm{H}$ & -4.9433690 & 0.8721790 & 2.5339810 \\
\hline
\end{tabular}

1 (ground-state/keto) G=-1070.286541 au

$\begin{array}{llll}\text { C } & 4.2331310 & -0.9186730 & 0.2593680\end{array}$ 


\begin{tabular}{|c|c|c|c|}
\hline C & 4.7434700 & 0.3754850 & 0.1787570 \\
\hline C & 6.0859460 & 0.6697420 & 0.2987200 \\
\hline C & 6.9288450 & -0.4227880 & 0.5105240 \\
\hline C & 6.4302300 & -1.7282760 & 0.5936720 \\
\hline C & 5.0696110 & -2.0061930 & 0.4697680 \\
\hline C & 2.5718640 & 0.5161050 & -0.0815450 \\
\hline $\mathrm{H}$ & 4.6822930 & -3.0161970 & 0.5338750 \\
\hline N & 2.8619950 & -0.7779930 & 0.0889750 \\
\hline $\mathrm{H}$ & 6.4540530 & 1.6864350 & 0.2318030 \\
\hline 0 & 3.6989400 & 1.2507140 & -0.0324250 \\
\hline $\mathrm{H}$ & 7.1207140 & -2.5481270 & 0.7592640 \\
\hline C & 1.2954670 & 1.0591820 & -0.2827410 \\
\hline C & 0.1837020 & 0.1208110 & -0.3071090 \\
\hline C & 1.1218900 & 2.4583950 & -0.4504700 \\
\hline C & -1.1331240 & 0.7164830 & -0.5053240 \\
\hline C & -0.1310310 & 2.9654130 & -0.6408170 \\
\hline $\mathrm{H}$ & 1.9952310 & 3.1039280 & -0.4294580 \\
\hline C & -1.2405450 & 2.0779490 & -0.6656580 \\
\hline $\mathrm{O}$ & 0.3521700 & -1.1192800 & -0.1571350 \\
\hline $\mathrm{H}$ & 7.9950420 & -0.2534760 & 0.6126300 \\
\hline $\mathrm{H}$ & -2.2299060 & 2.4949270 & -0.8399050 \\
\hline $\mathrm{H}$ & -0.2876680 & 4.0288920 & -0.7809860 \\
\hline C & -2.3450710 & -0.1411470 & -0.5239130 \\
\hline C & -3.5107750 & 0.3135780 & 0.1003730 \\
\hline C & -2.3561530 & -1.3768290 & -1.1818320 \\
\hline C & -4.7091890 & -0.4224630 & 0.0661130 \\
\hline $\mathrm{H}$ & -3.4648090 & 1.2507000 & 0.6424430 \\
\hline C & -3.5313680 & -2.1169610 & -1.2102190 \\
\hline $\mathrm{H}$ & -1.4533730 & -1.7461360 & -1.6508790 \\
\hline C & -4.6944790 & -1.6629650 & -0.5986010 \\
\hline $\mathrm{H}$ & -3.5478980 & -3.0778510 & -1.7170710 \\
\hline $\mathrm{H}$ & -5.5833310 & -2.2808290 & -0.6341900 \\
\hline N & -5.8690830 & 0.0620760 & 0.6653620 \\
\hline C & -6.9554540 & -0.8705820 & 0.8924520 \\
\hline $\mathrm{H}$ & -7.7677590 & -0.3504970 & 1.4018690 \\
\hline $\mathrm{H}$ & -7.3489880 & -1.2447150 & -0.0576960 \\
\hline $\mathrm{H}$ & -6.6540890 & -1.7354770 & 1.5033320 \\
\hline C & -5.7468490 & 1.1691720 & 1.5928710 \\
\hline $\mathrm{H}$ & -5.3736720 & 2.0616270 & 1.0810570 \\
\hline $\mathrm{H}$ & -6.7341190 & 1.4076130 & 1.9907820 \\
\hline $\mathrm{H}$ & -5.0720020 & 0.9495820 & 2.4345570 \\
\hline $\mathrm{H}$ & 2.0503160 & -1.4230920 & 0.0683630 \\
\hline
\end{tabular}

1 (ground-state/PT TS) G= $-1070.288098 \mathrm{au}, v(\mathrm{imag})=552 i \mathrm{~cm}^{-1}$

$\begin{array}{lrrr}\text { C } & 4.1674320 & -0.9171800 & 0.2464080 \\ \text { C } & 4.7306090 & 0.3579970 & 0.1904820 \\ \text { C } & 6.0832490 & 0.5988460 & 0.3233170 \\ \text { C } & 6.8808340 & -0.5284400 & 0.5221090 \\ \text { C } & 6.3311980 & -1.8158340 & 0.5809280 \\ \text { C } & 4.9627100 & -2.0388830 & 0.4444220 \\ \text { C } & 2.5792070 & 0.5730980 & -0.0782580 \\ \text { H } & 4.5360060 & -3.0339180 & 0.4895740 \\ \text { N } & 2.8029460 & -0.7270290 & 0.0701810 \\ \text { H } & 6.4912590 & 1.6013050 & 0.2754340 \\ \text { O } & 3.7218590 & 1.2770470 & -0.0129570 \\ \text { H } & 6.9892190 & -2.6637770 & 0.7373480 \\ \text { C } & 1.2978350 & 1.1313540 & -0.2770030 \\ \text { C } & 0.2028870 & 0.1859270 & -0.3106000 \\ \text { C } & 1.1103160 & 2.5241300 & -0.4276270 \\ \text { C } & -1.1154180 & 0.7522620 & -0.5004000\end{array}$




$\begin{array}{lrrr}\text { C } & -0.1533200 & 3.0181930 & -0.6122250 \\ \text { H } & 1.9759700 & 3.1797430 & -0.3990910 \\ \text { C } & -1.2449790 & 2.1185240 & -0.6461240 \\ \text { O } & 0.3930800 & -1.0690090 & -0.1701020 \\ \text { H } & 1.8525910 & -1.2747580 & 0.0160510 \\ \text { H } & 7.9521970 & -0.4027360 & 0.6334620 \\ \text { H } & -2.2414850 & 2.5197980 & -0.8149350 \\ \text { H } & -0.3234880 & 4.0810800 & -0.7402670 \\ \text { C } & -2.3154090 & -0.1225570 & -0.5288890 \\ \text { C } & -3.4856580 & 0.3072350 & 0.1040370 \\ \text { C } & -2.3099490 & -1.3483350 & -1.2045140 \\ \text { C } & -4.6734040 & -0.4457330 & 0.0622210 \\ \text { H } & -3.4522000 & 1.2376160 & 0.6585320 \\ \mathrm{C} & -3.4743990 & -2.1050700 & -1.2406570 \\ \text { H } & -1.4031510 & -1.6975930 & -1.6814000 \\ \mathrm{C} & -4.6422870 & -1.6764660 & -0.6200110 \\ \text { H } & -3.4783440 & -3.0587700 & -1.7610760 \\ \text { H } & -5.5223560 & -2.3063490 & -0.6623580 \\ \mathrm{~N} & -5.8382630 & 0.0135290 & 0.6706520 \\ \mathrm{C} & -6.9129100 & -0.9359600 & 0.8831690 \\ \mathrm{H} & -7.7313690 & -0.4340700 & 1.4008540 \\ \mathrm{H} & -7.3020970 & -1.2999120 & -0.0727290 \\ \mathrm{H} & -6.6005800 & -1.8064900 & 1.4803050 \\ \mathrm{C} & -5.7305010 & 1.1098200 & 1.6126850 \\ \mathrm{H} & -5.3732490 & 2.0150760 & 1.1120040 \\ \mathrm{H} & -6.7200280 & 1.3270010 & 2.0170390 \\ \mathrm{H} & -5.0494590 & 0.8893860 & 2.4490850\end{array}$

1 (excited-state/enol) G= -1070.170312 au

$\begin{array}{lrrr}\text { C } & 4.2388540 & -0.8968640 & 0.2180890 \\ \text { C } & 4.7749000 & 0.3997020 & 0.0906270 \\ \text { C } & 6.1230830 & 0.6823090 & 0.1358460 \\ \text { C } & 6.9771200 & -0.4135990 & 0.3210420 \\ \text { C } & 6.4713540 & -1.7121920 & 0.4512390 \\ \text { C } & 5.1040270 & -1.9803400 & 0.4034610 \\ \text { C } & 2.6053550 & 0.4872090 & -0.0481580 \\ \text { H } & 4.7163970 & -2.9878630 & 0.5053010 \\ \text { N } & 2.8737090 & -0.8122190 & 0.1283290 \\ \text { H } & 6.4947530 & 1.6954400 & 0.0336160 \\ \text { O } & 3.7350800 & 1.2732500 & -0.0765070 \\ \text { H } & 7.1651430 & -2.5349830 & 0.5939770 \\ \mathrm{C} & 1.3336490 & 1.0720580 & -0.1987510 \\ \mathrm{C} & 0.1689410 & 0.2024930 & -0.1716740 \\ \mathrm{C} & 1.1200870 & 2.4584880 & -0.3454310 \\ \mathrm{C} & -1.1359330 & 0.7209290 & -0.3464000 \\ \mathrm{C} & -0.1756450 & 2.9621280 & -0.4897860 \\ \mathrm{H} & 1.9753340 & 3.1244920 & -0.3410800 \\ \mathrm{C} & -1.2922370 & 2.1331020 & -0.4813260 \\ \mathrm{O} & 0.3281190 & -1.0857810 & 0.0820940 \\ \mathrm{H} & 1.3231380 & -1.2957860 & 0.1462200 \\ \mathrm{H} & 8.0485300 & -0.2492670 & 0.3643080 \\ \mathrm{H} & -2.2781310 & 2.5407870 & -0.6683480 \\ \mathrm{H} & -0.3084970 & 4.0310630 & -0.6293520 \\ \mathrm{C} & -2.3021140 & -0.1537720 & -0.3485110 \\ \mathrm{C} & -3.5324100 & 0.3196700 & 0.1172820 \\ \mathrm{C} & -2.2406660 & -1.4630050 & -0.9046410 \\ \mathrm{C} & -4.6918210 & -0.4773900 & 0.0772210 \\ \mathrm{H} & -3.5663530 & 1.3038620 & 0.5649440 \\ \mathrm{C} & -3.3824820 & -2.2556850 & -0.9505300 \\ \mathrm{H} & -1.3108460 & -1.8242760 & -1.3216670\end{array}$




$\begin{array}{llll}\mathrm{C} & -4.5955390 & -1.7897080 & -0.4712150 \\ \mathrm{H} & -3.3286000 & -3.2497020 & -1.3819690 \\ \mathrm{H} & -5.4708470 & -2.4246030 & -0.5288890 \\ \mathrm{~N} & -5.8849120 & -0.0188830 & 0.5551490 \\ \mathrm{C} & -7.0558090 & -0.8789860 & 0.5754850 \\ \mathrm{H} & -7.8828490 & -0.3380520 & 1.0324090 \\ \mathrm{H} & -7.3543720 & -1.1705220 & -0.4378110 \\ \mathrm{H} & -6.8724880 & -1.7863620 & 1.1614800 \\ \mathrm{C} & -5.9741770 & 1.3247730 & 1.0947470 \\ \mathrm{H} & -5.6601570 & 2.0645890 & 0.3497780 \\ \mathrm{H} & -7.0064890 & 1.5306050 & 1.3727500 \\ \mathrm{H} & -5.3420750 & 1.4406130 & 1.9835820\end{array}$

1 (excited-state/keto) G= -1070.179294 au

$\begin{array}{lrrr}\text { C } & 4.3460440 & -0.8977200 & 0.2379170 \\ \text { C } & 4.7966410 & 0.4199390 & 0.0789890 \\ \text { C } & 6.1298780 & 0.7678690 & 0.0963910 \\ \text { C } & 7.0378010 & -0.2829340 & 0.2861340 \\ \text { C } & 6.6024550 & -1.6004410 & 0.4467070 \\ \text { C } & 5.2476060 & -1.9409070 & 0.4269070 \\ \text { C } & 2.5963430 & 0.4664840 & -0.0362940 \\ \text { H } & 4.9129080 & -2.9638880 & 0.5519200 \\ \text { N } & 2.9745510 & -0.8235900 & 0.1621370 \\ \text { H } & 6.4473980 & 1.7959600 & -0.0299460 \\ \text { O } & 3.7140060 & 1.2452850 & -0.0866080 \\ \text { H } & 7.3368130 & -2.3858910 & 0.5915070 \\ \text { C } & 1.3024290 & 1.0124450 & -0.1881950 \\ \text { C } & 0.1464800 & 0.0851490 & -0.1633680 \\ \text { C } & 1.1044080 & 2.3785940 & -0.3207340 \\ \text { C } & -1.1699400 & 0.6527520 & -0.3478650 \\ \text { C } & -0.2041870 & 2.8971060 & -0.4941910 \\ \text { H } & 1.9552430 & 3.0518420 & -0.2947700 \\ \text { C } & -1.3031390 & 2.0644480 & -0.5007450 \\ \text { O } & 0.3582480 & -1.1344750 & 0.0533180 \\ \text { H } & 8.0998460 & -0.0649670 & 0.3084360 \\ \text { H } & -2.2861410 & 2.4807600 & -0.6906960 \\ \text { H } & -0.3297590 & 3.9652210 & -0.6388940 \\ \text { C } & -2.3527650 & -0.2008910 & -0.3726930 \\ \text { C } & -3.5933430 & 0.3203500 & 0.0450790 \\ \text { C } & -2.3030840 & -1.5308440 & -0.8438780 \\ \text { C } & -4.7732570 & -0.4365980 & 0.0052450 \\ \text { H } & -3.6138250 & 1.3207930 & 0.4571070 \\ \text { C } & -3.4658710 & -2.2849190 & -0.8796060 \\ \text { H } & -1.3652230 & -1.9443610 & -1.1867570 \\ \text { C } & -4.6868730 & -1.7665080 & -0.4610260 \\ \text { H } & -3.4293600 & -3.3065800 & -1.2467580 \\ \text { H } & -5.5678670 & -2.3953730 & -0.4994320 \\ \text { N } & -5.9891660 & 0.0997170 & 0.4063840 \\ \text { C } & -7.0906640 & -0.8063150 & 0.6690960 \\ \text { H } & -7.9459720 & -0.2293050 & 1.0222350 \\ \text { H } & -7.3973940 & -1.3227170 & -0.2460260 \\ \text { H } & -6.8435190 & -1.5652100 & 1.4263600 \\ \text { C } & -5.9855670 & 1.3714080 & 1.1001220 \\ \text { H } & -5.5732970 & 2.1573970 & 0.4592270 \\ \text { H } & -7.0129030 & 1.6465980 & 1.3412810 \\ \text { H } & -5.4000840 & 1.3469860 & 2.0319610 \\ \text { H } & 2.2490290 & -1.5374400 & 0.2259800\end{array}$

1 (excited-state/ESIPT TS) G= $-1070.170857 \mathrm{au}, v(\mathrm{imag})=1163 i \mathrm{~cm}^{-1}$

$\begin{array}{llll}\text { C } & 4.1726800 & -0.8958660 & 0.2120160\end{array}$ 


\begin{tabular}{|c|c|c|c|}
\hline C & 4.7672140 & 0.3784050 & 0.1155360 \\
\hline C & 6.1260400 & 0.5938040 & 0.1873270 \\
\hline C & 6.9227340 & -0.5454260 & 0.3670030 \\
\hline C & 6.3544810 & -1.8198200 & 0.4661380 \\
\hline C & 4.9766600 & -2.0240210 & 0.3919130 \\
\hline C & 2.6096330 & 0.6010160 & -0.0601780 \\
\hline $\mathrm{H}$ & 4.5406250 & -3.0133070 & 0.4694540 \\
\hline N & 2.8190950 & -0.7200170 & 0.0986830 \\
\hline $\mathrm{H}$ & 6.5465820 & 1.5895480 & 0.1089840 \\
\hline 0 & 3.7766300 & 1.3132300 & -0.0540990 \\
\hline $\mathrm{H}$ & 7.0054940 & -2.6770760 & 0.6055310 \\
\hline C & 1.3390060 & 1.1823960 & -0.2098300 \\
\hline C & 0.1952710 & 0.2487030 & -0.2018890 \\
\hline C & 1.0929540 & 2.5502300 & -0.3282760 \\
\hline C & -1.1346940 & 0.7479520 & -0.3666370 \\
\hline C & -0.2283750 & 3.0177800 & -0.4740780 \\
\hline $\mathrm{H}$ & 1.9242260 & 3.2464330 & -0.3073390 \\
\hline C & -1.3154870 & 2.1559900 & -0.4831340 \\
\hline $\mathrm{O}$ & 0.4274580 & -1.0047530 & 0.0053120 \\
\hline $\mathrm{H}$ & 1.6068590 & -1.1687240 & 0.0883310 \\
\hline $\mathrm{H}$ & 7.9996570 & -0.4332260 & 0.4303200 \\
\hline $\mathrm{H}$ & -2.3105320 & 2.5455460 & -0.6610570 \\
\hline $\mathrm{H}$ & -0.3926640 & 4.0837160 & -0.5988910 \\
\hline C & -2.2826110 & -0.1516630 & -0.3891550 \\
\hline C & -3.5262530 & 0.3072670 & 0.0792050 \\
\hline C & -2.1943730 & -1.4561570 & -0.9287040 \\
\hline C & -4.6768430 & -0.4947450 & 0.0352420 \\
\hline $\mathrm{H}$ & -3.5704220 & 1.2888580 & 0.5326300 \\
\hline C & -3.3282940 & -2.2536070 & -0.9731740 \\
\hline $\mathrm{H}$ & -1.2551700 & -1.8150520 & -1.3262550 \\
\hline C & -4.5534570 & -1.8002600 & -0.4991710 \\
\hline $\mathrm{H}$ & -3.2643150 & -3.2530370 & -1.3924750 \\
\hline $\mathrm{H}$ & -5.4137710 & -2.4565870 & -0.5472750 \\
\hline N & -5.8920430 & -0.0344540 & 0.4961860 \\
\hline C & -6.9907070 & -0.9657160 & 0.6636860 \\
\hline $\mathrm{H}$ & -7.8466610 & -0.4315270 & 1.0759320 \\
\hline $\mathrm{H}$ & -7.2962340 & -1.3917770 & -0.2980370 \\
\hline $\mathrm{H}$ & -6.7370250 & -1.7919440 & 1.3423270 \\
\hline C & -5.9487440 & 1.2443920 & 1.1730270 \\
\hline $\mathrm{H}$ & -5.6019300 & 2.0466790 & 0.5126610 \\
\hline $\mathrm{H}$ & -6.9824780 & 1.4579970 & 1.4445950 \\
\hline $\mathrm{H}$ & -5.3353360 & 1.2630340 & 2.0855710 \\
\hline
\end{tabular}


2 (ground-state) G= $-1148.843504 \mathrm{au}$

\begin{tabular}{|c|c|c|c|}
\hline$C$ & 4.7528590 & -1.2020840 & 0.1499190 \\
\hline C & 5.4683070 & -0.0092670 & 0.0356210 \\
\hline C & 6.8501440 & 0.0664720 & 0.0460470 \\
\hline C & 7.5161500 & -1.1493360 & 0.1812010 \\
\hline C & 6.8192950 & -2.3632530 & 0.2981280 \\
\hline C & 5.4297690 & -2.4131700 & 0.2847660 \\
\hline C & 3.3462400 & 0.3973630 & -0.0347960 \\
\hline $\mathrm{H}$ & 4.8880240 & -3.3480420 & 0.3748670 \\
\hline $\mathrm{N}$ & 3.3959410 & -0.8960040 & 0.0996910 \\
\hline $\mathrm{H}$ & 7.3738730 & 1.0107530 & -0.0449740 \\
\hline 0 & 4.5605580 & 1.0081010 & -0.0819570 \\
\hline $\mathrm{H}$ & 7.3826670 & -3.2847150 & 0.4014760 \\
\hline C & 2.1526790 & 1.2191330 & -0.1333680 \\
\hline C & 0.8825430 & 0.6037160 & -0.0843930 \\
\hline C & 2.2643200 & 2.6113620 & -0.2628230 \\
\hline C & -0.2830520 & 1.3980230 & -0.1692480 \\
\hline C & 1.1266330 & 3.3907830 & -0.3449350 \\
\hline $\mathrm{H}$ & 3.2523170 & 3.0576060 & -0.2997830 \\
\hline C & -0.1274090 & 2.7775130 & -0.3007960 \\
\hline 0 & 0.7346170 & -0.7242160 & 0.0556220 \\
\hline $\mathrm{H}$ & 1.6222550 & -1.1485090 & 0.1201110 \\
\hline $\mathrm{H}$ & 8.6008760 & -1.1576250 & 0.1965380 \\
\hline $\mathrm{H}$ & -1.0228330 & 3.3863670 & -0.3892060 \\
\hline $\mathrm{H}$ & 1.2044210 & 4.4669200 & -0.4531260 \\
\hline C & -1.6390270 & 0.8037040 & -0.0968700 \\
\hline C & -2.6491650 & 1.4367030 & 0.6349550 \\
\hline C & -1.9896470 & -0.3693470 & -0.7762300 \\
\hline C & -3.9435440 & 0.9411710 & 0.6886650 \\
\hline $\mathrm{H}$ & -2.4157170 & 2.3404680 & 1.1924600 \\
\hline C & -3.2773600 & -0.8800400 & -0.7337430 \\
\hline $\mathrm{H}$ & -1.2364350 & -0.8978260 & -1.3501870 \\
\hline C & -4.2987340 & -0.2390980 & 0.0016800 \\
\hline $\mathrm{H}$ & -4.6819380 & 1.4854040 & 1.2655350 \\
\hline $\mathrm{H}$ & -3.4832330 & -1.7992330 & -1.2693890 \\
\hline $\mathrm{N}$ & -5.5810990 & -0.7445630 & 0.0486650 \\
\hline C & -5.9807790 & -1.8817610 & -0.7619170 \\
\hline C & -5.6819730 & -3.2260450 & -0.0984900 \\
\hline $\mathrm{H}$ & -5.4988720 & -1.8162400 & -1.7426090 \\
\hline $\mathrm{H}$ & -7.0565670 & -1.7904210 & -0.9481670 \\
\hline $\mathrm{H}$ & -6.0007850 & -4.0556750 & -0.7367630 \\
\hline $\mathrm{H}$ & -4.6118840 & -3.3316180 & 0.1014510 \\
\hline $\mathrm{H}$ & -6.2108280 & -3.3042390 & 0.8565880 \\
\hline C & -6.5898400 & -0.1765270 & 0.9258020 \\
\hline C & -7.3415030 & 0.9949900 & 0.2940760 \\
\hline $\mathrm{H}$ & -6.1238940 & 0.1239340 & 1.8698820 \\
\hline $\mathrm{H}$ & -7.2945100 & -0.9764070 & 1.1786700 \\
\hline $\mathrm{H}$ & -8.0932500 & 1.3935120 & 0.9821010 \\
\hline $\mathrm{H}$ & -6.6514820 & 1.8006820 & 0.0274570 \\
\hline $\mathrm{H}$ & -7.8489240 & 0.6717830 & -0.6202600 \\
\hline
\end{tabular}

2 (ground-state/keto) G= $-1148.823374 \mathrm{au}$

$\begin{array}{lrrr}\text { C } & 4.7012540 & -1.2204970 & 0.1226720 \\ \text { C } & 5.4437390 & -0.0437980 & 0.0443850 \\ \text { C } & 6.8226260 & -0.0142040 & 0.0693650 \\ \text { C } & 7.4537100 & -1.2546280 & 0.1803180 \\ \text { C } & 6.7210900 & -2.4446460 & 0.2602970 \\ \text { C } & 5.3269000 & -2.4546400 & 0.2330770 \\ \text { C } & 3.3264910 & 0.5179090 & -0.0409210 \\ \text { H } & 4.7591790 & -3.3755350 & 0.2954150\end{array}$




$\begin{array}{lrrr}\text { N } & 3.3741930 & -0.8152300 & 0.0633450 \\ \text { H } & 7.3727640 & 0.9169670 & 0.0065220 \\ \text { O } & 4.5753380 & 1.0221710 & -0.0566890 \\ \text { H } & 7.2513440 & -3.3869070 & 0.3460610 \\ \text { C } & 2.1697410 & 1.3031840 & -0.1212730 \\ \text { C } & 0.8983170 & 0.5978090 & -0.0850150 \\ \text { C } & 2.2603540 & 2.7178040 & -0.2250420 \\ \text { C } & -0.2918400 & 1.4407360 & -0.1529360 \\ \mathrm{C} & 1.1185260 & 3.4608740 & -0.2951300 \\ \text { H } & 3.2413960 & 3.1830180 & -0.2523580 \\ \mathrm{C} & -0.1417380 & 2.8037210 & -0.2617190 \\ \text { O } & 0.8331290 & -0.6584330 & 0.0071650 \\ \text { H } & 8.5370860 & -1.2942250 & 0.2051340 \\ \text { H } & -1.0396370 & 3.4121050 & -0.3432540 \\ \text { H } & 1.1602340 & 4.5405480 & -0.3850460 \\ \mathrm{C} & -1.6389310 & 0.8322640 & -0.0816050 \\ \mathrm{C} & -2.6771030 & 1.4729230 & 0.6045600 \\ \mathrm{C} & -1.9565500 & -0.3769000 & -0.7147430 \\ \mathrm{C} & -3.9654050 & 0.9599150 & 0.6484110 \\ \mathrm{H} & -2.4708910 & 2.3989980 & 1.1355730 \\ \mathrm{C} & -3.2377560 & -0.9060490 & -0.6797980 \\ \mathrm{H} & -1.1782500 & -0.9175880 & -1.2397420 \\ \mathrm{C} & -4.2865780 & -0.2517300 & 0.0017810 \\ \mathrm{H} & -4.7245280 & 1.5135660 & 1.1886580 \\ \mathrm{H} & -3.4164340 & -1.8517550 & -1.1783020 \\ \mathrm{~N} & -5.5644860 & -0.7749860 & 0.0385300 \\ \mathrm{C} & -5.9257660 & -1.9526300 & -0.7296980 \\ \mathrm{C} & -5.6231400 & -3.2618230 & -0.0005040 \\ \mathrm{H} & -5.4197030 & -1.9227500 & -1.6999520 \\ \mathrm{H} & -6.9978720 & -1.8880580 & -0.9469150 \\ \mathrm{H} & -5.9104870 & -4.1243530 & -0.6096230 \\ \mathrm{H} & -4.5572090 & -3.3387110 & 0.2320460 \\ \mathrm{H} & -6.1763060 & -3.3077260 & 0.9429140 \\ \mathrm{C} & -6.6002730 & -0.1895150 & 0.8703440 \\ \mathrm{C} & -7.3530800 & 0.9470020 & 0.1785430 \\ \mathrm{H} & -6.1606690 & 0.1534440 & 1.8127440 \\ \mathrm{H} & -7.3004580 & -0.9888540 & 1.1376320 \\ & -6.6672640 & 1.3610110 & 0.8338950 \\ & -7.8350240 & 0.58525270 & -0.7340900 \\ & & -1.2937720 & 0.0866020\end{array}$

2 (ground-state/PT TS) G= --1148.824987 au, $v\left(\right.$ imag) $=543 i \mathrm{~cm}^{-1}$

$\begin{array}{lrrr}\text { C } & 4.6199810 & -1.2174140 & 0.1201730 \\ \text { C } & 5.4178290 & -0.0749770 & 0.0492360 \\ \text { C } & 6.7973330 & -0.1073350 & 0.0803750 \\ \text { C } & 7.3698550 & -1.3749880 & 0.1899850 \\ \text { C } & 6.5832490 & -2.5318850 & 0.2626960 \\ \text { C } & 5.1912590 & -2.4789300 & 0.2292550 \\ \text { C } & 3.3366540 & 0.5654430 & -0.0430900 \\ \text { H } & 4.5814630 & -3.3729090 & 0.2854260 \\ \text { N } & 3.3107680 & -0.7589470 & 0.0561460 \\ \text { H } & 7.3900290 & 0.7977640 & 0.0231510 \\ \text { O } & 4.5980510 & 1.0299040 & -0.0526080 \\ \text { H } & 7.0710880 & -3.4969300 & 0.3474820 \\ \text { C } & 2.1807140 & 1.3699880 & -0.1240480 \\ \text { C } & 0.9212920 & 0.6595120 & -0.0886090 \\ \text { C } & 2.2621930 & 2.7789710 & -0.2228170 \\ \text { C } & -0.2727400 & 1.4770830 & -0.1532960 \\ \text { C } & 1.1102100 & 3.5145450 & -0.2901910\end{array}$




$\begin{array}{lrrr}\text { H } & 3.2397790 & 3.2516410 & -0.2488180 \\ \mathrm{C} & -0.1378610 & 2.8469360 & -0.2580350 \\ \mathrm{O} & 0.8688710 & -0.6140280 & 0.0036550 \\ \mathrm{H} & 2.2712120 & -1.1090780 & 0.0669440 \\ \mathrm{H} & 8.4502030 & -1.4644190 & 0.2196450 \\ \mathrm{H} & -1.0436770 & 3.4434880 & -0.3366730 \\ \mathrm{H} & 1.1429150 & 4.5947390 & -0.3769250 \\ \mathrm{C} & -1.6140750 & 0.8547120 & -0.0840220 \\ \mathrm{C} & -2.6567420 & 1.4803650 & 0.6087460 \\ \mathrm{C} & -1.9203910 & -0.3529500 & -0.7250120 \\ \mathrm{C} & -3.9391830 & 0.9529110 & 0.6531870 \\ \mathrm{H} & -2.4592080 & 2.4054990 & 1.1446880 \\ \mathrm{C} & -3.1954880 & -0.8963860 & -0.6896890 \\ \mathrm{H} & -1.1380740 & -0.8815580 & -1.2565920 \\ \mathrm{C} & -4.2490870 & -0.2581730 & -0.0000770 \\ \mathrm{H} & -4.7027250 & 1.4949240 & 1.1989330 \\ \mathrm{H} & -3.3654170 & -1.8405390 & -1.1940920 \\ \mathrm{~N} & -5.5207090 & -0.7958260 & 0.0376990 \\ \mathrm{C} & -5.8715150 & -1.9727440 & -0.7366570 \\ \mathrm{C} & -5.5512850 & -3.2828950 & -0.0168170 \\ \mathrm{H} & -5.3695360 & -1.9309780 & -1.7085850 \\ \mathrm{H} & -6.9450900 & -1.9188970 & -0.9494430 \\ \mathrm{H} & -5.8309400 & -4.1447750 & -0.6303970 \\ \mathrm{H} & -4.4837260 & -3.3490920 & 0.2114920 \\ \mathrm{H} & -6.1004730 & -3.3411210 & 0.9282410 \\ \mathrm{C} & -6.5601150 & -0.2272770 & 0.8768340 \\ \mathrm{C} & -7.3283000 & 0.9047330 & 0.1946310 \\ \mathrm{H} & -6.1210110 & 0.1148170 & 1.8197660 \\ \mathrm{H} & -7.2501200 & -1.0362130 & 1.1416230 \\ \mathrm{H} & -8.1033850 & 1.3054930 & 0.8550600 \\ \mathrm{H} & -6.6528930 & 1.7186800 & -0.0840280 \\ \mathrm{H} & -7.8089170 & 0.5403960 & -0.7187470\end{array}$

2 (excited-state/enol) G= $-1148.716298 \mathrm{au}$

$\begin{array}{lrrr}\text { C } & 4.6870700 & -1.2062130 & 0.0602260 \\ \text { C } & 5.4465210 & -0.0227870 & 0.0053690 \\ \text { C } & 6.8238330 & 0.0088770 & 0.0076430 \\ \text { C } & 7.4718200 & -1.2336700 & 0.0701960 \\ \text { C } & 6.7421980 & -2.4244160 & 0.1268610 \\ \text { C } & 5.3462100 & -2.4367870 & 0.1229480 \\ \text { C } & 3.3244270 & 0.4649820 & -0.0235430 \\ \text { H } & 4.7855110 & -3.3639840 & 0.1672710 \\ \text { N } & 3.3552950 & -0.8703860 & 0.0404410 \\ \text { H } & 7.3697690 & 0.9444880 & -0.0362470 \\ \text { O } & 4.5787130 & 1.0350910 & -0.0474010 \\ \text { H } & 7.2767950 & -3.3682900 & 0.1752800 \\ \text { C } & 2.1793440 & 1.2861620 & -0.0591070 \\ \text { C } & 0.8765560 & 0.6577420 & -0.0350040 \\ \text { C } & 2.2332250 & 2.6951580 & -0.0992760 \\ \text { C } & -0.3053210 & 1.4317550 & -0.0659840 \\ \text { C } & 1.0617660 & 3.4579600 & -0.1277320 \\ \text { H } & 3.2039700 & 3.1789000 & -0.1053670 \\ \text { C } & -0.1883240 & 2.8692820 & -0.0887420 \\ \text { O } & 0.7947870 & -0.6648350 & 0.0717770 \\ \text { H } & 1.7306660 & -1.0551080 & 0.0855240 \\ \text { H } & 8.5561190 & -1.2670780 & 0.0749550 \\ \text { H } & -1.0782520 & 3.4749260 & -0.2023420 \\ \text { H } & 1.1426710 & 4.5391690 & -0.1920510 \\ \text { C } & -1.6161250 & 0.8281410 & -0.0468440 \\ \text { C } & -2.7291470 & 1.5587450 & 0.4639530 \\ & & & \end{array}$




$\begin{array}{lrrr}\text { C } & -1.9033100 & -0.4727890 & -0.5558740 \\ \text { C } & -3.9972540 & 1.0400460 & 0.5036180 \\ \text { H } & -2.5612530 & 2.5441830 & 0.8824360 \\ \text { C } & -3.1685340 & -0.9966540 & -0.5431430 \\ \text { H } & -1.1001900 & -1.0586560 & -0.9801250 \\ \text { C } & -4.2711680 & -0.2655020 & -0.0021740 \\ \text { H } & -4.7950120 & 1.6453830 & 0.9152040 \\ \text { H } & -3.3208260 & -1.9892900 & -0.9476570 \\ \text { N } & -5.5196440 & -0.7861620 & 0.0215060 \\ \text { C } & -5.8376310 & -2.0716770 & -0.5981680 \\ \text { C } & -5.5835630 & -3.2469720 & 0.3430940 \\ \text { H } & -5.2707600 & -2.1740500 & -1.5260420 \\ \text { H } & -6.8932280 & -2.0340070 & -0.8824940 \\ \text { H } & -5.8503770 & -4.1868210 & -0.1471040 \\ \text { H } & -4.5310160 & -3.2921680 & 0.6356160 \\ \text { H } & -6.1851830 & -3.1490850 & 1.2513640 \\ \text { C } & -6.6343010 & -0.0965380 & 0.6683460 \\ \text { C } & -7.3251710 & 0.8918840 & -0.2685220 \\ \text { H } & -6.2748530 & 0.3963270 & 1.5743230 \\ \text { H } & -7.3388480 & -0.8673450 & 0.9935490 \\ \text { H } & -8.1615670 & 1.3753450 & 0.2427760 \\ \text { H } & -6.6274030 & 1.6638590 & -0.6042650 \\ \text { H } & -7.7130130 & 0.3767090 & -1.1520820\end{array}$

2 (excited-state/keto) G= -1148.722324 au

$\begin{array}{lrrr}\text { C } & 4.7755290 & -1.2196870 & 0.0443810 \\ \text { C } & 5.4753090 & -0.0057740 & -0.0119030 \\ \text { C } & 6.8502180 & 0.0697200 & -0.0283330 \\ \text { C } & 7.5387870 & -1.1525670 & 0.0158170 \\ \text { C } & 6.8552000 & -2.3670150 & 0.0730750 \\ \text { C } & 5.4578380 & -2.4309710 & 0.0886500 \\ \text { C } & 3.3227800 & 0.4823840 & -0.0119550 \\ \text { H } & 4.9302640 & -3.3764590 & 0.1333260 \\ \text { N } & 3.4449880 & -0.8733190 & 0.0426800 \\ \text { H } & 7.3621290 & 1.0236310 & -0.0726280 \\ \text { O } & 4.5759010 & 1.0294220 & -0.0453420 \\ \text { H } & 7.4211300 & -3.2922570 & 0.1066820 \\ \text { C } & 2.1578480 & 1.2709610 & -0.0291770 \\ \text { C } & 0.8515150 & 0.5797250 & -0.0011430 \\ \text { C } & 2.2247750 & 2.6603110 & -0.0533010 \\ \text { C } & -0.3336840 & 1.4089730 & -0.0273570 \\ \text { C } & 1.0448820 & 3.4371160 & -0.0817810 \\ \text { H } & 3.1949240 & 3.1478410 & -0.0547240 \\ \text { C } & -0.1911970 & 2.8357510 & -0.0585180 \\ \text { O } & 0.8260740 & -0.6781080 & 0.0752090 \\ \text { H } & 8.6231960 & -1.1482690 & 0.0052700 \\ \text { H } & -1.0784450 & 3.4529250 & -0.1413210 \\ \text { H } & 1.1253040 & 4.5178950 & -0.1373590 \\ \text { C } & -1.6547360 & 0.8237230 & -0.0129170 \\ \text { C } & -2.7786850 & 1.5697460 & 0.4310550 \\ \text { C } & -1.9315270 & -0.4983140 & -0.4541890 \\ \text { C } & -4.0550030 & 1.0554660 & 0.4488860 \\ \text { H } & -2.6333590 & 2.5739340 & 0.8142550 \\ \text { C } & -3.2047490 & -1.0197210 & -0.4557050 \\ \text { H } & -1.1117520 & -1.1086160 & -0.8058300 \\ \text { C } & -4.3193620 & -0.2653680 & -0.0000740 \\ \text { H } & -4.8613980 & 1.6840500 & 0.8070010 \\ \text { H } & -3.3427430 & -2.0353180 & -0.8068920 \\ \text { N } & -5.5820230 & -0.7839900 & 0.0042750 \\ \text { C } & -5.8767440 & -2.0931780 & -0.5638220 \\ \text { C } & -5.6591650 & -3.2297890 & 0.4335040\end{array}$




$\begin{array}{lrrr}\text { H } & -5.2755990 & -2.2401600 & -1.4651590 \\ \text { H } & -6.9209400 & -2.0753430 & -0.8918750 \\ \text { H } & -5.8978480 & -4.1937900 & -0.0246030 \\ \text { H } & -4.6205610 & -3.2531780 & 0.7748680 \\ \text { H } & -6.3003610 & -3.0968500 & 1.3101360 \\ \text { C } & -6.7081410 & -0.0581230 & 0.5768350 \\ \text { C } & -7.3558550 & 0.9036660 & -0.4179120 \\ \text { H } & -6.3790570 & 0.4686730 & 1.4767880 \\ \text { H } & -7.4381750 & -0.8033690 & 0.9084380 \\ \text { H } & -8.2007940 & 1.4225200 & 0.0436070 \\ \text { H } & -6.6344870 & 1.6496240 & -0.7630040 \\ \text { H } & -7.7227560 & 0.3584420 & -1.2927400 \\ \text { H } & 2.5897600 & -1.4274990 & 0.0834490\end{array}$

2 (excited-state/ESIPT TS) G= $-1148.716195 \mathrm{au}, v(\mathrm{imag})=1161 i \mathrm{~cm}^{-1}$

\begin{tabular}{|c|c|c|c|}
\hline & 4.5800900 & -1.2099870 & 0.0433480 \\
\hline & 5.4130280 & -0.0751050 & 0.0058870 \\
\hline 0 & 6.7881540 & -0.1393740 & 0.0123340 \\
\hline$C$ & 7.3498940 & -1.4249740 & 0.0596980 \\
\hline$C$ & 6.5427070 & -2.5633680 & 0.0980540 \\
\hline C & 5.1477220 & -2.4833810 & 0.0908530 \\
\hline C & 3.3355460 & 0.5889540 & -0.0243330 \\
\hline$H$ & 4.5261020 & -3.3708940 & 0.1209150 \\
\hline $\mathrm{N}$ & 3.2853790 & -0.7583060 & 0.0237550 \\
\hline $\mathrm{H}$ & 7.3960290 & 0.7574950 & -0.0174750 \\
\hline O & 4.6245380 & 1.0497190 & -0.0360320 \\
\hline$H$ & 7.0115490 & -3.5417920 & 0.1345080 \\
\hline$C$ & 2.2001270 & 1.4141410 & -0.0481110 \\
\hline C & 0.9052970 & 0.7231460 & -0.0312860 \\
\hline C & 2.2328830 & 2.8112300 & -0.0670860 \\
\hline C & -0.2950330 & 1.4945680 & -0.0501510 \\
\hline C & 1.0370880 & 3.5517580 & -0.0897950 \\
\hline $\mathrm{H}$ & 3.1925510 & 3.3180940 & -0.0660190 \\
\hline C & -0.1925350 & 2.9305170 & -0.0649090 \\
\hline $\mathrm{O}$ & 0.8917490 & -0.5719500 & 0.0422270 \\
\hline $\mathrm{H}$ & 2.0555920 & -0.9727000 & 0.0473950 \\
\hline $\mathrm{H}$ & 8.4292650 & -1.5313160 & 0.0666120 \\
\hline H & -1.0929320 & 3.5251370 & -0.1568180 \\
\hline $\mathrm{H}$ & 1.0916520 & 4.6349690 & -0.1405690 \\
\hline C & -1.5960040 & 0.8705700 & -0.0332860 \\
\hline C & -2.7313170 & 1.5843790 & 0.4436030 \\
\hline C & -1.8477810 & -0.4487770 & -0.5056680 \\
\hline C & -3.9910420 & 1.0391220 & 0.4743700 \\
\hline $\mathrm{H}$ & -2.5979150 & 2.5831760 & 0.8432580 \\
\hline C & -3.1043340 & -1.0003390 & -0.4976910 \\
\hline $\mathrm{H}$ & -1.0235770 & -1.0298390 & -0.8943390 \\
\hline C & -4.2301000 & -0.2816320 & -0.0000620 \\
\hline $\mathrm{H}$ & -4.8068260 & 1.6384070 & 0.8592560 \\
\hline $\mathrm{H}$ & -3.2261690 & -2.0085500 & -0.8738250 \\
\hline $\mathrm{N}$ & -5.4725410 & -0.8298590 & 0.0164920 \\
\hline C & -5.7478540 & -2.1378720 & -0.5707490 \\
\hline C & -5.4811160 & -3.2828640 & 0.4041550 \\
\hline $\mathrm{H}$ & -5.1630480 & -2.2527400 & -1.4866470 \\
\hline $\mathrm{H}$ & -6.7989640 & -2.1370530 & -0.8746060 \\
\hline $\mathrm{H}$ & -5.7108800 & -4.2432540 & -0.0651270 \\
\hline $\mathrm{H}$ & -4.4340820 & -3.2900980 & 0.7192490 \\
\hline H & -6.1035450 & -3.1783400 & 1.2977290 \\
\hline C & -6.6107630 & -0.1432500 & 0.6193960 \\
\hline C & -7.2975510 & 0.8123790 & -0.3541930 \\
\hline $\mathrm{H}$ & -6.2809960 & 0.3782440 & 1.5213660 \\
\hline
\end{tabular}




$\begin{array}{rrrr}H & -7.3124380 & -0.9152530 & 0.9492360 \\ H & -8.1519530 & 1.2966630 & 0.1262750 \\ H & -6.6038380 & 1.5858850 & -0.6950180 \\ H & -7.6583790 & 0.2697250 & -1.2329140\end{array}$


3 (ground-state) G= $-1148.846094 \mathrm{au}$

\begin{tabular}{|c|c|c|c|}
\hline C & -5.2682550 & -0.4105310 & 0.0533330 \\
\hline C & -4.3828370 & -1.4879750 & 0.1109840 \\
\hline C & -4.7806740 & -2.8108780 & 0.1950450 \\
\hline C & -6.1570820 & -3.0223780 & 0.2202780 \\
\hline C & -7.0688080 & -1.9553800 & 0.1635920 \\
\hline C & -6.6439860 & -0.6341570 & 0.0793310 \\
\hline C & -3.2786600 & 0.3654020 & -0.0107530 \\
\hline $\mathrm{H}$ & -7.3443450 & 0.1923910 & 0.0355800 \\
\hline $\mathrm{N}$ & -4.5185510 & 0.7597220 & -0.0242460 \\
\hline $\mathrm{H}$ & -4.0653550 & -3.6236820 & 0.2384710 \\
\hline 0 & -3.1118800 & -0.9815390 & 0.0689390 \\
\hline $\mathrm{H}$ & -8.1319150 & -2.1708830 & 0.1864810 \\
\hline C & -2.1011320 & 1.2122550 & -0.0734790 \\
\hline C & -2.2613100 & 2.6115690 & -0.1523520 \\
\hline C & -0.8153730 & 0.6548620 & -0.0414860 \\
\hline C & -1.1154440 & 3.4130530 & -0.2033480 \\
\hline C & 0.3254490 & 1.4481630 & -0.0960870 \\
\hline $\mathrm{H}$ & -0.7219330 & -0.4230980 & 0.0497270 \\
\hline C & 0.1438430 & 2.8415140 & -0.1794120 \\
\hline 0 & -3.4576810 & 3.2219870 & -0.1830690 \\
\hline $\mathrm{H}$ & -4.1698390 & 2.5434570 & -0.1386880 \\
\hline $\mathrm{H}$ & -6.5335380 & -4.0377410 & 0.2854790 \\
\hline C & 1.6797520 & 0.8512000 & -0.0668040 \\
\hline C & 2.7414020 & 1.4769170 & 0.5952560 \\
\hline C & 1.9587100 & -0.3661540 & -0.6970990 \\
\hline C & 4.0138690 & 0.9267470 & 0.6291410 \\
\hline $\mathrm{H}$ & 2.5686140 & 2.4154940 & 1.1158880 \\
\hline C & 3.2218790 & -0.9374170 & -0.6676470 \\
\hline $\mathrm{H}$ & 1.1698980 & -0.8784530 & -1.2422460 \\
\hline C & 4.2943880 & -0.3047530 & -0.0019510 \\
\hline $\mathrm{H}$ & 4.7956860 & 1.4669240 & 1.1497520 \\
\hline $\mathrm{H}$ & 3.3698520 & -1.8880710 & -1.1661100 \\
\hline $\mathrm{H}$ & 1.0163350 & 3.4851910 & -0.2481760 \\
\hline $\mathrm{N}$ & 5.5551840 & -0.8623120 & 0.0296570 \\
\hline C & 6.6223220 & -0.2833740 & 0.8274300 \\
\hline C & 7.3870350 & 0.8190310 & 0.0952240 \\
\hline $\mathrm{H}$ & 7.3076210 & -1.0943050 & 1.0972870 \\
\hline $\mathrm{H}$ & 6.2106300 & 0.0890150 & 1.7710710 \\
\hline $\mathrm{H}$ & 8.1834860 & 1.2263920 & 0.7252620 \\
\hline $\mathrm{H}$ & 7.8400150 & 0.4235510 & -0.8192300 \\
\hline $\mathrm{H}$ & 6.7163100 & 1.6349420 & -0.1889920 \\
\hline C & 5.8775660 & -2.0561300 & -0.7326950 \\
\hline C & 5.5459250 & -3.3496300 & 0.0111200 \\
\hline $\mathrm{H}$ & 6.9488960 & -2.0220750 & -0.9590480 \\
\hline $\mathrm{H}$ & 5.3648370 & -2.0203810 & -1.6993230 \\
\hline $\mathrm{H}$ & 5.8040600 & -4.2246440 & -0.5929670 \\
\hline H & 6.1063810 & -3.4022180 & 0.9497300 \\
\hline $\mathrm{H}$ & 4.4804860 & -3.3963070 & 0.2541270 \\
\hline $\mathrm{H}$ & -1.2476030 & 4.4873000 & -0.2746300 \\
\hline
\end{tabular}

3 (ground-state/keto) G= -1148.824466 au

$\begin{array}{lrrr}\text { C } & -5.3369330 & -0.3371480 & 0.0495010 \\ \text { C } & -4.5011700 & -1.4511490 & 0.1096820 \\ \text { C } & -4.9688310 & -2.7461380 & 0.1944210 \\ \text { C } & -6.3574740 & -2.8889790 & 0.2176730 \\ \text { C } & -7.2091270 & -1.7796750 & 0.1582890 \\ \text { C } & -6.7177590 & -0.4775400 & 0.0727440 \\ \text { C } & -3.2164480 & 0.3188270 & -0.0130820 \\ \text { H } & -7.3763320 & 0.3817750 & 0.0268820\end{array}$




\begin{tabular}{|c|c|c|c|}
\hline $\mathrm{N}$ & -4.4815030 & 0.7538940 & -0.0273180 \\
\hline $\mathrm{H}$ & -4.2942700 & -3.5926500 & 0.2397530 \\
\hline $\mathrm{O}$ & -3.1903120 & -1.0245060 & 0.0693670 \\
\hline $\mathrm{H}$ & -8.2822820 & -1.9355110 & 0.1793440 \\
\hline$C$ & -2.0750940 & 1.1277050 & -0.0754100 \\
\hline$C$ & -2.3011660 & 2.5608060 & -0.1564280 \\
\hline C & -0.7705170 & 0.5669720 & -0.0421600 \\
\hline$C$ & -1.0951780 & 3.3524070 & -0.2085050 \\
\hline C & 0.3465020 & 1.3623440 & -0.0964420 \\
\hline $\mathrm{H}$ & -0.6713740 & -0.5116880 & 0.0508380 \\
\hline C & 0.1421560 & 2.7790180 & -0.1813620 \\
\hline O & -3.4501990 & 3.0814800 & -0.1825790 \\
\hline $\mathrm{H}$ & -6.7837350 & -3.8838950 & 0.2833610 \\
\hline C & 1.7142130 & 0.7988350 & -0.0696050 \\
\hline C & 2.7659180 & 1.4534960 & 0.5813100 \\
\hline C & 2.0205920 & -0.4190020 & -0.6872770 \\
\hline C & 4.0503710 & 0.9305420 & 0.6186820 \\
\hline $\mathrm{H}$ & 2.5769340 & 2.3952930 & 1.0904330 \\
\hline C & 3.2950360 & -0.9652030 & -0.6507820 \\
\hline $\mathrm{H}$ & 1.2421100 & -0.9518510 & -1.2276130 \\
\hline C & 4.3553690 & -0.3026680 & 0.0041870 \\
\hline $\mathrm{H}$ & 4.8220340 & 1.4948360 & 1.1289880 \\
\hline $\mathrm{H}$ & 3.4620140 & -1.9183330 & -1.1387900 \\
\hline $\mathrm{H}$ & 1.0193170 & 3.4186700 & -0.2488600 \\
\hline $\mathrm{N}$ & 5.6291960 & -0.8342430 & 0.0397260 \\
\hline C & 6.6872700 & -0.2181970 & 0.8204630 \\
\hline C & 7.4208070 & 0.8913100 & 0.0669200 \\
\hline $\mathrm{H}$ & 7.3938660 & -1.0081550 & 1.0979590 \\
\hline $\mathrm{H}$ & 6.2731970 & 0.1588890 & 1.7612680 \\
\hline $\mathrm{H}$ & 8.2101060 & 1.3285230 & 0.6859610 \\
\hline $\mathrm{H}$ & 7.8789030 & 0.4928410 & -0.8437450 \\
\hline H & 6.7281930 & 1.6854250 & -0.2263460 \\
\hline C & 5.9756170 & -2.0261980 & -0.7138030 \\
\hline C & 5.6756710 & -3.3208180 & 0.0417490 \\
\hline $\mathrm{H}$ & 7.0451910 & -1.9708500 & -0.9446440 \\
\hline H & 5.4586830 & -2.0107100 & -1.6788880 \\
\hline $\mathrm{H}$ & 5.9505920 & -4.1952370 & -0.5559150 \\
\hline H & 6.2406920 & -3.3528590 & 0.9785760 \\
\hline H & 4.6124340 & -3.3884410 & 0.2894770 \\
\hline $\mathrm{H}$ & -1.2195360 & 4.4278370 & -0.2834660 \\
\hline $\mathrm{H}$ & -4.5954550 & 1.7854580 & -0.0903110 \\
\hline
\end{tabular}

3 (ground-state/PT TS) G= $-1148.825978 \mathrm{au}, v(\mathrm{imag})=497 i \mathrm{~cm}^{-1}$

$\begin{array}{lrrr}\text { C } & -5.3419530 & -0.3021040 & 0.0456120 \\ \text { C } & -4.5503250 & -1.4491150 & 0.1153480 \\ \text { C } & -5.0666840 & -2.7258560 & 0.2045890 \\ \text { C } & -6.4590210 & -2.8150060 & 0.2220150 \\ \text { C } & -7.2682900 & -1.6736470 & 0.1528530 \\ \text { C } & -6.7281830 & -0.3923400 & 0.0631600 \\ \text { C } & -3.2149430 & 0.2680010 & -0.0102140 \\ \text { H } & -7.3531640 & 0.4912760 & 0.0097770 \\ \text { N } & -4.4497890 & 0.7590710 & -0.0329490 \\ \text { H } & -4.4252960 & -3.5973790 & 0.2573170 \\ \text { O } & -3.2234580 & -1.0727230 & 0.0787240 \\ \text { H } & -8.3465990 & -1.7897480 & 0.1696280 \\ \text { C } & -2.0582070 & 1.0714710 & -0.0741530 \\ \text { C } & -2.2955640 & 2.4948160 & -0.1595970 \\ \text { C } & -0.7549580 & 0.5233950 & -0.0393340 \\ \text { C } & -1.1116540 & 3.3013470 & -0.2146510 \\ \text { C } & 0.3567710 & 1.3329520 & -0.0969370\end{array}$




$\begin{array}{llll}\text { H } & -0.6454300 & -0.5540480 & 0.0565900 \\ \text { C } & 0.1368010 & 2.7409810 & -0.1864830 \\ \text { O } & -3.4660690 & 3.0044330 & -0.1869700 \\ \text { H } & -4.4212160 & 1.8523440 & -0.1054800 \\ \text { H } & -6.9237140 & -3.7924060 & 0.2908820 \\ \text { C } & 1.7295220 & 0.7811820 & -0.0688080 \\ \text { C } & 2.7748220 & 1.4450030 & 0.5828740 \\ \text { C } & 2.0463830 & -0.4336080 & -0.6868820 \\ \text { C } & 4.0639790 & 0.9338610 & 0.6196200 \\ \text { H } & 2.5766600 & 2.3844950 & 1.0927750 \\ \text { C } & 3.3258200 & -0.9680510 & -0.6511280 \\ \text { H } & 1.2726560 & -0.9736290 & -1.2269770 \\ \text { C } & 4.3801320 & -0.2961320 & 0.0040630 \\ \text { H } & 4.8304900 & 1.5046240 & 1.1304750 \\ \text { H } & 3.5012950 & -1.9195030 & -1.1393630 \\ \text { H } & 1.0049900 & 3.3922080 & -0.2567860 \\ \text { N } & 5.6585760 & -0.8160200 & 0.0391680 \\ \text { C } & 6.7107640 & -0.1918190 & 0.8215240 \\ \text { C } & 7.4353940 & 0.9246220 & 0.0696680 \\ \text { H } & 7.4237890 & -0.9760700 & 1.0987300 \\ \text { H } & 6.2927750 & 0.1807270 & 1.7624040 \\ \text { H } & 8.2203480 & 1.3679710 & 0.6898510 \\ \text { H } & 7.8976940 & 0.5309660 & -0.8409580 \\ \text { H } & 6.7362580 & 1.7130620 & -0.2234460 \\ \text { C } & 6.0153290 & -2.0055690 & -0.7134650 \\ \text { C } & 5.7264000 & -3.3021450 & 0.0429920 \\ \text { H } & 7.0844020 & -1.9410970 & -0.9441470 \\ \text { H } & 5.4984710 & -1.9951420 & -1.6786350 \\ \text { H } & 6.0091760 & -4.1745540 & -0.5539230 \\ \text { H } & 6.2913570 & -3.3285520 & 0.9800300 \\ \text { H } & 4.6637060 & -3.3789270 & 0.2903800 \\ \text { H } & -1.2479370 & 4.3749060 & -0.2926820\end{array}$

3 (excited-state/enol) G= $-1148.719135 \mathrm{au}$

$\begin{array}{llll}\text { C } & -5.2876840 & -0.3384470 & 0.0103900 \\ \text { C } & -4.4470280 & -1.4701670 & 0.0197000 \\ \text { C } & -4.9045550 & -2.7693800 & 0.0365210 \\ \text { C } & -6.2981300 & -2.9365000 & 0.0444450 \\ \text { C } & -7.1555640 & -1.8319080 & 0.0354940 \\ \text { C } & -6.6753030 & -0.5220730 & 0.0184140 \\ \text { C } & -3.2436590 & 0.3448710 & -0.0067080 \\ \text { H } & -7.3457690 & 0.3302140 & 0.0117130 \\ \text { N } & -4.5084320 & 0.7873740 & -0.0055640 \\ \text { H } & -4.2214980 & -3.6113200 & 0.0433410 \\ \text { O } & -3.1497600 & -1.0332650 & 0.0083400 \\ \text { H } & -8.2285970 & -1.9985910 & 0.0421300 \\ \text { C } & -2.0757740 & 1.1302650 & -0.0258370 \\ \text { C } & -2.2046820 & 2.5781780 & -0.0367350 \\ \text { C } & -0.7750930 & 0.5968810 & -0.0188000 \\ \text { C } & -1.0717910 & 3.3911620 & -0.0362210 \\ \text { C } & 0.3677320 & 1.4277510 & -0.0094380 \\ \text { H } & -0.6715010 & -0.4789890 & 0.0518160 \\ \text { C } & 0.2095470 & 2.8362080 & -0.0098340 \\ \text { O } & -3.4015610 & 3.1482960 & -0.0462490 \\ \text { H } & -4.1077230 & 2.4293150 & -0.0372600 \\ \text { H } & -6.7128530 & -3.9388410 & 0.0576260 \\ \text { C } & 1.6901600 & 0.8420400 & -0.0054240 \\ \text { C } & 2.8312140 & 1.5740080 & 0.4139610 \\ \text { C } & 1.9190820 & -0.4955930 & -0.4200750 \\ \text { C } & 4.0895100 & 1.0278810 & 0.4278320\end{array}$




$\begin{array}{lrrr}\text { H } & 2.7076300 & 2.5902850 & 0.7698480 \\ \mathrm{C} & 3.1708170 & -1.0577110 & -0.4257420 \\ \mathrm{H} & 1.0866570 & -1.0906260 & -0.7769840 \\ \mathrm{C} & 4.3110570 & -0.3160060 & 0.0023420 \\ \mathrm{H} & 4.9189230 & 1.6401420 & 0.7580190 \\ \mathrm{H} & 3.2779600 & -2.0844580 & -0.7512860 \\ \mathrm{H} & 1.0711280 & 3.4887640 & -0.0766940 \\ \mathrm{~N} & 5.5489350 & -0.8625510 & 0.0047510 \\ \mathrm{C} & 6.7088000 & -0.1532260 & 0.5416820 \\ \mathrm{C} & 7.3679370 & 0.7506170 & -0.4973630 \\ \mathrm{H} & 7.4154540 & -0.9140800 & 0.8854490 \\ \mathrm{H} & 6.4080300 & 0.4104510 & 1.4276880 \\ \mathrm{H} & 8.2391590 & 1.2505430 & -0.0662300 \\ \mathrm{H} & 7.6987450 & 0.1655980 & -1.3604040 \\ \mathrm{H} & 6.6684200 & 1.5124920 & -0.8521560 \\ \mathrm{C} & 5.8077890 & -2.1978580 & -0.5308950 \\ \mathrm{C} & 5.5851870 & -3.2928530 & 0.5093850 \\ \mathrm{H} & 6.8460400 & -2.2064790 & -0.8748590 \\ \mathrm{H} & 5.1885420 & -2.3569320 & -1.4165230 \\ \mathrm{H} & 5.8038070 & -4.2737740 & 0.0795770 \\ \mathrm{H} & 6.2401580 & -3.1411890 & 1.3723390 \\ \mathrm{H} & 4.5508860 & -3.2899820 & 0.8641080 \\ \mathrm{H} & -1.2239620 & 4.4646020 & -0.0648580\end{array}$

3 (excited-state/keto) G= $-1148.721592 \mathrm{au}$

$\begin{array}{lccc}\text { C } & -5.3259020 & -0.3566330 & 0.0138150 \\ \text { C } & -4.4538690 & -1.4566400 & 0.0219070 \\ \text { C } & -4.8900970 & -2.7630650 & 0.0396540 \\ \text { C } & -6.2811100 & -2.9510000 & 0.0495570 \\ \text { C } & -7.1593280 & -1.8663180 & 0.0415880 \\ \text { C } & -6.7057570 & -0.5439820 & 0.0235100 \\ \text { C } & -3.2103800 & 0.3625920 & -0.0058600 \\ \text { H } & -7.3905960 & 0.2957780 & 0.0175100 \\ \text { N } & -4.5142230 & 0.7503050 & -0.0028760 \\ \text { H } & -4.1916550 & -3.5913740 & 0.0457090 \\ \text { O } & -3.1594300 & -1.0023580 & 0.0091090 \\ \text { H } & -8.2285240 & -2.0516490 & 0.0497740 \\ \text { C } & -2.0593070 & 1.1762510 & -0.0300500 \\ \text { C } & -2.2511620 & 2.6427920 & -0.0432320 \\ \text { C } & -0.7790260 & 0.6359800 & -0.0232690 \\ \text { C } & -1.0582030 & 3.4267630 & -0.0422130 \\ \text { C } & 0.3858660 & 1.4612090 & -0.0170390 \\ \text { H } & -0.6744050 & -0.4420370 & 0.0372680 \\ \text { C } & 0.2083120 & 2.8623470 & -0.0228600 \\ \text { O } & -3.4066310 & 3.1425710 & -0.0528550 \\ \text { H } & -6.6774080 & -3.9603780 & 0.0636740 \\ \text { C } & 1.7045320 & 0.8614370 & -0.0056740 \\ \text { C } & 2.8485510 & 1.5758860 & 0.4203650 \\ \text { C } & 1.9268070 & -0.4721540 & -0.4205880 \\ \text { C } & 4.1065810 & 1.0184130 & 0.4300640 \\ \text { H } & 2.7371030 & 2.5925870 & 0.7818340 \\ \text { C } & 3.1798620 & -1.0460850 & -0.4236190 \\ \text { H } & 1.0943210 & -1.0653800 & -0.7834300 \\ \text { C } & 4.3214510 & -0.3201900 & 0.0031240 \\ \text { H } & 4.9383600 & 1.6251710 & 0.7662470 \\ \text { H } & 3.2765390 & -2.0737680 & -0.7516000 \\ \text { H } & 1.0743810 & 3.5139020 & -0.0701560 \\ \text { N } & 5.5663600 & -0.8792860 & 0.0067170 \\ \text { C } & 6.7221350 & -0.1784670 & 0.5519870 \\ \text { C } & 7.3891680 & 0.7409500 & -0.4693290\end{array}$




$\begin{array}{rrrr}\text { H } & 7.4311890 & -0.9402680 & 0.8911040 \\ \text { H } & 6.4214160 & 0.3764470 & 1.4449390 \\ \text { H } & 8.2564200 & 1.2403490 & -0.0283140 \\ \text { H } & 7.7270530 & 0.1662190 & -1.3368970 \\ \text { H } & 6.6895840 & 1.5039700 & -0.8219870 \\ \text { C } & 5.8116900 & -2.2078390 & -0.5395900 \\ \text { C } & 5.5687140 & -3.3182980 & 0.4808170 \\ \text { H } & 6.8519950 & -2.2305980 & -0.8791810 \\ \text { H } & 5.1964840 & -2.3512230 & -1.4322230 \\ \text { H } & 5.7700180 & -4.2979170 & 0.0383130 \\ \text { H } & 6.2244360 & -3.1906330 & 1.3473870 \\ \text { H } & 4.5343040 & -3.3017760 & 0.8353310 \\ \text { H } & -1.1899010 & 4.5035400 & -0.0723750 \\ \text { H } & -4.7047570 & 1.7564980 & -0.0182320\end{array}$

3 (excited-state/ESIPT TS) G= $-1148.717508 \mathrm{au}, v(\mathrm{imag})=1159 i \mathrm{~cm}^{-1}$

$\begin{array}{lccr}\text { C } & -5.3346380 & -0.2618060 & 0.0087960 \\ \text { C } & -4.5768690 & -1.4507350 & 0.0269330 \\ \text { C } & -5.1345540 & -2.7091510 & 0.0506140 \\ \text { C } & -6.5374100 & -2.7669340 & 0.0557610 \\ \text { C } & -7.3072290 & -1.6017000 & 0.0373850 \\ \text { C } & -6.7296270 & -0.3303280 & 0.0136290 \\ \text { C } & -3.2042980 & 0.2435450 & -0.0053510 \\ \text { H } & -7.3328540 & 0.5700610 & -0.0001370 \\ \text { N } & -4.4449720 & 0.7779280 & -0.0105020 \\ \text { H } & -4.5178350 & -3.6003410 & 0.0645180 \\ \text { O } & -3.2420660 & -1.1248910 & 0.0170010 \\ \text { H } & -8.3896160 & -1.6850490 & 0.0419270 \\ \text { C } & -2.0312460 & 1.0164830 & -0.0298220 \\ \text { C } & -2.2285740 & 2.4747050 & -0.0450610 \\ \text { C } & -0.7344060 & 0.5051420 & -0.0224370 \\ \text { C } & -1.0859140 & 3.2999760 & -0.0446160 \\ \text { C } & 0.3994410 & 1.3629760 & -0.0143210 \\ \text { H } & -0.6069220 & -0.5693100 & 0.0451430 \\ \text { C } & 0.1977970 & 2.7640010 & -0.0197710 \\ \text { O } & -3.4238460 & 2.9643250 & -0.0557500 \\ \text { H } & -4.2271910 & 1.9951700 & -0.0365370 \\ \text { H } & -7.0277170 & -3.7342050 & 0.0742150 \\ \text { C } & 1.7336710 & 0.8035590 & -0.0060410 \\ \text { C } & 2.8582280 & 1.5521670 & 0.4200920 \\ \text { C } & 1.9909100 & -0.5253980 & -0.4233160 \\ \text { C } & 4.1287640 & 1.0296730 & 0.4326910 \\ \text { H } & 2.7169990 & 2.5647850 & 0.7814450 \\ \text { C } & 3.2564490 & -1.0635170 & -0.4286120 \\ \text { H } & 1.1730510 & -1.1381970 & -0.7853970 \\ \text { C } & 4.3790940 & -0.3046170 & 0.0024090 \\ \text { H } & 4.9443210 & 1.6571500 & 0.7695570 \\ \text { H } & 3.3828230 & -2.0869960 & -0.7584670 \\ \text { H } & 1.0480130 & 3.4338770 & -0.0752460 \\ \text { N } & 5.6330920 & -0.8279630 & 0.0053840 \\ \text { C } & 6.7726690 & -0.0993530 & 0.5539990 \\ \text { C } & 7.4184190 & 0.8331920 & -0.4684550 \\ \text { H } & 7.4966900 & -0.8461460 & 0.8937040 \\ \text { H } & 6.4563200 & 0.4478550 & 1.4456330 \\ \text { H } & 8.2750750 & 1.3492660 & -0.0265980 \\ \text { H } & 7.7682080 & 0.2663300 & -1.3363020 \\ \text { H } & 6.7033610 & 1.5822820 & -0.8197570 \\ & 5.9173170 & -2.1511930 & -0.5410220 \\ & 6.9567640 & -2.1403770 & -0.8825520\end{array}$




$\begin{array}{rrrr}H & 5.3042160 & -2.3133350 & -1.4311310 \\ H & 5.9436990 & -4.2370860 & 0.0435880 \\ H & 6.3628360 & -3.1129340 & 1.3496150 \\ \text { H } & 4.6765040 & -3.2802980 & 0.8388380 \\ \text { H } & -1.2489840 & 4.3717770 & -0.0761230\end{array}$


4 (ground-state) G= $-1245.902288 \mathrm{au}$

\begin{tabular}{|c|c|c|c|}
\hline C & -4.4019540 & 1.1464640 & -0.0105480 \\
\hline C & -4.5069120 & -0.2449920 & 0.0096400 \\
\hline C & -5.7100770 & -0.9288300 & 0.0203900 \\
\hline C & -6.8519420 & -0.1316690 & 0.0091830 \\
\hline C & -6.7731400 & 1.2707380 & -0.0115590 \\
\hline C & -5.5516870 & 1.9346480 & -0.0217720 \\
\hline C & -2.4251650 & 0.3340670 & 0.0012710 \\
\hline $\mathrm{H}$ & -5.4863490 & 3.0167680 & -0.0377300 \\
\hline$N$ & -3.0492730 & 1.4752940 & -0.0151900 \\
\hline $\mathrm{H}$ & -5.7549810 & -2.0113680 & 0.0366800 \\
\hline 0 & -3.2368590 & -0.7558670 & 0.0163970 \\
\hline $\mathrm{H}$ & -7.6913890 & 1.8484970 & -0.0197580 \\
\hline C & -0.9863940 & 0.1300690 & 0.0022980 \\
\hline C & -0.1304580 & 1.2525630 & -0.0025150 \\
\hline C & -0.4595560 & -1.1668760 & 0.0118530 \\
\hline C & 1.2666080 & 1.0510980 & -0.0011670 \\
\hline C & 0.9125190 & -1.3809140 & 0.0122200 \\
\hline $\mathrm{H}$ & -1.1450040 & -2.0079510 & 0.0452570 \\
\hline C & 1.7488990 & -0.2541950 & 0.0054620 \\
\hline 0 & -0.5929180 & 2.5120860 & -0.0242180 \\
\hline $\mathrm{H}$ & -1.5793010 & 2.4990040 & -0.0389190 \\
\hline $\mathrm{H}$ & -7.8270280 & -0.6071030 & 0.0167620 \\
\hline C & 1.4761430 & -2.7523790 & 0.0307050 \\
\hline C & 2.6275420 & -3.0489690 & 0.7686620 \\
\hline C & 0.8667450 & -3.7905020 & -0.6833910 \\
\hline C & 3.1490030 & -4.3378240 & 0.7891240 \\
\hline $\mathrm{H}$ & 3.1037150 & -2.2685520 & 1.3560230 \\
\hline C & 1.3898140 & -5.0781060 & -0.6560550 \\
\hline $\mathrm{H}$ & -0.0159200 & -3.5820850 & -1.2820600 \\
\hline C & 2.5417900 & -5.3746610 & 0.0768350 \\
\hline $\mathrm{H}$ & 4.0381770 & -4.5456830 & 1.3792500 \\
\hline $\mathrm{H}$ & 0.8994630 & -5.8670660 & -1.2211490 \\
\hline C & 2.2161270 & 2.1930680 & -0.0356410 \\
\hline C & 2.0514510 & 3.3155530 & 0.7859910 \\
\hline C & 3.3368070 & 2.1447960 & -0.8705350 \\
\hline C & 2.9828670 & 4.3454760 & 0.7712570 \\
\hline $\mathrm{H}$ & 1.1910190 & 3.3737340 & 1.4433730 \\
\hline C & 4.2647360 & 3.1818310 & -0.8826910 \\
\hline $\mathrm{H}$ & 3.4752340 & 1.2917320 & -1.5295060 \\
\hline C & 4.1035600 & 4.3000550 & -0.0641340 \\
\hline $\mathrm{H}$ & 2.8406560 & 5.2033910 & 1.4241930 \\
\hline $\mathrm{H}$ & 5.1254790 & 3.1228040 & -1.5441730 \\
\hline $\mathrm{H}$ & 2.8262290 & -0.3995760 & 0.0018580 \\
\hline C & 3.1249340 & -6.7646270 & 0.0771150 \\
\hline $\mathrm{H}$ & 2.3432390 & -7.5224090 & -0.0246700 \\
\hline $\mathrm{H}$ & 3.8218600 & -6.8966680 & -0.7581430 \\
\hline $\mathrm{H}$ & 3.6763880 & -6.9625670 & 1.0001720 \\
\hline C & 5.0896060 & 5.4397300 & -0.0935860 \\
\hline $\mathrm{H}$ & 4.7042500 & 6.2749350 & -0.6889590 \\
\hline $\mathrm{H}$ & 5.2851200 & 5.8198490 & 0.9133790 \\
\hline $\mathrm{H}$ & 6.0410720 & 5.1298480 & -0.5334640 \\
\hline
\end{tabular}

4 (ground-state/keto) G= $-1245.881960 \mathrm{au}$

$\begin{array}{llll}\text { C } & 4.4976560 & -0.7652000 & -0.0016390 \\ \text { C } & 4.4995990 & 0.6280660 & -0.0021010 \\ \text { C } & 5.6530570 & 1.3849740 & -0.0026060 \\ \text { C } & 6.8489800 & 0.6652070 & -0.0028960 \\ \text { C } & 6.8624140 & -0.7348180 & -0.0027320 \\ \text { C } & 5.6859180 & -1.4827260 & -0.0020280\end{array}$




\begin{tabular}{|c|c|c|c|}
\hline C & 2.4057750 & -0.0178370 & -0.0001660 \\
\hline$H$ & 5.6949620 & -2.5662590 & -0.0018780 \\
\hline$N$ & 3.1556460 & -1.1246850 & 0.0000070 \\
\hline $\mathrm{H}$ & 5.6231430 & 2.4678950 & -0.0025260 \\
\hline $\mathrm{O}$ & 3.1940730 & 1.0722540 & -0.0019180 \\
\hline H & 7.8146670 & -1.2539160 & -0.0030600 \\
\hline 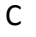 & 1.0040300 & 0.0351680 & -0.0004640 \\
\hline$C$ & 0.3038300 & -1.2382000 & 0.0083710 \\
\hline C & 0.3299680 & 1.2817450 & 0.0034890 \\
\hline C & -1.1516930 & -1.1426460 & 0.0071360 \\
\hline C & -1.0407750 & 1.3330080 & 0.0119130 \\
\hline $\mathrm{H}$ & 0.9177900 & 2.1955500 & 0.0302080 \\
\hline C & -1.7495360 & 0.0930680 & 0.0157220 \\
\hline 0 & 0.9118380 & -2.3414220 & 0.0065280 \\
\hline $\mathrm{H}$ & 7.7890340 & 1.2056620 & -0.0032450 \\
\hline C & -1.7735570 & 2.6200790 & 0.0281090 \\
\hline C & -2.9645970 & 2.7675960 & 0.7494730 \\
\hline C & -1.2966110 & 3.7312930 & -0.6787270 \\
\hline C & -3.6470190 & 3.9791540 & 0.7643550 \\
\hline $\mathrm{H}$ & -3.3519220 & 1.9296360 & 1.3227370 \\
\hline C & -1.9786160 & 4.9420920 & -0.6549060 \\
\hline $\mathrm{H}$ & -0.3945190 & 3.6348730 & -1.2769450 \\
\hline C & -3.1656230 & 5.0895700 & 0.0674760 \\
\hline $\mathrm{H}$ & -4.5686000 & 4.0664300 & 1.3348960 \\
\hline $\mathrm{H}$ & -1.5909800 & 5.7858750 & -1.2209630 \\
\hline C & -1.9754010 & -2.3745260 & -0.0349740 \\
\hline C & -1.6456620 & -3.5101590 & 0.7188710 \\
\hline C & -3.1383610 & -2.4195650 & -0.8119270 \\
\hline C & -2.4627460 & -4.6332690 & 0.7023330 \\
\hline $\mathrm{H}$ & -0.7434410 & -3.5030680 & 1.3186940 \\
\hline C & -3.9510940 & -3.5490980 & -0.8259060 \\
\hline $\mathrm{H}$ & -3.4007380 & -1.5639980 & -1.4286180 \\
\hline C & -3.6279260 & -4.6759990 & -0.0699190 \\
\hline $\mathrm{H}$ & -2.1924690 & -5.4986400 & 1.3034580 \\
\hline $\mathrm{H}$ & -4.8469800 & -3.5572920 & -1.4420980 \\
\hline $\mathrm{H}$ & -2.8369330 & 0.1325990 & 0.0190980 \\
\hline C & -3.8901630 & 6.4108010 & 0.1108160 \\
\hline $\mathrm{H}$ & -3.7592790 & 6.9653180 & -0.8226170 \\
\hline $\mathrm{H}$ & -4.9615280 & 6.2702250 & 0.2778870 \\
\hline $\mathrm{H}$ & -3.5098520 & 7.0405640 & 0.9231040 \\
\hline C & -4.4870640 & -5.9143310 & -0.1019420 \\
\hline $\mathrm{H}$ & -4.0305770 & -6.6932650 & -0.7228840 \\
\hline $\mathrm{H}$ & -4.6167120 & -6.3322530 & 0.9009550 \\
\hline $\mathrm{H}$ & -5.4770110 & -5.6997400 & -0.5130690 \\
\hline $\mathrm{H}$ & 2.6364410 & -2.0207530 & -0.0008470 \\
\hline
\end{tabular}

4 (ground-state/PT TS) G= $-1245.883084 \mathrm{au}, v(\mathrm{imag})=601 \mathrm{icm}-1$

$\begin{array}{lrrr}\text { C } & -4.5033650 & 0.4042550 & -0.0000170 \\ \text { C } & -4.4483290 & -0.9898170 & -0.0034690 \\ \text { C } & -5.5689850 & -1.7959140 & -0.0083390 \\ \text { C } & -6.7931070 & -1.1271300 & -0.0099840 \\ \text { C } & -6.8660850 & 0.2723120 & -0.0068210 \\ \text { C } & -5.7233880 & 1.0689570 & -0.0016710 \\ \text { C } & -2.4019340 & -0.2465130 & 0.0039030 \\ \text { H } & -5.7777350 & 2.1512110 & 0.0006990 \\ \text { N } & -3.1803160 & 0.8274400 & 0.0054020 \\ \text { H } & -5.4939270 & -2.8766620 & -0.0106750 \\ \text { O } & -3.1242660 & -1.3783060 & -0.0014510 \\ \text { H } & -7.8400610 & 0.7495870 & -0.0084040 \\ \text { C } & -0.9891690 & -0.1884570 & 0.0040830\end{array}$




$\begin{array}{lccc}\text { C } & -0.4220680 & 1.1399960 & 0.0123420 \\ \text { C } & -0.1999060 & -1.3570090 & 0.0060390 \\ \text { C } & 1.0226320 & 1.2043580 & 0.0067170 \\ \text { C } & 1.1736110 & -1.2678530 & 0.0126040 \\ \text { H } & -0.6933260 & -2.3251310 & 0.0328360 \\ \text { C } & 1.7481590 & 0.0322660 & 0.0143700 \\ \text { O } & -1.1534790 & 2.1881780 & 0.0132350 \\ \text { H } & -2.5529600 & 1.7360390 & 0.0066040 \\ \text { H } & -7.7097170 & -1.7065830 & -0.0139020 \\ \text { C } & 2.0322900 & -2.4751100 & 0.0291360 \\ \text { C } & 3.2269300 & -2.5037010 & 0.7599320 \\ \text { C } & 1.6705060 & -3.6281650 & -0.6770510 \\ \text { C } & 4.0247790 & -3.6415720 & 0.7814920 \\ \text { H } & 3.5205830 & -1.6336500 & 1.3409820 \\ \text { C } & 2.4688450 & -4.7665790 & -0.6466300 \\ \text { H } & 0.7622490 & -3.6253480 & -1.2737420 \\ \text { C } & 3.6618840 & -4.7935360 & 0.0784460 \\ \text { H } & 4.9422790 & -3.6403870 & 1.3652460 \\ \text { H } & 2.1651540 & -5.6481210 & -1.2061120 \\ \text { C } & 1.7165460 & 2.5146200 & -0.0365100 \\ \text { C } & 1.2665340 & 3.6144100 & 0.7075520 \\ \text { C } & 2.8756910 & 2.6738830 & -0.8046460 \\ \text { C } & 1.9647890 & 4.8151210 & 0.6905670 \\ \text { H } & 0.3655140 & 3.5186410 & 1.3014320 \\ \text { C } & 3.5690860 & 3.8799520 & -0.8185460 \\ \text { H } & 3.2300980 & 1.8466150 & -1.4138530 \\ \text { C } & 3.1260110 & 4.9723870 & -0.0721680 \\ \text { H } & 1.6025430 & 5.6510730 & 1.2847940 \\ \text { H } & 4.4653580 & 3.9757730 & -1.4267260 \\ \text { H } & 2.8334990 & 0.1070080 & 0.0164340 \\ \text { C } & 4.5457610 & -6.0146780 & 0.0831500 \\ \text { H } & 3.9776230 & -6.9161660 & -0.1611850 \\ \text { H } & 5.3498350 & -5.9200050 & -0.6553100 \\ \text { H } & 5.0150700 & -6.1601680 & 1.0603430 \\ \text { C } & 3.8550570 & 6.2913780 & -0.1071450 \\ \text { H } & 3.3613150 & 6.9949370 & -0.7869000 \\ \text { H } & 3.8789640 & 6.7568160 & 0.8826250 \\ \text { H } & 4.8851600 & 6.1662900 & -0.4513140\end{array}$

4 (excited-state/enol) G $=-1245.769744$ au

$\begin{array}{llll}\mathrm{C} & -4.4542280 & -0.7694340 & 0.0035350 \\ \mathrm{C} & -4.4983580 & 0.6397170 & 0.0001750 \\ \mathrm{C} & -5.6659460 & 1.3725710 & 0.0040590 \\ \mathrm{C} & -6.8555980 & 0.6331270 & 0.0124110 \\ \mathrm{C} & -6.8406860 & -0.7678620 & 0.0167100 \\ \mathrm{C} & -5.6521020 & -1.4939240 & 0.0124860 \\ \mathrm{C} & -2.4299440 & -0.0249490 & -0.0090130 \\ \mathrm{H} & -5.6443500 & -2.5780700 & 0.0157990 \\ \mathrm{~N} & -3.1419110 & -1.1592080 & -0.0034090 \\ \mathrm{H} & -5.6568740 & 2.4564120 & 0.0008250 \\ \mathrm{O} & -3.2116070 & 1.1055480 & -0.0067830 \\ \mathrm{H} & -7.7851340 & -1.3030110 & 0.0234170 \\ \mathrm{C} & -1.0251270 & 0.0776100 & -0.0056070 \\ \mathrm{C} & -0.2456600 & -1.1626430 & -0.0136100 \\ \mathrm{C} & -0.3329180 & 1.2923670 & 0.0097770 \\ \mathrm{C} & 1.1751140 & -1.1366260 & -0.0404930 \\ \mathrm{C} & 1.0820400 & 1.3240010 & -0.0216060 \\ \mathrm{H} & -0.9025390 & 2.2134110 & -0.0158370 \\ \mathrm{C} & 1.8136940 & 0.1212220 & -0.0480420 \\ \mathrm{O} & -0.8730570 & -2.3099970 & 0.0454510 \\ \mathrm{H} & -1.8899840 & -2.1490560 & 0.0448870 \\ \mathrm{H} & -7.8051610 & 1.1575200 & 0.0156510\end{array}$




$\begin{array}{lrrr}\text { C } & 1.7789840 & 2.6124910 & -0.0385900 \\ \text { C } & 3.0520560 & 2.7457890 & -0.6239040 \\ \text { C } & 1.1965430 & 3.7604630 & 0.5303000 \\ \text { C } & 3.7047930 & 3.9681230 & -0.6397230 \\ \text { H } & 3.5120840 & 1.8903610 & -1.1076910 \\ \text { C } & 1.8619790 & 4.9766710 & 0.5218270 \\ \text { H } & 0.2278910 & 3.6878130 & 1.0138130 \\ \text { C } & 3.1269050 & 5.1052680 & -0.0623400 \\ \text { H } & 4.6786050 & 4.0491520 & -1.1153020 \\ \text { H } & 1.3954180 & 5.8453820 & 0.9786850 \\ \text { C } & 1.9629110 & -2.3737930 & 0.0059780 \\ \text { C } & 1.5713280 & -3.5550900 & -0.6553040 \\ \text { C } & 3.1897800 & -2.3904470 & 0.6941460 \\ \text { C } & 2.3716980 & -4.6858900 & -0.6205900 \\ \text { H } & 0.6461220 & -3.5727480 & -1.2181020 \\ \text { C } & 3.9793480 & -3.5314020 & 0.7297250 \\ \text { H } & 3.5075060 & -1.5054050 & 1.2373430 \\ \text { C } & 3.5874530 & -4.7015120 & 0.0743880 \\ \text { H } & 2.0530210 & -5.5792740 & -1.1523640 \\ \text { H } & 4.9152200 & -3.5170630 & 1.2825970 \\ \text { H } & 2.8957590 & 0.1525480 & -0.0642930 \\ \text { C } & 3.8563230 & 6.4212230 & -0.0487290 \\ \text { H } & 3.1578820 & 7.2619500 & -0.0410220 \\ \text { H } & 4.4834170 & 6.5047580 & 0.8463550 \\ \text { H } & 4.5102970 & 6.5207680 & -0.9189300 \\ \text { C } & 4.4293330 & -5.9485420 & 0.1241230 \\ \text { H } & 3.9850000 & -6.6931530 & 0.7943450 \\ \text { H } & 4.5105740 & -6.4101150 & -0.8647450 \\ \text { H } & 5.4376420 & -5.7332870 & 0.4865430\end{array}$

4 (excited-state/keto) G $=-1245.778879$ au

\begin{tabular}{|c|c|c|c|}
\hline C & 4.4667220 & -1.0920800 & -0.0036570 \\
\hline C & 4.5295210 & 0.3081520 & 0.0093580 \\
\hline C & 5.7155080 & 1.0096930 & 0.0222290 \\
\hline C & 6.8860790 & 0.2389890 & 0.0210550 \\
\hline C & 6.8381060 & -1.1571290 & 0.0075050 \\
\hline C & 5.6293390 & -1.8572820 & -0.0052450 \\
\hline C & 2.3975610 & -0.2474330 & -0.0066080 \\
\hline $\mathrm{H}$ & 5.5954810 & -2.9402730 & -0.0157710 \\
\hline$N$ & 3.1249500 & -1.3947180 & -0.0141180 \\
\hline $\mathrm{H}$ & 5.7314000 & 2.0929400 & 0.0326340 \\
\hline 0 & 3.2540020 & 0.8122700 & 0.0063990 \\
\hline $\mathrm{H}$ & 7.7669710 & -1.7178000 & 0.0067910 \\
\hline C & 0.9948930 & -0.0725160 & -0.0146190 \\
\hline C & 0.1430130 & -1.2837300 & -0.0061560 \\
\hline C & 0.4286190 & 1.1902250 & -0.0323890 \\
\hline C & -1.2892200 & -1.0816070 & 0.0060170 \\
\hline C & -0.9892250 & 1.3622450 & -0.0036540 \\
\hline $\mathrm{H}$ & 1.0751600 & 2.0613710 & -0.0167060 \\
\hline C & -1.8055470 & 0.2335230 & 0.0101730 \\
\hline $\mathrm{O}$ & 0.6904030 & -2.4118230 & -0.0365630 \\
\hline $\mathrm{H}$ & 7.8476870 & 0.7401430 & 0.0308290 \\
\hline C & -1.5582390 & 2.7158840 & 0.0233320 \\
\hline C & -2.7975350 & 2.9748320 & 0.6365580 \\
\hline C & -0.8860380 & 3.7980860 & -0.5670300 \\
\hline C & -3.3343900 & 4.2523010 & 0.6509390 \\
\hline $\mathrm{H}$ & -3.3266860 & 2.1681950 & 1.1343710 \\
\hline C & -1.4335970 & 5.0752440 & -0.5536870 \\
\hline $\mathrm{H}$ & 0.0585190 & 3.6319860 & -1.0759430 \\
\hline C & -2.6635280 & 5.3285600 & 0.0565690 \\
\hline
\end{tabular}




$\begin{array}{lrrr}\text { H } & -4.2895050 & 4.4258080 & 1.1407200 \\ \text { H } & -0.8979550 & 5.8910600 & -1.0321490 \\ \text { C } & -2.2007200 & -2.2263910 & -0.0235240 \\ \text { C } & -1.8835840 & -3.4647460 & 0.5740610 \\ \text { C } & -3.4656380 & -2.1128370 & -0.6335860 \\ \text { C } & -2.7879180 & -4.5150180 & 0.5603760 \\ \text { H } & -0.9255680 & -3.5845240 & 1.0623150 \\ \text { C } & -4.3596980 & -3.1742620 & -0.6489650 \\ \text { H } & -3.7359740 & -1.1892230 & -1.1364730 \\ \text { C } & -4.0411110 & -4.3968990 & -0.0526060 \\ \text { H } & -2.5208080 & -5.4521500 & 1.0438130 \\ \text { H } & -5.3221870 & -3.0552370 & -1.1407120 \\ \text { H } & -2.8815250 & 0.3661080 & 0.0310450 \\ \text { C } & -3.2489800 & 6.7154130 & 0.0978310 \\ \text { H } & -2.7215540 & 7.3893050 & -0.5817160 \\ \text { H } & -4.3069980 & 6.7055520 & -0.1811790 \\ \text { H } & -3.1826450 & 7.1372640 & 1.1068560 \\ \text { C } & -4.9985660 & -5.5586410 & -0.0835910 \\ \text { H } & -4.6852850 & -6.3057870 & -0.8219670 \\ \text { H } & -5.0423820 & -6.0615900 & 0.8873770 \\ \text { H } & -6.0085610 & -5.2341970 & -0.3472980 \\ \text { H } & 2.6275670 & -2.2849710 & -0.0308390\end{array}$

\begin{tabular}{|c|c|c|c|}
\hline \multicolumn{3}{|c|}{4 (excited-state/ESIPT TS) } & $=-1245.77$ \\
\hline C & 4.4851230 & -0.3301350 & -0.0043970 \\
\hline C & 4.4397210 & 1.0783590 & 0.0025560 \\
\hline C & 5.5616220 & 1.8780670 & 0.0016950 \\
\hline C & 6.7941330 & 1.2109550 & -0.0073900 \\
\hline C & 6.8631510 & -0.1864040 & -0.0152340 \\
\hline C & 5.7193860 & -0.9843180 & -0.0140200 \\
\hline C & 2.4025540 & 0.3152610 & 0.0085490 \\
\hline $\mathrm{H}$ & 5.7773350 & -2.0666390 & -0.0201510 \\
\hline $\mathrm{N}$ & 3.1899230 & -0.7761810 & -0.0004250 \\
\hline $\mathrm{H}$ & 5.4868670 & 2.9592110 & 0.0076930 \\
\hline $\mathrm{O}$ & 3.1251910 & 1.4748870 & 0.0095220 \\
\hline $\mathrm{H}$ & 7.8372530 & -0.6651310 & -0.0223970 \\
\hline C & 0.9962810 & 0.2675290 & 0.0034330 \\
\hline C & 0.3963770 & -1.0806430 & 0.0129080 \\
\hline C & 0.1648040 & 1.3821420 & -0.0123810 \\
\hline C & -1.0259330 & -1.2201970 & 0.0284470 \\
\hline C & -1.2481190 & 1.2338060 & 0.0141180 \\
\hline $\mathrm{H}$ & 0.6109390 & 2.3698660 & 0.0128070 \\
\hline C & -1.8146220 & -0.0493920 & 0.0318490 \\
\hline $\mathrm{O}$ & 1.1785720 & -2.1046680 & -0.0256950 \\
\hline $\mathrm{H}$ & 2.3104570 & -1.7233760 & -0.0212480 \\
\hline $\mathrm{H}$ & 7.7100090 & 1.7918930 & -0.0083660 \\
\hline C & -2.1025030 & 2.4258630 & 0.0346380 \\
\hline C & -3.3751780 & 2.4005750 & 0.6338780 \\
\hline C & -1.6761260 & 3.6340860 & -0.5440270 \\
\hline C & -4.1779260 & 3.5301330 & 0.6506680 \\
\hline $\mathrm{H}$ & -3.7174940 & 1.4960690 & 1.1263220 \\
\hline C & -2.4906660 & 4.7575450 & -0.5331380 \\
\hline $\mathrm{H}$ & -0.7104240 & 3.6828520 & -1.0369370 \\
\hline C & -3.7549810 & 4.7285440 & 0.0627870 \\
\hline $\mathrm{H}$ & -5.1493350 & 3.4894470 & 1.1365800 \\
\hline $\mathrm{H}$ & -2.1417730 & 5.6757990 & -0.9980170 \\
\hline C & -1.6576470 & -2.5420680 & -0.0135000 \\
\hline C & -1.1031140 & -3.6729560 & 0.6201650 \\
\hline C & -2.8914110 & -2.7077800 & -0.6707090 \\
\hline C & -1.7568250 & -4.8945500 & 0.5921350 \\
\hline $\mathrm{H}$ & -0.1658340 & -3.5793210 & 1.1538120 \\
\hline C & -3.5327480 & -3.9381100 & -0.7001100 \\
\hline
\end{tabular}




$\begin{array}{lrrr}\text { H } & -3.3327450 & -1.8665810 & -1.1966720 \\ \mathrm{C} & -2.9801980 & -5.0561130 & -0.0699510 \\ \mathrm{H} & -1.3137090 & -5.7461540 & 1.1030570 \\ \mathrm{H} & -4.4777360 & -4.0359830 & -1.2286310 \\ \mathrm{H} & -2.8927440 & -0.1521560 & 0.0475350 \\ \mathrm{C} & -4.6470240 & 5.9408600 & 0.0548880 \\ \mathrm{H} & -4.0664750 & 6.8603890 & -0.0539080 \\ \mathrm{H} & -5.3548790 & 5.8945080 & -0.7805670 \\ \mathrm{H} & -5.2321600 & 6.0076440 & 0.9761580 \\ \mathrm{C} & -3.6608560 & -6.3981620 & -0.1145320 \\ \mathrm{H} & -3.1568680 & -7.0688430 & -0.8198290 \\ \mathrm{H} & -3.6408990 & -6.8843580 & 0.8656050 \\ \mathrm{H} & -4.7029920 & -6.3049940 & -0.4304360\end{array}$


5 (ground-state) G= $-1435.032061 \mathrm{au}$

\begin{tabular}{|c|c|c|c|}
\hline C & -5.2891080 & -0.7661790 & -0.0939150 \\
\hline$C$ & -5.2593120 & 0.6109230 & 0.1306590 \\
\hline C & -6.3911660 & 1.3963000 & 0.2628350 \\
\hline C & -7.6046730 & 0.7209290 & 0.1573500 \\
\hline C & -7.6613870 & -0.6644060 & -0.0679270 \\
\hline C & -6.5094230 & -1.4320040 & -0.1975010 \\
\hline C & -3.2429460 & -0.1550530 & -0.0036260 \\
\hline $\mathrm{H}$ & -6.5484590 & -2.5014610 & -0.3714820 \\
\hline $\mathrm{N}$ & -3.9747630 & -1.2171710 & -0.1737320 \\
\hline $\mathrm{H}$ & -6.3316750 & 2.4642620 & 0.4362430 \\
\hline 0 & -3.9460470 & 0.9925660 & 0.1877390 \\
\hline $\mathrm{H}$ & -8.6309930 & -1.1454800 & -0.1424110 \\
\hline C & -1.7913640 & -0.0909180 & 0.0012330 \\
\hline C & -1.0477030 & -1.2728380 & -0.2089440 \\
\hline C & -1.1417940 & 1.1320200 & 0.2078570 \\
\hline C & 0.3620050 & -1.2065520 & -0.2032840 \\
\hline C & 0.2442540 & 1.2127600 & 0.2149330 \\
\hline $\mathrm{H}$ & -1.7416710 & 2.0272220 & 0.3383010 \\
\hline C & 0.9674760 & 0.0280270 & 0.0089320 \\
\hline $\mathrm{O}$ & -1.6297790 & -2.4665700 & -0.3986750 \\
\hline $\mathrm{H}$ & -2.6098470 & -2.3628680 & -0.3613600 \\
\hline $\mathrm{H}$ & -8.5293780 & 1.2803200 & 0.2518730 \\
\hline C & 0.9356600 & 2.5100840 & 0.4227730 \\
\hline C & 2.0954380 & 2.8023990 & -0.2979360 \\
\hline C & 0.4278390 & 3.4473990 & 1.3278500 \\
\hline C & 2.7822660 & 4.0199200 & -0.1289250 \\
\hline $\mathrm{H}$ & 2.4441670 & 2.0781380 & -1.0245290 \\
\hline C & 1.0870560 & 4.6610240 & 1.4878240 \\
\hline $\mathrm{H}$ & -0.4573190 & 3.2179750 & 1.9125610 \\
\hline C & 2.2420830 & 4.9567870 & 0.7755860 \\
\hline $\mathrm{H}$ & 0.6987360 & 5.3962950 & 2.1867270 \\
\hline C & 1.1986550 & -2.4211750 & -0.3986430 \\
\hline C & 0.9347720 & -3.3253150 & -1.4321570 \\
\hline C & 2.2998630 & -2.6232530 & 0.4357710 \\
\hline C & 1.7880170 & -4.4065940 & -1.6143700 \\
\hline $\mathrm{H}$ & 0.0820640 & -3.1740450 & -2.0831800 \\
\hline C & 3.1607060 & -3.7248880 & 0.2692320 \\
\hline $\mathrm{H}$ & 2.4689430 & -1.9161240 & 1.2394900 \\
\hline C & 2.8879540 & -4.6120580 & -0.7898980 \\
\hline $\mathrm{H}$ & 1.6014260 & -5.1073960 & -2.4230720 \\
\hline $\mathrm{H}$ & 2.0538120 & 0.0672820 & 0.0173640 \\
\hline $\mathrm{H}$ & 2.7208930 & 5.9165170 & 0.9251890 \\
\hline $\mathrm{H}$ & 3.5285990 & -5.4661960 & -0.9716290 \\
\hline$N$ & 4.2313460 & -3.9325250 & 1.1278860 \\
\hline $\mathrm{N}$ & 3.9480790 & 4.2877980 & -0.8289170 \\
\hline C & 4.4423140 & 5.6503190 & -0.8518670 \\
\hline $\mathrm{H}$ & 5.3383950 & 5.6914060 & -1.4718230 \\
\hline $\mathrm{H}$ & 3.7050480 & 6.3611610 & -1.2540790 \\
\hline $\mathrm{H}$ & 4.7211100 & 5.9796930 & 0.1542150 \\
\hline C & 4.3127580 & 3.4168470 & -1.9284660 \\
\hline $\mathrm{H}$ & 5.2430120 & 3.7752330 & -2.3703500 \\
\hline $\mathrm{H}$ & 4.4869450 & 2.3966530 & -1.5718960 \\
\hline $\mathrm{H}$ & 3.5430750 & 3.3788850 & -2.7139480 \\
\hline C & 5.2366870 & -4.9046790 & 0.7485540 \\
\hline $\mathrm{H}$ & 5.7022220 & -4.6780990 & -0.2227800 \\
\hline $\mathrm{H}$ & 6.0159010 & -4.9245360 & 1.5112130 \\
\hline $\mathrm{H}$ & 4.8032630 & -5.9083510 & 0.6931740 \\
\hline C & 4.6366480 & -2.8473680 & 1.9990150 \\
\hline $\mathrm{H}$ & 5.5139790 & -3.1580540 & 2.5672980 \\
\hline $\mathrm{H}$ & 4.8847580 & -1.9290750 & 1.4450960 \\
\hline
\end{tabular}


5 (ground-state/keto) G= $-1435.012188 \mathrm{au}$

\begin{tabular}{|c|c|c|c|}
\hline & 5.3362570 & -0.6422960 & 0.1042430 \\
\hline & 5.2940510 & 0.7317720 & -0.1231310 \\
\hline & 6.4229670 & 1.5157810 & -0.2417290 \\
\hline C & 7.6412750 & 0.8452870 & -0.1193300 \\
\hline$C$ & 7.6990120 & -0.5347640 & 0.1088740 \\
\hline C & 6.5466750 & -1.3107800 & 0.2260470 \\
\hline C & 3.2215460 & 0.0264040 & -0.0238260 \\
\hline $\mathrm{H}$ & 6.5901890 & -2.3789810 & 0.4025850 \\
\hline$N$ & 4.0067280 & -1.0406270 & 0.1588410 \\
\hline $\mathrm{H}$ & 6.3587060 & 2.5827280 & -0.4180350 \\
\hline O & 3.9753660 & 1.1271260 & -0.2005520 \\
\hline $\mathrm{H}$ & 8.6670830 & -1.0157340 & 0.1973590 \\
\hline C & 1.8194240 & 0.0327860 & -0.0352180 \\
\hline C & 1.1601880 & -1.2446530 & 0.1832220 \\
\hline C & 1.1059530 & 1.2411030 & -0.2353680 \\
\hline C & -0.2977930 & -1.1973870 & 0.1675410 \\
\hline C & -0.2653420 & 1.2489920 & -0.2331410 \\
\hline $\mathrm{H}$ & 1.6630470 & 2.1659540 & -0.3598140 \\
\hline C & -0.9336430 & 0.0034800 & -0.0266280 \\
\hline $\mathrm{O}$ & 1.8047180 & -2.3116790 & 0.3614840 \\
\hline $\mathrm{H}$ & 8.5636390 & 1.4092260 & -0.2035220 \\
\hline C & -1.0373450 & 2.5008280 & -0.4231010 \\
\hline C & -2.2189100 & 2.7080390 & 0.2933750 \\
\hline C & -0.5870230 & 3.4887350 & -1.3062230 \\
\hline C & -2.9791780 & 3.8834990 & 0.1444900 \\
\hline $\mathrm{H}$ & -2.5283890 & 1.9494270 & 1.0024000 \\
\hline C & -1.3176400 & 4.6631160 & -1.4438960 \\
\hline $\mathrm{H}$ & 0.3116070 & 3.3252050 & -1.8925830 \\
\hline C & -2.4935630 & 4.8724610 & -0.7344920 \\
\hline $\mathrm{H}$ & -0.9717310 & 5.4351890 & -2.1254310 \\
\hline C & -1.0846210 & -2.4447490 & 0.3408040 \\
\hline C & -0.7271790 & -3.4028160 & 1.2970280 \\
\hline C & -2.2307050 & -2.6425670 & -0.4345790 \\
\hline C & -1.5366110 & -4.5184120 & 1.4703100 \\
\hline $\mathrm{H}$ & 0.1676760 & -3.2652150 & 1.8903390 \\
\hline C & -3.0478110 & -3.7771120 & -0.2774750 \\
\hline $\mathrm{H}$ & -2.4688890 & -1.9032670 & -1.1902250 \\
\hline C & -2.6838760 & -4.7127700 & 0.7091340 \\
\hline $\mathrm{H}$ & -1.2759180 & -5.2586570 & 2.2216950 \\
\hline $\mathrm{H}$ & -2.0217700 & 0.0080680 & -0.0293170 \\
\hline $\mathrm{H}$ & -3.0308460 & 5.8030170 & -0.8688830 \\
\hline $\mathrm{H}$ & -3.2862490 & -5.5963170 & 0.8812860 \\
\hline$N$ & -4.1658080 & -3.9721820 & -1.0825230 \\
\hline $\mathrm{N}$ & -4.1664080 & 4.0618630 & 0.8401140 \\
\hline C & -4.7384840 & 5.3924510 & 0.8953870 \\
\hline $\mathrm{H}$ & -5.6395800 & 5.3653700 & 1.5090020 \\
\hline $\mathrm{H}$ & -4.0462430 & 6.1340770 & 1.3217850 \\
\hline $\mathrm{H}$ & -5.0294660 & 5.7321530 & -0.1037600 \\
\hline C & -4.4789020 & 3.1489190 & 1.9214150 \\
\hline $\mathrm{H}$ & -5.4338870 & 3.4364800 & 2.3625880 \\
\hline $\mathrm{H}$ & -4.5825580 & 2.1261430 & 1.5455430 \\
\hline $\mathrm{H}$ & -3.7133510 & 3.1472550 & 2.7120360 \\
\hline C & -5.1240370 & -4.9829420 & -0.6828020 \\
\hline $\mathrm{H}$ & -5.5310000 & -4.8103800 & 0.3256530 \\
\hline $\mathrm{H}$ & -5.9501730 & -4.9894210 & -1.3949400 \\
\hline $\mathrm{H}$ & -4.6666490 & -5.9771530 & -0.6998440 \\
\hline C & -4.6665590 & -2.8432560 & -1.8414410 \\
\hline $\mathrm{H}$ & -5.5720450 & -3.1448650 & -2.3694290 \\
\hline
\end{tabular}




$\begin{array}{llll}H & -4.9027050 & -1.9752940 & -1.2064710 \\ H & -3.9369500 & -2.5259830 & -2.5929910 \\ H & 3.5154180 & -1.9408690 & 0.3035650\end{array}$

5 (ground-state/PT TS) G= $-1435.013762 \mathrm{au}, v(\mathrm{imag})=583 i \mathrm{~cm}^{-1}$

\begin{tabular}{|c|c|c|c|}
\hline C & -5.3075260 & 0.4940040 & 0.1080990 \\
\hline C & -5.2819800 & -0.8807000 & -0.1290380 \\
\hline C & -6.4193750 & -1.6525790 & -0.2551430 \\
\hline C & -7.6294130 & -0.9695340 & -0.1303530 \\
\hline C & -7.6727440 & 0.4106270 & 0.1074110 \\
\hline $\mathrm{C}$ & -6.5133380 & 1.1728960 & 0.2320310 \\
\hline C & -3.2194940 & -0.1894070 & -0.0218690 \\
\hline $\mathrm{H}$ & -6.5451290 & 2.2403250 & 0.4156480 \\
\hline N & -3.9759500 & 0.8842580 & 0.1684620 \\
\hline $\mathrm{H}$ & -6.3669910 & -2.7189460 & -0.4389850 \\
\hline 0 & -3.9667430 & -1.2898850 & -0.2078280 \\
\hline $\mathrm{H}$ & -8.6363180 & 0.9004610 & 0.1970220 \\
\hline C & -1.8069360 & -0.1606810 & -0.0283510 \\
\hline C & -1.2098310 & 1.1366880 & 0.1958270 \\
\hline C & -1.0441160 & -1.3297410 & -0.2323270 \\
\hline C & 0.2362100 & 1.1695540 & 0.1816050 \\
\hline C & 0.3304630 & -1.2723540 & -0.2256650 \\
\hline $\mathrm{H}$ & -1.5579610 & -2.2782990 & -0.3649950 \\
\hline C & 0.9340950 & -0.0022920 & -0.0150680 \\
\hline $\mathrm{O}$ & -1.9170070 & 2.1837910 & 0.3806910 \\
\hline $\mathrm{H}$ & -3.3338870 & 1.7668520 & 0.3148790 \\
\hline $\mathrm{H}$ & -8.5580090 & -1.5223960 & -0.2201950 \\
\hline C & 1.1601530 & -2.4859680 & -0.4226390 \\
\hline C & 2.3427320 & -2.6487250 & 0.3032540 \\
\hline C & 0.7635720 & -3.4796650 & -1.3244460 \\
\hline C & 3.1582970 & -3.7851500 & 0.1440660 \\
\hline $\mathrm{H}$ & 2.6093470 & -1.8868250 & 1.0261220 \\
\hline C & 1.5500250 & -4.6160160 & -1.4735390 \\
\hline $\mathrm{H}$ & -0.1368930 & -3.3492890 & -1.9163280 \\
\hline C & 2.7279820 & -4.7809810 & -0.7559380 \\
\hline $\mathrm{H}$ & 1.2471300 & -5.3925260 & -2.1703030 \\
\hline C & 0.9618680 & 2.4545430 & 0.3515760 \\
\hline C & 0.5629380 & 3.3961280 & 1.3073450 \\
\hline C & 2.0930700 & 2.7049420 & -0.4301280 \\
\hline C & 1.3175760 & 4.5508650 & 1.4727270 \\
\hline $\mathrm{H}$ & -0.3203540 & 3.2157640 & 1.9067180 \\
\hline C & 2.8545310 & 3.8785760 & -0.2807280 \\
\hline $\mathrm{H}$ & 2.3635670 & 1.9757910 & -1.1847370 \\
\hline C & 2.4499660 & 4.7985560 & 0.7048670 \\
\hline $\mathrm{H}$ & 1.0248740 & 5.2797970 & 2.2233450 \\
\hline $\mathrm{H}$ & 2.0208540 & 0.0488370 & -0.0165260 \\
\hline $\mathrm{H}$ & 3.3096470 & -5.6830630 & -0.8994650 \\
\hline $\mathrm{H}$ & 3.0092830 & 5.7110470 & 0.8711620 \\
\hline$N$ & 3.9577970 & 4.1248910 & -1.0913580 \\
\hline$N$ & 4.3450500 & -3.9198320 & 0.8497000 \\
\hline C & 4.9802450 & -5.2220930 & 0.8869570 \\
\hline $\mathrm{H}$ & 5.8720320 & -5.1633980 & 1.5118040 \\
\hline $\mathrm{H}$ & 4.3200620 & -6.0042280 & 1.2911060 \\
\hline $\mathrm{H}$ & 5.2984620 & -5.5280590 & -0.1148020 \\
\hline C & 4.6039550 & -3.0114340 & 1.9489110 \\
\hline $\mathrm{H}$ & 5.5657420 & -3.2631280 & 2.3971260 \\
\hline $\mathrm{H}$ & 4.6658320 & -1.9789960 & 1.5905840 \\
\hline $\mathrm{H}$ & 3.8300800 & -3.0571320 & 2.7300040 \\
\hline C & 4.8670390 & 5.1833470 & -0.7005410 \\
\hline $\mathrm{H}$ & 5.2866180 & 5.0351740 & 0.3065760 \\
\hline
\end{tabular}




$\begin{array}{llll}\mathrm{H} & 5.6885200 & 5.2273180 & -1.4166800 \\ \mathrm{H} & 4.3612690 & 6.1538030 & -0.7193200 \\ \mathrm{C} & 4.5081820 & 3.0198120 & -1.8509650 \\ \mathrm{H} & 5.3954530 & 3.3633690 & -2.3841220 \\ \mathrm{H} & 4.7888810 & 2.1655150 & -1.2156550 \\ \mathrm{H} & 3.7907360 & 2.6664620 & -2.5981390\end{array}$

\section{5 (excited-state/enol) G $=-1434.900466 \mathrm{au}$}

\begin{tabular}{|c|c|c|c|}
\hline C & 5.3269960 & -0.6168670 & 0.0853150 \\
\hline & 5.3164330 & 0.7811760 & -0.0965280 \\
\hline & 6.4547440 & 1.5525330 & -0.1923820 \\
\hline C & 7.6730740 & 0.8664840 & -0.0984940 \\
\hline$C$ & 7.7123800 & -0.5219250 & 0.0815100 \\
\hline C & 6.5522930 & -1.2876760 & 0.1764350 \\
\hline$C$ & 3.2746910 & 0.0407130 & -0.0072880 \\
\hline H & 6.5866640 & -2.3623630 & 0.3156470 \\
\hline $\mathrm{N}$ & 4.0312610 & -1.0549300 & 0.1388470 \\
\hline $\mathrm{H}$ & 6.4034710 & 2.6261910 & -0.3320490 \\
\hline $\mathrm{O}$ & 4.0124160 & 1.1921150 & -0.1558210 \\
\hline H & 8.6767410 & -1.0158990 & 0.1491180 \\
\hline C & 1.8670810 & 0.0874630 & -0.0267330 \\
\hline C & 1.1340720 & -1.1685260 & 0.1405470 \\
\hline C & 1.1297740 & 1.2632540 & -0.2063310 \\
\hline C & -0.2854410 & -1.1907620 & 0.1656220 \\
\hline C & -0.2841890 & 1.2466630 & -0.1792130 \\
\hline $\mathrm{H}$ & 1.6633010 & 2.2010510 & -0.3051620 \\
\hline C & -0.9709090 & 0.0321430 & -0.0002700 \\
\hline $\mathrm{O}$ & 1.8043720 & -2.2924610 & 0.2200520 \\
\hline $\mathrm{H}$ & 2.8118420 & -2.0942190 & 0.2086640 \\
\hline $\mathrm{H}$ & 8.6013580 & 1.4235290 & -0.1672580 \\
\hline C & -1.0270310 & 2.5055650 & -0.3243550 \\
\hline C & -2.2816500 & 2.6613160 & 0.2800590 \\
\hline C & -0.4876730 & 3.5625620 & -1.0800400 \\
\hline C & -3.0249030 & 3.8452520 & 0.1416980 \\
\hline $\mathrm{H}$ & -2.6538910 & 1.8588380 & 0.9035300 \\
\hline C & -1.2144380 & 4.7372110 & -1.2212480 \\
\hline $\mathrm{H}$ & 0.4672150 & 3.4462140 & -1.5796080 \\
\hline C & -2.4573950 & 4.8922050 & -0.6218390 \\
\hline $\mathrm{H}$ & -0.8087560 & 5.5540110 & -1.8101890 \\
\hline C & -1.0280920 & -2.4512900 & 0.3018780 \\
\hline C & -0.5861840 & -3.4864100 & 1.1484770 \\
\hline C & -2.2476240 & -2.5972250 & -0.3758870 \\
\hline C & -1.3676230 & -4.6240810 & 1.2942750 \\
\hline $\mathrm{H}$ & 0.3369160 & -3.3810030 & 1.7030030 \\
\hline C & -3.0327760 & -3.7553800 & -0.2491980 \\
\hline $\mathrm{H}$ & -2.5578150 & -1.8055800 & -1.0464200 \\
\hline C & -2.5723120 & -4.7720280 & 0.6170080 \\
\hline $\mathrm{H}$ & -1.0395040 & -5.4163720 & 1.9605540 \\
\hline $\mathrm{H}$ & -2.0536020 & 0.0258330 & 0.0087830 \\
\hline $\mathrm{H}$ & -2.9872770 & 5.8286320 & -0.7459270 \\
\hline $\mathrm{H}$ & -3.1522950 & -5.6748890 & 0.7636580 \\
\hline$N$ & -4.2109050 & -3.9002480 & -0.9563590 \\
\hline$N$ & -4.2652490 & 3.9872280 & 0.7283870 \\
\hline C & -4.8763420 & 5.3010670 & 0.7818810 \\
\hline $\mathrm{H}$ & -5.8238790 & 5.2284340 & 1.3157060 \\
\hline $\mathrm{H}$ & -4.2423900 & 6.0366670 & 1.2969210 \\
\hline $\mathrm{H}$ & -5.0902400 & 5.6762620 & -0.2246210 \\
\hline C & -4.7289720 & 2.9652420 & 1.6443820 \\
\hline $\mathrm{H}$ & -5.7258310 & 3.2302030 & 1.9968540 \\
\hline $\mathrm{H}$ & -4.7995700 & 1.9959630 & 1.1390970 \\
\hline
\end{tabular}




$\begin{array}{rrrr}\text { H } & -4.0681830 & 2.8499300 & 2.5158810 \\ \text { C } & -5.0865350 & -5.0127700 & -0.6493820 \\ \text { H } & -5.4286080 & -5.0046570 & 0.3959040 \\ \text { H } & -5.9603380 & -4.9655410 & -1.2995360 \\ \text { H } & -4.5841090 & -5.9674250 & -0.8375450 \\ \text { C } & -4.7585780 & -2.7507070 & -1.6461950 \\ \text { H } & -5.7094570 & -3.0282940 & -2.1012710 \\ \text { H } & -4.9298110 & -1.9007190 & -0.9689920 \\ \text { H } & -4.0892530 & -2.4175980 & -2.4469400\end{array}$

5 (excited-state/keto) G $=-1434.909078 \mathrm{au}$

\begin{tabular}{|c|c|c|c|}
\hline C & 5.4031310 & -0.7330550 & 0.0878480 \\
\hline C & 5.3408010 & 0.6589780 & -0.0662500 \\
\hline C & 6.4596300 & 1.4607460 & -0.1310820 \\
\hline C & 7.6942260 & 0.8038420 & -0.0336760 \\
\hline C & 7.7706690 & -0.5824310 & 0.1197080 \\
\hline C & 6.6290210 & -1.3851590 & 0.1841780 \\
\hline C & 3.2671410 & -0.0852520 & -0.0261920 \\
\hline $\mathrm{H}$ & 6.6911410 & -2.4604120 & 0.3030400 \\
\hline $\mathrm{N}$ & 4.0945530 & -1.1548350 & 0.1062650 \\
\hline $\mathrm{H}$ & 6.3792720 & 2.5345730 & -0.2501500 \\
\hline 0 & 4.0257260 & 1.0419110 & -0.1361320 \\
\hline $\mathrm{H}$ & 8.7455280 & -1.0533590 & 0.1911630 \\
\hline C & 1.8546650 & -0.0415640 & -0.0534450 \\
\hline C & 1.1174010 & -1.3166260 & 0.1053870 \\
\hline C & 1.1749980 & 1.1512440 & -0.2316920 \\
\hline C & -0.3267680 & -1.2452800 & 0.1066600 \\
\hline C & -0.2522120 & 1.1950820 & -0.2128760 \\
\hline $\mathrm{H}$ & 1.7372650 & 2.0734060 & -0.3355760 \\
\hline C & -0.9610080 & 0.0070410 & -0.0511780 \\
\hline $\mathrm{O}$ & 1.7664690 & -2.3860550 & 0.1991750 \\
\hline $\mathrm{H}$ & 8.6071720 & 1.3873260 & -0.0789020 \\
\hline C & -0.9435360 & 2.4862590 & -0.3525730 \\
\hline C & -2.1830900 & 2.6939690 & 0.2671330 \\
\hline C & -0.3723070 & 3.5217470 & -1.1095220 \\
\hline C & -2.8834890 & 3.9048780 & 0.1342750 \\
\hline $\mathrm{H}$ & -2.5789870 & 1.9061040 & 0.8951360 \\
\hline C & -1.0522050 & 4.7263830 & -1.2374240 \\
\hline $\mathrm{H}$ & 0.5725610 & 3.3710370 & -1.6201170 \\
\hline C & -2.2829280 & 4.9312470 & -0.6275970 \\
\hline $\mathrm{H}$ & -0.6178590 & 5.5288940 & -1.8262800 \\
\hline C & -1.1315070 & -2.4633780 & 0.2426600 \\
\hline C & -0.7042370 & -3.5517450 & 1.0280320 \\
\hline C & -2.3884520 & -2.5347690 & -0.3834030 \\
\hline C & -1.5350930 & -4.6528680 & 1.1739120 \\
\hline $\mathrm{H}$ & 0.2536330 & -3.5116820 & 1.5277520 \\
\hline C & -3.2243050 & -3.6552000 & -0.2566340 \\
\hline $\mathrm{H}$ & -2.6908540 & -1.7121300 & -1.0189080 \\
\hline C & -2.7783560 & -4.7198290 & 0.5536130 \\
\hline $\mathrm{H}$ & -1.2159630 & -5.4839590 & 1.7965950 \\
\hline $\mathrm{H}$ & -2.0448020 & 0.0424070 & -0.0378840 \\
\hline $\mathrm{H}$ & -2.7733420 & 5.8900660 & -0.7415600 \\
\hline $\mathrm{H}$ & -3.3948900 & -5.5979660 & 0.7012510 \\
\hline$N$ & -4.4435490 & -3.7186160 & -0.9200280 \\
\hline$N$ & -4.1188080 & 4.0887570 & 0.7307330 \\
\hline C & -4.6563650 & 5.4322520 & 0.8193960 \\
\hline $\mathrm{H}$ & -5.6071420 & 5.3981310 & 1.3519040 \\
\hline $\mathrm{H}$ & -3.9833770 & 6.1219030 & 1.3498140 \\
\hline $\mathrm{H}$ & -4.8502000 & 5.8417080 & -0.1774100 \\
\hline C & -4.5849350 & 3.1021950 & 1.6841130 \\
\hline
\end{tabular}




$\begin{array}{rrrr}\text { H } & -5.5687440 & 3.3990640 & 2.0486210 \\ \text { H } & -4.6892770 & 2.1226730 & 1.2060380 \\ \text { H } & -3.9097430 & 2.9925930 & 2.5458610 \\ \text { C } & -5.3825340 & -4.7530050 & -0.5346600 \\ \text { H } & -5.6560350 & -4.7055650 & 0.5305690 \\ \text { H } & -6.2894090 & -4.6478110 & -1.1312790 \\ \text { H } & -4.9687650 & -5.7459440 & -0.7368120 \\ \text { C } & -4.9831990 & -2.4958350 & -1.4799380 \\ \text { H } & -5.9666860 & -2.7030790 & -1.9031240 \\ \text { H } & -5.0848560 & -1.6960620 & -0.7299980 \\ \text { H } & -4.3467530 & -2.1235070 & -2.2891720 \\ \text { H } & 3.6772210 & -2.0816080 & 0.1936660\end{array}$

5 (excited-state/ESIPT TS) G= $-1434.900892 \mathrm{au}, v($ imag $)=1149 i \mathrm{~cm}^{-1}$

\begin{tabular}{|c|c|c|c|}
\hline C & 5.3207610 & -0.4687080 & 0.0875260 \\
\hline$C$ & 5.3249220 & 0.9295610 & -0.0888050 \\
\hline C & 6.4742810 & 1.6840690 & -0.1755790 \\
\hline C & 7.6831850 & 0.9807890 & -0.0783500 \\
\hline C & 7.7028350 & -0.4068680 & 0.0960570 \\
\hline C & 6.5314010 & -1.1592220 & 0.1824680 \\
\hline C & 3.2611910 & 0.2427890 & -0.0164440 \\
\hline $\mathrm{H}$ & 6.5513990 & -2.2344980 & 0.3174030 \\
\hline $\mathrm{N}$ & 4.0108140 & -0.8663940 & 0.1285610 \\
\hline $\mathrm{H}$ & 6.4374420 & 2.7586890 & -0.3111860 \\
\hline $\mathrm{O}$ & 4.0254010 & 1.3677340 & -0.1543650 \\
\hline $\mathrm{H}$ & 8.6593660 & -0.9150580 & 0.1665310 \\
\hline C & 1.8545090 & 0.2407850 & -0.0366670 \\
\hline C & 1.2086760 & -1.0748680 & 0.1374870 \\
\hline C & 1.0628240 & 1.3706390 & -0.2140880 \\
\hline C & -0.2173210 & -1.1615400 & 0.1509360 \\
\hline C & -0.3540180 & 1.2750750 & -0.1879790 \\
\hline $\mathrm{H}$ & 1.5416620 & 2.3380790 & -0.3154410 \\
\hline C & -0.9642880 & 0.0250460 & -0.0139660 \\
\hline $\mathrm{O}$ & 1.9559620 & -2.1208540 & 0.2360850 \\
\hline $\mathrm{H}$ & 3.1058580 & -1.7761390 & 0.2131140 \\
\hline $\mathrm{H}$ & 8.6188350 & 1.5258400 & -0.1403680 \\
\hline C & -1.1659050 & 2.4917680 & -0.3339470 \\
\hline C & -2.4233530 & 2.5811130 & 0.2782610 \\
\hline C & -0.6902460 & 3.5731000 & -1.0952080 \\
\hline C & -3.2327580 & 3.7208840 & 0.1386890 \\
\hline $\mathrm{H}$ & -2.7470030 & 1.7615850 & 0.9068360 \\
\hline C & -1.4815540 & 4.7056170 & -1.2352610 \\
\hline $\mathrm{H}$ & 0.2670420 & 3.5087930 & -1.5999740 \\
\hline C & -2.7281240 & 4.7945870 & -0.6298220 \\
\hline $\mathrm{H}$ & -1.1241090 & 5.5419290 & -1.8282860 \\
\hline C & -0.8963270 & -2.4562310 & 0.2841620 \\
\hline C & -0.3883410 & -3.4813930 & 1.1047350 \\
\hline C & -2.1183170 & -2.6559800 & -0.3778810 \\
\hline C & -1.1102490 & -4.6582160 & 1.2446410 \\
\hline $\mathrm{H}$ & 0.5403720 & -3.3389990 & 1.6406560 \\
\hline C & -2.8422410 & -3.8533970 & -0.2580380 \\
\hline $\mathrm{H}$ & -2.4787150 & -1.8729730 & -1.0331170 \\
\hline C & -2.3180560 & -4.8568560 & 0.5851640 \\
\hline $\mathrm{H}$ & -0.7316760 & -5.4436040 & 1.8923290 \\
\hline $\mathrm{H}$ & -2.0456310 & -0.0386390 & -0.0032090 \\
\hline $\mathrm{H}$ & -3.3082940 & 5.7008070 & -0.7527830 \\
\hline $\mathrm{H}$ & -2.8488800 & -5.7901460 & 0.7274450 \\
\hline$N$ & -4.0237440 & -4.0479310 & -0.9537340 \\
\hline$N$ & -4.4785200 & 3.7935960 & 0.7311120 \\
\hline C & -5.1524290 & 5.0758120 & 0.7951070 \\
\hline
\end{tabular}




$\begin{array}{lrrr}\mathrm{H} & -6.0951930 & 4.9531270 & 1.3285050 \\ \mathrm{H} & -4.5549950 & 5.8390760 & 1.3142480 \\ \mathrm{H} & -5.3855350 & 5.4462420 & -0.2086630 \\ \mathrm{C} & -4.8661110 & 2.7619460 & 1.6716950 \\ \mathrm{H} & -5.8731710 & 2.9714110 & 2.0326940 \\ \mathrm{H} & -4.8837940 & 1.7813060 & 1.1844040 \\ \mathrm{H} & -4.1888750 & 2.7028670 & 2.5364170 \\ \mathrm{C} & -4.8527180 & -5.1873350 & -0.6158070 \\ \mathrm{H} & -5.1697780 & -5.1835150 & 0.4377780 \\ \mathrm{H} & -5.7427610 & -5.1768880 & -1.2455560 \\ \mathrm{H} & -4.3216600 & -6.1249730 & -0.8094850 \\ \mathrm{C} & -4.6500990 & -2.9066480 & -1.5889640 \\ \mathrm{H} & -5.5910820 & -3.2227990 & -2.0398200 \\ \mathrm{H} & -4.8578950 & -2.0920910 & -0.8785810 \\ \mathrm{H} & -4.0164620 & -2.5072800 & -2.3879570\end{array}$


6 (ground-state) G= $-1592.105904 \mathrm{au}$

$\begin{array}{lrrr}\mathrm{C} & 0.0559710 & 5.6430660 & -0.0229590 \\ \mathrm{C} & -1.3232900 & 5.4305430 & -0.0042750 \\ \mathrm{C} & -2.2611320 & 6.4482130 & 0.0014940 \\ \mathrm{C} & -1.7431720 & 7.7411650 & -0.0133510 \\ \mathrm{C} & -0.3594620 & 7.9814350 & -0.0327520 \\ \mathrm{C} & 0.5633330 & 6.9413260 & -0.0378460 \\ \mathrm{C} & -0.2873260 & 3.5328810 & -0.0042250 \\ \mathrm{H} & 1.6322830 & 7.1222190 & -0.0527150 \\ \mathrm{~N} & 0.6825750 & 4.4004930 & -0.0221190 \\ \mathrm{H} & -3.3258490 & 6.2472950 & 0.0169170 \\ \mathrm{O} & -1.5333420 & 4.0783300 & 0.0067820 \\ \mathrm{H} & -0.0040930 & 9.0065090 & -0.0438570 \\ \mathrm{C} & -0.1619450 & 2.0856240 & 0.0025130 \\ \mathrm{C} & 1.1238200 & 1.5044280 & 0.0031990 \\ \mathrm{C} & -1.3095470 & 1.2816670 & 0.0119010 \\ \mathrm{C} & 1.2438910 & 0.0970270 & 0.0118890 \\ \mathrm{C} & -1.2112730 & -0.1035520 & 0.0145030 \\ \mathrm{H} & -2.2824930 & 1.7618130 & 0.0435470 \\ \mathrm{C} & 0.0769450 & -0.6625630 & 0.0162700 \\ \mathrm{O} & 2.2484210 & 2.2406170 & -0.0194050 \\ \mathrm{H} & 2.0120110 & 3.1972900 & -0.0409010 \\ \mathrm{H}\end{array}$




$\begin{array}{llll}\mathrm{C} & 6.0580090 & -5.0795880 & -0.4627290 \\ \mathrm{H} & 7.6062850 & -3.7457090 & -1.1655940 \\ \mathrm{H} & 6.0619090 & -3.4907860 & -1.9457630 \\ \mathrm{H} & 6.2714020 & -5.8695730 & -1.1892000 \\ \mathrm{H} & 6.5690530 & -5.3257200 & 0.4733250 \\ \mathrm{H} & 4.9823490 & -5.0699560 & -0.2647150 \\ \mathrm{C} & 7.3483440 & -2.2705690 & 0.8494400 \\ \mathrm{C} & 8.2309470 & -1.1491080 & 0.3017760 \\ \mathrm{H} & 7.9518910 & -3.1688760 & 1.0200790 \\ \mathrm{H} & 6.9326320 & -1.9975240 & 1.8246210 \\ \mathrm{H} & 9.0330100 & -0.9043860 & 1.0047200 \\ \mathrm{H} & 8.6855070 & -1.4524760 & -0.6465720 \\ \mathrm{H} & 7.6417380 & -0.2463250 & 0.1173630\end{array}$

6 (ground-state/keto) G= $-1592.085500 \mathrm{au}$

\begin{tabular}{|c|c|c|c|}
\hline$c$ & 0.4688240 & 5.6450280 & -0.0008270 \\
\hline C & -0.9219630 & 5.5560940 & -0.0139950 \\
\hline C & -1.7518350 & 6.6578230 & -0.0298610 \\
\hline C & -1.1122400 & 7.8989660 & -0.0324430 \\
\hline C & 0.2834130 & 8.0036630 & -0.0196290 \\
\hline C & 1.1064430 & 6.8779050 & -0.0033950 \\
\hline C & -0.1409650 & 3.5072890 & 0.0074280 \\
\hline $\mathrm{H}$ & 2.1870740 & 6.9581210 & 0.0064130 \\
\hline $\mathrm{N}$ & 0.9151620 & 4.3303270 & 0.0136050 \\
\hline $\mathrm{H}$ & -2.8304390 & 6.5569260 & -0.0396660 \\
\hline 0 & -1.2809600 & 4.2252930 & -0.0090560 \\
\hline $\mathrm{H}$ & 0.7396810 & 8.9876540 & -0.0223760 \\
\hline C & -0.1046900 & 2.1077810 & 0.0135150 \\
\hline C & 1.2105450 & 1.4906360 & 0.0328200 \\
\hline C & -1.3084330 & 1.3545410 & 0.0119870 \\
\hline C & 1.2127590 & 0.0303380 & 0.0300420 \\
\hline C & -1.2707620 & -0.0153440 & 0.0202560 \\
\hline $\mathrm{H}$ & -2.2577310 & 1.8830770 & 0.0344530 \\
\hline C & 0.0149240 & -0.6415470 & 0.0334030 \\
\hline 0 & 2.2716460 & 2.1712540 & 0.0408640 \\
\hline $\mathrm{H}$ & -1.7133110 & 8.8014390 & -0.0448320 \\
\hline C & -2.5047450 & -0.8324620 & 0.0239980 \\
\hline C & -2.5911120 & -2.0348020 & 0.7346360 \\
\hline C & -3.6482940 & -0.4352990 & -0.6779730 \\
\hline C & -3.7477900 & -2.8010360 & 0.7498920 \\
\hline $\mathrm{H}$ & -1.7348860 & -2.3797020 & 1.3088510 \\
\hline C & -4.8180870 & -1.1805260 & -0.6657550 \\
\hline $\mathrm{H}$ & -3.6198910 & 0.4788170 & -1.2657690 \\
\hline C & -4.9036830 & -2.3935910 & 0.0505270 \\
\hline $\mathrm{H}$ & -3.7440780 & -3.7287460 & 1.3100220 \\
\hline $\mathrm{H}$ & -5.6717770 & -0.8100250 & -1.2211430 \\
\hline C & 2.4932730 & -0.7111140 & -0.0146710 \\
\hline C & 3.6303120 & -0.2972750 & 0.6923280 \\
\hline C & 2.6094300 & -1.8951210 & -0.7523660 \\
\hline C & 4.8063700 & -1.0317530 & 0.6800140 \\
\hline $\mathrm{H}$ & 3.5923020 & 0.6253730 & 1.2587820 \\
\hline C & 3.7769910 & -2.6440900 & -0.7744160 \\
\hline $\mathrm{H}$ & 1.7635310 & -2.2391470 & -1.3423530 \\
\hline C & 4.9167400 & -2.2335760 & -0.0520620 \\
\hline $\mathrm{H}$ & 5.6545610 & -0.6519090 & 1.2377510 \\
\hline $\mathrm{H}$ & 3.7944150 & -3.5570890 & -1.3581460 \\
\hline $\mathrm{H}$ & 0.0426110 & -1.7293340 & 0.0393580 \\
\hline $\mathrm{N}$ & -6.0616850 & -3.1462710 & 0.0636790 \\
\hline$N$ & 6.0861890 & -2.9688410 & -0.0655990 \\
\hline C & -6.1933780 & -4.3216990 & 0.9058900 \\
\hline
\end{tabular}




$\begin{array}{llll}\text { C } & -5.6561500 & -5.5935600 & 0.2495440 \\ \text { H } & -7.2574910 & -4.4442800 & 1.1362030 \\ \text { H } & -5.6972490 & -4.1400030 & 1.8648400 \\ \text { H } & -5.7748650 & -6.4558720 & 0.9126540 \\ \text { H } & -6.1959860 & -5.7978910 & -0.6804310 \\ \text { H } & -4.5956030 & -5.4861280 & 0.0045650 \\ \text { C } & -7.2001770 & -2.8017160 & -0.7689340 \\ \text { C } & -8.1355980 & -1.7843520 & -0.1154110 \\ \text { H } & -7.7475380 & -3.7274720 & -0.9780260 \\ \text { H } & -6.8456040 & -2.4364720 & -1.7384040 \\ \text { H } & -8.9801540 & -1.5519890 & -0.7713140 \\ \text { H } & -8.5296780 & -2.1807190 & 0.8256130 \\ \text { H } & -7.6032890 & -0.8556190 & 0.1092150 \\ \text { C } & 6.2487880 & -4.1197950 & -0.9352460 \\ \text { C } & 5.7242900 & -5.4165100 & -0.3181720 \\ \text { H } & 7.3177190 & -4.2196560 & -1.1543300 \\ \text { H } & 5.7619010 & -3.9225270 & -1.8959310 \\ \text { H } & 5.8647520 & -6.2598600 & -1.0012570 \\ \text { H } & 6.2558460 & -5.6358910 & 0.6131800 \\ \text { H } & 4.6592810 & -5.3313990 & -0.0840580 \\ \text { C } & 7.2136190 & -2.6182460 & 0.7791700 \\ \text { C } & 8.1402580 & -1.5811980 & 0.1443010 \\ \text { H } & 7.7716730 & -3.5390260 & 0.9828290 \\ \text { H } & 6.8460260 & -2.2679680 & 1.7490930 \\ \text { H } & 8.9773470 & -1.3457840 & 0.8087230 \\ \text { H } & 8.5460050 & -1.9611500 & -0.7986300 \\ \text { H } & 7.5969370 & -0.6571730 & -0.0728020 \\ \text { H } & 1.8422790 & 3.8698500 & 0.0230920\end{array}$

6 (ground-state/PT TS) G= $-1592.0868800 \mathrm{au}, v(\mathrm{imag})=561 i \mathrm{~cm}^{-1}$

$\begin{array}{lrrr}\text { C } & 0.8746690 & 5.5530490 & -0.0112490 \\ \text { C } & -0.5190890 & 5.6213260 & -0.0113010 \\ \text { C } & -1.2227890 & 6.8085650 & -0.0192640 \\ \text { C } & -0.4493970 & 7.9698970 & -0.0278780 \\ \text { C } & 0.9504980 & 7.9190960 & -0.0282890 \\ \text { C } & 1.6434110 & 6.7101580 & -0.0198870 \\ \text { C } & 0.0394650 & 3.5149800 & 0.0044250 \\ \text { H } & 2.7262790 & 6.6695560 & -0.0201570 \\ \text { N } & 1.1790280 & 4.1983810 & -0.0006650 \\ \text { H } & -2.3060300 & 6.8285530 & -0.0186000 \\ \text { O } & -1.0237890 & 4.3377240 & -0.0021410 \\ \text { H } & 1.5120720 & 8.8470330 & -0.0352760 \\ \text { C } & -0.0308800 & 2.1061510 & 0.0137420 \\ \text { C } & 1.2404120 & 1.4202180 & 0.0252780 \\ \text { C } & -1.2701310 & 1.4264270 & 0.0203900 \\ \text { C } & 1.1730450 & -0.0262920 & 0.0347870 \\ \text { C } & -1.3076210 & 0.0519480 & 0.0322820 \\ \text { H } & -2.1882480 & 2.0076000 & 0.0440630 \\ \text { C } & -0.0622090 & -0.6378690 & 0.0440680 \\ \text { O } & 2.3516190 & 2.0518870 & 0.0167680 \\ \text { H } & 2.0278240 & 3.5013000 & 0.0022820 \\ \text { H } & -0.9462040 & 8.9338320 & -0.0344010 \\ \text { C } & -2.5850660 & -0.6958070 & 0.0387140 \\ \text { C } & -2.7367330 & -1.8907150 & 0.7508580 \\ \text { C } & -3.7038970 & -0.2388020 & -0.6663340 \\ \text { C } & -3.9316000 & -2.5957020 & 0.7617530 \\ \text { H } & -1.9016840 & -2.2787520 & 1.3287200 \\ \text { C } & -4.9108970 & -0.9221990 & -0.6585110 \\ \text { H } & -3.6263020 & 0.6728080 & -1.2536430 \\ \text { C } & -5.0616440 & -2.1296970 & 0.0565060\end{array}$




\begin{tabular}{|c|c|c|c|}
\hline $\mathrm{H}$ & -3.9786540 & -3.5216000 & 1.3229200 \\
\hline $\mathrm{H}$ & -5.7424200 & -0.5074470 & -1.2161920 \\
\hline C & 2.4121070 & -0.8356930 & -0.0060820 \\
\hline C & 3.5643760 & -0.4866890 & 0.7103440 \\
\hline C & 2.4684460 & -2.0196050 & -0.7500600 \\
\hline C & 4.7014230 & -1.2803000 & 0.6970170 \\
\hline $\mathrm{H}$ & 3.5712000 & 0.4324950 & 1.2842770 \\
\hline C & 3.5962670 & -2.8272620 & -0.7733920 \\
\hline $\mathrm{H}$ & 1.6075580 & -2.3159210 & -1.3442590 \\
\hline C & 4.7532890 & -2.4798530 & -0.0454250 \\
\hline $\mathrm{H}$ & 5.5655600 & -0.9490400 & 1.2611660 \\
\hline $\mathrm{H}$ & 3.5687530 & -3.7363880 & 6700 \\
\hline $\mathrm{H}$ & -0.0879870 & -1.7254940 & 0.0545570 \\
\hline $\mathrm{N}$ & -6.2565960 & -2.8218820 & 0.0639150 \\
\hline $\mathrm{N}$ & 5.8842480 & -3.2727540 & -0.0629720 \\
\hline C & -6.4493000 & -3.9945370 & 0.8982740 \\
\hline C & -5.9681730 & -5.2866810 & 0.2379310 \\
\hline $\mathrm{H}$ & -7.5195200 & -4.0677690 & 1.1209410 \\
\hline $\mathrm{H}$ & -5.9517460 & -3.8417690 & 1.8614840 \\
\hline $\mathrm{H}$ & -6.1309330 & -6.1459700 & 0.8955930 \\
\hline $\mathrm{H}$ & -6.5114120 & -5.4607630 & -0.6961990 \\
\hline $\mathrm{H}$ & -4.9023180 & -5.2275640 & -0.0003700 \\
\hline C & -7.3698670 & -2.4229370 & -0.7786660 \\
\hline C & -8.2611780 & -1.3624170 & -0.1323050 \\
\hline $\mathrm{H}$ & -7.9591550 & -3.3212420 & -0.9934390 \\
\hline $\mathrm{H}$ & -6.9896870 & -2.0745660 & -1.7446350 \\
\hline $\mathrm{H}$ & -9.0883780 & -1.0903000 & -0.7949800 \\
\hline $\mathrm{H}$ & -8.6811260 & -1.7398970 & 0.8052710 \\
\hline $\mathrm{H}$ & -7.6873920 & -0.4599370 & 0.0971100 \\
\hline C & 5.9926270 & -4.4197050 & -0.9464120 \\
\hline C & 5.4055730 & -5.6967530 & -0.3450940 \\
\hline $\mathrm{H}$ & 7.0558200 & -4.5682990 & -1.1657100 \\
\hline $\mathrm{H}$ & 5.5170550 & -4.1877740 & -1.9050340 \\
\hline $\mathrm{H}$ & 5.5076570 & -6.5381460 & -1.0373260 \\
\hline $\mathrm{H}$ & 5.9236740 & -5.9512220 & 0.5848920 \\
\hline $\mathrm{H}$ & 4.3451860 & -5.5638920 & -0.1122370 \\
\hline C & 7.0286770 & -2.9829930 & 0.7820620 \\
\hline C & 8.0023090 & -1.9859060 & 0.1535030 \\
\hline $\mathrm{H}$ & 7.5425680 & -3.9309980 & 0.9768900 \\
\hline $\mathrm{H}$ & 6.6799400 & -2.6238320 & 1.7556720 \\
\hline $\mathrm{H}$ & 8.8506710 & -1.7955750 & 0.8180570 \\
\hline $\mathrm{H}$ & 8.3882070 & -2.3770500 & -0.7931870 \\
\hline $\mathrm{H}$ & 7.5030280 & -1.0354770 & -0.0552340 \\
\hline
\end{tabular}

6 (excited-state/enol) G= -1591.982839 au

$\begin{array}{lrrr}\text { C } & -0.3148740 & 5.5884930 & 0.0048180 \\ \text { C } & 1.0890270 & 5.4802900 & 0.0359940 \\ \text { C } & 1.9441570 & 6.5601260 & 0.0610960 \\ \text { C } & 1.3420720 & 7.8272960 & 0.0541400 \\ \text { C } & -0.0490960 & 7.9638520 & 0.0237350 \\ \text { C } & -0.9005970 & 6.8585350 & -0.0012290 \\ \text { C } & 0.1991960 & 3.4937400 & 0.0049690 \\ \text { H } & -1.9788990 & 6.9709730 & -0.0246730 \\ \text { N } & -0.8487040 & 4.3252650 & -0.0143630 \\ \text { H } & 3.0203280 & 6.4311060 & 0.0845490 \\ \text { O } & 1.4115550 & 4.1495700 & 0.0367650 \\ \text { H } & -0.4803620 & 8.9603900 & 0.0194450 \\ \text { C } & 0.1611020 & 2.0835850 & 0.0080470 \\ \text { C } & -1.1321650 & 1.4205070 & -0.0252470 \\ \text { C } & 1.3110740 & 1.2852730 & 0.0463970 \\ \text { C } & -1.2274340 & 0.0092160 & -0.0449250\end{array}$




\begin{tabular}{|c|c|c|c|}
\hline C & 1.2300230 & -0.1259670 & 0.0177660 \\
\hline$H$ & 2.2749580 & 1.7800360 & 0.0360330 \\
\hline C & -0.0268030 & -0.7468350 & -0.0244690 \\
\hline 0 & -2.2321890 & 2.1542540 & 0.0040390 \\
\hline $\mathrm{H}$ & -1.9809800 & 3.1378620 & 0.0083180 \\
\hline $\mathrm{H}$ & 1.9685310 & 8.7127500 & 0.0726620 \\
\hline C & 2.4584100 & -0.9131530 & -0.0011740 \\
\hline C & 2.4943470 & -2.2236740 & -0.5220880 \\
\hline C & 3.6750370 & -0.4074360 & 0.5033380 \\
\hline C & 3.6489990 & -2.9758510 & -0.5450390 \\
\hline $\mathrm{H}$ & 1.5937660 & -2.6502410 & -0.9512090 \\
\hline C & 4.8386180 & -1.1459880 & 0.4985990 \\
\hline $\mathrm{H}$ & 3.7001140 & 0.5849330 & 0.9409400 \\
\hline C & 4.8690150 & -2.4623230 & -0.0314890 \\
\hline $\mathrm{H}$ & 3.6073220 & -3.9762170 & -0.9580270 \\
\hline $\mathrm{H}$ & 5.7385220 & -0.6961110 & 0.8993070 \\
\hline C & -2.5226780 & -0.6683630 & -0.0187910 \\
\hline C & -3.6815560 & -0.1652790 & -0.6485050 \\
\hline C & -2.6564630 & -1.9182220 & 0.6213080 \\
\hline C & -4.8776160 & -0.8534770 & -0.6395590 \\
\hline $\mathrm{H}$ & -3.6338710 & 0.7825420 & -1.1691830 \\
\hline C & -3.8478770 & -2.6150960 & 0.6481330 \\
\hline $\mathrm{H}$ & -1.8025070 & -2.3377230 & 1.1444980 \\
\hline C & -5.0068920 & -2.1047680 & 0.0137010 \\
\hline $\mathrm{H}$ & -5.7289540 & -0.4084410 & -1.1405050 \\
\hline $\mathrm{H}$ & -3.8782980 & -3.5685040 & 1.1619490 \\
\hline $\mathrm{H}$ & -0.0960520 & -1.8265190 & -0.0453390 \\
\hline$N$ & 6.0174940 & -3.1992850 & -0.0482010 \\
\hline$N$ & -6.1949820 & -2.7880990 & 0.0300180 \\
\hline C & 6.0863700 & -4.5007710 & -0.7004360 \\
\hline C & 5.6542730 & -5.6424260 & 0.2177590 \\
\hline $\mathrm{H}$ & 7.1228290 & -4.6473650 & -1.0204390 \\
\hline $\mathrm{H}$ & 5.4857680 & -4.4804030 & -1.6138950 \\
\hline $\mathrm{H}$ & 5.7300890 & -6.6024440 & -0.3005100 \\
\hline $\mathrm{H}$ & 6.2916030 & -5.6794550 & 1.1064040 \\
\hline $\mathrm{H}$ & 4.6210580 & -5.5056080 & 0.5491620 \\
\hline C & 7.2439680 & -2.7237550 & 0.5794620 \\
\hline C & 8.0741510 & -1.8376230 & -0.3471760 \\
\hline $\mathrm{H}$ & 7.8204560 & -3.6072720 & 0.8713170 \\
\hline $\mathrm{H}$ & 6.9963110 & -2.2012160 & 1.5074890 \\
\hline $\mathrm{H}$ & 8.9898600 & -1.5101100 & 0.1529420 \\
\hline $\mathrm{H}$ & 8.3531890 & -2.3866200 & -1.2515810 \\
\hline $\mathrm{H}$ & 7.5073700 & -0.9530680 & -0.6509470 \\
\hline C & -6.3651920 & -4.0060460 & 0.8079040 \\
\hline C & -5.9295370 & -5.2588600 & 0.0491570 \\
\hline $\mathrm{H}$ & -7.4253880 & -4.0784640 & 1.0718270 \\
\hline $\mathrm{H}$ & -5.8226540 & -3.9117110 & 1.7532420 \\
\hline $\mathrm{H}$ & -6.0772330 & -6.1530670 & 0.6616580 \\
\hline $\mathrm{H}$ & -6.5136040 & -5.3702390 & -0.8695190 \\
\hline $\mathrm{H}$ & -4.8735410 & -5.1967700 & -0.2286180 \\
\hline C & -7.3550500 & -2.3239100 & -0.7163650 \\
\hline C & -8.1906940 & -1.3068950 & 0.0597880 \\
\hline $\mathrm{H}$ & -7.9618130 & -3.2038810 & -0.9541930 \\
\hline $\mathrm{H}$ & -7.0278850 & -1.9120550 & -1.6754680 \\
\hline $\mathrm{H}$ & -9.0551150 & -0.9869790 & -0.5292190 \\
\hline $\mathrm{H}$ & -8.5540040 & -1.7456710 & 0.9941270 \\
\hline $\mathrm{H}$ & -7.5944000 & -0.4253400 & 0.3111390 \\
\hline
\end{tabular}

6 (excited-state/keto) G= $-1591.988426 \mathrm{au}$

$\begin{array}{llll}\text { C } & 0.1225840 & 5.6780120 & 0.0140040\end{array}$ 


\begin{tabular}{|c|c|c|c|}
\hline C & -1.2641450 & 5.4678490 & 0.0002200 \\
\hline$C$ & -2.1808880 & 6.4956180 & -0.0018460 \\
\hline C & -1.6530090 & 7.7962870 & 0.0105170 \\
\hline C & -0.2758860 & 8.0193580 & 0.0237090 \\
\hline C & 0.6458330 & 6.9675130 & 0.0257150 \\
\hline C & -0.3032230 & 3.4820070 & -0.0009930 \\
\hline $\mathrm{H}$ & 1.7149130 & 7.1443720 & 0.0359900 \\
\hline $\mathrm{N}$ & 0.6803270 & 4.4221280 & 0.0125500 \\
\hline $\mathrm{H}$ & -3.2467240 & 6.3011710 & -0.0122070 \\
\hline $\mathrm{O}$ & -1.5124700 & 4.1187230 & -0.0100650 \\
\hline $\mathrm{H}$ & 0.0950890 & 9.0390900 & 0.0328320 \\
\hline C & -0.1879700 & 2.0770890 & -0.0157430 \\
\hline C & 1.1695520 & 1.4927330 & 0.0023520 \\
\hline C & -1.3125100 & 1.2655930 & -0.0446440 \\
\hline C & 1.2562470 & 0.0491320 & 0.0125610 \\
\hline C & -1.2022740 & -0.1545040 & -0.0209070 \\
\hline $\mathrm{H}$ & -2.2938500 & 1.7290390 & -0.0303830 \\
\hline C & 0.0686890 & -0.7220840 & 0.0024700 \\
\hline $\mathrm{O}$ & 2.1682220 & 2.2584430 & -0.0165920 \\
\hline $\mathrm{H}$ & -2.3323140 & 8.6416510 & 0.0098360 \\
\hline C & -2.4144180 & -0.9764620 & 0.0041040 \\
\hline C & -2.4297290 & -2.2610610 & 0.5762930 \\
\hline C & -3.6265670 & -0.5269010 & -0.5487760 \\
\hline C & -3.5647050 & -3.0491860 & 0.5915470 \\
\hline $\mathrm{H}$ & -1.5298520 & -2.6443330 & 1.0478900 \\
\hline C & -4.7727410 & -1.3009510 & -0.5451370 \\
\hline $\mathrm{H}$ & -3.6689860 & 0.4488960 & -1.0234220 \\
\hline C & -4.7807650 & -2.5946550 & 0.0264160 \\
\hline $\mathrm{H}$ & -3.5049810 & -4.0313620 & 1.0449960 \\
\hline $\mathrm{H}$ & -5.6740780 & -0.8903940 & -0.9842200 \\
\hline C & 2.5532480 & -0.6168210 & -0.0095340 \\
\hline C & 3.7285670 & -0.0442000 & 0.5271760 \\
\hline C & 2.6954000 & -1.9109360 & -0.5566870 \\
\hline C & 4.9378650 & -0.7111050 & 0.5261470 \\
\hline $\mathrm{H}$ & 3.6770770 & 0.9471370 & 0.9564530 \\
\hline C & 3.8995260 & -2.5869710 & -0.5722520 \\
\hline $\mathrm{H}$ & 1.8384080 & -2.3907990 & -1.0192130 \\
\hline C & 5.0696520 & -2.0085110 & -0.0253570 \\
\hline $\mathrm{H}$ & 5.7993010 & -0.2101240 & 0.9522610 \\
\hline $\mathrm{H}$ & 3.9300650 & -3.5773800 & -1.0110430 \\
\hline $\mathrm{H}$ & 0.1551190 & -1.8024910 & 0.0257960 \\
\hline $\mathrm{N}$ & -5.9133690 & -3.3710610 & 0.0368900 \\
\hline$N$ & 6.2720520 & -2.6722530 & -0.0310070 \\
\hline C & -5.9589110 & -4.6455530 & 0.7362500 \\
\hline C & -5.4489190 & -5.8085080 & -0.1136910 \\
\hline $\mathrm{H}$ & -7.0004300 & -4.8225110 & 1.0246630 \\
\hline $\mathrm{H}$ & -5.3956010 & -4.5675510 & 1.6711320 \\
\hline $\mathrm{H}$ & -5.5062250 & -6.7495220 & 0.4412590 \\
\hline $\mathrm{H}$ & -6.0502900 & -5.9075320 & -1.0226420 \\
\hline $\mathrm{H}$ & -4.4103510 & -5.6440350 & -0.4145010 \\
\hline C & -7.1255880 & -2.9608680 & -0.6542270 \\
\hline C & -8.0221980 & -2.0644970 & 0.1987870 \\
\hline $\mathrm{H}$ & -7.6659390 & -3.8714440 & -0.9341980 \\
\hline $\mathrm{H}$ & -6.8583650 & -2.4667640 & -1.5933110 \\
\hline $\mathrm{H}$ & -8.9241320 & -1.7810430 & -0.3516210 \\
\hline $\mathrm{H}$ & -8.3257780 & -2.5875090 & 1.1109090 \\
\hline $\mathrm{H}$ & -7.4933690 & -1.1537380 & 0.4939010 \\
\hline C & 6.4365580 & -3.9467330 & -0.7114570 \\
\hline C & 6.0495900 & -5.1401340 & 0.1614010 \\
\hline $\mathrm{H}$ & 7.4879980 & -4.0260940 & -1.0075150 \\
\hline $\mathrm{H}$ & 5.8594190 & -3.9393220 & -1.6410660 \\
\hline $\mathrm{H}$ & 6.1889240 & -6.0797420 & -0.3814160 \\
\hline $\mathrm{H}$ & 6.6684200 & -5.1684430 & 1.0635530 \\
\hline
\end{tabular}




$\begin{array}{llll}\text { H } & 5.0036490 & -5.0689480 & 0.4729600 \\ \mathrm{C} & 7.4459440 & -2.1312620 & 0.6355690 \\ \mathrm{C} & 8.2388190 & -1.1674750 & -0.2465370 \\ \mathrm{H} & 8.0771320 & -2.9785550 & 0.9242430 \\ \mathrm{H} & 7.1424560 & -1.6471110 & 1.5685710 \\ \mathrm{H} & 9.1152280 & -0.7861970 & 0.2858370 \\ \mathrm{H} & 8.5809850 & -1.6754780 & -1.1535120 \\ \mathrm{H} & 7.6182750 & -0.3194650 & -0.5494170 \\ \mathrm{H} & 1.6479490 & 4.0966350 & 0.0061400\end{array}$

6 (excited-state/ESIPT TS) G= $-1591.982708 \mathrm{au}, v(\mathrm{imag})=1147 i \mathrm{~cm}^{-1}$

\begin{tabular}{|c|c|c|c|}
\hline C & 0.9091100 & 5.5020760 & 0.0077950 \\
\hline C & -0.4951000 & 5.6134960 & -0.0195740 \\
\hline C & -1.1635580 & 6.8167770 & -0.0423780 \\
\hline C & -0.3644790 & 7.9709530 & -0.0375160 \\
\hline C & 1.0290060 & 7.8833760 & -0.0111020 \\
\hline C & 1.6948970 & 6.6556890 & 0.0122080 \\
\hline C & 0.0318170 & 3.4978630 & 0.0084960 \\
\hline $\mathrm{H}$ & 2.7768770 & 6.5941750 & 0.0325420 \\
\hline $\mathrm{N}$ & 1.2040540 & 4.1640330 & 0.0248980 \\
\hline $\mathrm{H}$ & -2.2462850 & 6.8617030 & -0.0628730 \\
\hline $\mathrm{O}$ & -1.0379940 & 4.3510060 & -0.0197460 \\
\hline $\mathrm{H}$ & 1.6148970 & 8.7972680 & -0.0083580 \\
\hline C & -0.0701090 & 2.0949630 & -0.0012560 \\
\hline C & 1.2014200 & 1.3576130 & 0.0298810 \\
\hline C & -1.2717420 & 1.3957630 & -0.0387140 \\
\hline C & 1.1724410 & -0.0692120 & 0.0354600 \\
\hline C & -1.2940520 & -0.0240710 & -0.0150360 \\
\hline $\mathrm{H}$ & -2.1994600 & 1.9575220 & -0.0255010 \\
\hline C & -0.0856940 & -0.7244920 & 0.0153670 \\
\hline 0 & 2.3075900 & 2.0275500 & 0.0205470 \\
\hline $\mathrm{H}$ & 2.0352620 & 3.2334620 & 0.0219910 \\
\hline $\mathrm{H}$ & -0.8410110 & 8.9451240 & -0.0546100 \\
\hline C & -2.5777010 & -0.7240340 & 0.0050820 \\
\hline C & -2.7123360 & -2.0143610 & 0.5525990 \\
\hline C & -3.7467600 & -0.1463220 & -0.5261730 \\
\hline C & -3.9178260 & -2.6859150 & 0.5698590 \\
\hline $\mathrm{H}$ & -1.8497830 & -2.4929310 & 1.0056010 \\
\hline C & -4.9610370 & -0.8037950 & -0.5247290 \\
\hline $\mathrm{H}$ & -3.6975990 & 0.8372260 & -0.9826250 \\
\hline C & -5.0901720 & -2.1022830 & 0.0267110 \\
\hline $\mathrm{H}$ & -3.9508450 & -3.6775560 & 1.0045190 \\
\hline $\mathrm{H}$ & -5.8218420 & -0.2999280 & -0.9470950 \\
\hline C & 2.4051450 & -0.8495120 & 0.0065120 \\
\hline C & 3.6193470 & -0.4125620 & 0.5817990 \\
\hline C & 2.4276410 & -2.1309940 & -0.5854820 \\
\hline C & 4.7575520 & -1.1929710 & 0.5729450 \\
\hline $\mathrm{H}$ & 3.6590110 & 0.5597980 & 1.0544650 \\
\hline C & 3.5603320 & -2.9194280 & -0.6113610 \\
\hline $\mathrm{H}$ & 1.5334770 & -2.5053200 & -1.0742560 \\
\hline C & 4.7717810 & -2.4772260 & -0.0259280 \\
\hline $\mathrm{H}$ & 5.6553810 & -0.7948330 & 1.0305760 \\
\hline $\mathrm{H}$ & 3.5029620 & -3.8914170 & -1.0867600 \\
\hline $\mathrm{H}$ & -0.1029530 & -1.8071010 & 0.0339830 \\
\hline$N$ & -6.2898290 & -2.7617250 & 0.0374340 \\
\hline$N$ & 5.9022610 & -3.2527740 & -0.0392600 \\
\hline C & -6.4555220 & -4.0389810 & 0.7163490 \\
\hline C & -6.0796570 & -5.2300080 & -0.1636070 \\
\hline $\mathrm{H}$ & -7.5054590 & -4.1126110 & 1.0178820 \\
\hline $\mathrm{H}$ & -5.8735080 & -4.0358730 & 1.6425310 \\
\hline
\end{tabular}




$\begin{array}{llll}\text { H } & -6.2235050 & -6.1702500 & 0.3764320 \\ \text { H } & -6.7024020 & -5.2508160 & -1.0631260 \\ \text { H } & -5.0344960 & -5.1658090 & -0.4793100 \\ \text { C } & -7.4675800 & -2.2182100 & -0.6238230 \\ \text { C } & -8.2551940 & -1.2570390 & 0.2649740 \\ \text { H } & -8.0989340 & -3.0655060 & -0.9106850 \\ \text { H } & -7.1676310 & -1.7335990 & -1.5574770 \\ \text { H } & -9.1347810 & -0.8764120 & -0.2620650 \\ \text { H } & -8.5917690 & -1.7667790 & 1.1729080 \\ \text { H } & -7.6348370 & -0.4081410 & 0.5658980 \\ \text { C } & 5.9539510 & -4.5152190 & -0.7605970 \\ \text { C } & 5.4351520 & -5.6910960 & 0.0660100 \\ H & 6.9981250 & -4.6874770 & -1.0416630 \\ H & 5.3987750 & -4.4203250 & -1.6985300 \\ \text { H } & 5.4967880 & -6.6225800 & -0.5042390 \\ \text { H } & 6.0280340 & -5.8058600 & 0.9786070 \\ \text { H } & 4.3940010 & -5.5297260 & 0.3592840 \\ \text { C } & 7.1114480 & -2.8551030 & 0.6660820 \\ \text { C } & 8.0120750 & -1.9453960 & -0.1682850 \\ \text { H } & 7.6488280 & -3.7712680 & 0.9329810 \\ \text { H } & 6.8389670 & -2.3762740 & 1.6111260 \\ \text { H } & 8.9118690 & -1.6734380 & 0.3912100 \\ \text { H } & 8.3188010 & -2.4527510 & -1.0881300 \\ \text { H } & 7.4852750 & -1.0289290 & -0.4485680\end{array}$


7 (ground-state) G= $-1257.262995 \mathrm{au}$

$\begin{array}{lrrr}\text { C } & -3.3648450 & -0.8753780 & -0.0556210 \\ \text { C } & -3.8042310 & 0.4485530 & -0.0183910 \\ \text { C } & -5.1368420 & 0.8213810 & -0.0411230 \\ \text { C } & -6.0507260 & -0.2273510 & -0.1057240 \\ \text { C } & -5.6340990 & -1.5683600 & -0.1445010 \\ \text { C } & -4.2885800 & -1.9174220 & -0.1202720 \\ \text { C } & -1.6452710 & 0.3907400 & 0.0390100 \\ \text { H } & -3.9628320 & -2.9510800 & -0.1502390 \\ \text { N } & -1.9730820 & -0.8668140 & -0.0164900 \\ \text { H } & -5.4428520 & 1.8604040 & -0.0108450 \\ \text { O } & -2.6968030 & 1.2510570 & 0.0421200 \\ \text { H } & -6.3844110 & -2.3503540 & -0.1949230 \\ \text { C } & -0.3005100 & 0.9388770 & 0.0928210 \\ \text { C } & 0.8035270 & 0.0581750 & 0.0892040 \\ \text { C } & -0.1062400 & 2.3252950 & 0.1396930 \\ \text { C } & 2.1144360 & 0.5862600 & 0.1308810 \\ \text { C } & 1.1750900 & 2.8431470 & 0.1854200 \\ \text { H } & -0.9730540 & 2.9772830 & 0.1432410 \\ \text { C } & 2.2647840 & 1.9738700 & 0.1840800 \\ \text { O } & 0.6549220 & -1.2733110 & 0.0415700 \\ \text { H } & -0.3060790 & -1.4961260 & -0.0047290 \\ \text { H } & -7.1116210 & -0.0017200 & -0.1267990 \\ \text { H } & 3.2685200 & 2.3857720 & 0.2403990 \\ \text { H } & 1.3341460 & 3.9145160 & 0.2296570 \\ \text { C } & 3.2899600 & -0.2970860 & 0.1003240 \\ \text { C } & 3.4343190 & -1.5918710 & 0.5282990 \\ \text { S } & 4.7978600 & 0.2968140 & -0.5438020 \\ \text { C } & 4.7557250 & -2.0982950 & 0.3545060 \\ \text { H } & 2.6204980 & -2.1581790 & 0.9587760 \\ \text { C } & 5.5991480 & -1.1902490 & -0.2116720 \\ \text { H } & 5.0599730 & -3.0980600 & 0.6407550 \\ \text { H } & 6.6447200 & -1.3074600 & -0.4601630\end{array}$

7 (ground-state/keto) G= $-1257.243152 \mathrm{au}$

$\begin{array}{lccc}\text { C } & -0.7682570 & -3.3829460 & 0.0000000 \\ \text { C } & 0.5652990 & -3.7850470 & 0.0000000 \\ \text { C } & 0.9593670 & -5.1073050 & 0.0000000 \\ \text { C } & -0.0735500 & -6.0458170 & 0.0000000 \\ \text { C } & -1.4185580 & -5.6564470 & 0.0000000 \\ \text { C } & -1.7971250 & -4.3148160 & 0.0000000 \\ \text { C } & 0.5489300 & -1.5938280 & 0.0000000 \\ \text { H } & -2.8377100 & -4.0127240 & 0.0000000 \\ \text { N } & -0.7260840 & -1.9937990 & 0.0000000 \\ \text { H } & 2.0052770 & -5.3895090 & 0.0000000 \\ \text { O } & 1.3655870 & -2.6622390 & 0.0000000 \\ \text { H } & -2.1893640 & -6.4193700 & 0.0000000 \\ \text { C } & 1.0107720 & -0.2680690 & 0.0000000 \\ \text { C } & -0.0000000 & 0.7767960 & 0.0000000 \\ \text { C } & 2.4011460 & 0.0055920 & 0.0000000 \\ \text { C } & 0.5105960 & 2.1455860 & 0.0000000 \\ \text { C } & 2.8300280 & 1.3031270 & 0.0000000 \\ \text { H } & 3.1039660 & -0.8221870 & 0.0000000 \\ \text { C } & 1.8752700 & 2.3483610 & 0.0000000 \\ \text { O } & -1.2306870 & 0.5054650 & 0.0000000 \\ \text { H } & 0.1739890 & -7.1015230 & 0.0000000 \\ \text { H } & 2.2530580 & 3.3685270 & 0.0000000 \\ \text { H } & 3.8870860 & 1.5423070 & 0.0000000 \\ \text { C } & -0.4295640 & 3.2727610 & 0.0000000 \\ \text { C } & -1.8033180 & 3.2775840 & 0.0000000\end{array}$




$\begin{array}{llll}\text { S } & 0.1694340 & 4.9179010 & 0.0000000 \\ \text { C } & -2.3671060 & 4.5883810 & 0.0000000 \\ \text { H } & -2.3766020 & 2.3633830 & 0.0000000 \\ \text { C } & -1.4255710 & 5.5710810 & 0.0000000 \\ \text { H } & -3.4331500 & 4.7838030 & 0.0000000 \\ \text { H } & -1.5686940 & 6.6426930 & 0.0000000 \\ \text { H } & -1.4355630 & -1.2409940 & 0.0000000\end{array}$

7 (ground-state/PT TS) G= $-1257.246472 \mathrm{au}, v(\mathrm{imag})=620 i \mathrm{~cm}^{-1}$

$\begin{array}{lrrr}\text { C } & -3.2852090 & -0.8595950 & 0.0275620 \\ \text { C } & -3.7888290 & 0.4413640 & 0.0228420 \\ \text { C } & -5.1373820 & 0.7358460 & 0.0487680 \\ \text { C } & -5.9940750 & -0.3643870 & 0.0809260 \\ \text { C } & -5.5046340 & -1.6776400 & 0.0861250 \\ \text { C } & -4.1398730 & -1.9545720 & 0.0594810 \\ \text { C } & -1.6164980 & 0.5717860 & -0.0264110 \\ \text { H } & -3.7593120 & -2.9691410 & 0.0634450 \\ \text { N } & -1.9031410 & -0.7221310 & -0.0054100 \\ \text { H } & -5.4985950 & 1.7572050 & 0.0443670 \\ \text { O } & -2.7283310 & 1.3236160 & -0.0112680 \\ \text { H } & -6.2084960 & -2.5024600 & 0.1115780 \\ \text { C } & -0.2970500 & 1.0822610 & -0.0584460 \\ \text { C } & 0.7537270 & 0.0906150 & -0.0633390 \\ \text { C } & -0.0409330 & 2.4689480 & -0.0840280 \\ \text { C } & 2.1080690 & 0.6002540 & -0.0853920 \\ \text { C } & 1.2565290 & 2.9122110 & -0.1163240 \\ \text { H } & -0.8765890 & 3.1625780 & -0.0825630 \\ \text { C } & 2.3056220 & 1.9703230 & -0.1200050 \\ \text { O } & 0.4939440 & -1.1615660 & -0.0465940 \\ \text { H } & -0.9626550 & -1.3039510 & -0.0155050 \\ \text { H } & -7.0653220 & -0.1972170 & 0.1025430 \\ \text { H } & 3.3249970 & 2.3472890 & -0.1615520 \\ \text { H } & 1.4834800 & 3.9715750 & -0.1444420 \\ \text { C } & 3.2405340 & -0.3332960 & -0.0614680 \\ \text { C } & 3.2758310 & -1.6824680 & -0.3126310 \\ \text { S } & 4.8432750 & 0.2438280 & 0.3309620 \\ \text { C } & 4.5831040 & -2.2437640 & -0.2064140 \\ \text { H } & 2.3876040 & -2.2423890 & -0.5640960 \\ \text { C } & 5.5289110 & -1.3248460 & 0.1327760 \\ \text { H } & 4.8034060 & -3.2912560 & -0.3761180 \\ \text { H } & 6.5891020 & -1.4732240 & 0.2835880\end{array}$

7 (excited-state/enol) G= $-1257.132294 \mathrm{au}$

$\begin{array}{lrrr}\text { C } & -0.4407470 & -3.4044030 & 0.0000000 \\ \text { C } & 0.9287280 & -3.7213430 & 0.0000000 \\ \text { C } & 1.4199880 & -5.0099800 & 0.0000000 \\ \text { C } & 0.4623870 & -6.0304500 & 0.0000000 \\ \text { C } & -0.9081340 & -5.7420240 & 0.0000000 \\ \text { C } & -1.3859640 & -4.4330540 & 0.0000000 \\ \text { C } & 0.6837100 & -1.5646770 & 0.0000000 \\ \text { H } & -2.4474940 & -4.2124640 & 0.0000000 \\ \text { N } & -0.5629850 & -2.0340360 & 0.0000000 \\ \text { H } & 2.4845850 & -5.2132310 & 0.0000000 \\ \text { O } & 1.6363960 & -2.5477130 & 0.0000000 \\ \text { H } & -1.6183590 & -6.5628170 & 0.0000000 \\ \text { C } & 1.0607800 & -0.2023350 & 0.0000000 \\ \text { C } & 0.0000000 & 0.7893980 & 0.0000000 \\ \text { C } & 2.3882580 & 0.2459690 & 0.0000000 \\ \text { C } & 0.2961360 & 2.1914070 & 0.0000000 \\ \text { C } & 2.6758230 & 1.6239180 & 0.0000000\end{array}$




$\begin{array}{cccc}\text { H } & 3.1913060 & -0.4821390 & 0.0000000 \\ \mathrm{C} & 1.6832840 & 2.5827500 & 0.0000000 \\ \mathrm{O} & -1.2479370 & 0.3977060 & 0.0000000 \\ \mathrm{H} & -1.2855470 & -0.6347040 & 0.0000000 \\ \mathrm{H} & 0.7908050 & -7.0642520 & 0.0000000 \\ \mathrm{H} & 1.9476530 & 3.6328000 & 0.0000000 \\ \mathrm{H} & 3.7153570 & 1.9365490 & 0.0000000 \\ \mathrm{C} & -0.7422820 & 3.1631890 & 0.0000000 \\ \mathrm{C} & -2.1452340 & 3.0513750 & 0.0000000 \\ \mathrm{~S} & -0.2941280 & 4.8725750 & 0.0000000 \\ \mathrm{C} & -2.7995760 & 4.2896300 & 0.0000000 \\ \mathrm{H} & -2.6528590 & 2.0997080 & 0.0000000 \\ \mathrm{C} & -1.9317100 & 5.3638570 & 0.0000000 \\ \mathrm{H} & -3.8769530 & 4.4022870 & 0.0000000 \\ \mathrm{H} & -2.1819030 & 6.4160100 & 0.0000000\end{array}$

7 (excited-state/keto) G= -1257.143472 au

$\begin{array}{lccc}\text { C } & -0.8752620 & -3.4136480 & 0.0000000 \\ \text { C } & 0.4661790 & -3.8163900 & 0.0000000 \\ \text { C } & 0.8565080 & -5.1378720 & 0.0000000 \\ \text { C } & -0.1766610 & -6.0844960 & 0.0000000 \\ \text { C } & -1.5184970 & -5.6968320 & 0.0000000 \\ \text { C } & -1.9010590 & -4.3530320 & 0.0000000 \\ \text { C } & 0.4496430 & -1.6127220 & 0.0000000 \\ \text { H } & -2.9429090 & -4.0551780 & 0.0000000 \\ \text { N } & -0.8409710 & -2.0367660 & 0.0000000 \\ \text { H } & 1.9030220 & -5.4181410 & 0.0000000 \\ \text { O } & 1.2671560 & -2.7023780 & 0.0000000 \\ \text { H } & -2.2895730 & -6.4600240 & 0.0000000 \\ \text { C } & 0.9634610 & -0.2991740 & 0.0000000 \\ \text { C } & 0.0000000 & 0.8245440 & 0.0000000 \\ \text { C } & 2.3290290 & -0.0568870 & 0.0000000 \\ \text { C } & 0.5474900 & 2.1681880 & 0.0000000 \\ \text { C } & 2.8250190 & 1.2722720 & 0.0000000 \\ \text { H } & 3.0226490 & -0.8915440 & 0.0000000 \\ \text { C } & 1.9704170 & 2.3476580 & 0.0000000 \\ \text { O } & -1.2281000 & 0.5762060 & 0.0000000 \\ \text { H } & 0.0744880 & -7.1393980 & 0.0000000 \\ \text { H } & 2.3821590 & 3.3515490 & 0.0000000 \\ \text { H } & 3.8981860 & 1.4310170 & 0.0000000 \\ \text { C } & -0.3307420 & 3.2968360 & 0.0000000 \\ \text { C } & -1.7243330 & 3.3692210 & 0.0000000 \\ \text { S } & 0.3380810 & 4.9272400 & 0.0000000 \\ \text { C } & -2.2171710 & 4.6907410 & 0.0000000 \\ \text { H } & -2.3377320 & 2.4815610 & 0.0000000 \\ \text { C } & -1.2239480 & 5.6379420 & 0.0000000 \\ \text { H } & -3.2717430 & 4.9401850 & 0.0000000 \\ \text { H } & -1.3286080 & 6.7141930 & 0.0000000 \\ \text { H } & -1.5853240 & -1.3414170 & 0.0000000\end{array}$

7 (excited-state/ESIPT TS) G= $-1257.133651 \mathrm{au}, v(\mathrm{imag})=1119 i \mathrm{~cm}^{-1}$

$\begin{array}{lrrr}\text { C } & -0.2683460 & -3.3680250 & -0.0000000 \\ \text { C } & 1.1072110 & -3.6631900 & -0.0000000 \\ \text { C } & 1.6133780 & -4.9448530 & -0.0000000 \\ \text { C } & 0.6689820 & -5.9792440 & -0.0000000 \\ \text { C } & -0.7036410 & -5.7093370 & -0.0000000 \\ \text { C } & -1.2008590 & -4.4061390 & -0.0000000 \\ \text { C } & 0.8578750 & -1.5034110 & -0.0000000\end{array}$




$\begin{array}{cccc}\mathrm{H} & -2.2651940 & -4.2009160 & -0.0000000 \\ \mathrm{~N} & -0.3892210 & -1.9995780 & -0.0000000 \\ \mathrm{H} & 2.6806080 & -5.1331790 & -0.0000000 \\ \mathrm{O} & 1.8083040 & -2.4815560 & -0.0000000 \\ \mathrm{H} & -1.4035490 & -6.5388310 & -0.0000000 \\ \mathrm{C} & 1.1565460 & -0.1278470 & -0.0000000 \\ \mathrm{C} & 0.0000000 & 0.7770400 & 0.0000000 \\ \mathrm{C} & 2.4411230 & 0.4137540 & 0.0000000 \\ \mathrm{C} & 0.2046880 & 2.1988860 & 0.0000000 \\ \mathrm{C} & 2.6219270 & 1.8139430 & 0.0000000 \\ \mathrm{H} & 3.2990500 & -0.2496560 & -0.0000000 \\ \mathrm{C} & 1.5563320 & 2.6890700 & 0.0000000 \\ \mathrm{O} & -1.1863000 & 0.2718970 & 0.0000000 \\ \mathrm{H} & -1.0964390 & -0.9038690 & -0.0000000 \\ \mathrm{H} & 1.0117630 & -7.0082240 & -0.0000000 \\ \mathrm{H} & 1.7406300 & 3.7568060 & 0.0000000 \\ \mathrm{H} & 3.6336170 & 2.2071110 & 0.0000000 \\ \mathrm{C} & -0.9049540 & 3.0947450 & 0.0000000 \\ \mathrm{C} & -2.2877040 & 2.8645630 & 0.0000000 \\ \mathrm{~S} & -0.6016700 & 4.8315040 & 0.0000000 \\ \mathrm{C} & -3.0483210 & 4.0456460 & 0.0000000 \\ \mathrm{H} & -2.7072960 & 1.8706640 & 0.0000000 \\ \mathrm{C} & -2.2766470 & 5.1862260 & 0.0000000 \\ \mathrm{H} & -4.1314660 & 4.0650570 & 0.0000000 \\ \mathrm{H} & -2.6120320 & 6.2143300 & 0.0000000\end{array}$


8 (ground-state) G= $-952.520006 \mathrm{au}$

\begin{tabular}{lrrr} 
C & -3.6570420 & 0.1313210 & 0.0214920 \\
C & -3.0511750 & -1.1248910 & 0.0725150 \\
C & -3.7498260 & -2.3180810 & 0.1331530 \\
C & -5.1371990 & -2.1990780 & 0.1405570 \\
C & -5.7714860 & -0.9465300 & 0.0895330 \\
C & -5.0470420 & 0.2383440 & 0.0292750 \\
C & -1.5408010 & 0.4161010 & -0.0103560 \\
H & -5.5324760 & 1.2069860 & -0.0100240 \\
N & -2.6509200 & 1.0927130 & -0.0308050 \\
H & -3.2467050 & -3.2769510 & 0.1724130 \\
O & -1.6955650 & -0.9318560 & 0.0511190 \\
H & -6.8556070 & -0.9061920 & 0.0976660 \\
C & -0.1960400 & 0.9637290 & -0.0488690 \\
C & -0.0213700 & 2.3636040 & -0.1057990 \\
C & 0.9213290 & 0.1212750 & -0.0221520 \\
C & 1.2809080 & 2.8781930 & -0.1333690 \\
C & 2.2135010 & 0.6327880 & -0.0476170 \\
H & 0.7597760 & -0.9503580 & 0.0446630 \\
C & 2.3711190 & 2.0292780 & -0.1024460 \\
O & -1.0390050 & 3.2338990 & -0.1357920 \\
H & -1.8927790 & 2.7416120 & -0.1101440 \\
H & -5.7431530 & -3.0976780 & 0.1870410 \\
H & 3.3709990 & 2.4514030 & -0.1462680 \\
H & 1.4029200 & 3.9543300 & -0.1873500 \\
C & 3.3891510 & -0.2667010 & -0.0154550 \\
C & 4.5645740 & 0.0902700 & 0.6524290 \\
C & 3.3760190 & -1.5125000 & -0.6502770 \\
C & 5.6405510 & -0.7907210 & 0.6527820 \\
H & 4.6363700 & 1.0314970 & 1.1881920 \\
C & 4.5057250 & -2.3205830 & -0.5852430 \\
H & 2.5065380 & -1.8417770 & -1.2103940 \\
N & 5.6307880 & -1.9825620 & 0.0502820 \\
H & 6.5583030 & -0.5250950 & 1.1728110 \\
H & 4.5079170 & -3.2900820 & -1.0784810 \\
& & & \\
\hline
\end{tabular}

8 (ground-state/keto) G= -952.500185 au

$\begin{array}{lrrr}\text { C } & -3.6842290 & 0.1516100 & 0.0225130 \\ \text { C } & -3.1036250 & -1.1140520 & 0.0679070 \\ \text { C } & -3.8344180 & -2.2829030 & 0.1265260 \\ \text { C } & -5.2212990 & -2.1280890 & 0.1383800 \\ \text { C } & -5.8190380 & -0.8624610 & 0.0933280 \\ \text { C } & -5.0636410 & 0.3074580 & 0.0342510 \\ \text { C } & -1.4735720 & 0.3450360 & -0.0145670 \\ \text { H } & -5.5252870 & 1.2871130 & -0.0005390 \\ \text { N } & -2.6150010 & 1.0380510 & -0.0288690 \\ \text { H } & -3.3545520 & -3.2535010 & 0.1611660 \\ \text { O } & -1.7312830 & -0.9721060 & 0.0436370 \\ \text { H } & -6.9010220 & -0.7888760 & 0.1047410 \\ \text { C } & -0.1817670 & 0.8930820 & -0.0552000 \\ \text { C } & -0.0893680 & 2.3437800 & -0.1096090 \\ \text { C } & 0.9647800 & 0.0637580 & -0.0318430 \\ \text { C } & 1.2611460 & 2.8567750 & -0.1360210 \\ \text { C } & 2.2283770 & 0.6026510 & -0.0556980 \\ \text { H } & 0.8264910 & -1.0124420 & 0.0344660 \\ \text { C } & 2.3443010 & 2.0296500 & -0.1062340 \\ \text { O } & -1.0970020 & 3.0975490 & -0.1330790 \\ \text { H } & -5.8492760 & -3.0109160 & 0.1836680 \\ \text { H } & 3.3397930 & 2.4649960 & -0.1492530 \\ \text { H } & 1.3736610 & 3.9345310 & -0.1899260\end{array}$




$\begin{array}{lrrr}\text { C } & 3.4323260 & -0.2518390 & -0.0191880 \\ \text { C } & 4.6104820 & 0.1628460 & 0.6128600 \\ \text { C } & 3.4542080 & -1.5207610 & -0.6107010 \\ \text { C } & 5.7136140 & -0.6834250 & 0.6255500 \\ \text { H } & 4.6649180 & 1.1240100 & 1.1140480 \\ \text { C } & 4.6084970 & -2.2905750 & -0.5352630 \\ \text { H } & 2.5893770 & -1.8963190 & -1.1484160 \\ \text { N } & 5.7341110 & -1.8968930 & 0.0678060 \\ \text { H } & 6.6307450 & -0.3689300 & 1.1191820 \\ \text { H } & 4.6327120 & -3.2763680 & -0.9950250 \\ \text { H } & -2.5157010 & 2.0698030 & -0.0730860\end{array}$

8 (ground-state/PT TS) G= $-952.501907 \mathrm{au}, v(\mathrm{imag})=591 i \mathrm{~cm}^{-1}$

\begin{tabular}{|c|c|c|c|}
\hline C & -3.6724280 & 0.1618280 & 0.0201000 \\
\hline C & -3.1396310 & -1.1264260 & 0.0716910 \\
\hline C & -3.9126290 & -2.2686050 & 0.1329920 \\
\hline C & -5.2920480 & -2.0628450 & 0.1410190 \\
\hline C & -5.8438040 & -0.7754800 & 0.0899610 \\
\hline C & -5.0469050 & 0.3651300 & 0.0283870 \\
\hline C & -1.4767520 & 0.2745740 & -0.0123160 \\
\hline $\mathrm{H}$ & -5.4717960 & 1.3611480 & -0.0109260 \\
\hline $\mathrm{N}$ & -2.5765100 & 1.0146310 & -0.0325180 \\
\hline $\mathrm{H}$ & -3.4692750 & -3.2562800 & 0.1722990 \\
\hline 0 & -1.7623440 & -1.0344160 & 0.0501950 \\
\hline $\mathrm{H}$ & -6.9225810 & -0.6635780 & 0.0987080 \\
\hline C & -0.1741670 & 0.8243510 & -0.0542630 \\
\hline C & -0.1110490 & 2.2679450 & -0.1135030 \\
\hline C & 0.9830750 & 0.0213620 & -0.0283370 \\
\hline C & 1.2123260 & 2.8176700 & -0.1437330 \\
\hline C & 2.2377860 & 0.5914990 & -0.0550310 \\
\hline $\mathrm{H}$ & 0.8680270 & -1.0574160 & 0.0414970 \\
\hline C & 2.3193890 & 2.0138710 & -0.1116710 \\
\hline 0 & -1.1527110 & 3.0043920 & -0.1380020 \\
\hline $\mathrm{H}$ & -2.3069960 & 2.0848320 & -0.0838890 \\
\hline $\mathrm{H}$ & -5.9522100 & -2.9218640 & 0.1880470 \\
\hline $\mathrm{H}$ & 3.3030880 & 2.4745530 & -0.1573410 \\
\hline $\mathrm{H}$ & 1.2995220 & 3.8973610 & -0.2015060 \\
\hline C & 3.4611010 & -0.2361600 & -0.0172730 \\
\hline C & 4.6267830 & 0.2033540 & 0.6204780 \\
\hline C & 3.5130120 & -1.5014930 & -0.6136450 \\
\hline C & 5.7492660 & -0.6170800 & 0.6326960 \\
\hline $\mathrm{H}$ & 4.6566040 & 1.1634400 & 1.1258400 \\
\hline C & 4.6849030 & -2.2446660 & -0.5383100 \\
\hline $\mathrm{H}$ & 2.6581770 & -1.8948030 & -1.1546730 \\
\hline N & 5.7990450 & -1.8272810 & 0.0697150 \\
\hline $\mathrm{H}$ & 6.6574770 & -0.2838690 & 1.1304200 \\
\hline $\mathrm{H}$ & 4.7332900 & -3.2277470 & -1.0018410 \\
\hline
\end{tabular}

8 (excited-state/enol) $\mathrm{G}=-952.379737 \mathrm{au}$

$\begin{array}{lrrr}\text { C } & -3.6602390 & 0.1767640 & 0.0163940 \\ \text { C } & -3.1029270 & -1.1282910 & 0.0676790 \\ \text { C } & -3.8537840 & -2.2829930 & 0.1246090 \\ \text { C } & -5.2436580 & -2.1093450 & 0.1301700 \\ \text { C } & -5.8248980 & -0.8292410 & 0.0802010 \\ \text { C } & -5.0599290 & 0.3276980 & 0.0229210 \\ \text { C } & -1.5120360 & 0.3490030 & -0.0109120 \\ \text { H } & -5.5090640 & 1.3134070 & -0.0152680 \\ \text { N } & -2.6500180 & 1.0784270 & -0.0318080 \\ \text { H } & -3.3904850 & -3.2618500 & 0.1628510 \\ \text { O } & -1.7458950 & -1.0104030 & 0.0499830\end{array}$




$\begin{array}{llll}\text { H } & -6.9072500 & -0.7474450 & 0.0868930 \\ \mathrm{C} & -0.2076210 & 0.8470390 & -0.0429790 \\ \mathrm{C} & -0.0179900 & 2.3033140 & -0.1055790 \\ \mathrm{C} & 0.9493420 & 0.0361000 & -0.0069390 \\ \mathrm{C} & 1.2715300 & 2.8494060 & -0.1286820 \\ \mathrm{C} & 2.2277950 & 0.6043290 & -0.0278910 \\ \mathrm{H} & 0.8250950 & -1.0366120 & 0.0834740 \\ \mathrm{C} & 2.3827740 & 2.0173050 & -0.0845000 \\ \mathrm{O} & -1.0516420 & 3.1110130 & -0.1418520 \\ \mathrm{H} & -1.9101460 & 2.5669470 & -0.1124630 \\ \mathrm{H} & -5.8853760 & -2.9828500 & 0.1738840 \\ \mathrm{H} & 3.3765170 & 2.4461200 & -0.1449140 \\ \mathrm{H} & 1.3631430 & 3.9277470 & -0.1905690 \\ \mathrm{C} & 3.4228970 & -0.2547420 & -0.0068750 \\ \mathrm{C} & 4.6273080 & 0.1744700 & 0.5671930 \\ \mathrm{C} & 3.4072560 & -1.5441290 & -0.5560810 \\ \mathrm{C} & 5.7224700 & -0.6810660 & 0.5659550 \\ \mathrm{H} & 4.7051440 & 1.1481670 & 1.0388340 \\ \mathrm{C} & 4.5626220 & -2.3152370 & -0.5100380 \\ \mathrm{H} & 2.5177350 & -1.9311580 & -1.0412490 \\ \mathrm{~N} & 5.7106560 & -1.9089010 & 0.0389570 \\ \mathrm{H} & 6.6593120 & -0.3629440 & 1.0171970 \\ \mathrm{H} & 4.5657370 & -3.3133980 & -0.9416320 \\ & & & \end{array}$

8 (excited-state/keto) G $=-952.389068 \mathrm{au}$

$\begin{array}{lrrr}\text { C } & -3.7090930 & 0.1692300 & 0.0232400 \\ \text { C } & -3.0993320 & -1.0942300 & 0.0524650 \\ \text { C } & -3.8105850 & -2.2742720 & 0.0983160 \\ \text { C } & -5.2056630 & -2.1494480 & 0.1150180 \\ \text { C } & -5.8265810 & -0.8968360 & 0.0859910 \\ \text { C } & -5.0966070 & 0.2919830 & 0.0394090 \\ \text { C } & -1.4892870 & 0.4039930 & -0.0178740 \\ \text { H } & -5.5807160 & 1.2610380 & 0.0171650 \\ \text { N } & -2.6732050 & 1.0705480 & -0.0179930 \\ \text { H } & -3.3109370 & -3.2352730 & 0.1199350 \\ \text { O } & -1.7383630 & -0.9338030 & 0.0272130 \\ \text { H } & -6.9101900 & -0.8448850 & 0.1000780 \\ \mathrm{C} & -0.1727860 & 0.9231210 & -0.0450510 \\ \mathrm{C} & -0.0053840 & 2.3938010 & -0.1052180 \\ \mathrm{C} & 0.9414790 & 0.0994960 & 0.0006850 \\ \mathrm{C} & 1.3368250 & 2.8849170 & -0.1148190 \\ \mathrm{C} & 2.2655920 & 0.6388940 & -0.0154040 \\ \mathrm{H} & 0.8020940 & -0.9725400 & 0.0918330 \\ \mathrm{C} & 2.4333760 & 2.0337330 & -0.0665530 \\ \mathrm{O} & -1.0108830 & 3.1428740 & -0.1428540 \\ \mathrm{H} & -5.8150420 & -3.0456930 & 0.1510500 \\ \mathrm{H} & 3.4333540 & 2.4512090 & -0.1191930 \\ \mathrm{H} & 1.4596820 & 3.9607540 & -0.1782060 \\ \mathrm{C} & 3.4236200 & -0.2566130 & 0.0009440 \\ \mathrm{C} & 4.6703580 & 0.1500740 & 0.5053660 \\ \mathrm{C} & 3.3467990 & -1.5721930 & -0.4850560 \\ \mathrm{C} & 5.7345910 & -0.7401370 & 0.4942370 \\ \mathrm{H} & 4.8027680 & 1.1376250 & 0.9335540 \\ \mathrm{C} & 4.4777470 & -2.3792050 & -0.4531880 \\ \mathrm{H} & 2.4286880 & -1.9556040 & -0.9166470 \\ \mathrm{~N} & 5.6624990 & -1.9917510 & 0.0260560 \\ \mathrm{H} & 6.6998320 & -0.4338960 & 0.8909750 \\ \mathrm{H} & 4.4269200 & -3.3954340 & -0.8378590 \\ \mathrm{H} & -2.6579730 & 2.0907120 & -0.0590510\end{array}$


8 (excited-state/ESIPT TS) G= $-952.380249 \mathrm{au}, v(\mathrm{imag})=1217 i \mathrm{~cm}^{-1}$

$\begin{array}{lrrr}\text { C } & -3.1733930 & -1.1280140 & 0.0690890 \\ \text { C } & -3.9812260 & -2.2431880 & 0.1250330 \\ \text { C } & -5.3614150 & -2.0033010 & 0.1249000 \\ \text { C } & -5.8754030 & -0.7004450 & 0.0707050 \\ \text { C } & -5.0501770 & 0.4198010 & 0.0145070 \\ \text { C } & -1.4885180 & 0.2439550 & -0.0060900 \\ \text { H } & -5.4512260 & 1.4257560 & -0.0270020 \\ \text { N } & -2.5892540 & 1.0277950 & -0.0316630 \\ \text { H } & -3.5652280 & -3.2429360 & 0.1663940 \\ \text { O } & -1.8039440 & -1.0858360 & 0.0563140 \\ \text { H } & -6.9516810 & -0.5606000 & 0.0727770 \\ \text { C } & -0.1800680 & 0.7473920 & -0.0406810 \\ \text { C } & -0.0579940 & 2.2222360 & -0.1080020 \\ \mathrm{C} & 0.9872610 & -0.0202930 & -0.0024940 \\ \mathrm{C} & 1.2291430 & 2.8032720 & -0.1326220 \\ \mathrm{C} & 2.2602810 & 0.5950720 & -0.0259350 \\ \mathrm{H} & 0.9035630 & -1.0970520 & 0.0926550 \\ \mathrm{C} & 2.3662010 & 2.0074020 & -0.0858940 \\ \mathrm{O} & -1.1214730 & 2.9473700 & -0.1418380 \\ \mathrm{H} & -2.0734750 & 2.2129270 & -0.0978960 \\ \mathrm{H} & -6.0443010 & -2.8448230 & 0.1676310 \\ \mathrm{H} & 3.3450890 & 2.4686630 & -0.1494310 \\ \mathrm{H} & 1.2885880 & 3.8836870 & -0.1995620 \\ \mathrm{C} & 3.4753060 & -0.2289880 & -0.0038900 \\ \mathrm{C} & 4.6800070 & 0.2468940 & 0.5351340 \\ \mathrm{C} & 3.4857620 & -1.5342740 & -0.5177640 \\ \mathrm{C} & 5.7975550 & -0.5779790 & 0.5336220 \\ \mathrm{H} & 4.7405220 & 1.2333270 & 0.9816660 \\ \mathrm{C} & 4.6632090 & -2.2714170 & -0.4759350 \\ \mathrm{H} & 2.5998100 & -1.9607090 & -0.9752390 \\ \mathrm{~N} & 5.8098110 & -1.8198240 & 0.0389460 \\ \mathrm{H} & 6.7336640 & -0.2228840 & 0.9578470 \\ \mathrm{H} & 4.6852010 & -3.2802370 & -0.8813570\end{array}$


Below are given the Cartesian $(\AA)$ coordinates for the second series of structures as obtained at PCM(DCM)-M06-2X/6-31G(d) level of theory. Except when noted, all structures are true minima, with no imaginary frequency. For each structure, we provide the free energy as obtained with the same level of theory.

\section{0 (ground-state) G=-952.522041au}

$\begin{array}{lrrr}\text { C } & -3.2730670 & -0.9156800 & 0.0883480 \\ \text { C } & -3.7664390 & 0.3864560 & -0.0046020 \\ \text { C } & -5.1138420 & 0.7033810 & -0.0186930 \\ \text { C } & -5.9836240 & -0.3809510 & 0.0677850 \\ \text { C } & -5.5121040 & -1.7014090 & 0.1624050 \\ \text { C } & -4.1528770 & -1.9939370 & 0.1745220 \\ \text { C } & -1.6067280 & 0.4181390 & -0.0231600 \\ \text { H } & -3.7854820 & -3.0114230 & 0.2473830 \\ \text { N } & -1.8819880 & -0.8494200 & 0.0729620 \\ \text { H } & -5.4633280 & 1.7263820 & -0.0921360 \\ \text { O } & -2.6914260 & 1.2324190 & -0.0753070 \\ \text { H } & -6.2300680 & -2.5120910 & 0.2275790 \\ \text { C } & -0.2829460 & 1.0160820 & -0.0768300 \\ \text { C } & 0.8537490 & 0.1786990 & -0.0294170 \\ \text { C } & -0.1353590 & 2.4059900 & -0.1686400 \\ \text { C } & 2.1392170 & 0.7571930 & -0.0714070 \\ \text { C } & 1.1261920 & 2.9730930 & -0.2093880 \\ \text { H } & -1.0247780 & 3.0259020 & -0.2079880 \\ \text { C } & 2.2474940 & 2.1450550 & -0.1576670 \\ \text { O } & 0.7592140 & -1.1562120 & 0.0744770 \\ \text { H } & -0.1937700 & -1.4159310 & 0.1086980 \\ \text { H } & -7.0532060 & -0.2001920 & 0.0618470 \\ \text { H } & 3.2399160 & 2.5840940 & -0.2057570 \\ \text { H } & 1.2433950 & 4.0478790 & -0.2860340 \\ \text { C } & 3.3660090 & -0.0757530 & -0.0120260 \\ \text { C } & 4.4611500 & 0.3346690 & 0.7541900 \\ \text { C } & 3.5062850 & -1.2647740 & -0.7351820 \\ \text { C } & 5.6173530 & -0.4384610 & 0.7594540 \\ \text { H } & 4.4107300 & 1.2372250 & 1.3549970 \\ \text { C } & 4.7070250 & -1.9617720 & -0.6616530 \\ \text { H } & 2.6962490 & -1.6394330 & -1.3489140 \\ \text { N } & 5.7576540 & -1.5726250 & 0.0680800 \\ \text { H } & 6.4730870 & -0.1292650 & 1.3554680 \\ \text { H } & 4.8303720 & -2.8846130 & -1.2240310\end{array}$

10 (excited-state/enol) $\mathrm{G}=-952.384236$ au

$\begin{array}{lrrr}\text { C } & -3.2108890 & -0.9169580 & 0.0691830 \\ \text { C } & -3.7634710 & 0.3915590 & 0.0073740 \\ \text { C } & -5.1168770 & 0.6550480 & 0.0139380 \\ \text { C } & -5.9550370 & -0.4640800 & 0.0869400 \\ \text { C } & -5.4371280 & -1.7730590 & 0.1494690 \\ \text { C } & -4.0739730 & -2.0285600 & 0.1424280 \\ \text { C } & -1.5940020 & 0.5073610 & -0.0321340 \\ \text { H } & -3.6760370 & -3.0355420 & 0.1905690 \\ \text { N } & -1.8624150 & -0.8167960 & 0.0423500 \\ \text { H } & -5.5016190 & 1.6667940 & -0.0342320 \\ \text { O } & -2.7363300 & 1.2813480 & -0.0558240 \\ \text { H } & -6.1312540 & -2.6054710 & 0.2048830 \\ \text { C } & -0.3338940 & 1.1023200 & -0.0793320\end{array}$




$\begin{array}{lrrr}\text { C } & 0.8504260 & 0.2266470 & -0.0517760 \\ \text { C } & -0.1314770 & 2.5041840 & -0.1424160 \\ \text { C } & 2.1592890 & 0.7680700 & -0.0948820 \\ \text { C } & 1.1595420 & 3.0072900 & -0.1797750 \\ \text { H } & -0.9921560 & 3.1614940 & -0.1598230 \\ \text { C } & 2.2896060 & 2.1659040 & -0.1549930 \\ \text { O } & 0.6917640 & -1.0691050 & 0.0532540 \\ \text { H } & -0.3023810 & -1.2995470 & 0.0759310 \\ \text { H } & -7.0299360 & -0.3189430 & 0.0957740 \\ \text { H } & 3.2796630 & 2.6021920 & -0.2227320 \\ \text { H } & 1.3085450 & 4.0807690 & -0.2389620 \\ \text { C } & 3.3486450 & -0.0965350 & -0.0257690 \\ \text { C } & 4.5013740 & 0.3504780 & 0.6394930 \\ \text { C } & 3.4279470 & -1.3591180 & -0.6344080 \\ \text { C } & 5.6314710 & -0.4559610 & 0.6702520 \\ \text { H } & 4.5084400 & 1.3059420 & 1.1535690 \\ \text { C } & 4.6111280 & -2.0815470 & -0.5458010 \\ \text { H } & 2.5900450 & -1.7677130 & -1.1845340 \\ \text { N } & 5.7086970 & -1.6592840 & 0.0921940 \\ \text { H } & 6.5227660 & -0.1163820 & 1.1933800 \\ \text { H } & 4.6804010 & -3.0572420 & -1.0218110\end{array}$

10 (excited-state/keto) $\mathrm{G}=-952.398486 \mathrm{au}$

$\begin{array}{lrrr}\text { C } & -3.3262240 & -0.9303310 & 0.0756500 \\ \text { C } & -3.7786470 & 0.3922820 & -0.0150820 \\ \text { C } & -5.1147340 & 0.7330970 & -0.0497570 \\ \text { C } & -6.0183400 & -0.3332350 & 0.0121260 \\ \text { C } & -5.5800750 & -1.6597240 & 0.1030520 \\ \text { C } & -4.2253730 & -1.9912760 & 0.1369880 \\ \text { C } & -1.5774990 & 0.4577900 & 0.0006820 \\ \text { H } & -3.8871910 & -3.0181670 & 0.2070340 \\ \text { N } & -1.9523710 & -0.8442450 & 0.0835380 \\ \text { H } & -5.4350450 & 1.7655650 & -0.1204310 \\ \text { O } & -2.6950050 & 1.2303140 & -0.0590830 \\ \text { H } & -6.3150880 & -2.4562300 & 0.1486660 \\ \mathrm{C} & -0.2924500 & 1.0382100 & -0.0382690 \\ \mathrm{C} & 0.8883780 & 0.1362170 & -0.0016280 \\ \mathrm{C} & -0.1206010 & 2.4184600 & -0.0898110 \\ \mathrm{C} & 2.2070850 & 0.7397730 & -0.0586920 \\ \mathrm{C} & 1.1831900 & 2.9616750 & -0.1442050 \\ \mathrm{H} & -0.9845110 & 3.0738570 & -0.0912160 \\ \mathrm{C} & 2.3106550 & 2.1538070 & -0.1263050 \\ \mathrm{O} & 0.6949880 & -1.0934700 & 0.1133000 \\ \mathrm{H} & -7.0821640 & -0.1245300 & -0.0110520 \\ \mathrm{H} & 3.2881140 & 2.6127860 & -0.2191390 \\ \mathrm{H} & 1.2970720 & 4.0386560 & -0.2131720 \\ \mathrm{C} & 3.4051430 & -0.0883410 & -0.0206640 \\ \mathrm{C} & 4.6267990 & 0.4380870 & 0.4525920 \\ \mathrm{C} & 3.4522290 & -1.4262630 & -0.4677460 \\ \mathrm{C} & 5.7657300 & -0.3526450 & 0.4610110 \\ \mathrm{H} & 4.6826360 & 1.4459490 & 0.8488030 \\ \mathrm{C} & 4.6511850 & -2.1221330 & -0.4202160 \\ \mathrm{H} & 2.5678380 & -1.9062940 & -0.8622830 \\ \mathrm{~N} & 5.8079140 & -1.6203850 & 0.0331860 \\ \mathrm{H} & 6.7008280 & 0.0569910 & 0.8380730 \\ \mathrm{H} & 4.6853830 & -3.1503450 & -0.7752080 \\ \mathrm{H} & -1.2452360 & -1.5732770 & 0.1407600 \\ & & & \\ & & & \end{array}$

10 (excited-state/ESIPT TS) G=-952.385511 au, $v(\mathrm{imag})=1137 i \mathrm{~cm}^{-1}$ 


$\begin{array}{lrrr}\text { C } & -3.1601250 & -0.8994610 & 0.0668900 \\ \text { C } & -3.7559840 & 0.3800530 & 0.0049820 \\ \text { C } & -5.1190360 & 0.5890320 & 0.0061010 \\ \text { C } & -5.9139230 & -0.5609580 & 0.0736940 \\ \text { C } & -5.3450550 & -1.8426290 & 0.1360550 \\ \text { C } & -3.9677060 & -2.0412720 & 0.1344200 \\ \text { C } & -1.5958980 & 0.6115090 & -0.0268010 \\ \text { H } & -3.5307570 & -3.0318620 & 0.1826400 \\ \text { N } & -1.8073770 & -0.7176070 & 0.0458170 \\ \text { H } & -5.5415880 & 1.5856390 & -0.0422180 \\ \text { O } & -2.7631720 & 1.3212710 & -0.0524750 \\ \text { H } & -6.0010970 & -2.7055220 & 0.1870850 \\ \text { C } & -0.3277060 & 1.2050480 & -0.0706180 \\ \text { C } & 0.8243340 & 0.2728690 & -0.0453120 \\ \text { C } & -0.0859560 & 2.5856700 & -0.1199250 \\ \text { C } & 2.1636570 & 0.7823470 & -0.0818240 \\ \text { C } & 1.2344590 & 3.0501850 & -0.1533760 \\ \text { H } & -0.9201450 & 3.2773130 & -0.1296090 \\ \text { C } & 2.3345580 & 2.1820280 & -0.1314580 \\ \text { O } & 0.5925420 & -0.9885500 & 0.0486380 \\ \text { H } & -6.9935020 & -0.4571380 & 0.0779710 \\ \text { H } & 3.3342420 & 2.5932080 & -0.2051680 \\ \text { H } & 1.4119420 & 4.1195970 & -0.2075510 \\ \mathrm{C} & 3.3167780 & -0.1165140 & -0.0241510 \\ \text { C } & 4.5225920 & 0.3208120 & 0.5570710 \\ \text { C } & 3.3247480 & -1.4183800 & -0.5601440 \\ \text { C } & 5.6215030 & -0.5258500 & 0.5819480 \\ \text { H } & 4.5930820 & 1.2995740 & 1.0190650 \\ \text { C } & 4.4846390 & -2.1771730 & -0.4861390 \\ \text { H } & 2.4492080 & -1.8265760 & -1.0467490 \\ \text { N } & 5.6288450 & -1.7628990 & 0.0722340 \\ \text { H } & 6.5487440 & -0.1910030 & 1.0420490 \\ \text { H } & 4.4950970 & -3.1800210 & -0.9077970 \\ \text { H } & -0.5657480 & -1.1653230 & 0.0661220 \\ & & & \end{array}$

10. $\mathrm{H}^{+}$(ground-state) $\mathrm{G}=-952.947570 \mathrm{au}$

$\begin{array}{lrrr}\text { C } & -3.2974130 & -0.9176790 & 0.0877530 \\ \text { C } & -3.8018020 & 0.3801070 & -0.0054170 \\ \text { C } & -5.1518870 & 0.6859110 & -0.0193170 \\ \text { C } & -6.0110020 & -0.4061950 & 0.0680860 \\ \text { C } & -5.5280530 & -1.7230000 & 0.1632990 \\ \text { C } & -4.1669870 & -2.0042660 & 0.1749780 \\ \text { C } & -1.6453640 & 0.4319760 & -0.0244950 \\ \text { H } & -3.7908580 & -3.0184310 & 0.2481950 \\ \text { N } & -1.9065240 & -0.8376890 & 0.0718710 \\ \text { H } & -5.5103230 & 1.7056840 & -0.0930770 \\ \text { O } & -2.7334760 & 1.2361760 & -0.0766460 \\ \text { H } & -6.2392730 & -2.5394040 & 0.2292940 \\ \text { C } & -0.3230010 & 1.0357020 & -0.0765210 \\ \text { C } & 0.8129370 & 0.1966260 & -0.0329240 \\ \text { C } & -0.1732150 & 2.4221530 & -0.1647680 \\ \text { C } & 2.0970130 & 0.7814060 & -0.0663530 \\ \text { C } & 1.0897220 & 2.9951210 & -0.2017680 \\ \text { H } & -1.0624520 & 3.0424200 & -0.2045490 \\ \text { C } & 2.2097890 & 2.1741330 & -0.1442670 \\ \text { O } & 0.7176480 & -1.1341480 & 0.0674850 \\ \text { H } & -0.2395850 & -1.3953580 & 0.1022550 \\ \text { H } & -7.0821110 & -0.2351920 & 0.0625050 \\ \text { H } & 3.1988350 & 2.6192270 & -0.1940070 \\ \text { H } & 1.2030360 & 4.0697300 & -0.2790770\end{array}$




$\begin{array}{lrrr}\text { C } & 3.3155590 & -0.0459670 & -0.0140010 \\ \text { C } & 4.4470690 & 0.4162390 & 0.6807400 \\ \text { C } & 3.4090800 & -1.2880410 & -0.6675440 \\ \text { C } & 5.6004680 & -0.3344650 & 0.7061000 \\ \text { H } & 4.4234710 & 1.3514180 & 1.2262860 \\ \text { C } & 4.5846020 & -2.0021430 & -0.6196830 \\ \text { H } & 2.5709120 & -1.6913980 & -1.2178940 \\ \text { N } & 5.6392760 & -1.5135480 & 0.0597790 \\ \text { H } & 6.4985680 & -0.0374150 & 1.2313690 \\ \text { H } & 4.7241950 & -2.9564730 & -1.1098880 \\ \text { H } & 6.4978650 & -2.0581030 & 0.0869400\end{array}$

10. $\mathrm{H}^{+}$(excited-state/enol) G= -952.824323 au

$\begin{array}{lrrr}\text { C } & -3.2527740 & -0.9138990 & 0.0802280 \\ \text { C } & -3.8069650 & 0.3719420 & -0.0107140 \\ \text { C } & -5.1670780 & 0.6259840 & -0.0255060 \\ \text { C } & -5.9809230 & -0.4995880 & 0.0579650 \\ \text { C } & -5.4491000 & -1.8026170 & 0.1502700 \\ \text { C } & -4.0833140 & -2.0367140 & 0.1635700 \\ \text { C } & -1.6581960 & 0.5105570 & -0.0284840 \\ \text { H } & -3.6687420 & -3.0354880 & 0.2339010 \\ \text { N } & -1.8801070 & -0.7793290 & 0.0649690 \\ \text { H } & -5.5644470 & 1.6310410 & -0.0965270 \\ \text { O } & -2.7757800 & 1.2677520 & -0.0783050 \\ \text { H } & -6.1325260 & -2.6423370 & 0.2123100 \\ \text { C } & -0.3661180 & 1.1287070 & -0.0844020 \\ \text { C } & 0.7863290 & 0.2623700 & -0.0316010 \\ \text { C } & -0.1933440 & 2.5135240 & -0.1451820 \\ \text { C } & 2.1402320 & 0.8023600 & -0.0674190 \\ \text { C } & 1.1069950 & 3.0277290 & -0.1466300 \\ \text { H } & -1.0566530 & 3.1666500 & -0.1735790 \\ \text { C } & 2.2405700 & 2.1953830 & -0.1182260 \\ \text { O } & 0.6542460 & -1.0201140 & 0.0954210 \\ \text { H } & -0.3400570 & -1.2754290 & 0.1129420 \\ \text { H } & -7.0577350 & -0.3707800 & 0.0519080 \\ \text { H } & 3.2204270 & 2.6542840 & -0.1754370 \\ \text { H } & 1.2500770 & 4.1021660 & -0.1819990 \\ \text { C } & 3.2919840 & -0.0624910 & -0.0118690 \\ \text { C } & 4.5159130 & 0.4253010 & 0.5539010 \\ \text { C } & 3.3367420 & -1.3848870 & -0.5607820 \\ \text { C } & 5.6361100 & -0.3485050 & 0.5798430 \\ \text { H } & 4.5596580 & 1.4029120 & 1.0182440 \\ \text { C } & 4.4865770 & -2.1159420 & -0.5220220 \\ \text { H } & 2.4751860 & -1.8182640 & -1.0479430 \\ \text { N } & 5.6205310 & -1.6052370 & 0.0452880 \\ \text { H } & 6.5707260 & -0.0255340 & 1.0187320 \\ \text { H } & 4.5666110 & -3.1112790 & -0.9379540 \\ \text { H } & 6.4649280 & -2.1623580 & 0.0590230\end{array}$

10. $\mathrm{H}^{+}$(excited-state/keto) G= $-952.839664 \mathrm{au}$

$\begin{array}{rrrr}\text { C } & -3.3579250 & -0.9380690 & 0.0714100 \\ \text { C } & -3.8157150 & 0.3782640 & -0.0057130 \\ \text { C } & -5.1543110 & 0.7175940 & -0.0262140 \\ \text { C } & -6.0444140 & -0.3538060 & 0.0362290 \\ \text { C } & -5.5981330 & -1.6827740 & 0.1137730 \\ \text { C } & -4.2452860 & -2.0080370 & 0.1332120 \\ \text { C } & -1.6245270 & 0.4541520 & -0.0086670 \\ \text { H } & -3.8990830 & -3.0327600 & 0.1920310\end{array}$




$\begin{array}{lrrr}\text { N } & -1.9772110 & -0.8390680 & 0.0669690 \\ \text { H } & -5.4809910 & 1.7484020 & -0.0864500 \\ \text { O } & -2.7295250 & 1.2172660 & -0.0536160 \\ \text { H } & -6.3301970 & -2.4814200 & 0.1598890 \\ \text { C } & -0.3390090 & 1.0437330 & -0.0554360 \\ \text { C } & 0.8333050 & 0.1507940 & -0.0285070 \\ \mathrm{C} & -0.1873690 & 2.4330190 & -0.1019070 \\ \mathrm{C} & 2.1732410 & 0.7686200 & -0.0731600 \\ \mathrm{C} & 1.1035740 & 2.9769120 & -0.1376250 \\ \mathrm{H} & -1.0585940 & 3.0775090 & -0.1046410 \\ \mathrm{C} & 2.2489100 & 2.1725670 & -0.1302960 \\ \mathrm{O} & 0.6744030 & -1.0750830 & 0.0664630 \\ \mathrm{H} & -7.1099050 & -0.1536360 & 0.0244820 \\ \mathrm{H} & 3.2158760 & 2.6550790 & -0.2108820 \\ \mathrm{H} & 1.2185670 & 4.0546100 & -0.1803970 \\ \mathrm{C} & 3.3542360 & -0.0548530 & -0.0196730 \\ \mathrm{C} & 4.5883740 & 0.5007570 & 0.4431060 \\ \mathrm{C} & 3.4048420 & -1.4166990 & -0.4467820 \\ \mathrm{C} & 5.7310170 & -0.2453100 & 0.4754940 \\ \mathrm{H} & 4.6344810 & 1.5136080 & 0.8222280 \\ \mathrm{C} & 4.5765240 & -2.1185330 & -0.4051730 \\ \mathrm{H} & 2.5241230 & -1.9079600 & -0.8312340 \\ \mathrm{~N} & 5.7197190 & -1.5358810 & 0.0506280 \\ \mathrm{H} & 6.6778460 & 0.1349730 & 0.8364230 \\ \mathrm{H} & 4.6609450 & -3.1463940 & -0.7326210 \\ \mathrm{H} & 6.5788140 & -2.0728510 & 0.0694440 \\ \mathrm{H} & -1.2724500 & -1.5719490 & 0.1113430\end{array}$

10. $\mathrm{H}^{+}$(excited-state/ESIPT TS) G= $-952.825785 \mathrm{au}, v(\mathrm{imag})=1166 i \mathrm{~cm}^{-1}$

$\begin{array}{lrrr}\text { C } & -3.1938790 & -0.9034890 & 0.0754940 \\ \text { C } & -3.7926670 & 0.3613940 & -0.0046720 \\ \text { C } & -5.1603830 & 0.5639000 & -0.0133260 \\ \text { C } & -5.9334790 & -0.5915810 & 0.0644520 \\ \text { C } & -5.3532290 & -1.8715790 & 0.1455110 \\ \text { C } & -3.9775100 & -2.0560900 & 0.1531170 \\ \text { C } & -1.6499630 & 0.6083090 & -0.0276110 \\ \text { H } & -3.5273520 & -3.0396530 & 0.2156260 \\ \text { N } & -1.8279300 & -0.6954100 & 0.0577580 \\ \text { H } & -5.5934750 & 1.5546540 & -0.0757910 \\ \text { O } & -2.7966330 & 1.3052350 & -0.0685430 \\ \text { H } & -6.0027990 & -2.7380200 & 0.2037540 \\ \text { C } & -0.3611030 & 1.2196750 & -0.0808350 \\ \text { C } & 0.7642090 & 0.3005820 & -0.0410090 \\ \text { C } & -0.1523720 & 2.5975730 & -0.1272080 \\ \text { C } & 2.1337150 & 0.8168430 & -0.0714430 \\ \text { C } & 1.1649260 & 3.0740290 & -0.1350460 \\ \text { H } & -0.9958720 & 3.2773670 & -0.1418190 \\ \text { C } & 2.2723620 & 2.2100560 & -0.1178970 \\ \text { O } & 0.5639990 & -0.9570920 & 0.0600080 \\ \text { H } & -0.5945670 & -1.1519400 & 0.0782580 \\ \text { H } & -7.0141740 & -0.5019970 & 0.0626650 \\ \text { H } & 3.2640690 & 2.6425430 & -0.1772350 \\ \text { H } & 1.3384220 & 4.1441930 & -0.1634380 \\ \text { C } & 3.2627160 & -0.0770280 & -0.0156210 \\ \text { C } & 4.5055890 & 0.3835690 & 0.5287930 \\ \text { C } & 3.2599380 & -1.4101370 & -0.5359430 \\ \text { C } & 5.6024520 & -0.4247400 & 0.5590950 \\ \text { H } & 4.5851340 & 1.3682190 & 0.9727450 \\ \text { C } & 4.3877920 & -2.1762990 & -0.4933680 \\ \text { H } & 2.3774970 & -1.8256320 & -1.0001240\end{array}$




$\begin{array}{llll}\mathrm{N} & 5.5416920 & -1.6886360 & 0.0504290 \\ \mathrm{H} & 6.5513520 & -0.1221120 & 0.9816430 \\ \mathrm{H} & 4.4329540 & -3.1825880 & -0.8877480 \\ \mathrm{H} & 6.3688500 & -2.2717890 & 0.0675420\end{array}$


11 (ground-state) G= $-1356.527677 \mathrm{au}$

\begin{tabular}{|c|c|c|c|}
\hline C & -2.9647630 & 0.0876080 & 0.0273720 \\
\hline C & -2.7671080 & -1.2928260 & 0.0205960 \\
\hline C & -3.8049390 & -2.2032620 & 0.0308150 \\
\hline C & -5.0885810 & -1.6578240 & 0.0483550 \\
\hline C & -5.3347580 & -0.2695830 & 0.0555240 \\
\hline C & -4.2511410 & 0.6150720 & 0.0449990 \\
\hline C & -0.8602660 & -0.2826280 & -0.0011210 \\
\hline $\mathrm{H}$ & -4.3932030 & 1.6910650 & 0.0500560 \\
\hline $\mathrm{N}$ & -1.7120750 & 0.6992540 & 0.0128840 \\
\hline $\mathrm{H}$ & -3.6355010 & -3.2735180 & 0.0255480 \\
\hline 0 & -1.4149280 & -1.5204370 & 0.0023740 \\
\hline C & 0.5893540 & -0.1658390 & -0.0212380 \\
\hline C & 1.1753980 & 1.1194470 & -0.0228310 \\
\hline C & 1.3911340 & -1.3102510 & -0.0260070 \\
\hline C & 2.5816990 & 1.2307560 & -0.0257940 \\
\hline C & 2.7778820 & -1.2116130 & -0.0249350 \\
\hline $\mathrm{H}$ & 0.9095450 & -2.2830010 & -0.0076670 \\
\hline C & 3.3475170 & 0.0690420 & -0.0211490 \\
\hline 0 & 0.4468310 & 2.2428390 & -0.0033460 \\
\hline $\mathrm{H}$ & -0.5150820 & 2.0105800 & 0.0105960 \\
\hline $\mathrm{H}$ & -5.9246500 & -2.3466600 & 0.0565980 \\
\hline $\mathrm{H}$ & 4.4291370 & 0.1678620 & -0.0599780 \\
\hline C & 3.6296980 & -2.4232430 & -0.0318120 \\
\hline C & 4.8520680 & -2.4603090 & 0.6460010 \\
\hline C & 3.2469630 & -3.5816960 & -0.7149610 \\
\hline C & 5.6141000 & -3.6228580 & 0.6083780 \\
\hline $\mathrm{H}$ & 5.2002990 & -1.6056620 & 1.2171220 \\
\hline C & 4.0812950 & -4.6937460 & -0.6857200 \\
\hline $\mathrm{H}$ & 2.3229670 & -3.6148250 & -1.2832440 \\
\hline $\mathrm{N}$ & 5.2511280 & -4.7331240 & -0.0407880 \\
\hline $\mathrm{H}$ & 6.5645870 & -3.6639740 & 1.1353490 \\
\hline $\mathrm{H}$ & 3.7951010 & -5.5988120 & -1.2165990 \\
\hline C & 3.2531430 & 2.5544440 & -0.0282080 \\
\hline C & 2.8293460 & 3.6102520 & -0.8410430 \\
\hline C & 4.3792900 & 2.7756950 & 0.7695220 \\
\hline C & 3.5411270 & 4.8043790 & -0.8195200 \\
\hline $\mathrm{H}$ & 1.9635100 & 3.5037200 & -1.4831570 \\
\hline C & 5.0178830 & 4.0103720 & 0.7170380 \\
\hline $\mathrm{H}$ & 4.7467710 & 2.0029040 & 1.4374640 \\
\hline $\mathrm{N}$ & 4.6196940 & 5.0211850 & -0.0597200 \\
\hline $\mathrm{H}$ & 3.2261390 & 5.6319430 & -1.4510510 \\
\hline $\mathrm{H}$ & 5.8925960 & 4.1949260 & 1.3365080 \\
\hline C & -6.7571670 & 0.3041200 & 0.0740210 \\
\hline C & -6.9400850 & 1.1680080 & 1.3334990 \\
\hline C & -6.9697340 & 1.1744830 & -1.1763390 \\
\hline C & -7.8277500 & -0.7919010 & 0.0838120 \\
\hline $\mathrm{H}$ & -6.7924440 & 0.5700480 & 2.2388180 \\
\hline $\mathrm{H}$ & -6.2325580 & 2.0025130 & 1.3577630 \\
\hline $\mathrm{H}$ & -7.9528020 & 1.5850590 & 1.3577470 \\
\hline $\mathrm{H}$ & -6.8435960 & 0.5811500 & -2.0879510 \\
\hline $\mathrm{H}$ & -7.9826600 & 1.5917400 & -1.1746050 \\
\hline $\mathrm{H}$ & -6.2627820 & 2.0090130 & -1.2130100 \\
\hline $\mathrm{H}$ & -8.8190600 & -0.3282290 & 0.0967630 \\
\hline $\mathrm{H}$ & -7.7667580 & -1.4250820 & -0.8075850 \\
\hline $\mathrm{H}$ & -7.7456330 & -1.4297830 & 0.9701350 \\
\hline
\end{tabular}

11 (excited-state/enol) G= $-1356.393027 \mathrm{au}$

$\begin{array}{llll}\text { C } & -2.9440270 & 0.0202960 & 0.0197850\end{array}$ 


\begin{tabular}{|c|c|c|c|}
\hline C & -2.7661920 & -1.3843860 & 0.0153390 \\
\hline C & -3.8161090 & -2.2740590 & 0.0292770 \\
\hline C & -5.0992870 & -1.7124880 & 0.0484090 \\
\hline C & -5.3276200 & -0.3184410 & 0.0534610 \\
\hline C & -4.2383520 & 0.5563940 & 0.0394170 \\
\hline C & -0.8297870 & -0.4061490 & -0.0126340 \\
\hline $\mathrm{H}$ & -4.3707740 & 1.6332550 & 0.0428590 \\
\hline $\mathrm{N}$ & -1.7162600 & 0.6062010 & 0.0019830 \\
\hline $\mathrm{H}$ & -3.6598810 & -3.3463410 & 0.0258140 \\
\hline 0 & -1.4281810 & -1.6448710 & -0.0044580 \\
\hline C & 0.5642750 & -0.2897360 & -0.0331530 \\
\hline C & 1.1386680 & 1.0621690 & -0.0417250 \\
\hline C & 1.4414220 & -1.3946000 & -0.0209910 \\
\hline C & 2.5462730 & 1.2561560 & -0.0522690 \\
\hline C & 2.8268210 & -1.1936460 & -0.0198320 \\
\hline $\mathrm{H}$ & 1.0242180 & -2.3924560 & 0.0391860 \\
\hline C & 3.3649820 & 0.1151120 & -0.0308920 \\
\hline 0 & 0.3464760 & 2.0979850 & -0.0001100 \\
\hline $\mathrm{H}$ & -0.6346180 & 1.7892030 & 0.0091920 \\
\hline $\mathrm{H}$ & -5.9433340 & -2.3912330 & 0.0595910 \\
\hline $\mathrm{H}$ & 4.4377040 & 0.2427580 & -0.1196540 \\
\hline C & 3.7399690 & -2.3504570 & -0.0241460 \\
\hline C & 5.0089580 & -2.2843840 & 0.5658460 \\
\hline C & 3.3749600 & -3.5675360 & -0.6141870 \\
\hline C & 5.8270830 & -3.4077850 & 0.5407510 \\
\hline $\mathrm{H}$ & 5.3470170 & -1.3828770 & 1.0652400 \\
\hline C & 4.2727500 & -4.6282940 & -0.5897950 \\
\hline $\mathrm{H}$ & 2.4184120 & -3.6814650 & -1.1124260 \\
\hline $\mathrm{N}$ & 5.4826610 & -4.5690030 & -0.0247630 \\
\hline $\mathrm{H}$ & 6.8102700 & -3.3718710 & 1.0035360 \\
\hline $\mathrm{H}$ & 4.0048470 & -5.5749110 & -1.0527670 \\
\hline C & 3.1361400 & 2.6015370 & -0.0419050 \\
\hline C & 2.5717500 & 3.7056800 & -0.7024160 \\
\hline C & 4.3568030 & 2.8252880 & 0.6170500 \\
\hline C & 3.2317060 & 4.9270940 & -0.6667270 \\
\hline $\mathrm{H}$ & 1.6446600 & 3.6143280 & -1.2532740 \\
\hline C & 4.9283700 & 4.0901950 & 0.5919260 \\
\hline $\mathrm{H}$ & 4.8411930 & 2.0281990 & 1.1713720 \\
\hline $\mathrm{N}$ & 4.3919400 & 5.1423240 & -0.0356010 \\
\hline $\mathrm{H}$ & 2.8025480 & 5.7829970 & -1.1830150 \\
\hline $\mathrm{H}$ & 5.8683870 & 4.2676940 & 1.1097690 \\
\hline C & -6.7445460 & 0.2670500 & 0.0733070 \\
\hline C & -6.9174230 & 1.1349360 & 1.3318220 \\
\hline C & -6.9508910 & 1.1380270 & -1.1780510 \\
\hline C & -7.8265870 & -0.8177950 & 0.0862640 \\
\hline $\mathrm{H}$ & -6.7738610 & 0.5368350 & 2.2376980 \\
\hline $\mathrm{H}$ & -6.2019030 & 1.9624480 & 1.3533530 \\
\hline $\mathrm{H}$ & -7.9264740 & 1.5608270 & 1.3567610 \\
\hline $\mathrm{H}$ & -6.8310990 & 0.5421620 & -2.0888660 \\
\hline $\mathrm{H}$ & -7.9603560 & 1.5636730 & -1.1753860 \\
\hline $\mathrm{H}$ & -6.2363670 & 1.9657780 & -1.2164290 \\
\hline $\mathrm{H}$ & -8.8129590 & -0.3437020 & 0.1002090 \\
\hline $\mathrm{H}$ & -7.7734970 & -1.4526610 & -0.8044020 \\
\hline $\mathrm{H}$ & -7.7495470 & -1.4552860 & 0.9732680 \\
\hline
\end{tabular}

11 (excited-state/keto) G= -1356.408204 au

$\begin{array}{rrrr}\text { C } & -3.0179190 & 0.1343800 & 0.0378620 \\ \text { C } & -2.7962850 & -1.2481190 & 0.0099310 \\ \text { C } & -3.8270900 & -2.1596210 & -0.0039590 \\ \text { C } & -5.1225650 & -1.6273240 & 0.0115020\end{array}$




\begin{tabular}{|c|c|c|c|}
\hline C & -5.3797060 & -0.2462000 & 0.0392500 \\
\hline C & -4.3040500 & 0.6561030 & 0.0532040 \\
\hline C & -0.8219740 & -0.2764310 & 0.0188650 \\
\hline $\mathrm{H}$ & -4.4599910 & 1.7291040 & 0.0747110 \\
\hline$N$ & -1.7632270 & 0.7015680 & 0.0441970 \\
\hline $\mathrm{H}$ & -3.6442250 & -3.2272380 & -0.0256270 \\
\hline $\mathrm{O}$ & -1.4456900 & -1.4841240 & -0.0002440 \\
\hline C & 0.5851890 & -0.1861700 & 0.0039910 \\
\hline C & 1.2014880 & 1.1658590 & -0.0005700 \\
\hline C & 1.3830170 & -1.3247250 & 0.0256390 \\
\hline C & 2.6513750 & 1.2428570 & -0.0161700 \\
\hline C & 2.8006050 & -1.2121410 & 0.0113740 \\
\hline $\mathrm{H}$ & 0.9175590 & -2.3016550 & 0.0942750 \\
\hline C & 3.4016340 & 0.0508090 & -0.0016220 \\
\hline 0 & 0.4581280 & 2.1661220 & 0.0442210 \\
\hline $\mathrm{H}$ & -5.9505500 & -2.3252310 & 0.0012190 \\
\hline $\mathrm{H}$ & 4.4796200 & 0.1122310 & -0.1005070 \\
\hline C & 3.6297650 & -2.4274020 & -0.0129080 \\
\hline C & 4.9215270 & -2.4479000 & 0.5325860 \\
\hline C & 3.1656920 & -3.6224350 & -0.5801300 \\
\hline C & 5.6625420 & -3.6214000 & 0.4823860 \\
\hline $\mathrm{H}$ & 5.3361840 & -1.5709130 & 1.0176900 \\
\hline C & 3.9919510 & -4.7405410 & -0.5815180 \\
\hline $\mathrm{H}$ & 2.1867470 & -3.6788680 & -1.0435800 \\
\hline N & 5.2230190 & -4.7613320 & -0.0625230 \\
\hline $\mathrm{H}$ & 6.6622170 & -3.6481120 & 0.9094790 \\
\hline $\mathrm{H}$ & 3.6434300 & -5.6689950 & -1.0277760 \\
\hline C & 3.3261000 & 2.5380490 & -0.0316660 \\
\hline C & 2.7670280 & 3.7040140 & -0.5920350 \\
\hline C & 4.6232610 & 2.6766880 & 0.5026520 \\
\hline C & 3.5001310 & 4.8822210 & -0.5895540 \\
\hline $\mathrm{H}$ & 1.7817290 & 3.6847210 & -1.0365900 \\
\hline C & 5.2629250 & 3.9066010 & 0.4561650 \\
\hline $\mathrm{H}$ & 5.1185350 & 1.8409250 & 0.9852870 \\
\hline N & 4.7317250 & 5.0118880 & -0.0795370 \\
\hline $\mathrm{H}$ & 3.0706810 & 5.7793840 & -1.0309950 \\
\hline $\mathrm{H}$ & 6.2601740 & 4.0103360 & 0.8789930 \\
\hline C & -6.8054010 & 0.3161720 & 0.0537830 \\
\hline C & -7.0054020 & 1.1599380 & 1.3245930 \\
\hline C & -7.0129820 & 1.2031540 & -1.1860620 \\
\hline C & -7.8666770 & -0.7886320 & 0.0379450 \\
\hline $\mathrm{H}$ & -6.8598020 & 0.5499240 & 2.2220640 \\
\hline $\mathrm{H}$ & -6.3067230 & 2.0010800 & 1.3672500 \\
\hline $\mathrm{H}$ & -8.0222250 & 1.5666580 & 1.3459020 \\
\hline $\mathrm{H}$ & -6.8733430 & 0.6241790 & -2.1048280 \\
\hline $\mathrm{H}$ & -8.0296530 & 1.6108360 & -1.1871330 \\
\hline $\mathrm{H}$ & -6.3139140 & 2.0448770 & -1.2041970 \\
\hline $\mathrm{H}$ & -8.8616040 & -0.3330100 & 0.0498240 \\
\hline $\mathrm{H}$ & -7.7929570 & -1.4071050 & -0.8626450 \\
\hline $\mathrm{H}$ & -7.7861780 & -1.4390950 & 0.9151150 \\
\hline $\mathrm{H}$ & -1.4809140 & 1.6782850 & 0.0620990 \\
\hline
\end{tabular}

11 (excited-state/ESIPT TS) G= $-1356.394407 \mathrm{au}, v(\mathrm{imag})=1101 i \mathrm{~cm}^{-1}$

$\begin{array}{lrrr}\text { C } & -2.9289120 & -0.0701800 & 0.0187250 \\ \text { C } & -2.7629930 & -1.4711660 & 0.0140400 \\ \text { C } & -3.8228220 & -2.3484480 & 0.0281500 \\ \text { C } & -5.1004870 & -1.7742850 & 0.0476520 \\ \text { C } & -5.3118560 & -0.3817220 & 0.0528800 \\ \text { C } & -4.2098790 & 0.4825950 & 0.0385510 \\ \text { C } & -0.8038470 & -0.5374200 & -0.0136110\end{array}$




\begin{tabular}{|c|c|c|c|}
\hline $\mathrm{H}$ & -4.3312000 & 1.5606480 & 0.0421380 \\
\hline N & -1.6786850 & 0.4847520 & 0.0019790 \\
\hline H & -3.6768580 & -3.4222030 & 0.0245430 \\
\hline & -1.4225770 & -1.7541610 & -0.0056170 \\
\hline C & 0.5886980 & -0.3800980 & -0.0357720 \\
\hline C & 1.0799510 & 1.0141580 & -0.0467430 \\
\hline$c$ & 1.5118440 & -1.4329310 & -0.0179200 \\
\hline C & 2.4884040 & 1.2741070 & -0.0501700 \\
\hline 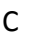 & 2.8940820 & -1.1581960 & -0.0155480 \\
\hline H & 1.1501400 & -2.4524940 & 0.0467290 \\
\hline C & 3.3626860 & 0.1722590 & -0.0255670 \\
\hline O & 0.2238770 & 1.9720290 & -0.0150120 \\
\hline H & -5.9510610 & -2.4446480 & 0.0590320 \\
\hline H & 4.4276670 & 0.3505680 & -0.1178540 \\
\hline C & 3.8603330 & -2.2692180 & -0.0178210 \\
\hline C & 5.1291560 & -2.1387490 & 0.5627910 \\
\hline C & 3.5503760 & -3.5068040 & -0.5974890 \\
\hline C & 6.0003120 & -3.2211340 & 0.5377360 \\
\hline $\mathrm{H}$ & 5.4262690 & -1.2194930 & 1.0556270 \\
\hline C & 4.4990200 & -4.5224800 & -0.5742320 \\
\hline $\mathrm{H}$ & 2.5970550 & -3.6712920 & -1.0876170 \\
\hline $\mathrm{N}$ & 5.7084870 & -4.4012280 & -0.0187030 \\
\hline $\mathrm{H}$ & 6.9836960 & -3.1350720 & 0.9933690 \\
\hline $\mathrm{H}$ & 4.2737810 & -5.4838270 & -1.0294760 \\
\hline C & 3.0084070 & 2.6428530 & -0.0430420 \\
\hline C & 2.3573070 & 3.7314800 & -0.6523620 \\
\hline C & 4.2476570 & 2.9219100 & 0.5631760 \\
\hline C & 2.9550700 & 4.9841010 & -0.6221610 \\
\hline H & 1.4110990 & 3.6026820 & -1.1605120 \\
\hline C & 4.7501400 & 4.2150570 & 0.5383240 \\
\hline $\mathrm{H}$ & 4.7994920 & 2.1448930 & 1.0813860 \\
\hline N & 4.1318390 & 5.2497950 & -0.0418610 \\
\hline $\mathrm{H}$ & 2.4578930 & 5.8253880 & -1.1002370 \\
\hline H & 5.7029900 & 4.4319300 & 1.0163910 \\
\hline C & -6.7203590 & 0.2236090 & 0.0732090 \\
\hline C & -6.8810680 & 1.0941360 & 1.3314180 \\
\hline C & -6.9158010 & 1.0962760 & -1.1786280 \\
\hline C & -7.8164260 & -0.8469430 & 0.0874180 \\
\hline H & -6.7451210 & 0.4944620 & 2.2374300 \\
\hline $\mathrm{H}$ & -6.1548650 & 1.9123290 & 1.3527720 \\
\hline H & -7.8843060 & 1.5334880 & 1.3566460 \\
\hline H & -6.8046340 & 0.4981110 & -2.0890280 \\
\hline $\mathrm{H}$ & -7.9194220 & 1.5354810 & -1.1756770 \\
\hline H & -6.1905150 & 1.9145930 & -1.2185210 \\
\hline H & -8.7965660 & -0.3601060 & 0.1017250 \\
\hline H & -7.7720240 & -1.4829640 & -0.8028860 \\
\hline $\mathrm{H}$ & -7.7469440 & -1.4848930 & 0.9747110 \\
\hline & -0.8524160 & 1.5196290 & -0.0035930 \\
\hline
\end{tabular}

11.2 $\mathrm{H}^{+}$(ground-state) $\mathrm{G}=-1357.367921 \mathrm{au}$

$\begin{array}{rrrr}\text { C } & -2.9982990 & 0.0562240 & 0.0242900 \\ \text { C } & -2.8024240 & -1.3243860 & 0.0249520 \\ \text { C } & -3.8415650 & -2.2335440 & 0.0414800 \\ \text { C } & -5.1225860 & -1.6839710 & 0.0574150 \\ \text { C } & -5.3671400 & -0.2943870 & 0.0572430 \\ \text { C } & -4.2830240 & 0.5882000 & 0.0404510 \\ \text { C } & -0.8971240 & -0.3208840 & -0.0034600 \\ \text { H } & -4.4227510 & 1.6643220 & 0.0398410 \\ \text { N } & -1.7430430 & 0.6639020 & 0.0055300 \\ \text { H } & -3.6748590 & -3.3041060 & 0.0421350\end{array}$




\begin{tabular}{|c|c|c|c|}
\hline 0 & -1.4484120 & -1.5549390 & 0.0070770 \\
\hline C & 0.5529940 & -0.1933940 & -0.0233460 \\
\hline C & 1.1210880 & 1.1027180 & -0.0292130 \\
\hline C & 1.3695570 & -1.3189270 & -0.0298250 \\
\hline C & 2.5282040 & 1.2317240 & -0.0227730 \\
\hline C & 2.7603710 & -1.1978640 & -0.0195820 \\
\hline $\mathrm{H}$ & 0.8984550 & -2.2967510 & -0.0106610 \\
\hline C & 3.3182860 & 0.0859590 & -0.0031010 \\
\hline 0 & 0.3822630 & 2.2067530 & -0.0158000 \\
\hline $\mathrm{H}$ & -0.5845060 & 1.9626980 & -0.0035660 \\
\hline $\mathrm{H}$ & -5.9604350 & -2.3704510 & 0.0704110 \\
\hline $\mathrm{H}$ & 4.3978250 & 0.2020230 & -0.0387250 \\
\hline C & 3.6195840 & -2.3940550 & -0.0292760 \\
\hline C & 4.8709650 & -2.3929540 & 0.6094530 \\
\hline C & 3.2125790 & -3.5730390 & -0.6764770 \\
\hline C & 5.6583570 & -3.5217630 & 0.5871420 \\
\hline $\mathrm{H}$ & 5.2259570 & -1.5254480 & 1.1515030 \\
\hline C & 4.0308170 & -4.6801840 & -0.6705720 \\
\hline $\mathrm{H}$ & 2.2711210 & -3.6274580 & -1.2092110 \\
\hline$N$ & 5.2214190 & -4.6256960 & -0.0459980 \\
\hline $\mathrm{H}$ & 6.6262670 & -3.5860400 & 1.0659480 \\
\hline $\mathrm{H}$ & 3.7785640 & -5.6135230 & -1.1561110 \\
\hline C & 3.1680130 & 2.5634940 & -0.0306920 \\
\hline C & 2.6755040 & 3.6207300 & -0.8132990 \\
\hline C & 4.3195340 & 2.7885970 & 0.7390080 \\
\hline C & 3.3279970 & 4.8338450 & -0.8108420 \\
\hline $\mathrm{H}$ & 1.7930160 & 3.4993930 & -1.4261070 \\
\hline C & 4.9371430 & 4.0198200 & 0.7135580 \\
\hline $\mathrm{H}$ & 4.7235840 & 2.0144560 & 1.3799490 \\
\hline $\mathrm{N}$ & 4.4282520 & 4.9993460 & -0.0536940 \\
\hline $\mathrm{H}$ & 3.0091570 & 5.6850300 & -1.3973750 \\
\hline $\mathrm{H}$ & 5.8198460 & 4.2614500 & 1.2907440 \\
\hline C & -6.7887630 & 0.2809390 & 0.0748490 \\
\hline C & -6.9676570 & 1.1509240 & 1.3307810 \\
\hline C & -7.0009660 & 1.1455060 & -1.1796430 \\
\hline C & -7.8602610 & -0.8140550 & 0.0913090 \\
\hline $\mathrm{H}$ & -6.8206760 & 0.5567960 & 2.2386660 \\
\hline $\mathrm{H}$ & -6.2586370 & 1.9843690 & 1.3503050 \\
\hline $\mathrm{H}$ & -7.9793850 & 1.5699010 & 1.3538750 \\
\hline $\mathrm{H}$ & -6.8778310 & 0.5474830 & -2.0885270 \\
\hline $\mathrm{H}$ & -8.0130680 & 1.5642490 & -1.1779200 \\
\hline $\mathrm{H}$ & -6.2929120 & 1.9789320 & -1.2214320 \\
\hline $\mathrm{H}$ & -8.8507120 & -0.3489540 & 0.1035380 \\
\hline $\mathrm{H}$ & -7.8016940 & -1.4512310 & -0.7973700 \\
\hline $\mathrm{H}$ & -7.7780500 & -1.4476280 & 0.9806570 \\
\hline $\mathrm{H}$ & 5.8137660 & -5.4531100 & -0.0512970 \\
\hline $\mathrm{H}$ & 4.8936550 & 5.9045800 & -0.0593420 \\
\hline
\end{tabular}

11.2 $\mathrm{H}^{+}$(excited-state/enol) $\mathrm{G}=-1357.242232 \mathrm{au}$

$\begin{array}{lrrr}\text { C } & -2.9878670 & 0.0078690 & 0.0201210 \\ \text { C } & -2.8058750 & -1.3898800 & -0.0101490 \\ \text { C } & -3.8559280 & -2.2842820 & -0.0092920 \\ \text { C } & -5.1266900 & -1.7142030 & 0.0239690 \\ \text { C } & -5.3598440 & -0.3128690 & 0.0549720 \\ \text { C } & -4.2738120 & 0.5567200 & 0.0536030 \\ \text { C } & -0.8873960 & -0.4118340 & -0.0272730 \\ \text { H } & -4.3983120 & 1.6339140 & 0.0767810 \\ \text { N } & -1.7475610 & 0.5894410 & 0.0074850\end{array}$




\begin{tabular}{|c|c|c|c|}
\hline $\mathrm{H}$ & -3.7049240 & -3.3564120 & -0.0328040 \\
\hline 0 & -1.4650580 & -1.6379840 & -0.0387030 \\
\hline C & 0.5274720 & -0.2858380 & -0.0579200 \\
\hline C & 1.0787420 & 1.0487850 & -0.0439710 \\
\hline C & 1.3819430 & -1.4026070 & -0.0626360 \\
\hline C & 2.5156750 & 1.2635650 & -0.0486360 \\
\hline C & 2.7632540 & -1.1955850 & -0.0330700 \\
\hline $\mathrm{H}$ & 0.9531170 & -2.3963230 & -0.0234260 \\
\hline C & 3.3091850 & 0.1105350 & -0.0290620 \\
\hline 0 & 0.3093950 & 2.0870360 & 0.0197220 \\
\hline $\mathrm{H}$ & -0.6768730 & 1.8041390 & 0.0266870 \\
\hline $\mathrm{H}$ & -5.9747100 & -2.3878960 & 0.0259600 \\
\hline $\mathrm{H}$ & 4.3842910 & 0.2191940 & -0.1076010 \\
\hline C & 3.6743030 & -2.3541190 & -0.0187190 \\
\hline C & 4.9132760 & -2.2874020 & 0.6386110 \\
\hline C & 3.3234390 & -3.5530180 & -0.6604100 \\
\hline C & 5.7479110 & -3.3840320 & 0.6408190 \\
\hline $\mathrm{H}$ & 5.2211180 & -1.3977110 & 1.1739320 \\
\hline C & 4.1902810 & -4.6237090 & -0.6336310 \\
\hline $\mathrm{H}$ & 2.3921250 & -3.6488540 & -1.2045780 \\
\hline $\mathrm{N}$ & 5.3658290 & -4.5087200 & 0.0102420 \\
\hline $\mathrm{H}$ & 6.7112280 & -3.4044810 & 1.1331370 \\
\hline $\mathrm{H}$ & 3.9882390 & -5.5727410 & -1.1124900 \\
\hline C & 3.0839140 & 2.5864530 & -0.0372940 \\
\hline C & 2.4519080 & 3.7442980 & -0.5875370 \\
\hline C & 4.3966490 & 2.7843460 & 0.4966860 \\
\hline C & 3.0883880 & 4.9520270 & -0.5851960 \\
\hline $\mathrm{H}$ & 1.4773650 & 3.6876400 & -1.0485440 \\
\hline C & 4.9844070 & 4.0148020 & 0.4865220 \\
\hline $\mathrm{H}$ & 4.9324350 & 1.9703910 & 0.9683000 \\
\hline N & 4.3351370 & 5.0816970 & -0.0526840 \\
\hline $\mathrm{H}$ & 2.6526820 & 5.8478930 & -1.0067770 \\
\hline $\mathrm{H}$ & 5.9643390 & 4.2069160 & 0.9025220 \\
\hline C & -6.7779170 & 0.2673200 & 0.0882510 \\
\hline C & -6.9451350 & 1.1143710 & 1.3618730 \\
\hline C & -6.9857950 & 1.1570250 & -1.1498850 \\
\hline C & -7.8557010 & -0.8218100 & 0.0869470 \\
\hline $\mathrm{H}$ & -6.7973510 & 0.5029840 & 2.2578560 \\
\hline $\mathrm{H}$ & -6.2330760 & 1.9446710 & 1.3929540 \\
\hline $\mathrm{H}$ & -7.9550270 & 1.5361630 & 1.3957670 \\
\hline $\mathrm{H}$ & -6.8688460 & 0.5760470 & -2.0703930 \\
\hline $\mathrm{H}$ & -7.9954590 & 1.5805930 & -1.1362640 \\
\hline $\mathrm{H}$ & -6.2733620 & 1.9871100 & -1.1765660 \\
\hline $\mathrm{H}$ & -8.8420280 & -0.3493170 & 0.1137390 \\
\hline $\mathrm{H}$ & -7.8074810 & -1.4398580 & -0.8157310 \\
\hline $\mathrm{H}$ & -7.7751850 & -1.4739160 & 0.9628980 \\
\hline $\mathrm{H}$ & 5.9957320 & -5.3085310 & 0.0196140 \\
\hline $\mathrm{H}$ & 4.7887010 & 5.9874710 & -0.0645760 \\
\hline
\end{tabular}

\section{2 $\mathrm{H}^{+}$(excited-state/keto) $\mathrm{G}=-1357.260776 \mathrm{au}$}

$\begin{array}{rrrr}\text { C } & -3.0576920 & 0.1344380 & 0.0434790 \\ \text { C } & -2.8347810 & -1.2434650 & 0.0047760 \\ \text { C } & -3.8630890 & -2.1606440 & -0.0157530 \\ \text { C } & -5.1512410 & -1.6250520 & 0.0042950 \\ \text { C } & -5.4121500 & -0.2390460 & 0.0428230 \\ \text { C } & -4.3409530 & 0.6621310 & 0.0639950 \\ \text { C } & -0.8710080 & -0.2729000 & 0.0170260 \\ \text { H } & -4.4952580 & 1.7349810 & 0.0939960 \\ \text { N } & -1.7950460 & 0.7006730 & 0.0508940 \\ \text { H } & -3.6805410 & -3.2278140 & -0.0458160\end{array}$




\begin{tabular}{|c|c|c|c|}
\hline 0 & -1.4800950 & -1.4699230 & -0.0093200 \\
\hline C & 0.5405200 & -0.1839270 & -0.0103090 \\
\hline C & 1.1539340 & 1.1583450 & -0.0055790 \\
\hline C & 1.3280710 & -1.3363530 & -0.0129300 \\
\hline C & 2.6270380 & 1.2436570 & -0.0303350 \\
\hline C & 2.7330650 & -1.2171280 & -0.0157810 \\
\hline $\mathrm{H}$ & 0.8536390 & -2.3091960 & 0.0441040 \\
\hline C & 3.3574940 & 0.0445580 & -0.0248240 \\
\hline 0 & 0.4354660 & 2.1620570 & 0.0557080 \\
\hline $\mathrm{H}$ & -5.9811280 & -2.3203860 & -0.0111810 \\
\hline $\mathrm{H}$ & 4.4352660 & 0.0843970 & -0.1298930 \\
\hline C & 3.5602630 & -2.4339170 & -0.0193900 \\
\hline C & 4.8237590 & -2.4494410 & 0.5961860 \\
\hline C & 3.1097150 & -3.6137770 & -0.6361710 \\
\hline C & 5.5810040 & -3.5997660 & 0.5832030 \\
\hline $\mathrm{H}$ & 5.2082510 & -1.5793240 & 1.1134960 \\
\hline C & 3.9027850 & -4.7396740 & -0.6270350 \\
\hline $\mathrm{H}$ & 2.1575260 & -3.6535900 & -1.1502940 \\
\hline$N$ & 5.1039830 & -4.7023090 & -0.0224930 \\
\hline $\mathrm{H}$ & 6.5556890 & -3.6808640 & 1.0454950 \\
\hline $\mathrm{H}$ & 3.6204650 & -5.6754590 & -1.0908120 \\
\hline C & 3.2820480 & 2.5263120 & -0.0345880 \\
\hline C & 2.6792320 & 3.7251450 & -0.5182980 \\
\hline C & 4.6296020 & 2.6432630 & 0.4243050 \\
\hline C & 3.3776400 & 4.9006680 & -0.5301900 \\
\hline $\mathrm{H}$ & 1.6719860 & 3.7224780 & -0.9054190 \\
\hline C & 5.2809310 & 3.8444450 & 0.3996590 \\
\hline $\mathrm{H}$ & 5.1525470 & 1.7950600 & 0.8476490 \\
\hline$N$ & 4.6574320 & 4.9505400 & -0.0764440 \\
\hline $\mathrm{H}$ & 2.9636460 & 5.8287120 & -0.9016660 \\
\hline $\mathrm{H}$ & 6.2945620 & 3.9775280 & 0.7543480 \\
\hline C & -6.8397230 & 0.3176430 & 0.0600220 \\
\hline C & -7.0408130 & 1.1525950 & 1.3366270 \\
\hline C & -7.0465220 & 1.2112360 & -1.1754450 \\
\hline C & -7.8962250 & -0.7916360 & 0.0365410 \\
\hline $\mathrm{H}$ & -6.8930700 & 0.5379550 & 2.2303740 \\
\hline $\mathrm{H}$ & -6.3468880 & 1.9974930 & 1.3837940 \\
\hline $\mathrm{H}$ & -8.0593070 & 1.5539380 & 1.3594880 \\
\hline $\mathrm{H}$ & -6.9054670 & 0.6383270 & -2.0976380 \\
\hline $\mathrm{H}$ & -8.0641100 & 1.6155880 & -1.1738060 \\
\hline $\mathrm{H}$ & -6.3504890 & 2.0556460 & -1.1875310 \\
\hline $\mathrm{H}$ & -8.8919630 & -0.3387380 & 0.0516490 \\
\hline $\mathrm{H}$ & -7.8219670 & -1.4029960 & -0.8687830 \\
\hline $\mathrm{H}$ & -7.8147630 & -1.4474220 & 0.9095830 \\
\hline $\mathrm{H}$ & 5.6738810 & -5.5455780 & -0.0208440 \\
\hline $\mathrm{H}$ & 5.1582010 & 5.8323080 & -0.0987700 \\
\hline $\mathrm{H}$ & -1.5256850 & 1.6813190 & 0.0758080 \\
\hline
\end{tabular}

11.2H $\mathrm{H}^{+}$(excited-state/ESIPT TS) G=--1357.244199 au, $v(\mathrm{imag})=1060 i \mathrm{~cm}^{-1}$

$\begin{array}{lrrr}\text { C } & -2.9683300 & -0.0792220 & 0.0156840 \\ \text { C } & -2.7979710 & -1.4730230 & -0.0132910 \\ \text { C } & -3.8561240 & -2.3574240 & -0.0071710 \\ \text { C } & -5.1230530 & -1.7779900 & 0.0294460 \\ \text { C } & -5.3401210 & -0.3793260 & 0.0587240 \\ \text { C } & -4.2429010 & 0.4828440 & 0.0522330 \\ \text { C } & -0.8569230 & -0.5376820 & -0.0373310 \\ \text { H } & -4.3594490 & 1.5609490 & 0.0740200 \\ \text { N } & -1.7056060 & 0.4738120 & -0.0007660 \\ \text { H } & -3.7124760 & -3.4307110 & -0.0293940 \\ \text { O } & -1.4532960 & -1.7428490 & -0.0458970\end{array}$




\begin{tabular}{|c|c|c|c|}
\hline C & 0.5550610 & -0.3724680 & -0.0719430 \\
\hline C & 1.0274310 & 1.0038380 & -0.0585760 \\
\hline C & 1.4587650 & -1.4362110 & -0.0747180 \\
\hline C & 2.4617010 & 1.2839400 & -0.0582560 \\
\hline C & 2.8353970 & -1.1582580 & -0.0484930 \\
\hline H & 1.0855090 & -2.4526270 & -0.0318730 \\
\hline C & 3.3150910 & 0.1709840 & -0.0480630 \\
\hline $\mathrm{O}$ & 0.1911470 & 1.9650050 & -0.0043220 \\
\hline $\mathrm{H}$ & -0.8804280 & 1.5299500 & 0.0041900 \\
\hline $\mathrm{H}$ & -5.9761410 & -2.4450510 & 0.0355180 \\
\hline $\mathrm{H}$ & 4.3826950 & 0.3349910 & -0.1311680 \\
\hline C & 3.7928090 & -2.2770260 & -0.0209010 \\
\hline C & 5.0265050 & -2.1546690 & 0.6390500 \\
\hline C & 3.4931910 & -3.4947230 & -0.6534850 \\
\hline C & 5.9027450 & -3.2181910 & 0.6559570 \\
\hline $\mathrm{H}$ & 5.2970120 & -1.2483640 & 1.1664090 \\
\hline C & 4.4007900 & -4.5302280 & -0.6122280 \\
\hline $\mathrm{H}$ & 2.5699390 & -3.6327560 & -1.2023290 \\
\hline $\mathrm{N}$ & 5.5682810 & -4.3634340 & 0.0354300 \\
\hline $\mathrm{H}$ & 6.8635910 & -3.1956630 & 1.1530710 \\
\hline $\mathrm{H}$ & 4.2371720 & -5.4907310 & -1.0824170 \\
\hline C & 2.9597910 & 2.6329660 & -0.0378430 \\
\hline C & 2.2550750 & 3.7596490 & -0.5644250 \\
\hline C & 4.2681730 & 2.8959340 & 0.4801020 \\
\hline C & 2.8254450 & 5.0002370 & -0.5607360 \\
\hline $\mathrm{H}$ & 1.2758430 & 3.6514260 & -1.0058200 \\
\hline C & 4.7875960 & 4.1573590 & 0.4716140 \\
\hline $\mathrm{H}$ & 4.8545300 & 2.1085350 & 0.9370570 \\
\hline$N$ & 4.0730080 & 5.1905560 & -0.0488370 \\
\hline $\mathrm{H}$ & 2.3346540 & 5.8756360 & -0.9642330 \\
\hline $\mathrm{H}$ & 5.7625940 & 4.3998380 & 0.8732730 \\
\hline C & -6.7508770 & 0.2187330 & 0.0951240 \\
\hline C & -6.9040120 & 1.0730610 & 1.3654750 \\
\hline C & -6.9536240 & 1.1047820 & -1.1463220 \\
\hline C & -7.8412160 & -0.8577380 & 0.1030330 \\
\hline $\mathrm{H}$ & -6.7602320 & 0.4636320 & 2.2634820 \\
\hline $\mathrm{H}$ & -6.1824150 & 1.8952700 & 1.3911850 \\
\hline $\mathrm{H}$ & -7.9087640 & 1.5068970 & 1.4012940 \\
\hline $\mathrm{H}$ & -6.8468650 & 0.5178260 & -2.0643000 \\
\hline $\mathrm{H}$ & -7.9582810 & 1.5401630 & -1.1312460 \\
\hline $\mathrm{H}$ & -6.2317840 & 1.9264750 & -1.1802850 \\
\hline $\mathrm{H}$ & -8.8220860 & -0.3740380 & 0.1323440 \\
\hline $\mathrm{H}$ & -7.8038550 & -1.4800580 & -0.7972090 \\
\hline $\mathrm{H}$ & -7.7636680 & -1.5072520 & 0.9811690 \\
\hline $\mathrm{H}$ & 6.2294390 & -5.1379050 & 0.0549220 \\
\hline $\mathrm{H}$ & 4.4783820 & 6.1188950 & -0.0626560 \\
\hline
\end{tabular}


12 (ground-state) G= $-992.214363 \mathrm{au}$

$\begin{array}{lrrr}\text { C } & -4.0514850 & -0.0628120 & 0.0234940 \\ \text { C } & -3.3445260 & -1.2653230 & 0.0648620 \\ \text { C } & -3.9429540 & -2.5124500 & 0.1199840 \\ \text { C } & -5.3353010 & -2.5054390 & 0.1323960 \\ \text { C } & -6.0704590 & -1.3079650 & 0.0911490 \\ \text { C } & -5.4458460 & -0.0675310 & 0.0361170 \\ \text { C } & -1.9666010 & 0.3927260 & -0.0124710 \\ \text { H } & -6.0091200 & 0.8582590 & 0.0041880 \\ \text { N } & -3.1262160 & 0.9777480 & -0.0257320 \\ \text { H } & -3.3642890 & -3.4278950 & 0.1515630 \\ \text { O } & -2.0077470 & -0.9609580 & 0.0408510 \\ \text { H } & -7.1540690 & -1.3564140 & 0.1028900 \\ \text { C } & -0.6733500 & 1.0562270 & -0.0500550 \\ \text { C } & -0.6240270 & 2.4694940 & -0.0926140 \\ \text { C } & 0.5110110 & 0.3197910 & -0.0371860 \\ \text { C } & 0.6266740 & 3.1039870 & -0.1158500 \\ \text { C } & 1.7528610 & 0.9528850 & -0.0520760 \\ \text { H } & 0.4413400 & -0.7621200 & 0.0183580 \\ \text { C } & 1.7882740 & 2.3608100 & -0.0890740 \\ \text { O } & -1.7126620 & 3.2386360 & -0.1126930 \\ \text { H } & -2.5219830 & 2.6704710 & -0.0915840 \\ \text { H } & -5.8660880 & -3.4504170 & 0.1753210 \\ \text { H } & 2.7411460 & 2.8791080 & -0.1315280 \\ \text { H } & 0.6509240 & 4.1868240 & -0.1607870 \\ \text { C } & 2.9945370 & 0.1685100 & -0.0258650 \\ \text { C } & 4.1707500 & 0.6769580 & 0.5500510 \\ \text { C } & 3.0619830 & -1.1223570 & -0.5759950 \\ \text { C } & 5.3234320 & -0.0739940 & 0.5564490 \\ \text { H } & 4.1920990 & 1.6524350 & 1.0199130 \\ \text { C } & 4.2393040 & -1.8332960 & -0.5404680 \\ \text { H } & 2.2062400 & -1.5717390 & -1.0645310 \\ \text { N } & 5.3510330 & -1.3092170 & 0.0151900 \\ \text { H } & 6.2477220 & 0.2785630 & 0.9975640 \\ \text { H } & 4.3342390 & -2.8270300 & -0.9602450 \\ \text { C } & 6.5907400 & -2.1071180 & 0.0729850 \\ \text { H } & 7.4440820 & -1.4341100 & 0.0165200 \\ \text { H } & 6.6100200 & -2.6652830 & 1.0095630 \\ \text { H } & 6.6071700 & -2.7904120 & -0.7736610\end{array}$

12 (excited-state/enol) G= $-992.085897 \mathrm{au}$

$\begin{array}{lrrr}\text { C } & -4.0650080 & -0.0323550 & 0.0034490 \\ \text { C } & -3.3898700 & -1.2598120 & 0.0487210 \\ \text { C } & -4.0248540 & -2.4894030 & 0.0831810 \\ \text { C } & -5.4148660 & -2.4377750 & 0.0699490 \\ \text { C } & -6.1185000 & -1.2161300 & 0.0243950 \\ \text { C } & -5.4633300 & 0.0043870 & -0.0094270 \\ \text { C } & -1.9653120 & 0.3526910 & 0.0067170 \\ \text { H } & -5.9978620 & 0.9464760 & -0.0447080 \\ \text { N } & -3.1158060 & 0.9717700 & -0.0222110 \\ \text { H } & -3.4747340 & -3.4217460 & 0.1179050 \\ \text { O } & -2.0481180 & -0.9957740 & 0.0500500 \\ \text { H } & -7.2027080 & -1.2378790 & 0.0157720 \\ \text { C } & -0.6728360 & 0.9902940 & -0.0055940 \\ \text { C } & -0.6358560 & 2.4287800 & -0.0513930 \\ \text { C } & 0.5075830 & 0.2644340 & 0.0328920 \\ \text { C } & 0.6284400 & 3.0875640 & -0.0634540 \\ \text { C } & 1.7752030 & 0.9116500 & -0.0010070\end{array}$




$\begin{array}{llll}\text { H } & 0.4417840 & -0.8140420 & 0.1052000 \\ \mathrm{C} & 1.7765430 & 2.3605450 & -0.0405900 \\ \mathrm{O} & -1.7090080 & 3.1713730 & -0.0854910 \\ \mathrm{H} & -2.5311080 & 2.5930240 & -0.0717230 \\ \mathrm{H} & -5.9752630 & -3.3659550 & 0.0954430 \\ \mathrm{H} & 2.7223020 & 2.8874810 & -0.0793320 \\ \mathrm{H} & 0.6337780 & 4.1705230 & -0.1029190 \\ \mathrm{C} & 3.0065790 & 0.1745300 & -0.0120470 \\ \mathrm{C} & 4.2773460 & 0.7804540 & 0.2636360 \\ \mathrm{C} & 3.0419570 & -1.2357500 & -0.2877500 \\ \mathrm{C} & 5.4236090 & 0.0442420 & 0.2611020 \\ \mathrm{H} & 4.3631330 & 1.8304880 & 0.5130670 \\ \mathrm{C} & 4.2186340 & -1.9214990 & -0.2919840 \\ \mathrm{H} & 2.1426850 & -1.7830090 & -0.5408180 \\ \mathrm{~N} & 5.4125530 & -1.3004480 & -0.0247240 \\ \mathrm{H} & 6.3938930 & 0.4731650 & 0.4776750 \\ \mathrm{H} & 4.2761320 & -2.9807430 & -0.5099410 \\ \mathrm{C} & 6.6465990 & -2.0734700 & 0.0460520 \\ \mathrm{H} & 7.4814820 & -1.4470850 & -0.2703870 \\ \mathrm{H} & 6.8230320 & -2.4236280 & 1.0682410 \\ \mathrm{H} & 6.5708530 & -2.9313810 & -0.6224930\end{array}$

12 (excited-state/keto) G= - $-992.095273 \mathrm{au}$

$\begin{array}{lccc}\mathrm{C} & -4.1145390 & -0.0273970 & 0.0059630 \\ \mathrm{C} & -3.3944190 & -1.2269360 & 0.0517190 \\ \mathrm{C} & -3.9945380 & -2.4698180 & 0.0913280 \\ \mathrm{C} & -5.3901720 & -2.4654830 & 0.0837910 \\ \mathrm{C} & -6.1248470 & -1.2701490 & 0.0382250 \\ \mathrm{C} & -5.5071250 & -0.0234810 & -0.0014600 \\ \mathrm{C} & -1.9314750 & 0.4071750 & -0.0019200 \\ \mathrm{H} & -6.0728240 & 0.8995450 & -0.0371600 \\ \mathrm{~N} & -3.1597230 & 0.9664290 & -0.0253290 \\ \mathrm{H} & -3.4121560 & -3.3823500 & 0.1256550 \\ \mathrm{O} & -2.0542340 & -0.9364940 & 0.0471800 \\ \mathrm{H} & -7.2082570 & -1.3186180 & 0.0335910 \\ \mathrm{C} & -0.6642550 & 1.0282830 & -0.0073120 \\ \mathrm{C} & -0.6232280 & 2.5019110 & -0.0810300 \\ \mathrm{C} & 0.5184560 & 0.2889830 & 0.0693440 \\ \mathrm{C} & 0.6988630 & 3.1150660 & -0.0948590 \\ \mathrm{C} & 1.7879250 & 0.9307650 & 0.0273620 \\ \mathrm{H} & 0.4540100 & -0.7867270 & 0.1802040 \\ \mathrm{C} & 1.8286900 & 2.3631150 & -0.0406110 \\ \mathrm{O} & -1.6549280 & 3.1817750 & -0.1327060 \\ \mathrm{H} & -5.9194120 & -3.4112860 & 0.1137420 \\ \mathrm{H} & 2.7896570 & 2.8625260 & -0.0944430 \\ \mathrm{H} & 0.7304660 & 4.1962520 & -0.1685980 \\ \mathrm{C} & 3.0181910 & 0.1770220 & 0.0079740 \\ \mathrm{C} & 4.2775550 & 0.7412310 & 0.3541620 \\ \mathrm{C} & 3.0334590 & -1.2072180 & -0.3381330 \\ \mathrm{C} & 5.4153510 & -0.0141780 & 0.3382090 \\ \mathrm{H} & 4.3673100 & 1.7725980 & 0.6717250 \\ \mathrm{C} & 4.2039270 & -1.9149510 & -0.3485850 \\ \mathrm{H} & 2.1300660 & -1.7214970 & -0.6404850 \\ \mathrm{~N} & 5.3867040 & -1.3363900 & -0.0128140 \\ \mathrm{H} & 6.3870220 & 0.3831500 & 0.6050290 \\ \mathrm{H} & 4.2463400 & -2.9615870 & -0.6241390 \\ \mathrm{C} & 6.6347550 & -2.1015220 & -0.0078640 \\ \mathrm{H} & 7.3482630 & -1.6396730 & -0.6933530 \\ \mathrm{H} & 7.0520910 & -2.1170910 & 1.0012180 \\ \mathrm{H} & 6.4277900 & -3.1197140 & -0.3319360 \\ & & & \end{array}$


12 (excited-state/ESIPT TS) G= $-992.083908 \mathrm{au}, v(\mathrm{imag})=1343 i \mathrm{~cm}^{-1}$

$\begin{array}{lrrr}\text { C } & -4.0832860 & 0.0157510 & 0.0037550 \\ \text { C } & -3.4872250 & -1.2518330 & 0.0620640 \\ \text { C } & -4.2025510 & -2.4340420 & 0.1100490 \\ \text { C } & -5.5876720 & -2.2921530 & 0.0973350 \\ \text { C } & -6.2063820 & -1.0289890 & 0.0394980 \\ \text { C } & -5.4727010 & 0.1483580 & -0.0083770 \\ \text { C } & -1.9271650 & 0.2377300 & 0.0034050 \\ \text { H } & -5.9453480 & 1.1222020 & -0.0530460 \\ \text { N } & -3.0494740 & 0.9314690 & -0.0324050 \\ \text { H } & -3.7132430 & -3.3993010 & 0.1543390 \\ \text { O } & -2.1226900 & -1.0877850 & 0.0606760 \\ \text { H } & -7.2895850 & -0.9770010 & 0.0321320 \\ \text { C } & -0.6410680 & 0.8581500 & -0.0106190 \\ \text { C } & -0.6702800 & 2.3118450 & -0.0755820 \\ \text { C } & 0.5623840 & 0.1685940 & 0.0504130 \\ \text { C } & 0.5920430 & 3.0079140 & -0.0925160 \\ \text { C } & 1.8009980 & 0.8692780 & 0.0014040 \\ \text { H } & 0.5432930 & -0.9104680 & 0.1491170 \\ \text { C } & 1.7564510 & 2.3123210 & -0.0557090 \\ \text { O } & -1.7668690 & 2.9596160 & -0.1193080 \\ \text { H } & -2.6728390 & 2.1411010 & -0.0872090 \\ \text { H } & -6.2074780 & -3.1812180 & 0.1330270 \\ \text { H } & 2.6902830 & 2.8608190 & -0.1061890 \\ \text { H } & 0.5662880 & 4.0896910 & -0.1505160 \\ \text { C } & 3.0649070 & 0.1801940 & -0.0223910 \\ \text { C } & 4.2951700 & 0.8003980 & 0.3600940 \\ \text { C } & 3.1549060 & -1.2007640 & -0.3996170 \\ \text { C } & 5.4642060 & 0.0992050 & 0.3628600 \\ \text { H } & 4.3294430 & 1.8303160 & 0.6942130 \\ \text { C } & 4.3535590 & -1.8507380 & -0.3978870 \\ \text { H } & 2.2837800 & -1.7467760 & -0.7408050 \\ \text { N } & 5.5084860 & -1.2212920 & -0.0191600 \\ \text { H } & 6.4089370 & 0.5356380 & 0.6619050 \\ \text { H } & 4.4549920 & -2.8867090 & -0.6969160 \\ \text { C } & 6.7673790 & -1.9533160 & 0.0685960 \\ \text { H } & 7.5765310 & -1.3309490 & -0.3179130 \\ \text { H } & 6.9806750 & -2.2206250 & 1.1081640 \\ \text { H } & 6.6956180 & -2.8600310 & -0.5309600\end{array}$


15 (ground-state) G= $-1223.099622 \mathrm{au}$

\begin{tabular}{|c|c|c|c|}
\hline C & 5.7606940 & -0.6896410 & -0.0060260 \\
\hline C & 4.8036550 & -1.7052930 & -0.0123750 \\
\hline C & 5.1090080 & -3.0554800 & -0.0231730 \\
\hline C & 6.4678200 & -3.3593060 & -0.0270930 \\
\hline C & 7.4513520 & -2.3549350 & -0.0204120 \\
\hline C & 7.1189640 & -1.0054290 & -0.0098770 \\
\hline C & 3.8295410 & 0.2192590 & 0.0030870 \\
\hline $\mathrm{H}$ & 7.8743680 & -0.2278010 & -0.0048270 \\
\hline $\mathrm{N}$ & 5.0902120 & 0.5318730 & 0.0037720 \\
\hline $\mathrm{H}$ & 4.3409130 & -3.8196180 & -0.0283560 \\
\hline 0 & 3.5682110 & -1.1106050 & -0.0061040 \\
\hline $\mathrm{H}$ & 8.4968820 & -2.6438650 & -0.0237460 \\
\hline C & 2.7160110 & 1.1543010 & 0.0133140 \\
\hline C & 2.9813290 & 2.5441550 & 0.0101900 \\
\hline C & 1.3982180 & 0.6986460 & 0.0204050 \\
\hline C & 1.9024290 & 3.4407190 & 0.0067950 \\
\hline C & 0.3271720 & 1.5915490 & 0.0119500 \\
\hline $\mathrm{H}$ & 1.2273280 & -0.3731250 & -0.0018050 \\
\hline C & 0.6051110 & 2.9730380 & 0.0005610 \\
\hline 0 & 4.2133390 & 3.0523360 & 0.0102500 \\
\hline $\mathrm{H}$ & 4.8763420 & 2.3182850 & 0.0118270 \\
\hline $\mathrm{H}$ & 6.7746530 & -4.3996420 & -0.0355280 \\
\hline $\mathrm{H}$ & -0.2086790 & 3.6911210 & 0.0212450 \\
\hline $\mathrm{H}$ & 2.1189630 & 4.5028750 & 0.0154780 \\
\hline C & -1.0566280 & 1.1026420 & 0.0122390 \\
\hline C & -2.0968490 & 1.8448200 & -0.5733300 \\
\hline C & -1.4033590 & -0.1273550 & 0.5979690 \\
\hline C & -3.3849560 & 1.3650420 & -0.5684250 \\
\hline $\mathrm{H}$ & -1.9035850 & 2.7844000 & -1.0757190 \\
\hline C & -2.7072120 & -0.5619890 & 0.5880620 \\
\hline $\mathrm{H}$ & -0.6656560 & -0.7369020 & 1.1050120 \\
\hline$N$ & -3.6815950 & 0.1772330 & 0.0084240 \\
\hline $\mathrm{H}$ & -4.2094990 & 1.8837660 & -1.0408420 \\
\hline $\mathrm{H}$ & -3.0254400 & -1.4827470 & 1.0605570 \\
\hline C & -5.0459530 & -0.3036000 & 0.0057750 \\
\hline C & -6.0620480 & 0.5353450 & 0.4476030 \\
\hline C & -5.3085700 & -1.5959840 & -0.4377530 \\
\hline C & -7.3702230 & 0.0633190 & 0.4366650 \\
\hline $\mathrm{H}$ & -5.8341830 & 1.5297540 & 0.8190060 \\
\hline C & -6.6216340 & -2.0486290 & -0.4342400 \\
\hline $\mathrm{H}$ & -4.5040060 & -2.2289780 & -0.7992930 \\
\hline C & -7.6706960 & -1.2286340 & -0.0034320 \\
\hline $\mathrm{H}$ & -8.1685690 & 0.7094720 & 0.7885010 \\
\hline $\mathrm{H}$ & -6.8360880 & -3.0544750 & -0.7829040 \\
\hline C & -9.0913530 & -1.7265730 & -0.0363550 \\
\hline $\mathrm{H}$ & -9.4860940 & -1.6881790 & -1.0571440 \\
\hline $\mathrm{H}$ & -9.1518380 & -2.7647860 & 0.3002530 \\
\hline $\mathrm{H}$ & -9.7394750 & -1.1170730 & 0.5970060 \\
\hline
\end{tabular}

15 (excited-state/enol) G= -1222.977836 au

$\begin{array}{llll}\text { C } & -5.7932670 & -0.6523620 & -0.0058350 \\ \text { C } & -4.8605610 & -1.6955150 & -0.0147320 \\ \text { C } & -5.2016120 & -3.0371080 & -0.0260890 \\ \text { C } & -6.5676290 & -3.3010110 & -0.0282200 \\ \text { C } & -7.5271220 & -2.2692760 & -0.0193480 \\ \text { C } & -7.1625560 & -0.9311420 & -0.0081010 \\ \text { C } & -3.8354180 & 0.1972070 & 0.0008910 \\ \text { H } & -7.8961840 & -0.1333550 & -0.0012950\end{array}$




\begin{tabular}{lrrr} 
N & -5.0921080 & 0.5435160 & 0.0039320 \\
H & -4.4548780 & -3.8218040 & -0.0327950 \\
O & -3.6112560 & -1.1355360 & -0.0104660 \\
H & -8.5790310 & -2.5332080 & -0.0214990 \\
C & -2.7130660 & 1.1092450 & 0.0082650 \\
C & -2.9875820 & 2.5182030 & 0.0233430 \\
$\mathrm{C}$ & -1.4056120 & 0.6573880 & 0.0102060 \\
$\mathrm{C}$ & -1.8981330 & 3.4299160 & 0.0361420 \\
$\mathrm{C}$ & -0.3059330 & 1.5592570 & 0.0173250 \\
$\mathrm{H}$ & -1.2400940 & -0.4128290 & 0.0284310 \\
$\mathrm{C}$ & -0.6156330 & 2.9689790 & 0.0331700 \\
$\mathrm{O}$ & -4.2008410 & 3.0116290 & 0.0238620 \\
$\mathrm{H}$ & -4.8743820 & 2.2696010 & 0.0160010 \\
$\mathrm{H}$ & -6.9038450 & -4.3321240 & -0.0369570 \\
$\mathrm{H}$ & 0.1902810 & 3.6927400 & 0.0183090 \\
$\mathrm{H}$ & -2.1251390 & 4.4897800 & 0.0393010 \\
$\mathrm{C}$ & 1.0509080 & 1.0975850 & 0.0032210 \\
$\mathrm{C}$ & 2.1680790 & 1.9580730 & 0.2612650 \\
$\mathrm{C}$ & 1.3914090 & -0.2708380 & -0.2649440 \\
$\mathrm{C}$ & 3.4446580 & 1.4950190 & 0.2580280 \\
$\mathrm{H}$ & 2.0299620 & 2.9997830 & 0.5214010 \\
$\mathrm{C}$ & 2.6790410 & -0.6996720 & -0.2727930 \\
$\mathrm{H}$ & 0.6329690 & -0.9977330 & -0.5266470 \\
$\mathrm{~N}$ & 3.7358040 & 0.1619250 & -0.0076290 \\
$\mathrm{H}$ & 4.2855980 & 2.1195070 & 0.5224650 \\
$\mathrm{H}$ & 2.9487540 & -1.7097770 & -0.5455060 \\
$\mathrm{C}$ & 5.0609010 & -0.3000210 & -0.0086590 \\
$\mathrm{C}$ & 6.1091320 & 0.5505930 & -0.4028160 \\
$\mathrm{C}$ & 5.3527130 & -1.6199370 & 0.3835890 \\
$\mathrm{C}$ & 7.4126030 & 0.0829770 & -0.3988480 \\
$\mathrm{H}$ & 5.8980900 & 1.5524500 & -0.7601970 \\
$\mathrm{C}$ & 6.6625720 & -2.0643610 & 0.3785730 \\
$\mathrm{H}$ & 4.5630530 & -2.2749940 & 0.7346900 \\
$\mathrm{H}$ & 7.7199360 & -1.2269480 & -0.0076980 \\
$\mathrm{H}$ & 8.2109900 & 0.7437250 & -0.7236350 \\
$\mathrm{H}$ & 6.8765420 & -3.0805170 & 0.6973730 \\
& 9.1391610 & -1.7186120 & 0.0222360 \\
& 9.5294410 & -1.7015210 & 1.0466290 \\
\hline & -7894720 & -1.0921350 & -0.5923570
\end{tabular}

15 (excited-state/keto) G= -1222.983942 au

$\begin{array}{llll}\text { C } & -5.8322800 & -0.6669020 & -0.0197620 \\ \text { C } & -4.8646720 & -1.6749830 & 0.0105670 \\ \text { C } & -5.1714280 & -3.0214600 & 0.0270460 \\ \text { C } & -6.5321180 & -3.3265460 & 0.0111510 \\ \text { C } & -7.5150660 & -2.3239150 & -0.0201380 \\ \text { C } & -7.1905460 & -0.9711420 & -0.0360090 \\ \text { C } & -3.8040520 & 0.2418220 & -0.0036080 \\ \text { H } & -7.9465890 & -0.1958360 & -0.0597540 \\ \text { N } & -5.1189930 & 0.5166280 & -0.0274420 \\ \text { H } & -4.4007040 & -3.7820050 & 0.0509130 \\ \text { O } & -3.6222490 & -1.0903000 & 0.0195880 \\ \text { H } & -8.5602300 & -2.6125910 & -0.0318840 \\ \text { C } & -2.7097370 & 1.1369590 & 0.0087530 \\ \text { C } & -3.0072570 & 2.5796560 & -0.0239310 \\ \text { C } & -1.3934180 & 0.6778100 & 0.0678100 \\ \text { C } & -1.8529550 & 3.4744740 & -0.0070370 \\ \text { C } & -0.3000180 & 1.5834610 & 0.0565090 \\ \text { H } & -1.2168830 & -0.3889940 & 0.1413240\end{array}$




$\begin{array}{lrrr}\text { C } & -0.5864700 & 2.9944740 & 0.0338080 \\ \text { O } & -4.1644510 & 3.0131300 & -0.0660750 \\ \text { H } & -6.8380030 & -4.3666050 & 0.0230110 \\ \text { H } & 0.2386130 & 3.6979510 & 0.0074110 \\ \text { H } & -2.0640120 & 4.5372840 & -0.0452760 \\ \text { C } & 1.0657150 & 1.1253060 & 0.0302480 \\ \text { C } & 2.1685070 & 1.9412610 & 0.4152260 \\ \text { C } & 1.3969170 & -0.2076680 & -0.3643130 \\ \text { C } & 3.4447850 & 1.4629720 & 0.4035480 \\ \text { H } & 2.0226150 & 2.9493570 & 0.7834510 \\ \text { C } & 2.6885490 & -0.6405910 & -0.3835040 \\ \text { H } & 0.6353940 & -0.8911630 & -0.7192770 \\ \text { N } & 3.7260250 & 0.1727130 & 0.0048770 \\ \text { H } & 4.2881840 & 2.0452620 & 0.7485000 \\ \text { H } & 2.9668560 & -1.6230530 & -0.7410320 \\ \text { C } & 5.0649590 & -0.3065960 & -0.0040300 \\ \text { C } & 6.1054140 & 0.5376890 & -0.4003980 \\ \text { C } & 5.3381210 & -1.6236540 & 0.3790700 \\ \text { C } & 7.4092440 & 0.0592480 & -0.4068330 \\ \text { H } & 5.8942940 & 1.5468360 & -0.7388580 \\ \text { C } & 6.6469280 & -2.0835350 & 0.3576160 \\ \text { H } & 4.5386940 & -2.2719720 & 0.7230910 \\ \text { C } & 7.7057770 & -1.2539750 & -0.0295980 \\ \text { H } & 8.2111550 & 0.7174670 & -0.7284670 \\ \text { H } & 6.8526310 & -3.1056210 & 0.6629780 \\ \text { C } & 9.1236680 & -1.7593480 & -0.0158630 \\ \text { H } & 9.5299000 & -1.7413850 & 1.0014970 \\ \text { H } & 9.1782590 & -2.7916700 & -0.3717880 \\ \text { H } & 9.7699740 & -1.1413490 & -0.6434380 \\ \text { H } & -5.4471670 & 1.4811570 & -0.0505180\end{array}$

15 (excited-state/ESIPT TS) G= $-1222.975058 \mathrm{au}, v(\mathrm{imag})=1300 i \mathrm{~cm}^{-1}$

$\begin{array}{lrrr}\text { C } & -5.8634910 & -0.5324030 & -0.0005990 \\ \text { C } & -5.0280780 & -1.6553340 & 0.0089680 \\ \text { C } & -5.4936380 & -2.9573660 & 0.0173630 \\ \text { C } & -6.8791300 & -3.0927070 & 0.0158140 \\ \text { C } & -7.7363260 & -1.9766770 & 0.0062940 \\ \text { C } & -7.2505570 & -0.6759890 & -0.0021000 \\ \text { C } & -3.7932540 & 0.1139510 & -0.0011450 \\ \text { H } & -7.9070170 & 0.1859000 & -0.0093500 \\ \text { N } & -5.0273230 & 0.5718480 & -0.0072790 \\ \text { H } & -4.8223470 & -3.8073270 & 0.0247470 \\ \text { O } & -3.7217730 & -1.2219410 & 0.0082480 \\ \text { H } & -8.8083970 & -2.1396410 & 0.0055380 \\ \text { C } & -2.6559420 & 0.9844980 & -0.0020960 \\ \text { C } & -2.9768610 & 2.4034670 & -0.0090870 \\ \text { C } & -1.3440170 & 0.5436660 & 0.0197660 \\ \text { C } & -1.8748980 & 3.3292300 & -0.0055800 \\ \text { C } & -0.2652440 & 1.4703120 & 0.0115980 \\ \text { H } & -1.1536440 & -0.5225520 & 0.0679210 \\ \text { C } & -0.5941980 & 2.8763450 & 0.0035060 \\ \text { O } & -4.1821640 & 2.8193220 & -0.0200790 \\ \text { H } & -7.3096190 & -4.0879230 & 0.0221890 \\ \text { H } & 0.2109820 & 3.6017920 & -0.0284540 \\ \text { H } & -2.1103300 & 4.3870780 & -0.0234220 \\ \text { C } & 1.1056940 & 1.0375490 & -0.0052370 \\ \text { C } & 2.1932330 & 1.8876370 & 0.3689020 \\ \text { C } & 1.4733100 & -0.2951720 & -0.3820090 \\ \text { C } & 3.4782650 & 1.4427660 & 0.3694130 \\ \text { H } & 2.0222880 & 2.8964750 & 0.7245560\end{array}$




$\begin{array}{lrrr}\mathrm{C} & 2.7707130 & -0.7001060 & -0.3905340 \\ \mathrm{H} & 0.7315320 & -1.0013760 & -0.7349860 \\ \mathrm{~N} & 3.7979310 & 0.1468910 & -0.0100030 \\ \mathrm{H} & 4.3015730 & 2.0499390 & 0.7177260 \\ \mathrm{H} & 3.0699230 & -1.6769290 & -0.7438930 \\ \mathrm{C} & 5.1365360 & -0.2971080 & -0.0058640 \\ \mathrm{C} & 6.1757250 & 0.5904470 & -0.3248850 \\ \mathrm{C} & 5.4379080 & -1.6295840 & 0.3127730 \\ \mathrm{C} & 7.4872340 & 0.1427600 & -0.3193150 \\ \mathrm{H} & 5.9544360 & 1.6097940 & -0.6227510 \\ \mathrm{C} & 6.7570990 & -2.0559980 & 0.3079340 \\ \mathrm{H} & 4.6499820 & -2.3164200 & 0.6028600 \\ \mathrm{C} & 7.8070340 & -1.1835360 & -0.0024500 \\ \mathrm{H} & 8.2817860 & 0.8344250 & -0.5846880 \\ \mathrm{H} & 6.9784160 & -3.0877130 & 0.5660640 \\ \mathrm{C} & 9.2375020 & -1.6469330 & 0.0279490 \\ \mathrm{H} & 9.6844560 & -1.4534750 & 1.0099070 \\ \mathrm{H} & 9.3109270 & -2.7203590 & -0.1625830 \\ \mathrm{H} & 9.8393640 & -1.1184510 & -0.7159140 \\ \mathrm{H} & -4.9147890 & 1.8142600 & -0.0161230\end{array}$


16 (ground-state) G= $-1396.206243 a u$

\begin{tabular}{|c|c|c|c|}
\hline$C$ & 7.1099310 & 0.9664660 & -0.0060990 \\
\hline - & 6.0755020 & 1.9002900 & -0.0809600 \\
\hline C & 6.2725160 & 3.2682400 & -0.1598440 \\
\hline C & 7.6026910 & 3.6796850 & -0.1605300 \\
\hline C & 8.6626300 & 2.7593610 & -0.0856540 \\
\hline C & 8.4385740 & 1.3898500 & -0.0072640 \\
\hline C & 5.2572980 & -0.0931320 & 0.0214960 \\
\hline $\mathrm{H}$ & 9.2533470 & 0.6769560 & 0.0502300 \\
\hline $\mathrm{N}$ & 6.5386540 & -0.3028700 & 0.0582390 \\
\hline $\mathrm{H}$ & 5.4461460 & 3.9666500 & -0.2172660 \\
\hline 0 & 4.8915240 & 1.2093650 & -0.0621450 \\
\hline $\mathrm{H}$ & 9.6816770 & 3.1311520 & -0.0896050 \\
\hline C & 4.2211490 & -1.1125560 & 0.0573130 \\
\hline C & 4.5943020 & -2.4740660 & 0.1465440 \\
\hline C & 2.8716500 & -0.7639460 & 0.0082320 \\
\hline C & 3.5886750 & -3.4510340 & 0.1875650 \\
\hline C & 1.8732150 & -1.7356800 & 0.0548840 \\
\hline $\mathrm{H}$ & 2.6167680 & 0.2901120 & -0.0355500 \\
\hline C & 2.2585840 & -3.0877990 & 0.1480550 \\
\hline 0 & 5.8625960 & -2.8826350 & 0.1930550 \\
\hline $\mathrm{H}$ & 6.4658880 & -2.0998180 & 0.1575310 \\
\hline $\mathrm{H}$ & 7.8260030 & 4.7394660 & -0.2205550 \\
\hline $\mathrm{H}$ & 1.5043080 & -3.8683820 & 0.1567430 \\
\hline $\mathrm{H}$ & 3.8874460 & -4.4915560 & 0.2437820 \\
\hline C & 0.4552290 & -1.3560820 & 0.0091670 \\
\hline C & -0.5379880 & -2.1503040 & 0.6066850 \\
\hline C & 0.0267690 & -0.1823410 & -0.6339200 \\
\hline C & -1.8586170 & -1.7702640 & 0.5608150 \\
\hline $\mathrm{H}$ & -0.2852380 & -3.0502660 & 1.1535380 \\
\hline C & -1.3073090 & 0.1476320 & -0.6699560 \\
\hline $\mathrm{H}$ & 0.7256640 & 0.4620260 & -1.1528180 \\
\hline $\mathrm{N}$ & -2.2367790 & -0.6361870 & -0.0743840 \\
\hline $\mathrm{H}$ & -2.6486990 & -2.3282410 & 1.0468630 \\
\hline $\mathrm{H}$ & -1.6849050 & 1.0162140 & -1.1941890 \\
\hline C & -3.6285240 & -0.2625630 & -0.1195070 \\
\hline C & -4.5883380 & -1.2008400 & -0.4827980 \\
\hline C & -4.0105190 & 1.0352060 & 0.2109640 \\
\hline C & -5.9253760 & -0.8439610 & -0.5260360 \\
\hline $\mathrm{H}$ & -4.2946760 & -2.2102420 & -0.7550670 \\
\hline C & -5.3445120 & 1.3962010 & 0.1658980 \\
\hline $\mathrm{H}$ & -3.2702460 & 1.7601180 & 0.5368120 \\
\hline C & -6.3495130 & 0.4744900 & -0.2245340 \\
\hline $\mathrm{H}$ & -6.6393950 & -1.6040070 & -0.8103860 \\
\hline $\mathrm{H}$ & -5.6010790 & 2.4060410 & 0.4559840 \\
\hline$N$ & -7.6706940 & 0.8391560 & -0.3204790 \\
\hline C & -8.6598940 & -0.2251830 & -0.5265420 \\
\hline C & -10.0934830 & 0.2594540 & -0.6865420 \\
\hline $\mathrm{H}$ & -8.6079200 & -0.9505500 & 0.2996660 \\
\hline $\mathrm{H}$ & -8.3862260 & -0.7632430 & -1.4399140 \\
\hline $\mathrm{H}$ & -10.7213860 & -0.6064850 & -0.9101560 \\
\hline $\mathrm{H}$ & -10.4832400 & 0.7257960 & 0.2219480 \\
\hline $\mathrm{H}$ & -10.1872700 & 0.9665630 & -1.5152640 \\
\hline C & -8.1077110 & 2.1074410 & 0.2572860 \\
\hline C & -8.2049310 & 2.0718420 & 1.7814350 \\
\hline $\mathrm{H}$ & -9.0751070 & 2.3597770 & -0.1759120 \\
\hline $\mathrm{H}$ & -7.4288510 & 2.8959380 & -0.0755670 \\
\hline $\mathrm{H}$ & -8.5273070 & 3.0423370 & 2.1687320 \\
\hline $\mathrm{H}$ & -8.9301510 & 1.3165970 & 2.1010460 \\
\hline $\mathrm{H}$ & -7.2387830 & 1.8240670 & 2.2315370 \\
\hline
\end{tabular}


16 (excited-state/enol) G $=-1396.096717$ au

\begin{tabular}{|c|c|c|c|}
\hline$C$ & 7.1172020 & 0.9790120 & -0.0053630 \\
\hline & 6.0786490 & 1.9091330 & -0.0763370 \\
\hline$C$ & 6.2701880 & 3.2783360 & -0.1453260 \\
\hline C & 7.5985870 & 3.6959500 & -0.1400800 \\
\hline C & 8.6623670 & 2.7797150 & -0.0693120 \\
\hline C & 8.4440580 & 1.4087280 & -0.0009180 \\
\hline C & 5.2678730 & -0.0889700 & 0.0138160 \\
\hline $\mathrm{H}$ & 9.2619280 & 0.6991150 & 0.0537270 \\
\hline $\mathrm{N}$ & 6.5516890 & -0.2921130 & 0.0507690 \\
\hline $\mathrm{H}$ & 5.4407740 & 3.9734410 & -0.1993050 \\
\hline 0 & 4.8982350 & 1.2136130 & -0.0636000 \\
\hline $\mathrm{H}$ & 9.6798420 & 3.1559200 & -0.0681940 \\
\hline C & 4.2364170 & -1.1120370 & 0.0487930 \\
\hline C & 4.6228420 & -2.4716420 & 0.1429510 \\
\hline C & 2.8873920 & -0.7692500 & 0.0049660 \\
\hline C & 3.6175430 & -3.4519120 & 0.1907000 \\
\hline C & 1.8713660 & -1.7420280 & 0.0514150 \\
\hline $\mathrm{H}$ & 2.6375780 & 0.2850640 & -0.0366500 \\
\hline C & 2.2886190 & -3.0981040 & 0.1475760 \\
\hline 0 & 5.8943190 & -2.8747360 & 0.1882490 \\
\hline $\mathrm{H}$ & 6.4918070 & -2.0873130 & 0.1507690 \\
\hline $\mathrm{H}$ & 7.8173290 & 4.7571430 & -0.1918540 \\
\hline $\mathrm{H}$ & 1.5483730 & -3.8905600 & 0.1588420 \\
\hline $\mathrm{H}$ & 3.9208310 & -4.4912780 & 0.2527140 \\
\hline C & 0.4731280 & -1.3821250 & 0.0019600 \\
\hline C & -0.5684240 & -2.2839900 & 0.3749480 \\
\hline C & 0.0261050 & -0.0937760 & -0.4237150 \\
\hline C & -1.8795150 & -1.9384210 & 0.3308470 \\
\hline $\mathrm{H}$ & -0.3397360 & -3.2718650 & 0.7562190 \\
\hline C & -1.2866410 & 0.2436230 & -0.4604220 \\
\hline $\mathrm{H}$ & 0.7296000 & 0.6476950 & -0.7830150 \\
\hline $\mathrm{N}$ & -2.2829410 & -0.6630860 & -0.0817390 \\
\hline $\mathrm{H}$ & -2.6616730 & -2.5935540 & 0.6841450 \\
\hline $\mathrm{H}$ & -1.6302210 & 1.1884200 & -0.8553620 \\
\hline C & -3.6247560 & -0.3082240 & -0.1117760 \\
\hline C & -4.6318650 & -1.2835590 & -0.3259040 \\
\hline C & -4.0222700 & 1.0402500 & 0.0770070 \\
\hline C & -5.9558250 & -0.9341670 & -0.3572640 \\
\hline $\mathrm{H}$ & -4.3599200 & -2.3160050 & -0.5097680 \\
\hline C & -5.3428960 & 1.3980770 & 0.0403820 \\
\hline $\mathrm{H}$ & -3.2810740 & 1.7981200 & 0.3010570 \\
\hline C & -6.3724960 & 0.4260300 & -0.1865490 \\
\hline $\mathrm{H}$ & -6.6843770 & -1.7131820 & -0.5332620 \\
\hline $\mathrm{H}$ & -5.5931840 & 2.4355420 & 0.2161270 \\
\hline$N$ & -7.6764450 & 0.7691260 & -0.2344330 \\
\hline C & -8.6947190 & -0.2909810 & -0.3758130 \\
\hline C & -10.1338220 & 0.1914480 & -0.4522040 \\
\hline $\mathrm{H}$ & -8.5831350 & -0.9773920 & 0.4722960 \\
\hline $\mathrm{H}$ & -8.4628320 & -0.8537810 & -1.2858720 \\
\hline $\mathrm{H}$ & -10.7691100 & -0.6941760 & -0.5252720 \\
\hline $\mathrm{H}$ & -10.4377910 & 0.7449370 & 0.4392940 \\
\hline $\mathrm{H}$ & -10.3161010 & 0.8044230 & -1.3377010 \\
\hline C & -8.0936870 & 2.1503510 & 0.0194650 \\
\hline C & -8.1660900 & 2.4579620 & 1.5155640 \\
\hline $\mathrm{H}$ & -9.0653200 & 2.2983630 & -0.4465310 \\
\hline $\mathrm{H}$ & -7.4043360 & 2.8220410 & -0.4942130 \\
\hline $\mathrm{H}$ & -8.4766740 & 3.4952990 & 1.6617380 \\
\hline $\mathrm{H}$ & -8.8912980 & 1.8055960 & 2.0095300 \\
\hline $\mathrm{H}$ & -7.1938110 & 2.3129180 & 1.9947250 \\
\hline
\end{tabular}


16 (excited-state/keto) G= $-1396.093472 \mathrm{au}$

\begin{tabular}{|c|c|c|c|}
\hline- & -7.1579410 & 0.9223740 & 0.0627040 \\
\hline C & -6.1276060 & 1.8593310 & 0.1384000 \\
\hline C & -6.3432950 & 3.2199950 & 0.2309020 \\
\hline C & -7.6807140 & 3.6153930 & 0.2442630 \\
\hline C & -8.7272050 & 2.6838400 & 0.1683620 \\
\hline C & -8.4916660 & 1.3148650 & 0.0759550 \\
\hline C & -5.1961320 & -0.1187440 & 0.0098460 \\
\hline $\mathrm{H}$ & -9.2978330 & 0.5935670 & 0.0168700 \\
\hline $\mathrm{N}$ & -6.5210960 & -0.3066650 & -0.0155370 \\
\hline $\mathrm{H}$ & -5.5235320 & 3.9255490 & 0.2883680 \\
\hline 0 & -4.9240400 & 1.1918470 & 0.1040160 \\
\hline $\mathrm{H}$ & -9.7512010 & 3.0404260 & 0.1819210 \\
\hline C & -4.1797090 & -1.0985370 & -0.0554780 \\
\hline C & -4.6030890 & -2.4980380 & -0.1642840 \\
\hline C & -2.8283900 & -0.7391610 & -0.0345260 \\
\hline C & -3.5203200 & -3.4682860 & -0.2430440 \\
\hline C & -1.8074820 & -1.7087660 & -0.1108370 \\
\hline $\mathrm{H}$ & -2.5814320 & 0.3155390 & 0.0126650 \\
\hline C & -2.2198840 & -3.0889940 & -0.2204720 \\
\hline 0 & -5.7982100 & -2.8402180 & -0.1868790 \\
\hline $\mathrm{H}$ & -7.9161030 & 4.6714080 & 0.3147100 \\
\hline $\mathrm{H}$ & -1.4597690 & -3.8619750 & -0.2570010 \\
\hline $\mathrm{H}$ & -3.8073280 & -4.5121490 & -0.3107920 \\
\hline C & -0.4160410 & -1.3612350 & -0.0695380 \\
\hline C & 0.6322220 & -2.2665770 & -0.4200270 \\
\hline C & 0.0323520 & -0.0556140 & 0.3173130 \\
\hline C & 1.9411170 & -1.9051020 & -0.3961410 \\
\hline $\mathrm{H}$ & 0.4149790 & -3.2671470 & -0.7729350 \\
\hline C & 1.3466180 & 0.2781840 & 0.3450010 \\
\hline $\mathrm{H}$ & -0.6689690 & 0.6947660 & 0.6609910 \\
\hline $\mathrm{N}$ & 2.3400900 & -0.6238080 & -0.0182280 \\
\hline $\mathrm{H}$ & 2.7259850 & -2.5640320 & -0.7365540 \\
\hline $\mathrm{H}$ & 1.6889840 & 1.2349200 & 0.7124430 \\
\hline C & 3.6860540 & -0.2597380 & -0.0077340 \\
\hline C & 4.6951210 & -1.2180790 & 0.2297450 \\
\hline C & 4.0768450 & 1.0776650 & -0.2353040 \\
\hline C & 6.0221980 & -0.8614670 & 0.2426580 \\
\hline $\mathrm{H}$ & 4.4303820 & -2.2435290 & 0.4618120 \\
\hline C & 5.4020040 & 1.4409710 & -0.2235030 \\
\hline $\mathrm{H}$ & 3.3336580 & 1.8284830 & -0.4794470 \\
\hline C & 6.4314700 & 0.4849330 & 0.0167260 \\
\hline $\mathrm{H}$ & 6.7550340 & -1.6253950 & 0.4686870 \\
\hline $\mathrm{H}$ & 5.6519660 & 2.4710790 & -0.4428820 \\
\hline$N$ & 7.7394070 & 0.8395680 & 0.0287600 \\
\hline C & 8.8067120 & -0.1504140 & 0.1551830 \\
\hline C & 9.1317520 & -0.4709070 & 1.6129700 \\
\hline $\mathrm{H}$ & 9.6865580 & 0.2636220 & -0.3452040 \\
\hline $\mathrm{H}$ & 8.5307650 & -1.0506220 & -0.3991390 \\
\hline $\mathrm{H}$ & 9.9424600 & -1.2022360 & 1.6650380 \\
\hline $\mathrm{H}$ & 9.4478430 & 0.4326410 & 2.1420730 \\
\hline $\mathrm{H}$ & 8.2589880 & -0.8804520 & 2.1293780 \\
\hline C & 8.1636880 & 2.2333070 & -0.0841630 \\
\hline C & 8.3095090 & 2.6802800 & -1.5375740 \\
\hline $\mathrm{H}$ & 9.1229090 & 2.3175340 & 0.4344770 \\
\hline $\mathrm{H}$ & 7.4610540 & 2.8691470 & 0.4596220 \\
\hline $\mathrm{H}$ & 8.6443280 & 3.7199740 & -1.5788930 \\
\hline $\mathrm{H}$ & 9.0456730 & 2.0601380 & -2.0570010 \\
\hline $\mathrm{H}$ & 7.3581650 & 2.5992140 & -2.0708890 \\
\hline
\end{tabular}


16 (excited-state/ESIPT TS) G= -1396.090444au, $v(\mathrm{imag})=1091 i \mathrm{~cm}^{-1}$

\begin{tabular}{|c|c|c|c|}
\hline 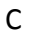 & -7.2049940 & 0.7927290 & 0.0446630 \\
\hline C & -6.2777110 & 1.8329800 & 0.1368430 \\
\hline C & -6.6296300 & 3.1661020 & 0.2274810 \\
\hline C & -7.9989060 & 3.4244430 & 0.2215000 \\
\hline C & -8.9460230 & 2.3918440 & 0.1293420 \\
\hline C & -8.5717320 & 1.0551700 & 0.0393140 \\
\hline C & -5.1845470 & -0.0326620 & 0.0178650 \\
\hline $\mathrm{H}$ & -9.3006820 & 0.2567570 & -0.0320190 \\
\hline $\mathrm{N}$ & -6.4571110 & -0.3756820 & -0.0275940 \\
\hline $\mathrm{H}$ & -5.8867140 & 3.9513560 & 0.2974190 \\
\hline 0 & -5.0095820 & 1.2899080 & 0.1188570 \\
\hline $\mathrm{H}$ & -10.0006500 & 2.6445730 & 0.1283880 \\
\hline C & -4.1305970 & -0.9941270 & -0.0424540 \\
\hline C & -4.5669370 & -2.3680230 & -0.1651090 \\
\hline C & -2.7828440 & -0.6460320 & -0.0071510 \\
\hline C & -3.5268200 & -3.3498770 & -0.2447420 \\
\hline C & -1.7751260 & -1.6255490 & -0.0884400 \\
\hline $\mathrm{H}$ & -2.5292380 & 0.4070830 & 0.0545320 \\
\hline C & -2.2113320 & -2.9893070 & -0.2099480 \\
\hline 0 & -5.8049190 & -2.6921020 & -0.2009610 \\
\hline $\mathrm{H}$ & -6.4985100 & -1.5413100 & -0.1191120 \\
\hline $\mathrm{H}$ & -8.3408190 & 4.4512390 & 0.2895280 \\
\hline $\mathrm{H}$ & -1.4675060 & -3.7780460 & -0.2489180 \\
\hline $\mathrm{H}$ & -3.8252690 & -4.3894700 & -0.3250590 \\
\hline C & -0.3776350 & -1.2890910 & -0.0481850 \\
\hline C & 0.6570770 & -2.2022210 & -0.4210290 \\
\hline C & 0.0894310 & 0.0010100 & 0.3629250 \\
\hline C & 1.9703130 & -1.8639800 & -0.3962180 \\
\hline $\mathrm{H}$ & 0.4212490 & -3.1927310 & -0.7905940 \\
\hline C & 1.4049960 & 0.3240530 & 0.3856340 \\
\hline $\mathrm{H}$ & -0.6015830 & 0.7521680 & 0.7261400 \\
\hline $\mathrm{N}$ & 2.3912420 & -0.5888850 & -0.0000820 \\
\hline $\mathrm{H}$ & 2.7420990 & -2.5281170 & -0.7547710 \\
\hline $\mathrm{H}$ & 1.7592240 & 1.2677980 & 0.7732340 \\
\hline C & 3.7342260 & -0.2421110 & 0.0045330 \\
\hline C & 4.7408570 & -1.2240360 & 0.1817450 \\
\hline C & 4.1380280 & 1.1054110 & -0.1687650 \\
\hline C & 6.0686440 & -0.8830710 & 0.1850960 \\
\hline $\mathrm{H}$ & 4.4684800 & -2.2552410 & 0.3730140 \\
\hline C & 5.4637790 & 1.4534870 & -0.1618490 \\
\hline $\mathrm{H}$ & 3.3993950 & 1.8734980 & -0.3654650 \\
\hline C & 6.4884220 & 0.4725230 & 0.0142020 \\
\hline $\mathrm{H}$ & 6.7982320 & -1.6629070 & 0.3609260 \\
\hline $\mathrm{H}$ & 5.7229980 & 2.4898210 & -0.3353840 \\
\hline $\mathrm{N}$ & 7.7949960 & 0.8114630 & 0.0179770 \\
\hline C & 8.8577440 & -0.1926540 & 0.0795900 \\
\hline C & 9.2080530 & -0.5702570 & 1.5179020 \\
\hline $\mathrm{H}$ & 9.7285530 & 0.2380820 & -0.4217420 \\
\hline $\mathrm{H}$ & 8.5609920 & -1.0661840 & -0.5044940 \\
\hline $\mathrm{H}$ & 10.0174070 & -1.3044650 & 1.5218790 \\
\hline $\mathrm{H}$ & 9.5378150 & 0.3101030 & 2.0765270 \\
\hline $\mathrm{H}$ & 8.3444000 & -0.9992910 & 2.0334290 \\
\hline C & 8.2361050 & 2.2055960 & -0.0390690 \\
\hline C & 8.3625420 & 2.7101440 & -1.4755390 \\
\hline $\mathrm{H}$ & 9.2052860 & 2.2506520 & 0.4647330 \\
\hline $\mathrm{H}$ & 7.5509750 & 2.8237900 & 0.5446600 \\
\hline $\mathrm{H}$ & 8.7123420 & 3.7454190 & -1.4752500 \\
\hline
\end{tabular}


$\begin{array}{llll}\text { H } & 9.0806450 & 2.1032920 & -2.0339160\end{array}$

$\begin{array}{llll}\text { H } & 7.4007040 & 2.6664660 & -1.9937820\end{array}$ 


\section{S5 References}

(1) Y. Zhao, D. G. Truhlar, The M06 suite of density functionals for main group thermochemistry, thermochemical kinetics, noncovalent interactions, excited states, and transition elements: two new functionals and systematic testing of four M06-class functionals and 12 other functionals, Theor. Chem. Acc. 2008, 120, 215- 241.

(2) Gaussian 16, Revision A.03, M. J. Frisch, et al., Gaussian, Inc., Wallingford CT, 2016.

(3) R. Cammi, B. Mennucci, Linear response theory for the polarizable continuum model, J. Chem. Phys. 1999, 110, 9877-9886

(4) C. Azarias, S. Budzak, A. D. Laurent, G. Ulrich, D. Jacquemin, Tuning ESIPT fluorophores into dual emitters, Chem. Sci. 2016, 7, 3763-3774.

(5) P. M. Vérité, C. A. Guido and D. Jacquemin, First-principles investigation of the double ESIPT process in a thiophene-based dye, Phys. Chem. Chem. Phys., 2019, 21, 2307-2317.

(6) O. Christiansen, H. Koch and P. Jørgensen, The second-order approximate coupled cluster singles and doubles model CC2, Chem. Phys. Lett., 1995, 243, 409-418.

(7) TURBOMOLE V7.3 2016, a development of University of Karlsruhe and Forschungszentrum Karlsruhe GmbH, 1989 - 2007, TURBOMOLE GmbH, since 2007; available from http://www.turbomole.com 